\title{
IRON AND PROCHLOROCOCCUS
}

By

Anne Williford Thompson

B.A., Middlebury College, 2002

Submitted in partial fulfillment of the requirements for the degree of

Doctor of Philosophy

at the

MASSACHUSETTS INSTITUTE OF TECHNOLOGY

and the

WOODS HOLE OCEANOGRAPHIC INSTITUTION

June 2009

(C) 2009 Anne Williford Thompson

All rights reserved.

The author hereby grants to MIT and WHOI the permission to reproduce and to distribute publicly paper and electronic copies of this thesis document in whole or in part in any medium now known or hereafter created.

Signature of Author

Certified by

Joint Program in Biological Oceanography Massachusetts Institute of Technology and Woods Hole Oceanographic Institution April $7^{\text {th }}, 2009$

\begin{tabular}{rr}
\hline $\begin{array}{r}\text { Sallie W. Chisholm } \\
\text { Thesis Supervisor }\end{array}$ \\
\hline $\begin{array}{r}\text { Mak A. Saito } \\
\text { Thesis Supervisor }\end{array}$
\end{tabular}

Accepted by

Simon Thorrold Chair, Joint Committee for Biological Oceanography Woods Hole Oceanographic Institution 



\title{
IRON AND PROCHLOROCOCCUS
}

\author{
by \\ Anne Williford Thompson \\ Submitted in partial fulfillment of the requirement \\ for the degree of Doctor of Philosophy in \\ Biological Oceanography
}

\begin{abstract}
Iron availability and primary productivity in the oceans are intricately linked through photosynthesis. At the global scale we understand how iron addition induces phytoplankton blooms through meso-scale iron-addition experiments. At the atomic scale, we can describe the length and type of bonds that connect iron atoms to components of photosystem I, the most efficient light-harvesting complex in nature. Yet, we know little of how iron influences microbial diversity and distribution in the open ocean. In this study, we assess the influence of iron on the ecology of the numerically abundant marine cyanobacterium, Prochlorococcus. With its minimal genome and ubiquity in the global ocean, Prochlorococcus represents a model system in which to study the dynamics of the link between iron and primary productivity. To this end, we tested the iron physiology of two closely-related Prochlorococcus ecotypes. MED4 is adapted to high-light environments while MIT9313 lives best in low-light conditions. We determined that MIT9313 is capable of surviving at low iron concentrations that completely inhibit MED4. Furthermore, concentrations of Fe' that inhibit growth in culture are sufficient to support Prochlorococcus growth in the field, which raises questions about the species of iron available to Prochlorococcus. We then examined the molecular basis for the ability of MIT9313 to grow at lower iron concentrations than MED4 by assessing whole-genome transcription in response to changes in iron availability in the two ecotypes. Genes that were differentially expressed fell into two categories: those that are shared by all (Prochlorococcus core genome) and those that are not (non-core genome). Only three genes shared between MED4 and MIT9313 were iron-responsive in both strains. We then tested the iron physiology of picocyanobacteria in the field and found that Synechococcus is iron-stressed in samples where Prochlorococcus is not. Finally, we propose a method to measure how iron stress in Prochlorococcus changes over natural gradients of iron in the oligotrophic ocean by quantifying transcription of the iron-stress induced gene, isiB. Taken together, our studies demonstrate that iron metabolism influences the ecology of Prochlorococcus both by contributing to its diversity and distinguishing it from other marine cyanobacteria.
\end{abstract}

Thesis Supervisors: Sallie W. Chisholm ${ }^{1}$ and Mak A. Saito ${ }^{2}$

Titles: ${ }^{1}$ Professor of Civil and Environmental Engineering, and Biology at the Massachusetts Institute of Technology

${ }^{2}$ Associate Scientist in Marine Chemistry \& Geochemistry at the Woods Hole Oceanographic Institution 



\section{ACKNOWLEDGEMENTS}

I am grateful for the opportunity to formally express my thanks to so many people for their guidance and support throughout the years I spent in the MIT-WHOI Joint Program. First of all, I thank Penny Chisholm for the education in science that she has bestowed upon me as her student. Penny is an expert at thinking about the big picture and sets high standards for the quality of work that comes out of her lab. Penny is not only skilled in science but talented at seeing those in her lab as whole people and caring for our wellbeing in many ways. Penny's emotional support was essential to keep me moving forward at many points during this process and I am tremendously grateful to her for her encouragement of my interests outside of the lab.

Next, I am proud to thank my co-advisor, Mak Saito, for his guidance in the lab and expertise on trace-metal chemistry. Mak's example of how to carryout trace-metal clean experiments at sea and in the lab was essential. Mak was also always willing to spend time with me discussing key concepts in trace-metal chemistry and how to interpret my results. In addition, while directing an ambitious and complex sampling plan aboard the R/V Knorr, Mak always exhibited patience and positive energy. This is a leadership style to which I aspire.

I am grateful to my committee member James Moffett for sample collection, his expertise on trace metals in the ocean imparted during class and during discussions, and for including me in his research cruise off the coast of Peru in 2005. I am also grateful to Eric Webb both as a committee member and as a professor in several classes concerning biological oceanography and the use of iron by marine microbes. I thank John Waterbury, the chair of my defense, for his time and the use of incubator space at WHOI.

The Chisholm Lab is an environment rich with intellect, research experience, and friendship and I am fortunate to have been a part of this group. In particular, Maureen Coleman and Katherine Huang have been incredibly helpful in teaching me how to carryout comparative genomic analysis, process microarray data, and understand environmental sequence data in the context of my project. Jake Waldbauer and Rex Malmstrom were indispensable in their instruction in flow cytometry and willingness to troubleshoot the flow cytometer when I needed help. I also appreciate those who I have worked closely with or who have provided essential advice including Claudia Steglich, Debbie Lindell, Liz Mann, Matt Sullivan, Adam Martiny, Lisa Moore, Sébastien Rodrigue, Daniel Sher, Libusha Kelly, Paul Berube, and Laura Croal. I specially thank Allison Coe, Deborah Fullerton, and Marcia Osburne for their role in supporting the work of and creating community within the Chisholm Lab.

All members of the Saito Lab are seasoned oceanographers and I am grateful to have spent time with these scientists at sea and in the trace-metal clean lab at WHOI. I am particularly obliged to Tyler Goepfert for his hard work at sea as well as his ability think and work creatively to make sampling run smoothly. Thanks to Erin Bertrand for helping me monitor the growth of cultures.

My friends have insured that I have learned more than science in the past 6 years. I thank Becca and Chris Neumann for their patience in teaching me to ski and kayak. Stephanie Waterman, Ari Shapiro, and Kjetil and Selina Våge have provided the support of a family at our apartment in Somerville. I especially thank Arne Bomblies for the balance and diversion he has added to my life.

My family has been a constant source of comfort, joy, and adventure in my graduate years. I especially thank my parents, Alice and Todd, for all they have done.

This work was supported by grants from the Gordon and Betty Moore Foundation (\#495 and \#495.01), the National Science Foundation (OCE-0425602), the Center for Microbial 
Oceanography: Research and Education established by the National Science Foundation (Z792093-02), and the Department of Energy (DE-FG02-07ER64506 and DE-FG02-08ER64516) to Sallie W. Chisholm. In addition, the work was supported by grants to Mak A. Saito from the Office of Naval Research (N00014-05-1-0704), National Science Foundation Center for Microbial Oceanography: Research and Education (Z792384), National Science Foundation Environmental Genomics Program (0723667), and National Science Foundation Chemical Oceanography Program (OCE-0752271 and OCE-0452883). 


\section{TABLE OF CONTENTS}

PAGE

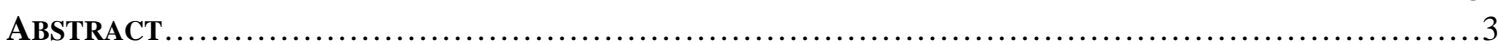

ACKNOWLEDGEMENTS......................................................................

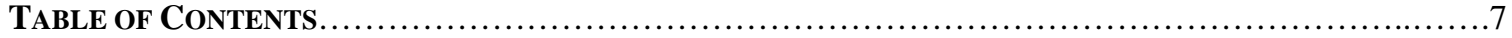

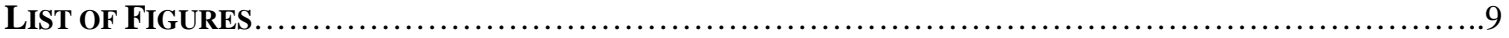

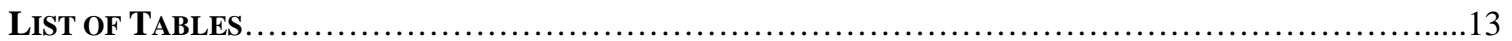

CHAPTER 1: Introduction.................................................................... 15

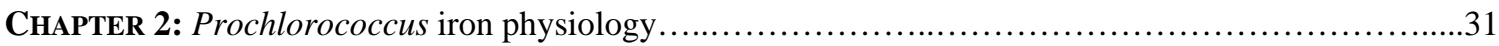

CHAPTER 3: Prochlorococcus whole-genome response to changes in iron availability..................65

CHAPTER 4: Prochlorococcus iron physiology in the Eastern Equatorial Pacific and diversity

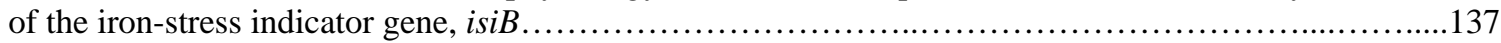

CHAPTER 5: Conclusions and Future Directions.............................................161

APPENDIX A: Use of stable isotope-labeled cells to identify active grazers of picocyanobacteria in ocean surface waters.

Frias-Lopez et al. (2009) EM 11: 512-525.....

APPENDIX B: Updated analysis and comparisons of Prochlorococcus whole-genome expression in response to iron starvation, nitrogen starvation, phosphorus starvation, light stress, and phage infection

APPENDIX C: Reliability of expired custom M4-9313 Affymetrix microarray chips 220

APPENDIX D: The effect of centrifugation on Prochlorococcus whole-genome expression.

APPENDIX E: Phylogeny of Prochlorococcus in an oxygen minimum zone off the coast of Mexico.

APPENDIX F: Scanning electron microscopy of a non-axenic culture of Prochlorococcus, MIT9215

APPENDIX G: Abundance and distribution of Prochlorococcus and Synechococcus in the Eastern Equatorial Pacific Ocean.

APPENDIX H: The Prochlorococcus and Synechococcus response to iron and cobalt enrichment in the Costa Rica Upwelling Dome in July 2005. 



\section{Chapter 1}

\section{LIST OF FIGURES}

Figure 1: Iron-related genes identified in the Prochlorococcus core genome....

\section{Chapter 2}

Figure 1: Steady-state growth rates over a range of iron concentrations and at two light intensities........40

Figure 2: Red fluorescence per cell measured by flow cytometry relative to standard beads over a range of iron concentrations and at two light levels....................................... 41

Figure 3: Forward scatter measured by flow cytometry relative to standard beads over a range of iron

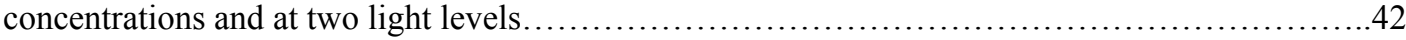

Figure 4: MED4ax compared to MIT9313ax over a range of iron concentrations......................44

Figure 5: Study in the effect of light availability on the concentration of Fe' that induces iron stress in

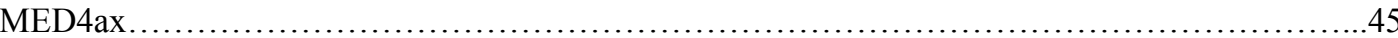

Figure 6: Comparison of iron concentrations at IronExII that support half-maximum growth rates in natural Prochlorococcus populations compared to Prochlorococcus growth rates in culture........48

Figure 7: Carbon per cell in MED4ax compared to relative forward scatter as a proxy for cell size over a range of iron concentrations and at two light levels

Figure 8: Dissolved organic carbon (DOC) concentrations measured in MED4ax and MIT9313ax over a range of iron concentrations and at $20 \mu \mathrm{E}^{-2} \mathrm{~s}^{-1}$

Figure 9: Intracellular metal concentrations of MED4ax and MIT9313ax at 0.19nM Fe' and at two light

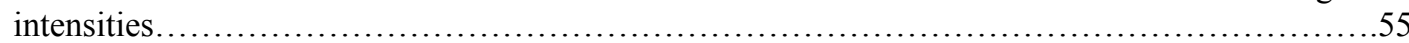

Figure 10: Intracellular metal concentrations of MED4ax and MIT9313ax at 0.19nM Fe' and at two light

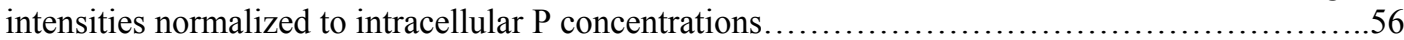

Figure 11: Intracellular trace metal ratios of Fe, Co, and Mn in MED4ax and MIT9313ax at 0.19nM Fe' and a two different light intensities.................................................57

\section{Chapter 3}

Figure 1: Growth of Prochlorococcus MED4 and MIT9313 during iron stress and recovery..............76

Figure 2: Changes in mean relative red fluorescence per cell and mean relative forward scatter per cell in individual MED4 and MIT9313 cells during iron stress and recovery...................77

Figure 3: MED4 photosynthetic efficiency and isiB expression during iron stress. .....................79

Figure 4: Global expression response of Prochlorococcus MED4 and MIT9313 to iron deprivation and recovery from iron starvation............................................ 81

Figure 5: Mean expression profiles of clusters of differentially expressed genes.........................82

Figure 6: Expression of MED4-MIT9313 orthologs and non-orthologs to iron rescue...................84

Figure 7: Expression profile of iron-responsive MED4-MIT9313 orthologs .........................85

Figure 8: MED4 diel expression of Prochlorococcus core genes that are predicted to be related to iron.....88

Figure 9: Distribution of all MED4 or MIT9313 iron-responsive genes among the genomes of other

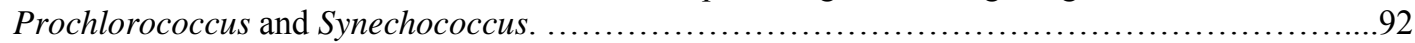

Figure 10: Genome position of iron-responsive genes in MED4 ......................................94

Figure 11: Genome position of iron-responsive genes in MIT9313 ...............................95

Figure 12: idiA/afuA genome region among sequenced Prochlorococcus isolates......................96

Figure 13: Response of Prochlorococcus MED4 and MIT9313 photosynthesis genes to iron

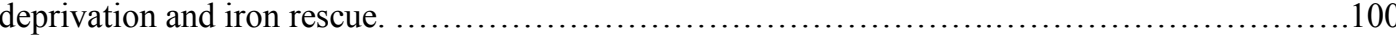

Figure 14: Response of Prochlorococcus MED4 and MIT9313 high-light inducible protein genes (hlips) to iron deprivation and iron rescue.

Figure 15: Response of Prochlorococcus MED4 and MIT9313 small non-coding RNAs to iron deprivation and iron rescue.

Figure 16: Comparison of the global expression response in Prochlorococcus MED4 to iron starvation at 24 hours (x-axis) and all time-points of phosphorous starvation (y-axis) . ............107

Figure 17: Comparison of the global expression response in Prochlorococcus MIT9313 to iron starvation at 24 hours (x-axis) and all time-points of phosphorous starvation (y-axis).

Figure 18: Comparison of the global expression response in Prochlorococcus MED4 to iron 
starvation at 24 hours (x-axis) and all time-points of phage infection (y-axis).

Figure 19: Comparison of the global expression response in Prochlorococcus MED4 to iron starvation at 24 hours (x-axis) and all time-points of nitrogen starvation (y-axis).

Figure 20: Comparison of the global expression response in Prochlorococcus MIT9313 to iron starvation at 28 hours (x-axis) and all time-points of nitrogen starvation (y-axis) . ...............114

Figure 21: Comparison of the global expression response in Prochlorococcus MED4 to iron starvation at 24 hours (x-axis) and shifts in light availability (y-axis).

\section{Chapter 4}

Figure 1: Station locations of bottle incubation experiments conducted on the KN182-5 cruise in July/August 2005 and the KN182-9 cruise conducted in October/November 2005. .............142

Figure 2: Alignments of isiB sequences for primer design.................................... 145

Figure 3: Single time-point bottle incubation experiments conducted at Station 17 in August 2005 during the KN182-5 cruise.

Figure 4: Time-course bottle incubation experiments conducted at Station 14 in October 2005 during the KN182-9 cruise.

Figure 5: Neighbor-joining tree showing the diversity among Prochlorococcus and Synechococcus flavodoxin (isiB) sequences from environmental databases.

\section{Appendix A}

Figure 1: Phylogenetic assignments and relative frequencies of the rDNA sequences from indigenous eukaryotic community, and the labelled and unlabelled rRNA fractions in the experimental treatments.

Figure 2: Unrooted phylogenetic tree inferred by maximum likelihood (ML) analysis of the reference sequences used in the phylogenetic analysis of the clone libraries presented in this work..........171

Figure 3: Relative amount of rRNA in different fractions separated by density gradient centrifugation of $18 \mathrm{~S}$ rRNA analysed in this study.

Figure 4: Self-organizing tree (SOTA) of terminal restriction fragment length polymorphism (T-RFLP) profiles emerging from the $18 \mathrm{~S}$ rRNA sequences from the different experimental treatments.....172

Figure S1: Phylogenetic analysis of the sequences derived from the $18 \mathrm{~S}$ rDNA sequences in the

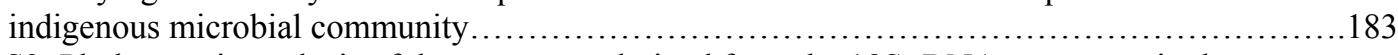

Figure S2: Phylogenetic analysis of the sequences derived from the $18 \mathrm{~S}$ rDNA sequences in the

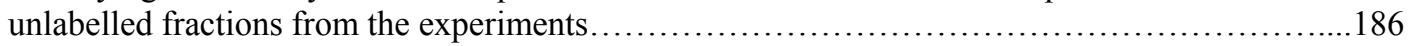

Figure S3: Phylogenetic analysis of the sequences derived from the labelled 18S rDNA sequences from the experimental bottles amended with labelled Prochlorococcus cells.....................189

Figure S4: Phylogenetic analysis of the sequences derived from the labelled 18S rDNA sequences from the experimental bottles amended with labelled Synechococcus cells.......................192

Figure S5: Phylogenetic tree 16S rRNA sequences from chloroplasts and bacteria inferred by maximum likelihood (ML) analysis.

\section{Appendix B}

Figure 1-8: Significance thresholds used to identify differentially expressed genes in MED4 and MIT9313 in response to nitrogen, phosphorus, phage infection, light, and iron.

Figure 9-12: Percents of MED4 or MIT9313 differentially-expressed genes that are present in other

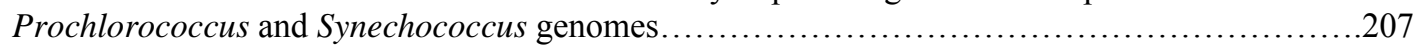

Figure 13-14: Genome position of iron-, nitrogen-, and phosphorus-responsive genes in MED4 and

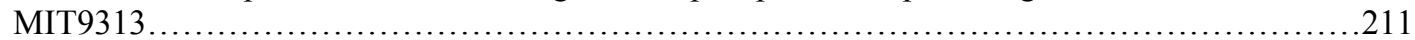

Figure 15: Genome position of iron-, nitrogen-, phosphorus-, light-, and phage-responsive genes in MED4

Figure 16-18: Diel expression patterns and cluster membership of iron-responsive genes that cluster with isiB, afuA, and petF.

Figure 19: Diel expression of genes predicted to be involved in iron metabolism 


\section{Appendix C}

Figure 1: Reliability of expired M4-9313 Affymetrix Arrays

\section{Appendix D}

Figure 1: The effect of centrifugation on MED4 whole-genome expression.

\section{Appendix E}

Figure 1: Phylogeny of Prochlorococcus from an oxygen minimum zone (OMZ) off the coast of Mexico in 2003.

\section{Appendix F}

Figure 1: Scanning electron microscopy images of non-axenic MIT9215.

\section{Appendix G}

Figure 1: KN182-5 and KN182-9 cruise tracks.

Figure 2: Prochlorococcus and Synechococcus abundances in surface transect through the Eastern Equatorial Pacific on cruise KN182-9.

Figure 3: Abundances of Prochlorococcus and Synechococcus in the Peru Upwelling

Region on cruise KN182-9.

Figure 4: Abundances of Prochlorococcus and Synechococcus in the vicinity of the Costa

Rica Upwelling Dome on cruise KN182-5.

Figure 5: Surface abundances of Prochlorococcus and Synechococcus across the Costa

Rica Upwelling Dome on cruise KN182-5.

\section{Appendix H}

Figures 1-3: Bottle incubation experiments on cruise KN182-5. 



\section{LIST OF TABLES}

Chapter 2

PAGE

Table 1: Iron-replete PRO99-TM media composition

\section{Chapter 3}

Table 1: Primers used for quantitative reverse-transcriptase PCR analysis of $r n p B$ and isiB .............73

Table 2: MED4 genes that are differentially expressed in response to change in iron availability ........122

Table 3: MIT9313 genes that are differentially expressed in response to changes in iron availability......127

\section{Chapter 4}

Table 1: Best candidates for flavodoxin (isiB) PCR primers.

\section{Appendix A}

Table S1: Oligonucleotides used for the amplification of 16S rRNA chloroplast genes from different groups defined based on the ARB tree

\section{Appendix B}

Table 1: Normalization and re-analysis of published experiments with the Affymetrix array M4-9313...198 



\section{CHAPTER ONE}

Introduction 


\section{INTRODUCTION}

Iron plays a critical role in primary productivity in the ocean. At the global scale, phytoplankton growth in response to iron addition to natural seawater demonstrates the importance of iron in fueling primary productivity (Boyd et al, 2007; Martin et al, 1990a; Martin et al, 1990b). At the molecular scale, $2.5 \AA$ resolution of the crystal structure of the photosystem I complex shows how 12 atoms of iron are positioned to facilitate electron transfer in nature's most efficient light-harvesting complex (Jordan et al, 2001). Yet, between the angstrom scale and the kilometer scale, how we understand the connection between iron biogeochemical cycles and the global carbon cycle is complicated and unclear. We know little about which forms of iron are available to phytoplankton, how phytoplankton acquire iron from the environment, and how much iron is required to sustain growth in the oligotrophic open oceans. As an abundant oxygenic phototroph with a minimal genome, Prochlorococcus represents a simple link between iron and primary productivity in the marine environment. In this body of work, we aim to describe how the ecology of Prochlorococcus is influenced by iron.

\section{Iron in the ocean}

Until the proliferation of oxygenic phototrophs and concomitant increase in the concentration of oxygen in the atmosphere, iron is thought to have existed in the soluble ferrous state in marine environments (Dauphas et al, 2004; Hunter and Boyd, 2007; Kump, 2005). When oxygen concentrations became higher, iron chemistry in the marine environment shifted to favor the prevalence of ferric iron, which readily associates with oxygen as ferric hydroxides and is insoluble at neutral $\mathrm{pH}$. Despite this shift in iron speciation in the ocean over geologic time, the ease of redox reactions between the ferric and ferrous forms of iron under physiological conditions, and its ability to assume different spin states in both redox forms, make iron an extremely valuable element for life.

Today, we know that iron concentration and speciation varies greatly along the horizontal, vertical, and temporal dimensions of the euphotic zone of the open ocean where Prochlorococcus is abundant. Patterns of iron concentration along the horizontal dimension are greatly influenced by the sources of iron to the marine environment, which include riverine input, hydrothermal upwelling, and atmospheric deposition (Jickells et al, 2005). While the amount of iron introduced to the oceans from riverine and hydrothermal sources is high, precipitation occurs 
rapidly in both cases and removes iron from seawater, minimizing the impact of hydrothermal and riverine sources on the concentration of iron in the surface waters of large ocean gyres (Bruland et al, 2003; Jickells et al, 2005). Atmospheric iron inputs, on the other hand, are a direct and major source of iron to the open ocean euphotic zone (Bruland et al, 2003; Duce and Tindale, 1991). Through recognizing atmospheric input as a dominant control on surface water iron concentrations, numerical models can roughly estimate global patterns of atmospheric iron deposition using field measurements and satellite data on the distribution of atmospheric aerosols (Bruland et al, 2003; Duce et al, 1991; Jickells et al, 2005; Mahowald et al, 1999 ; Moore et al, 2004; Tegen and Fung, 1995 ). Both observations and model predictions show very low concentrations of iron in the remote open-ocean and high-nutrient low-chlorophyll regions (HNLC). Numerical models also suggest that biological processes may be important in controlling iron concentrations in surface waters (Bruland et al, 2003; Jickells et al, 2005). As the numerically dominant open-ocean phytoplankton group, we expect Prochlorococcus to play an important role in controlling iron concentrations and speciation through its activity as a primary producer.

The influence of biological activity on controlling iron distribution is reflected in the profile of iron concentration over depth. Iron behaves both as a nutrient-type element and as a scavenged element in the ocean, and has been called a "hybrid" element (Bruland et al, 2003). In remote surface waters like HNLC regions, iron exhibits a nutrient-type element profile with depletion in the surface waters and regeneration at depth (Bruland et al, 2003; Johnson et al, 1997; Martin and Gordon, 1988). The scavenged-element aspect of iron distribution is apparent in oligotrophic central gyres and areas of high dust input where iron displays a surface water maximum much like aluminum, a typical scavenged element (Bruland et al, 2003). Interestingly, it appears that the different size classes of iron display either aspect of iron's hybrid-element behavior. The vertical profile of soluble iron $(<0.02 \mu \mathrm{m})$ resembles a nutrient-type profile similar to that of major nutrients phosphate and nitrogen and the dissolved pool of iron $(<0.4 \mu \mathrm{m})$. Colloidal iron $(0.02 \mu \mathrm{m}$ to $0.4 \mu \mathrm{m})$, displays a deviant trend, showing a surface maximum and a minimum in the upper nutricline (Wu et al, 2001). Iron enrichment experiments in the Southern Ocean, coastal waters of Canada, and eastern Bering Sea demonstrate that colloidal iron is takenup more quickly than particulate and soluble fractions during phytoplankton blooms and that the concentration of colloidal iron appears to be more dynamic than soluble or dissolved iron (Hurst and Bruland, 2007; Nishioka et al, 2005; Nishioka et al, 2001). These results are intriguing, yet 


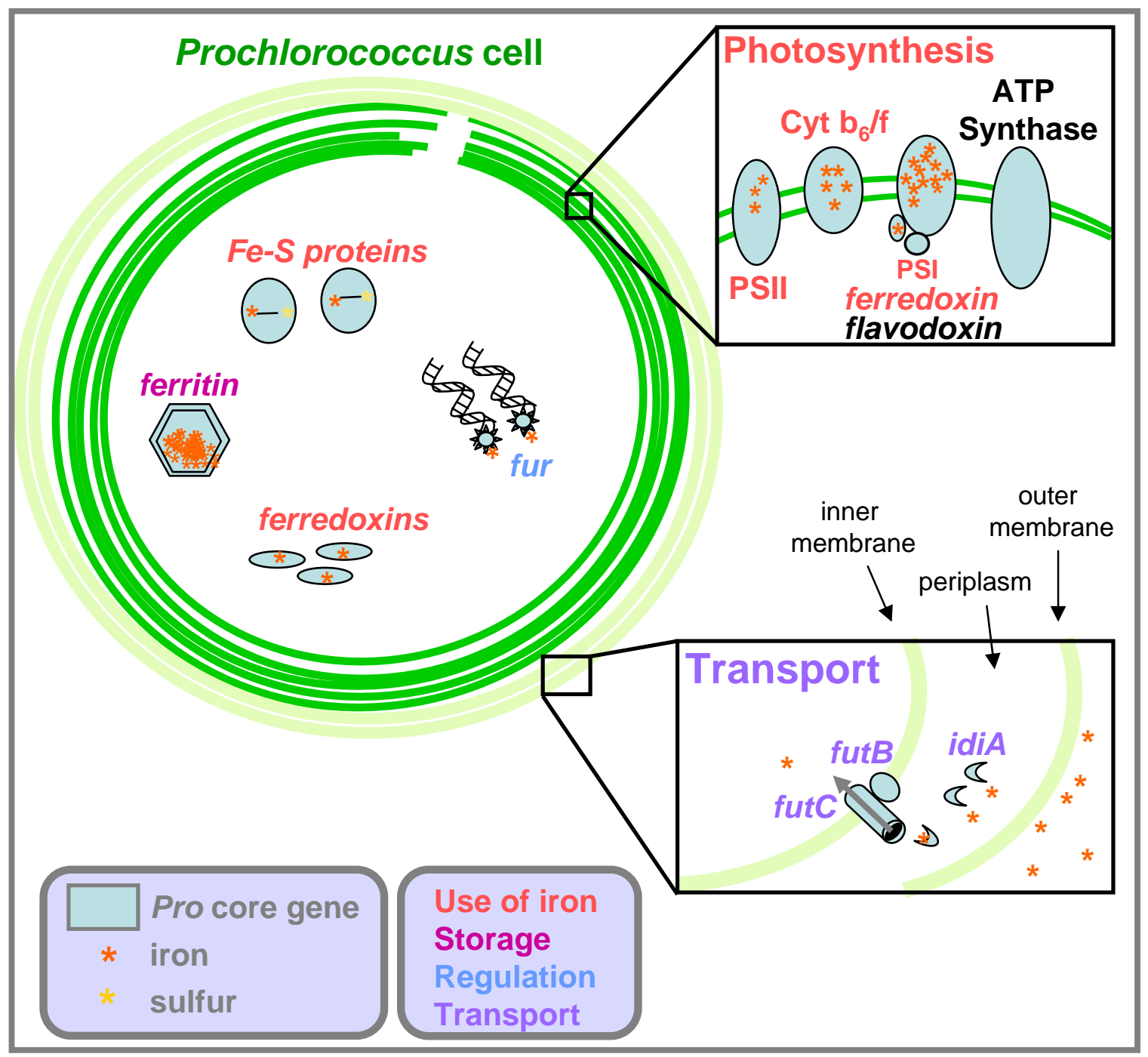

Figure 1: Iron-related genes identified in the Prochlorococcus core genome. Light-green lines represent the inner and outer membranes of the cell. Dark-green lines represent the thylakoid membranes where photosynthesis takes place. Iron is involved in photosynthesis (upper-right box) where it is associated with the photosystem I (PSI) and photosystem II (PSII) complexes, the cytochrome $b_{6} / f$ complex $\left(C_{1} b_{6} / f\right)$, as well as ferredoxin. Flavodoxin is an iron-free replacement to ferredoxin and is also present in the core genome. Several genes encoding Fe-S proteins and ferredoxins are also present in the Prochlorococcus core genome and likely carry-out a range of functions. The ferric uptake regulator (fur) is present in two copies in the core genome. Additionally, ferritin, an iron-storage protein is present in the Prochlorococcus core genome. Three genes are homologous to known iron transport genes include futB, fut $C$ and idiA. The role of idiA in Prochlorococcus is uncertain as in some cyanobacteria it encodes a protein associated with the thylakoid membrane and in others encodes a protein located in the periplasm (see text). 
the bioavailability of the colloidal fraction compared to the soluble fraction of dissolved iron remains an open question.

Researchers agree that most Fe(III) is complexed to strong organic ligands in surface waters, which likely contribute to maintaining a pool of dissolved iron in ocean waters (Hunter et al, 2007; Rue and Bruland, 1995; van den Berg, 1995). $\mathrm{L}_{1}$ and $\mathrm{L}_{2}$ are two major ligand classes that are operationally defined and appear to be present in both the colloidal and soluble size fractions with maximum concentrations in the mixed layer (Hunter et al, 2007). Two studies show that Fe-binding ligands in the soluble size class are depleted at the surface, yet otherwise the vertical and horizontal distributions of iron-binding organic ligands are unclear (Boye et al, 2006; Wu et al, 2001).

The susceptibility of iron-ligand complexes to photochemical reactions gives light an important influence on iron speciation and concentration in the euphotic zone and adds a third dimension, time, to how we understand iron in the ocean. Photo-reduction of Fe(III) bound to an organic ligand can occur either through ligand to metal charge transfer reactions (LMCT) following direct photolysis by the absorption of light, or, through reactions with radical species that are produced photochemically (Barbeau, 2006). A modeling study shows how light availability influences the concentration of $\mathrm{FeL}, \mathrm{Fe}^{2+}, \mathrm{Fe}^{3+}$ concentrations in the surface ocean over a 24-hour period (Weber et al, 2007). It will be interesting follow future work on how photochemical reactions influence iron speciation as these effects are likely to significantly affect iron availability to the microbial community.

Thus, we see that iron speciation and distribution in the habitat of Prochlorococcus is controlled by diverse and complex processes. Johnson et al. 1997 suggests that the complex nature of iron distribution in the ocean (as compared to the distribution of other elements) points to the activity of unique processes in controlling its distribution. We expect that many of these processes are related to the activity of the microbial community. As a numerically dominant phytoplankton, we expect the activity of Prochlorococcus to contribute significantly to the unique processes that control marine iron biogeochemical cycles.

\section{Prochlorococcus - what we know}

Prochlorococcus is the numerically dominant oxygenic phototroph in the tropical and sub-tropical open oceans (Partensky et al, 1999). The different ecotypes of Prochlorococcus are specially adapted for gradients in nutrient availability and physical conditions along the spatial 
and temporal dimensions of the open ocean (Coleman and Chisholm, 2007). The diversity of Prochlorococcus ecotypes is obvious at the genomic level, physiological level, and in its global distributions. For example, comparative genomics shows that the extent of the diversity among Prochlorococcus genomes has not been reached as the total number of unique genes increases with each additional sequenced strain (non-core genome) (Kettler et al, 2007). The identification of genomic islands in the Prochlorococcus genomes suggests the importance of adaptation to local environmental conditions and diversification via horizontal gene transfer (Coleman et al, 2006). Ecotypes differ greatly in their light and nitrogen physiologies as well as their susceptibility to phage infection and copper toxicity (Mann and Chisholm, 2000; Moore and Chisholm, 1999; Moore et al, 2002; Sullivan et al, 2003). Furthermore, high-light adapted ecotypes are most abundant at the surface while low-light adapted ecotypes thrive at depth (Zinser et al, 2006) and the distribution of ecotypes over open-ocean transects corresponds to their nitrogen and temperature physiologies (Johnson et al, 2006). Prochlorococcus ecotypes are similar in many respects, as well. Most notably, all twelve sequenced genomes share about 1200 genes that make up the core genome of Prochlorococcus and perhaps define this group from other closely related organisms, such as Synechococcus (Kettler et al, 2007). Thus, Prochlorococcus offers a useful model system in which to study how diversity among closely related organisms influences the collective iron metabolism of this abundant group.

\section{Iron and Prochlorococcus - what we know}

Before this study, most of our understanding of Prochlorococcus iron metabolism rests on the recognition of homologs to known iron-related genes in the Prochlorococcus genomes. Several iron-related genes are annotated in the twelve sequenced genomes (Figure 1) (Dufresne et al, 2003; Kettler et al, 2007; Rocap et al, 2003). Yet, the inferred functions of these genes do not present a cohesive picture of iron metabolism in Prochlorococcus. Much work on iron metabolism has been completed in other gram-negative bacteria, especially Escherichia coli. And, iron metabolism in some cyanobacteria has been investigated through culture-based and genomic approaches (Ferreira and Straus, 1994; Katoh et al, 2001; Kutzki, 1998; Michel, 1999; Singh et al, 2003; Singh and Sherman, 2000). These studies provide guidelines to the fundamental aspects of iron metabolism that we should expect to find in Prochlorococcus. In our assessment of the iron metabolism of Prochlorococcus, we expect to find proteins that require 
iron, facilitate iron transport, store iron, regulate iron homeostasis, and enable acclimation to low iron availability (Andrews et al, 2003).

In cyanobacteria, iron is best known for its role as a cofactor in photosynthetic electron transfer. In fact, 22 atoms of iron are considered necessary for the function of a complete photosynthetic apparatus including the photosystem II complex (PSII), photosystem I complex (PSI), the cytochrome $\mathrm{b}_{6} / \mathrm{f}$ complex, and soluble electron carriers like ferredoxins (Ferreira et al, 1994; Raven, 1988). Obviously, Prochlorococcus contains all necessary components for photosynthesis and for that reason its metabolism may depend greatly on iron availability. In addition, the Prochlorococcus core genome contains plastocyanin, which traditionally requires 1 additional atom of iron when the protein is in use during photosynthesis (Ferreira et al, 1994) but is predicted to contain copper in Prochlorococcus. Iron is also an important component of nitrogen metabolism. All cultured Prochlorococcus can assimilate ammonium and therefore require iron in the ferredoxin-dependent glutamate synthase (gltB). Iron is also necessary for nitrate assimilation but nitrate reductase has not been found in any sequenced Prochlorococcus isolate (Kettler et al, 2007; Rocap et al, 2003). Though, many of the low-light adapted ecotypes are capable of using nitrite as a sole $\mathrm{N}$ source and have nirA, a ferredoxin dependent-nitrite reductase. So, in Prochlorococcus, as in other cyanobacteria, iron is likely required for nitrogen assimilation. In addition, there are several predicted Fe-S proteins and ferredoxins present in the Prochlorococcus core genome which we expect contribute to its total iron requirement (Alm et al, 2005; Kettler et al, 2007).

A complete iron-uptake system has not yet been recognized in any marine cyanobacteria, which suggests that either the sequences of known iron-uptake systems have significantly diverged from their homologs in well-studied organisms, or that marine cyanobacteria utilize novel means of iron acquisition (Rocap et al, 2003; Webb et al, 2001). In some cyanobacteria, siderophores are used during conditions of low iron availability to enhance iron uptake, yet no recognizable siderophore synthesis genes are present in Prochlorococcus genomes (Goldman et al, 1983; Rocap et al, 2003). Many other gram negative bacteria, such as E. coli, are capable of assimilating iron from the siderophores of other organisms, but again, no siderophore acquisition systems are recognized in the Prochlorococcus genomes (Raymond et al, 2003; Rocap et al, 2003). Furthermore, there is no evidence that suggests a capability in Prochlorococcus of transporting ferrous iron (such as the $f e o A B$ system from $E$. coli) though this capability has been recognized in the single-celled nitrogen-fixing cyanobacterium Cyanothece sp. ATCC 51142 
(Welsh et al, 2008). Nor is there genomic evidence to suggest that Prochlorococcus is capable of reducing ferric iron to ferrous iron at the cell surface using a system similar to that employed in yeast or eukaryotic phytoplankton (Maldonado and Price, 2001) or extra-cellularly like E. coli (Cowart, 2002; Schröder et al, 2003; Vartivarian and Cowart, 1999).

Nonetheless, there are some homologs to known-iron transport systems in the Prochlorococcus core genome (Figure 1). The most obvious iron transport genes are the futABC genes encoding the substrate-binding, permease, ATP-binding component, and periplasmic substrate-binding protein of a putative $\mathrm{Fe}^{3+} \mathrm{ABC}$ transporter. futA is homologous to $a f u A$ in Synechocystis and to the iron-deficiency induced protein, idiA, which is present in other marine cyanobacteria and known to be expressed and translated during iron limitation (Webb et al, 2001). futC is homologous to $s f u C$ a known component of an iron ABC transporter in Serratia marcescens (Alm et al, 2005; Andrews et al, 2003). Several suf genes are present in the Prochlorococcus core genome and are also predicted to be involved in iron transport through their function in Fe-S assembly. In Synechocystis, several suf genes respond to iron-stress which suggests that they could also be involved in Prochlorococcus iron metabolism (Wang et al, 2004). Another interesting set of genes in the Prochlorococcus core genome encode a putative Mn transporter system and include PMM0601-PMM0603 (in MED4). Homologs in Yersinia pestis ( $y f e A B C$ ) are required for normal growth under low iron conditions but are repressed by both $\mathrm{Mn}$ and $\mathrm{Fe}$ (Bearden et al, 1998). Homologs in E. coli (sitABC) have greater affinity for Mn than Fe suggesting that they might be more relevant to Mn transport than Fe transport (Andrews et al, 2003). The affinity of these proteins for iron or manganese remains to be tested in Prochlorococcus. Transport across the outer-membrane is an energy-dependent process and strangely Prochlorococcus and Synechococcus genomes are missing the TonB-ExbB-ExbD system that enables transport of iron across the outer-membrane in other gram-negative bacteria (Andrews et al, 2003). Interestingly, gram-positive bacteria transport iron without use of the TonB-ExbB-ExbD system in favor of using ABC permeases and a lipoprotein attached to the exterior of the cell surface, and cyanobacteria cell walls appear to have both gram-positive and gram-negative properties (Andrews et al, 2003; Hoiczyk and Hansel, 2000).

The importance of storing, sequestering, or compartmentalizing iron within the cell is emphasized by the damage caused to proteins and nucleic acids by radical oxygen species produced via the Fenton reaction when free-iron exists in the cell (Touati, 2000). Ferritins, bacterioferritins, and Dps proteins are three types of iron storage proteins known among bacteria 
(Andrews et al, 2003). All three proteins assemble into multi-subunit spherical protein shells that contain ferric iron. Ferritins are present in both bacteria and eukaryotes. The Prochlorococcus core genome contains one ferritin (PMED4_08901) though MIT9313 and MIT9303 contain a second copy of ferritin (P9313_05881) (Figure 1). The haem-containing bacterioferritins are only observed in eubacteria and their role in iron storage is less well-defined than the ferritins. In fact, in E. coli, bacterioferritin mutants do not display aberrant phenotypes. Bacterioferritins are not present in the Prochlorococcus genomes (microbesonline.org). The third known iron-storing molecules are Dps-proteins, which are present only in prokaryotes. Though dps-proteins seem capable of iron storage in E. coli, it is not believed to be their principal function (Andrews et al, 2003). While the Prochlorococcus core genome does not contain any dps-like proteins, the genomes of two low-light adapted ecotypes, MIT9313 and MIT9303, do contain a dps-like protein (P9313_27811), which could play a role in iron storage.

The dependence of cellular metabolism on iron and the danger posed by free-iron within a cell argue for tight regulation of iron transport, storage, and use in the intracellular environment. The ferric uptake regulator, fur, was first identified in E. coli and is present and active in the iron regulation systems of many other organisms. Fur acts as a negative repressor of genes involved in iron transport and storage by repressing transcription when bound to iron. Fur appears to regulate at least 90 genes in E. coli (Hanke, 2001). Recently, the positive regulation of iron-containing proteins by fur, acting as an indirect activator when iron is abundant, was explained by its repression of a small RNA (RyhB) that negatively regulates genes encoding iron-containing proteins (Davis et al, 2005; Masse and Gottesman, 2002; Wilderman et al, 2004). Fur is present in two copies in the Prochlorococcus core genome (PMED4_11731 and PMED4_06871) and a third copy is present in MIT9313 and MIT9303 (P9313_06201) (Figure 1). Though several small RNAs have been identified in Prochlorococcus, an RhyB homolog has not yet been discovered (Steglich et al, 2008).

We also expect to find features in the Prochlorococcus genomes that are designed to help the cell cope with low iron availability. Aside from up-regulation of iron transporters during iron stress which we have discussed above, replacement of iron-containing proteins with non-iron containing substitutes is another mechanism cells use to lower their overall iron requirement. The iron-stress induced gene, isiB, encoding flavodoxin, is present in the Prochlorococcus core genome (Figure 1) and is known to replace the iron-requiring electron transfer protein ferredoxin during iron stress in diverse photosynthetic organisms (Rocap et al, 2003; Straus, 1994). The 
responsiveness of isiB to iron deprivation in Prochlorococcus has been tested in MED4, MIT9313, and SS120 and is clearly up-regulated during iron stress (Bibby et al, 2003). In the same study, components of the iron-rich PSI were down-regulated in response to iron deprivation in SS120 and MIT9313, but not MED4. While we do not know of an iron-free replacement for any components of the PSI complex, the study found that one copy of the light-harvesting complex protein gene ( $p c b)$ in both SS120 (pcbC) and MIT9313 (pcbB) responded strongly to iron-deprivation and appears to form a ring around PSI during low iron availability, in effect compensating for the down-regulation of the components of the PSI complex (Bibby et al, 2003). Interestingly, the high-light adapted Prochlorococcus ecotypes contain only one copy of $p c b$ that in MED4, at least, did not respond to iron deprivation (Bibby et al, 2003).

Another well-characterized consequence of iron stress in many organisms is oxidative stress (Touati, 2000). Prochlorococcus genomes contain many copies of high-light inducible proteins (hlips) that are thought to be involved in responding to oxidative stress (Kettler et al, 2007). So far, expression of hlips has been observed during light stress, nitrogen stress, and phage infection so a role for them in responding to oxidative stress induced by iron starvation is likely.

Bacterioferritin co-migratory proteins are another class of proteins that are thought to be involved in protecting cells from oxidative stress (Jeong et al, 2000). A putative bacterioferritin comigratory protein is present in the Prochlorococcus core genome.

\section{Prochlorococcus and iron at the cellular level}

As a single-celled free-living organism with a minimal genome Prochlorococcus embodies a relatively simple link between iron biogeochemical cycles in the open ocean and global patterns and dynamics of primary productivity. We know that Prochlorococcus requires iron to survive. And, we know that Prochlorococcus experiences iron-limitation of growth rate in the Equatorial Pacific (Cavender-Bares et al, 1999; Mann et al, 2000). These observations suggest that iron availability plays an important role in determining the distribution, abundance, and diversity of Prochlorococcus. The major goal of this thesis is to describe the relationship between Prochlorococcus and iron at the cellular level so that we may improve our understanding of the role iron plays in governing Prochlorococcus ecology.

Prochlorococcus is an ideal system in which to study the link between iron and primary productivity for more reasons than its abundance and ubiquity in the open ocean. Though closely related by phylogeny, Prochlorococcus ecotypes are diverse in many aspects of their metabolism 
and offer a platform from which to study how diversity in iron metabolism arises. As Prochlorococcus ecotypes are optimally adapted to different nitrogen, phosphorus, and light regimes, there is an opportunity to study how iron metabolism intersects with and overlaps with other aspects of cellular metabolism. Furthermore, the availability of twelve Prochlorococcus genomes provides an opportunity to understand how iron metabolism is encoded in the core genome or in more variable regions of the genome. Finally, the abundance of Prochlorococcus sequences in environmental metagenomic databases, and our ability to measure the physiological parameters of individual Prochlorococcus cells via flow cytometry, allows us to apply what we learn in the laboratory concerning Prochlorococcus to natural populations in the open ocean.

First, we examine the iron physiology of the high-light adapted ecotype MED4 and lowlight adapted ecotype MIT9313 (Chapter 2). By acclimating axenic cultures to a range of iron concentrations we ask: What is the minimum concentration of iron required for growth? Over the range of iron concentrations, cultures will also be acclimated to a high- and low- light intensity. Thus, we address the complex interaction between light and iron in photosynthesis by posing the question: How does light availability influence the iron physiology of

Prochlorococcus? Finally, we will discuss how intracellular iron concentrations vary between the two Prochlorococcus ecotypes and change with light availability.

Next, we examine the molecular mechanisms that support the difference we see in the iron physiologies of MED4 and MIT9313 by asking: What gene transcripts in MED4 and MIT9313 are responsive to changes in iron availability? (Chapter 3). To answer this question, we examine gene expression over a time-course of iron starvation and rescue in both strains. Because similar experiments have been conducted that assess the MED4 and MIT9313 wholegenome response to other stress conditions, such as nitrogen starvation, phosphorous starvation, light shifts, and phage infection, we are capable of addressing the question: How does the wholegenome response to iron starvation compare to other stress responses? Finally, having identified isiB (flavodoxin) as a sound marker of iron stress in Prochlorococcus, we describe plans to examine the extent to which distinct Prochlorococcus ecotypes, and Synechococcus, are afflicted by iron stress over natural gradients of iron concentrations and speciation in the open ocean (Chapter 4).

This thesis represents the first detailed and multi-scale study on the role of iron in Prochlorococcus ecology and diversity. Our approach entailed physiological studies in the laboratory and field, chemical analysis, whole-genome expression studies, and comparative 
genomics. This work allows us to begin to understand how iron metabolism has shaped the Prochlorococcus core genome during evolution and how part of the diversity and success of Prochlorococcus in today's ocean is due to diversity in its iron metabolism. Thus, the thesis provides a new set of insights towards understanding the environmental factors that control the abundance and determine the success of Prochlorococcus in the open ocean.

\section{References}

Alm EJ, Huang KH, Price MN, Koche RP, Keller K, Dubchak IL, Arkin AP (2005) The MicrobesOnline Web set for comparative genomics. Genome Research 15: 1015-1022.

Andrews SC, Robinson AK, Rodriguez-Quinones F (2003) Bacterial iron homeostasis. FEMS Microbiology Reviews 27: 215-237.

Barbeau K (2006) Photochemistry of Organic Iron(III) Complexing Ligands in Oceanic Systems. Photochemistry and Photobiology 82: 1505-1516.

Bearden SW, Staggs TM, Perry RD (1998) An ABC Transporter System of Yersinia pestis Allows Utilization of Chelated Iron by Escherichia coli SAB11. J Bacteriol 180: 1135-1147.

Bibby TS, Mary I, Nield J, Partensky F, Barber J (2003) Low-light-adapted Prochlorococcus species possess specific antennae for each photosystem. Nature 424: 1051-1054.

Boyd PW, Jickells T, Law CS, Blain S, Boyle EA, Buesseler KO, Coale KH, Cullen JJ, de Baar HJW, Follows M, Harvey M, Lancelot C, Levasseur M, Owens NPJ, Pollard R, Rivkin RB, Sarmiento J, Schoemann V, Smetacek V, Takeda S, Tsuda A, Turner S, Watson AJ (2007) Mesoscale Iron Enrichment Experiments 1993-2005: Synthesis and Future Directions. Science 315: 612-617.

Boye M, Aldrich A, van den Berg CMG, de Jong JTM, Nirmaier H, Veldhuis M, Timmermans KR, de Baar HJW (2006) The chemical speciation of iron in the north-east Atlantic Ocean. Deep Sea Research Part I: Oceanographic Research Papers 53: 667-683.

Bruland KW, Lohan MC, Heinrich DH, Karl KT (2003) Controls of Trace Metals in Seawater. In Treatise on Geochemistry, pp 23-47. Oxford: Pergamon.

Cavender-Bares KK, Mann EL, Chisholm SW, Ondrusek ME, Bidigare RR (1999) Differential response of equatorial Pacific phytoplankton to iron fertilization. Limnology and Oceanography 44: 237-246.

Coleman ML, Chisholm SW (2007) Code and context: Prochlorococcus as a model for cross-scale biology. Trends in Microbiology 15: 398-407.

Coleman ML, Sullivan MB, Martiny AC, Steglich C, Barry K, DeLong EF, Chisholm SW (2006) Genomic Islands and the Ecology and Evolution of Prochlorococcus. Science 311: 1768-1770.

Cowart RE (2002) Reduction of iron by extracellular iron reductases: implications for microbial iron acquisition. Archives of Biochemistry and Biophysics 400: 273-281.

Dauphas N, van Zuilen M, Wadhwa M, Davis AM, Marty B, Janney PE (2004) Clues from Fe Isotope Variations on the Origin of Early Archean BIFs from Greenland. Science 306: 2077-2080.

Davis BM, Quinones M, Pratt J, Ding Y, Waldor MK (2005) Characterization of the Small Untranslated RNA RyhB and Its Regulon in Vibrio cholerae. J Bacteriol 187: 4005-4014.

Duce RA, Tindale NW (1991) Atmospheric Transport of Iron and its Deposition in the Ocean. Limnology and Oceanography 36: 1715-1726.

Dufresne A, Salanoubat M, Partensky F, Artiguenave F, Axmann I, Barbe V, Duprat S, Galperin M, Koonin E, Le Gall F, Makarova K, Ostrowski M, Oztas S, Robert C, Rogozin I, Scanlan D, Tandeau de Marsac N, Weissenbach J, Wincker P, Wolf Y, Hess W (2003) Genome sequence of the cyanobacterium Prochlorococcus marinus SS120, a nearly minimal oxyphototrophic genome. Proceedings of the National Academy of Sciences 100: 10020 - 10025.

Ferreira F, Straus NA (1994) Iron deprivation in cyanobacteria. Journal of Applied Phycology 6: 199-210.

Goldman SJ, Lammers PJ, Berman MS, Sanders-Loehr J (1983) Siderophore-Mediated Iron Uptake in Different Strains of Anabaena sp. Journal of Bacteriology 156: 1144-1150.

Hanke K (2001) Iron and metal regulation in bacteria. Current Opinion in Microbiology 4: 172-177. 
Hoiczyk E, Hansel A (2000) Cyanobacterial Cell Walls: News from an Unusual Prokaryotic Envelope. J Bacteriol 182: 1191-1199.

Hunter KA, Boyd PW (2007) Iron-binding ligands and their role in the ocean biogeochemistry of iron. Environmental Chemistry 4: 221-232.

Hurst MP, Bruland KW (2007) An investigation into the exchange of iron and zinc between soluble, colloidal, and particulate size-fractions in shelf waters using low-abundance isotopes as tracers in shipboard incubation experiments. Marine Chemistry 103: 211-226.

Jeong W, Cha M-K, Kim I-H (2000) Thioredoxin-dependent Hydroperoxide Peroxidase Activity of Bacterioferritin Comigratory Protein (BCP) as a New Member of the Thiol-specific Antioxidant Protein (TSA)/Alkyl Hydroperoxide Peroxidase C (AhpC) Family. J Biol Chem 275: 2924-2930.

Jickells TD, An ZS, Andersen KK, Baker AR, Bergametti G, Brooks N, Cao JJ, Boyd PW, Duce RA, Hunter KA, Kawahata H, Kubilay N, laRoche J, Liss PS, Mahowald N, Prospero JM, Ridgwell AJ, Tegen I, Torres R (2005) Global Iron Connections Between Desert Dust, Ocean Biogeochemistry, and Climate. Science 308: 67-71.

Johnson KS, Gordon RM, Coale KH (1997) What controls dissolved iron concentrations in the world ocean? Marine Chemistry 57: 137-161.

Johnson ZI, Zinser ER, Coe A, McNulty NP, Woodward EMS, Chisholm SW (2006) Niche Partitioning Among Prochlorococcus Ecotypes Along Ocean-Scale Environmental Gradients. Science 311: 1737-1740.

Jordan P, Fromme P, Witt HT, Klukas O, Saenger W, Krausz N (2001) Three-dimensional structure of

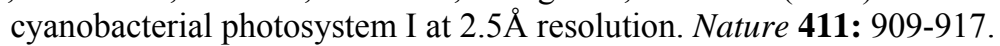

Katoh H, Hagino N, Grossman AR, Ogawa T (2001) Genes Essential to Iron Transport in the Cyanobacterium Synechocystis sp. Strain PCC 6803. Journal of Bacteriology 183: 2779-2784.

Kettler GC, Martiny AC, Huang K, Zucker J, Coleman ML, Rodrigue S, Chen F, Lapidus A, Ferriera S, Johnson J, Steglich C, Church GM, Richardson P, Chisholm SW (2007) Patterns and Implications of Gene Gain and Loss in the Evolution of Prochlorococcus. PLoS Genetics 3: e231.

Kump L (2005) OCEAN SCIENCE: Ironing Out Biosphere Oxidation. Science 307: 1058-1059.

Kutzki C, Masepohl, B., Böhme, H. (1998) The isiB gene encoding flavodoxin is not essential for photoautotrophic iron limited growth of the cyanobacterium Synechocystis sp. strain PCC 6803. FEMS Microbiology Letters 160: 231-235.

Mahowald N, Kohfeld K, Hansson M, Balkanski Y, Harrison SP, Prentice IC, Schulz M, Rodhe H (1999) Dust sources and deposition during the last glacial maximum and current climate: A comparison of model results with paleodata from ice cores and marine sediments. J Geophys Res 104.

Maldonado MT, Price NM (2001) Reduction and transport of organically bound iron by Thalassiosira Oceanica (Bacillariophyceae) Journal of Phycology 37: 298-310.

Mann EL, Chisholm SW (2000) Iron limits the cell division rate of Prochlorococcus in the eastern equatorial Pacific. Limnology and Oceanography 45: 1067-1076.

Martin JH, Fitzwater SE, Gordon RM (1990a) Iron deficiency limits phytoplankton growth in Antarctic waters. Global Biogeochemical Cycles 4: 5-12.

Martin JH, Gordon RM (1988) Northeast Pacific iron distributions in relation to phytoplankton productivity. Deep-Sea Research 35: 177-196.

Martin JH, Gordon RM, Fitzwater SE (1990b) Iron in Antarctic waters. Nature 345: 156-158.

Masse E, Gottesman S (2002) A small RNA regulates the expression of genes involved in iron metabolism in Escherichiacoli. Proceedings of the National Academy of Sciences 99: 4620-4625.

Michel K, Krüger, F., Pühler, A., and Pistorius, E.K. (1999) Molecular characterization of idiA and adjacent genes in the cyanobacteria Synechcoccus sp. strains PCC 6301 and PCC 7942. Microbiology 145: 1473-1484.

Moore JK, Doney SC, Lindsay K (2004) Upper ocean ecosystem dynamics and iron cycling in a global three-dimensional model. Global Biogeochemical Cycles 18: GB4028.

Moore LR, Chisholm SW (1999) Photophysiology of the Marine Cyanobacterium Prochlorococcus: Ecotypic Differences among Cultured Isolates. Limnology and Oceanography 44: 628-638. 
Moore LR, Post AF, Rocap G, Chisholm SW (2002) Utilization of Different Nitrogen Sources by the Marine Cyanobacteria Prochlorococcus and Synechococcus. Limnology and Oceanography 47: 989-996.

Nishioka J, Takeda S, de Baar HJW, Croot PL, Boye M, Laan P, Timmermans KR (2005) Changes in the concentration of iron in different size fractions during an iron enrichment experiment in the open Southern Ocean. Marine Chemistry 95: 51-63.

Nishioka J, Takeda S, Wong CS, Johnson WK (2001) Size-fractionated iron concentrations in the northeast Pacific Ocean: distribution of soluble and small colloidal iron. Marine Chemistry 74: 157-179.

Partensky F, Hess WR, Vaulot D (1999) Prochlorococcus, a Marine Photosynthetic Prokaryote of Global Significance. Microbiol Mol Biol Rev 63: 106-127.

Raven JA (1988) The Iron and Molybdenum use Efficiencies of Plant Growth with Different Energy, Carbon and Nitrogen Sources. New Phytologist 109: 279-287.

Raymond KN, Dertz EA, Kim SS (2003) Enterobactin: An archetype for microbial iron transport. Proceedings of the National Academy of Sciences of the United States of America 100: 3584-3588.

Rocap G, Larimer FW, Lamerdin J, Malfatti S, Chain P, Ahlgren NA, Arellano A, Coleman M, Hauser L, Hess WR, Johnson ZI, Land M, Lindell D, Post AF, Regala W, Shah M, Shaw SL, Steglich C, Sullivan MB, Ting CS, Tolonen A, Webb EA, Zinser ER, Chisholm SW (2003) Genome divergence in two Prochlorococcus ecotypes reflects oceanic niche differentiation. Nature 424: 1042-1047.

Rue EL, Bruland KW (1995) Complexation of iron(III) by natural organic ligands in the Central North Pacific as determined by a new competitive ligand equilibration/adsorptive cathodic stripping voltammetric method. Marine Chemistry 50: 117-138.

Schröder I, Johnson E, Vries S (2003) Microbial ferric iron reductases. FEMS Microbiology Reviews 27: 427-447.

Singh AK, McIntyre LM, Sherman LA (2003) Microarray Analysis of the Genome-Wide Response to Iron Deficiency and Iron Reconstitution in the Cyanobacterium Synechocystis sp. PCC 6803. Plant Physiology 132: 1825-1839.

Singh AK, Sherman LA (2000) Identification of Iron-Responsive, Differential Gene Expression in the Cyanobacterium Synechocystis sp. Strain PCC 6803 with a Customized Amplification Library. Journal of Bacteriology 182: 3536-3543.

Steglich C, Futschik ME, Lindell D, Voss B, Chisholm SW, Hess WR (2008) The Challenge of Regulation in a Minimal Photoautotroph: Non-Coding RNAs in Prochlorocococcus. PLoS Genetics 4: 1-14.

Straus NA (1994) Iron deprivation: physiology and gene regulation. In The Molecular Biology of Cyanobacteria, Bryant DA (ed), pp 731-750. Dordrecht, The Netherlands: Kluwer Academic Publishers.

Sullivan MB, Waterbury JB, Chisholm SW (2003) Cyanophages infecting the oceanic cyanobacterium Prochlorococcus. Nature 424: 1047-1051.

Tegen I, Fung I (1995) Contribution to the atmospheric mineral aerosol load from land surface modification. J Geophys Res 100.

Touati D (2000) Iron and Oxidative Stress in Bacteria. Archives of Biochemistry and Biophysics 373: 1-6.

van den Berg CMG (1995) Evidence for organic complexation of iron in seawater. Marine Chemistry 50: 139-157.

Vartivarian SE, Cowart RE (1999) Extracellular Iron Reductases: Identification of a New Class of Enzymes by Siderophore-Producing Microorganisms. Archives of Biochemistry and Biophysics 364: 75-82.

Wang T, Shen G, Balasubramanian R, McIntosh L, Bryant DA, Golbeck JH (2004) The sufR Gene (sll0088 in Synechocystis sp. Strain PCC 6803) Functions as a Repressor of the sufBCDS Operon in IronSulfur Cluster Biogenesis in Cyanobacteria. J Bacteriol 186: 956-967.

Webb EA, Moffett JW, Waterbury JB (2001) Iron Stress in Open-Ocean Cyanobacteria (Synechococcus, Trichodesmium, and Crocosphaera spp.): Identification of the IdiA Protein. Appl Environ Microbiol 67: 5444-5452.

Weber L, Volker C, Oschlies A, Burchard H (2007) Iron profiles and speciation of the upper water column at the Bermuda Atlantic Time-series Study site: a model based sensitivity study. Biogeosciences 4: 689-706. 
Welsh EA, Liberton M, Stöckel J, Loh T, Elvitigala T, Wang C, Wollam A, Fulton RS, Clifton SW, Jacobs JM, Aurora R, Ghosh BK, Sherman LA, Smith RD, Wilson RK, Pakrasi HB (2008) The genome of Cyanothece 51142, a unicellular diazotrophic cyanobacterium important in the marine nitrogen cycle. Proceedings of the National Academy of Sciences 105: 15094-15099.

Wilderman PJ, Sowa NA, FitzGerald DJ, FitzGerald PC, Gottesman S, Ochsner UA, Vasil ML (2004) Identification of tandem duplicate regulatory small RNAs in Pseudomonas aeruginosa involved in iron homeostasis. Proceedings of the National Academy of Sciences of the United States of America 101: 9792-9797.

Wu J, Boyle E, Sunda W, Wen L-S (2001) Soluble and Colloidal Iron in the Oligotrophic North Atlantic and North Pacific. Science 293: 847-849.

Zinser ER, Coe A, Johnson ZI, Martiny AC, Fuller NJ, Scanlan DJ, Chisholm SW (2006) Prochlorococcus Ecotype Abundances in the North Atlantic Ocean As Revealed by an Improved Quantitative PCR Method. Appl Environ Microbiol 72: 723-732. 



\title{
CHAPTER TWO
}

\section{Prochlorococcus iron physiology and ratios of intracellular trace metals}

\author{
Anne W. Thompson ${ }^{1}$
}

Sallie W. Chisholm ${ }^{2}$

Mak Saito ${ }^{3}$

\footnotetext{
${ }^{1}$ MIT-WHOI Joint Program in Biological Oceanography

${ }^{2}$ MIT Departments of Civil and Environmental Engineering and Biology

${ }^{3}$ Woods Hole Oceanographic Institution - Department of Marine Chemistry and Geochemistry
} 


\begin{abstract}
The abundance of Prochlorococcus in the global ocean is due its diversity, which collectively allows it to thrive over a large range of environmental conditions. We know that Prochlorococcus requires iron and can be limited by iron availability in some regimes of the open ocean. Yet, we do not understand how gradients in iron availability in the open ocean have shaped the ecology or controlled the distribution of this abundant primary producer. Ecosystem models strive to numerically represent the complexities of both primary productivity and iron biogeochemical cycling. Thus, understanding these connections in the context of

Prochlorococcus would be very valuable. Here, we grow a high-light adapted and a low-light adapted Prochlorococcus ecotype over a range of iron concentrations and light intensities to understand its minimum iron requirement. We find that the low-light adapted ecotype, MIT9313ax, is capable of growth at lower iron concentrations than high-light adapted ecotype, MED4ax. Furthermore, we observe that Fe' concentrations in the field are capable of sustaining Prochlorococcus growth at concentrations far lower than can support growth in culture.
\end{abstract}

\title{
Introduction
}

Ecosystem modeling is a powerful method of probing our understanding of the link between the specific metabolic requirements of phytoplankton and global patterns of primary productivity. Yet, so far, these models have been forced to ignore the effect of iron availability on primary productivity or, parameterize phytoplankton in terms of their ability to assimilate different types of iron due to lack of data and the difficulty of representing diverse nutrient physiologies in a numeric model (Aumont et al, 2003; Moore et al, 2004; Oschlies and Garcon, 1998). Follows et al. 2007 take a different approach to ecosystem modeling where a model ocean is seeded with phytoplankton of diverse nitrogen physiologies (Follows et al, 2007). We expect that this type of model may be appropriate for representing the diversity of phytoplankton iron physiologies in the ocean. Yet, data that could inform such a model for iron are still scarce. These ecosystem models can be strengthened and their predictive capabilities improved with data on the iron requirements of abundant phytoplankton types, such as Prochlorococcus, the speciation of iron in the open ocean, and the forms of iron that are available for uptake by phytoplankton.

\section{Bioavailability of iron in the open ocean}

One predominant question in the field of trace metal biogeochemistry is: What form of iron is taken up by phytoplankton? Researchers are beginning to understand that the answer to this question is very complex, even in systems of defined media and clonal cultures in the laboratory. Traditionally, iron uptake rates by phytoplankton in culture were thought to depend on the concentration of the sum of inorganic hydrolysis species of iron (Fe'), as described in the Fe' 
model (Hudson and Morel, 1990). In an oxic seawater medium buffered with an organic ligand, for example EDTA, iron would exist in a complexed form with EDTA, bound to oxygen as iron hydroxides $\left(\mathrm{Fe}(\mathrm{OH})_{\mathrm{x}}\right)$, and in the free ferric form $\left(\mathrm{Fe}^{3+}\right)$. Only the combined pools of $\mathrm{Fe}(\mathrm{OH})_{\mathrm{x}}$ and $\mathrm{Fe}^{3+}$ make up $\mathrm{Fe}^{\prime}$ and would be considered as biologically available under the Fe' model (Hudson et al, 1990). The buffering properties of EDTA (or another similar organic chelator) would keep Fe' concentrations constant during phytoplankton growth and serves as a tool to control the concentration of bioavailable iron. Recently, researchers discovered that at very low Fe' concentrations, Thalassiosira oceanica could reduce iron bound to the strong organic ligand DFB at the cell surface for uptake. Thus, this diatom relied on organically-bound iron rather than Fe' to satisfy its iron requirement (Maldonado and Price, 2001). Now, a new model reconciles the existing Fe' model with the evidence of cell surface iron reduction in eukaryotic phytoplankton (Shaked et al, 2005). The new model requires iron reduction for uptake of any iron species and emphasizes the importance of ligand concentration and conditional stability constants in determining which iron species are available for uptake by a given type of eukaryotic phytoplankton (Shaked et al, 2005). It is unclear if prokaryotic phytoplankton are also capable of reduction of iron from organic ligands at the cell surface.

We expect the rules that describe the bioavailability of iron and its speciation in the natural environment of the open ocean to be significantly more complex than in defined media. Since the realization that iron availability could drastically influence primary productivity we have learned a great deal about iron in the ocean. Importantly, it is now clear that two classes of organic ligands $\left(\mathrm{L}_{1}\right.$ and $\left.\mathrm{L}_{2}\right)$ bind most of the iron in seawater and make a case for the importance of ligand-bound iron species to the microbial community (Rue and Bruland, 1995; van den Berg, 1995; Wu and Luther, 1995). Through the work of several additional studies, it appears that the strong binding ligands, $\mathrm{L}_{1}$, may be responsible for Fe(III) speciation in the mixed layer while the weaker ligands, $\mathrm{L}_{2}$, may influence iron speciation in the deep ocean (Hunter and Boyd, 2007). Also, the organic ligand categories $\mathrm{L}_{1}$ and $\mathrm{L}_{2}$ appear to be present in both the colloidal (molecular diameter of $0.02 \mu \mathrm{m}$ to $0.4 \mu \mathrm{m})$ and soluble $(<0.02 \mu \mathrm{m})$ size classes of dissolved iron (less than $0.4 \mu \mathrm{m}$ in molecular diameter) (Wu et al, 2001). Interestingly, the vertical profile of soluble iron resembles a nutrient-type profile similar to that of major nutrients phosphate and nitrogen and the dissolved pool of iron. Colloidal iron, displays a deviant trend, showing a surface maximum and a minimum in the upper nutricline (Wu et al, 2001). Iron enrichment experiments in the Southern Ocean and in coastal waters of Canada demonstrate that colloidal iron is removed more easily 
from the surface waters during phytoplankton blooms than particulate and soluble fractions. In addition, during the phytoplankton bloom the concentration of colloidal iron appears to be the most dynamic of the group, suggesting the use of colloidal iron by phytoplankton (Nishioka et al, 2005; Nishioka et al, 2001).

Light also has the potential to influence iron speciation in the euphotic zone where phytoplankton live and adds another layer of complexity to the picture of iron availability and iron acquisition strategies in the open ocean. A chemical model developed by Weber et al. 2007 demonstrates fluctuations in the concentrations of Fe(III)', Fe(II)', iron bound to organic ligands, and colloidal iron over the diel cycle. These diel dynamics in iron speciation are due to the photochemical reduction of Fe(III) bound to organic ligands (Weber et al, 2007). It will be very interesting to validate this model in the open ocean and characterize the response, if any, of phytoplankton to changing iron availability over the diel cycle.

\section{Iron acquisition and uptake rates}

A second pressing question in the field of trace metal biogeochemistry is: What strategies do phytoplankton use to acquire enough iron to meet their metabolic requirements? Answering this question entails becoming familiar with the physical, chemical, and physiological traits that control the uptake of iron and other trace metals by phytoplankton. At the most simple level, cell size has been shown to be important in dictating iron uptake rates in phytoplankton. At high concentrations of iron, iron uptake is limited by ligand-exchange kinetics at the cell surface, i.e. the number of iron transporters on the cell surface and the rate at which iron can react with a single transporter (Morel et al, 1991; Sunda and Huntsman, 1995). Conversely, at low concentrations of iron, diffusion limits uptake-rate (Hudson, 1998; Morel et al, 1991). The importance of cell size in determining iron uptake rates is reflected in a study on several species of coastal and oceanic diatoms, dinoflagellates, and coccolithophores. These diverse species share iron uptake rates when normalized to cell surface area, despite the dramatic differences in iron concentrations between the coastal and oceanic waters to which they are adapted (Sunda and Huntsman, 1995; Sunda and Huntsman 1997. For smaller cells, ligand-exchange kinetics are more likely to limit iron uptake rates because the number of ligands on the cell surface is limited by surface area and competition for space with other membrane proteins. Though ligandexchange kinetics limit iron uptake in larger cells as well, diffusion limitation is more likely to 
inhibit iron uptake in large cells than in small cells due to the increased surface area to volume ratio of the former (Morel et al, 1991; Sunda and Huntsman, 1995).

In addition to examining the relationship between iron uptake rate, iron concentration, and cell size, Sunda and Huntsman (1995 and 1997) addressed the relationship between growth rate, iron quota (Fe: C), and light availability in species of diatoms and dinoflagellates. Because of the heavy involvement of iron in photosynthetic electron transport, cells growing at low irradiances were expected to become iron limited at higher iron concentrations than cells growing at high irradiances. Contrary to this expectation, Sunda and Huntsman (1997) found that the concentration of iron that induced iron limitation in a given species of phytoplankton is independent of light intensity. This unexpected result was explained by the idea that cells are taking up iron at the maximum rates permitted by the physics and chemistry of iron transporters and the media. So, cells growing at high light or at low light would transport iron at the same rates. Given the slower growth rate of low-light acclimated cells, cells growing at low light would accumulate higher cellular Fe: $\mathrm{C}$ ratios, which would allow the production of additional photosystem complexes for adaptation to low light intensities (Sunda and Huntsman, 1997). Investigating the links between light, iron concentration, growth rate, Fe: $\mathrm{C}$ and iron uptake in phytoplankton suggests mechanisms by which marine phytoplankton adapt to different iron regimes. Phytoplankton could use several different strategies to adapt to low iron availability. They could increase their surface area to biomass ratio by becoming smaller, decrease their growth rate allowing accumulation of iron (higher Fe: C), and/or reduce the amount of iron required (iron quota) to maintain maximal growth rate (Sunda and Huntsman, 1995).

Elucidating the molecular mechanisms of iron uptake is also critical towards understanding how phytoplankton satisfy their cellular iron requirements. Results of recent sequencing projects reveal genomic differences among phytoplankton that probably contribute to their iron physiologies. We currently know of several iron acquisition systems in marine phytoplankton that are tailored to take advantage of different forms of iron. For example, Prochlorococcus and Synechococcus contain homologs of an iron ABC transporter system (futABC) characterized in Synechocystis and Synechococcus that functions to bind iron (III) in the periplasm, yet are missing genes that encode proteins that provide energy to outer-membrane transporters (see Chapter 1) (Katoh et al, 2001; Kettler et al, 2007; Rocap et al, 2003; Singh et al, 2000; Sunda and Huntsman 1995; Webb et al, 2001). The recently sequenced coastal Synechococcus CC9311 and Cyanothece 51142 contain the feoAB genes used to transport iron(II) (Palenik et al. 2006; 
Welsh et al, 2008). And, recently diatoms have been shown to reduce iron(III) at the cell surface for transport (Maldonado et al, 2001).

\section{Prochlorococcus iron physiology}

In this study, Prochlorococcus iron physiology was assessed by measuring the minimum iron concentration required for growth of two strains of Prochlorococcus and their cellular metal quotas over a range of iron concentrations and light intensities. We chose axenic Prochlorococcus strains MED4ax and MIT9313ax because they are physiologically and phylogenetically distinct, represent the high-light adapted and low-light adapted ecotypes, and may demonstrate the range of iron physiologies possible in Prochlorococcus. Specifically, we asked: (1) What is the minimum iron concentration required for growth? (2) How does the minimum iron concentration required for growth vary between Prochlorococcus ecotypes and conditions of light availability? (3) How does iron concentration for growth in the laboratory compare to concentrations of iron that support Prochlorococcus growth in the field? (4) Finally, how does ecotype, light availability, and iron availability influence intracellular ratios of trace metals in Prochlorococcus?

\section{Materials and Methods}

Cultures and growth conditions - Axenic Prochlorococcus strains MED4ax and MIT9313ax were obtained from the Chisholm Laboratory culture collection. These strains were chosen in order to study how iron physiology varies between the closely related but physiologically distinct ecotypes of the Prochlorococcus group. Only axenic strains were studied to eliminate the influence of heterotrophic bacteria on growth rates or metal quota measurements.

Natural-seawater based PRO99 medium was modified for trace metal clean work (PRO99-TM) (Saito et al, 2002). Seawater for the media was obtained from the oligotrophic Atlanic Ocean onboard the R/V Seward Johnson in 2006. Modifications of PRO99 include using seawater collected under trace-metal clean conditions, microwave sterilization instead of autoclaving, a 10-fold increase in the EDTA concentration to $11.7 \mu \mathrm{M}$, and chelex treatment of major nutrients (Price et al, 1988; Saito et al, 2002). Table 1 presents the concentrations and source of all nutrients in PRO99-TM. The effectiveness of microwave sterilization was shown through purity tests using the Marine Purity Test Broth (MPTB) and PROMM purity test broths (data not shown) and is supported in previously published work (Keller et al, 1988; Moore et al, 
2007; Saito et al, 2002). All media preparations, transfers, and sampling was carried out under level-100 trace metal clean conditions in the Saito Laboratory at the Woods Hole Oceanographic Institution. All vessels were polycarbonate tubes or bottles that were soaked 12 hours in each $0.1 \%$ citranox (Alconox), $10 \% \mathrm{HCl}$ (Baker Instra-analyzed), and $\mathrm{pH} 2 \mathrm{Milli}-\mathrm{Q}$ water before use.

All experiments were conducted under continuous light and at $25^{\circ} \mathrm{C}$ in a walk-in incubator at the Woods Hole Oceanographic Institution. Light intensities were chosen to explore the effect of light availability on iron physiology. A common light intensity $\left(20 \mu \mathrm{Em}^{-2} \mathrm{~s}^{-1}\right)$ for MED4ax and MIT9313ax was chosen to attempt to achieve the same growth rate in both strains. A second light intensity was chosen for each strain so that each strain would be growing at a nearoptimal and sub-optimal light intensity.

\begin{tabular}{|l|l|l|}
\hline \multicolumn{2}{|c|}{ Table 1: Iron-replete PRO99-TM media composition (Saito et al, 2002) } \\
\hline \hline \multicolumn{1}{|c|}{ Nutrient } & \multicolumn{1}{|c|}{ Manufacturer and grade } & \multicolumn{1}{c|}{ Final concentration $(\mu \mathrm{M})$} \\
\hline \hline $\mathrm{NaH}_{2} \mathrm{PO}_{4}$ & Mallinkrodt ACS grade & 50 \\
\hline $\mathrm{NH}_{4} \mathrm{Cl}$ & Mallinkrodt ACS grade & 800 \\
\hline $\mathrm{EDTA}$ & Sigma Ultra $\sim 99 \%$ & 11.7 \\
\hline $\mathrm{FeCl}$ & Mallinkrodt Analytic & 1.17 \\
\hline $\mathrm{MnCl}_{2}$ & Sigma BioUltra $>99.0 \%$ & 0.090 \\
\hline $\mathrm{Na}_{2} \mathrm{MoO}_{4}$ & Sigma ACS $>99 \%$ & 0.003 \\
\hline $\mathrm{Na}_{2} \mathrm{SeO}_{3}$ & Sigma $99 \%$ & 0.010 \\
\hline $\mathrm{ZnCl}_{2}$ & Fluka $>99.5 \%$ & 0.010 \\
\hline $\mathrm{CoCl}_{2}$ & Mallinkrodt Analytic & 0.010 \\
\hline $\mathrm{NiCl}_{2}$ & Mallinkrodt Analytic & 0.010 \\
\hline
\end{tabular}

Iron-starvation and steady-state growth - MED4ax and MIT9313ax were transferred seven times to achieve steady-state growth rates at each iron concentration and light intensity. The media used for each iron concentration was made in one batch at the beginning of the experiment and used to transfer both MED4ax and MIT9313ax at each of the different light levels, in 28ml tubes, in order to better control our comparison between MED4ax and MIT9313ax. The inoculum for each strain and light intensity was obtained by centrifuging a light-acclimated mid-log phage culture at 8500 rpm for 10 minutes and rinsing two-times with un-amended sterile seawater. During the final rinse, cultures were re-suspended in a small volume of un-amended seawater and inoculated into duplicate tubes of media over the range of iron concentrations. In both experiments, growth was monitored using changes in bulk fluorescence measured on a TD700 fluorometer (Turner Designs). Cultures were transferred to fresh media when they reached mid-log phase measured by changes in bulk fluorescence. All cultures were transferred finally into $400 \mathrm{ml}$ of media in 
duplicate. Growth was again monitored using changes in bulk fluorescence and flow cytometry samples were collected to measure cellular growth-rates. During mid-log phase, cultures were sampled for cell number, intracellular trace metal concentrations, carbon, dissolved organic carbon, and chlorophyll under trace-metal clean conditions.

Trace metal concentrations - Iron concentrations ranged between no added iron and $1 \mu \mathrm{M}$ total added iron. Concentrations of dissolved iron (Fe') were calculated based on solubility constants from Morel and Herring 2003. Background levels of iron in the media were measured using an $\mathrm{Mg}$ co-precipitation method and inductively-coupled plasma mass spectrometry (ICP-MS) (Saito and Schneider, 2006).

Measuring cell number - Flow cytometry samples were collected from cultures to measure growth rate by changes in cell number and to measure cell size (relative forward angle light scatter) and relative red fluorescence per cell. Samples were collected in $1 \mathrm{ml}$ volumes and fixed for 10 minutes in the dark in $0.12 \%$ glutaraldehyde (Tousmis) and flash frozen in liquid nitrogen. Samples were run on a flow sorter (Cytopeia Influx) along with $2 \mu \mathrm{m}$ FluorescBrite standard beads (Polysciences, Inc) as an internal standard.

Carbon per cell - Carbon samples were collected for MED4ax during mid-log phase growth in the seventh transfer in each iron concentration and light intensity. $100 \mathrm{ml}$ of culture was vacuumfiltered onto combusted $25 \mathrm{~mm} \mathrm{GF/F} \mathrm{filters} \mathrm{(Whatmann),} \mathrm{wrapped} \mathrm{in} \mathrm{combusted} \mathrm{foil,} \mathrm{and} \mathrm{then}$ stored at $-20^{\circ} \mathrm{C}$. Before analysis, samples were dried in a warm oven, packaged in combusted foil, and sent to the Marine Science Institute Analytical Lab (Santa Barbara, CA) for CHN analysis.

Dissolved organic carbon - Samples for dissolved organic carbon (DOC) were passed through $0.2 \mu \mathrm{m}$ polycarbonate filters (GE Osmonics) into acid cleaned polypropylene tubes and stored at $20^{\circ} \mathrm{C}$. Samples were analyzed at the UC Davis Stable Isotope Facility (http://stableisotopefacility.ucdavis.edu).

Intracellular metal quota measurements - MED4ax and MIT9313ax cultures were sampled in $100 \mathrm{ml}$ volumes by vacuum-filtration onto acid-cleaned $0.2 \mu \mathrm{m}, 47 \mathrm{~mm}$, polycarbonate filters (GE Osmonics) using Teflon filter funnels and frits (Savillex). Cells were incubated on the filter with 
$3 \mathrm{ml}$ of oxalate wash for 3 minutes, according to the protocol in Tovar-Sanchez et al. 2003, and followed by a $10 \mathrm{ml}$ un-amended trace-metal clean seawater rinse. Several filter blanks were collected as well including: acid-cleaned filters, filters rinsed with un-amended trace-metal clean seawater, and filters rinsed with the oxalate solution followed by un-amended trace-metal clean seawater. Filters were then placed in acid-cleaned polypropylene tubes (Globe) and stored at $20^{\circ} \mathrm{C}$ until processing.

Filters were digested at $100^{\circ} \mathrm{C}$ on a heat block (Environmental Express) with $5 \%$ nitric acid (Seastar) for 4 hours in Teflon vials (Savillex). Dilutions of the digestion solutions were made into 5\% nitric acid to process by ICP-MS. All intracellular metal concentrations shown here were run on the ICP-MS on the same day and diluted with the same batch of $5 \%$ nitric acid. Intracellular phosphorous, iron, manganese, and cobalt concentrations were measured on an Element II Inductively-coupled plasma mass spectrometer (ICP-MS) (Thermo-Finnegan) equipped with an Aridus (Cetac Technologies) sample introduction system at the Woods Hole Oceanographic Institution ICP-MS facility. X-cones were used to improve sensitivity above standard cones and the instrument was tuned on ${ }^{115} \mathrm{In}$ and ${ }^{238} \mathrm{U}$ to set the maximum signal intensity. Sample signal intensities were converted to concentrations using P31 standard dilutions and a mixed Fe56, Mn55, and Co59 standard dilutions in 5\% nitric acid.

Because of technical difficulties measuring intracellular trace metal concentrations in all of our samples, here, we focus on trace metal concentrations in cells acclimated to $0.19 \mathrm{nM} \mathrm{Fe}$ '. See "Future Directions" section for details on improving this method.

\section{Results and Discussion}

General cell physiology

We first studied the steady-state growth rates of MED4ax and MIT9313ax at two light intensities and a range of iron concentrations. Light intensity was influential in setting the growth rates of MED4ax and MIT9313ax, as expected (Figure 1). The maximum growth rate of ironreplete MED4ax cultures at $63 \mu \mathrm{Em}^{-2} \mathrm{~s}^{-1}$ was nearly double the growth rate of iron-replete MED4ax cultures at $20 \mu \mathrm{Em}^{-2} \mathrm{~s}^{-1}$ of $0.9 \mathrm{day}^{-1}$ and $0.5 \mathrm{day}^{-1}$, respectively. The growth rate of MIT9313ax iron-replete cultures at $20 \mu \mathrm{Em}^{-2} \mathrm{~s}^{-1}$ was close to that of MED4ax at $20 \mu \mathrm{Em}^{-2} \mathrm{~s}^{-1}$ at about $0.55 \mathrm{day}^{-1}$, which is important in setting up a control for growth rate when comparing the iron physiology of the two strains (see Methods for an explanation of the choice of light intensities). The growth rates of MIT9313ax cultures were also significantly controlled by light 


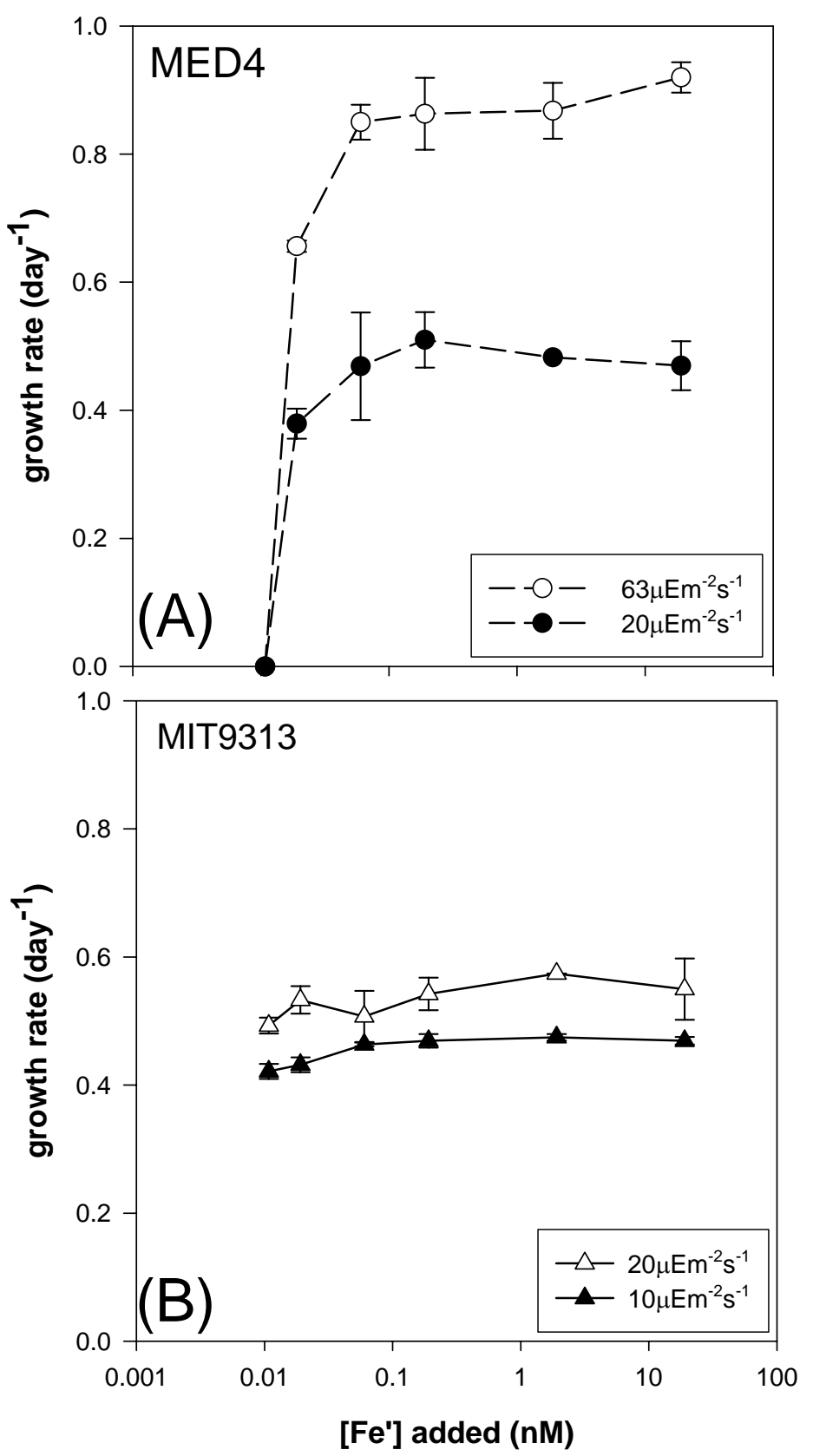

Figure 1: Steady-state growth rates over a range of iron concentrations and at two light intensities. Media from the same bottle was used for both strains at each light level for every concentration of iron. Growth rates were calculated from changes in cell density measured by flow cytometry. (A) MED4ax (B) MIT9313ax. 

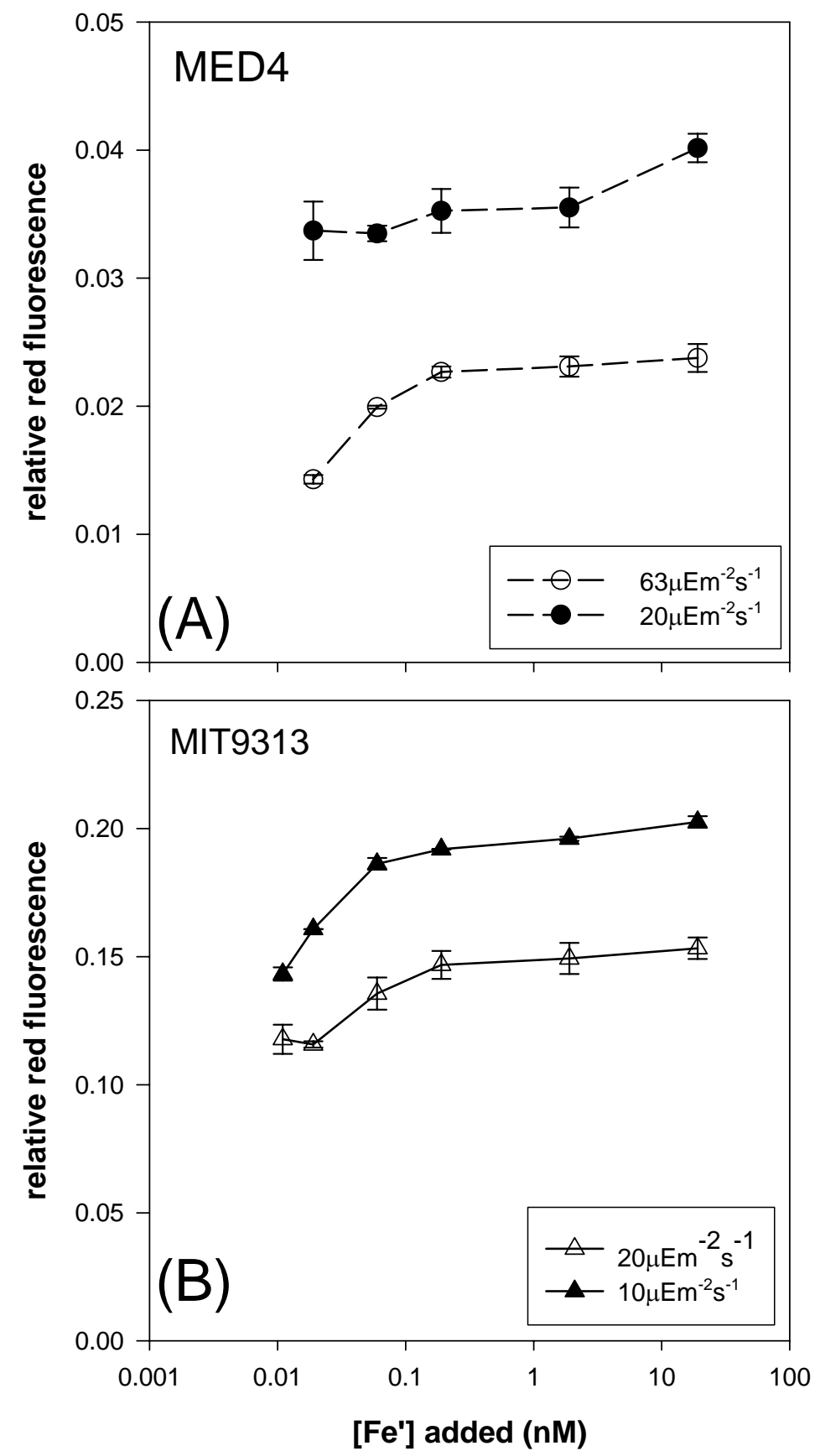

Figure 2: Red fluorescence per cell measured by flow cytometry relative to standard beads over a range of iron concentrations and at two light levels. Samples were collected during mid-log phase growth after seven transfers at constant iron concentrations (A) MED4ax. (B) MIT9313ax. 


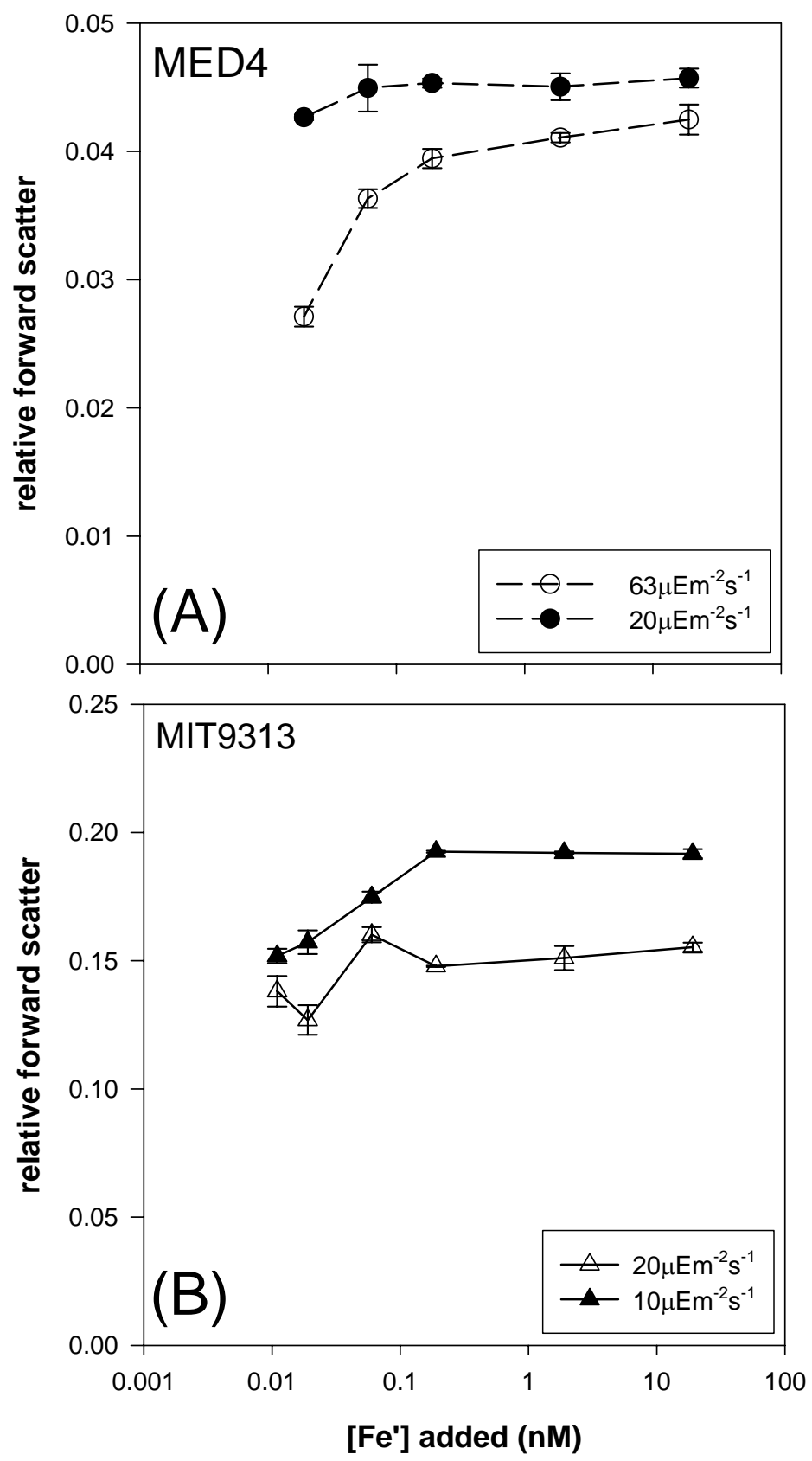

Figure 3: Forward scatter measured by flow cytometry relative to standard beads over a range of iron concentrations and at two light levels. Samples were collected during mid-log phase growth after seven transfers at constant iron concentrations (A) MED4ax. (B) MIT9313ax. 
intensity. Yet, the difference in light intensity $\left(10 \mu \mathrm{Em}^{-2} \mathrm{~s}^{-1}\right)$ was only great enough to yield a subtle reduction in growth rate of the culture acclimated to the lower light intensity of $10 \mu \mathrm{Em}^{-2} \mathrm{~s}^{-1}$ to about 0.45 day $^{-1}$ (Figure 1). These results are consistent with previous findings that MED4ax is adapted to higher light intensities and MIT9313ax is adapted to lower light intensities for optimal growth (Moore and Chisholm, 1999). Though, the actual growth rate at each light intensity for each strain is higher than in the previously published study. We hypothesize that this is due to the slightly higher temperature used here, $25^{\circ} \mathrm{C}$, compared to $24^{\circ} \mathrm{C}$ (Moore et al, 1999).

As expected, MED4ax and MIT9313ax cultures growing at the higher light intensity had less red fluorescence per cell than cultures growing at lower light intensity (Figure 2). Also, cells growing at the lower light intensity were larger than cells growing at the higher light intensity when relative forward scatter is used as a proxy for cell size as measured by flow cytometry (Figure 3). These results are consistent with the observations that cells acclimated to low light have increased red fluorescence per cell and cell size, which could serve to maximize lightharvesting efficiency. Notably, MIT9313ax is both larger and has stronger red fluorescence than its high-light adapted relative, MED4ax. This result is consistent with previous work on Prochlorococcus physiology (Moore et al, 1999).

Minimum iron requirement of MED4ax vs. MIT9313ax - Growth rates for MED4ax and MIT9313ax over a range of iron concentrations and two light intensities indicate that MIT9313ax is better adapted to survive at lower iron concentrations than MED4ax. This result is evident when we look at the steady-state growth rates of each strain over the range of iron concentrations (Figure 1) and at the fraction of the maximum growth rate for each strain and light intensity over the range of iron concentrations (Figure 4a). MED4ax is incapable of surviving at $0.01 \mathrm{nM} \mathrm{Fe}$ ' where MIT9313ax is growing near its maximum iron-replete growth rate. The range of iron concentrations used in this experiment did not extend low enough to detect the concentration of Fe' that prevents MIT9313ax growth. We estimate that MIT9313ax would become completely iron limited at a concentration of Fe' less than $0.01 \mathrm{nM}$ but greater than $0.001 \mathrm{nM}$ based on its trajectory in Figure 4a.

Examining iron stress, instead of iron limitation, as described above, shows less distinction between how iron affects MED4ax and MIT9313ax at each Fe' concentration. Here, we consider decreased red fluorescence per cell and decreased relative forward scatter as signs of iron stress. In contrast, we define iron limitation as a reduction in cellular growth rate due to lack 

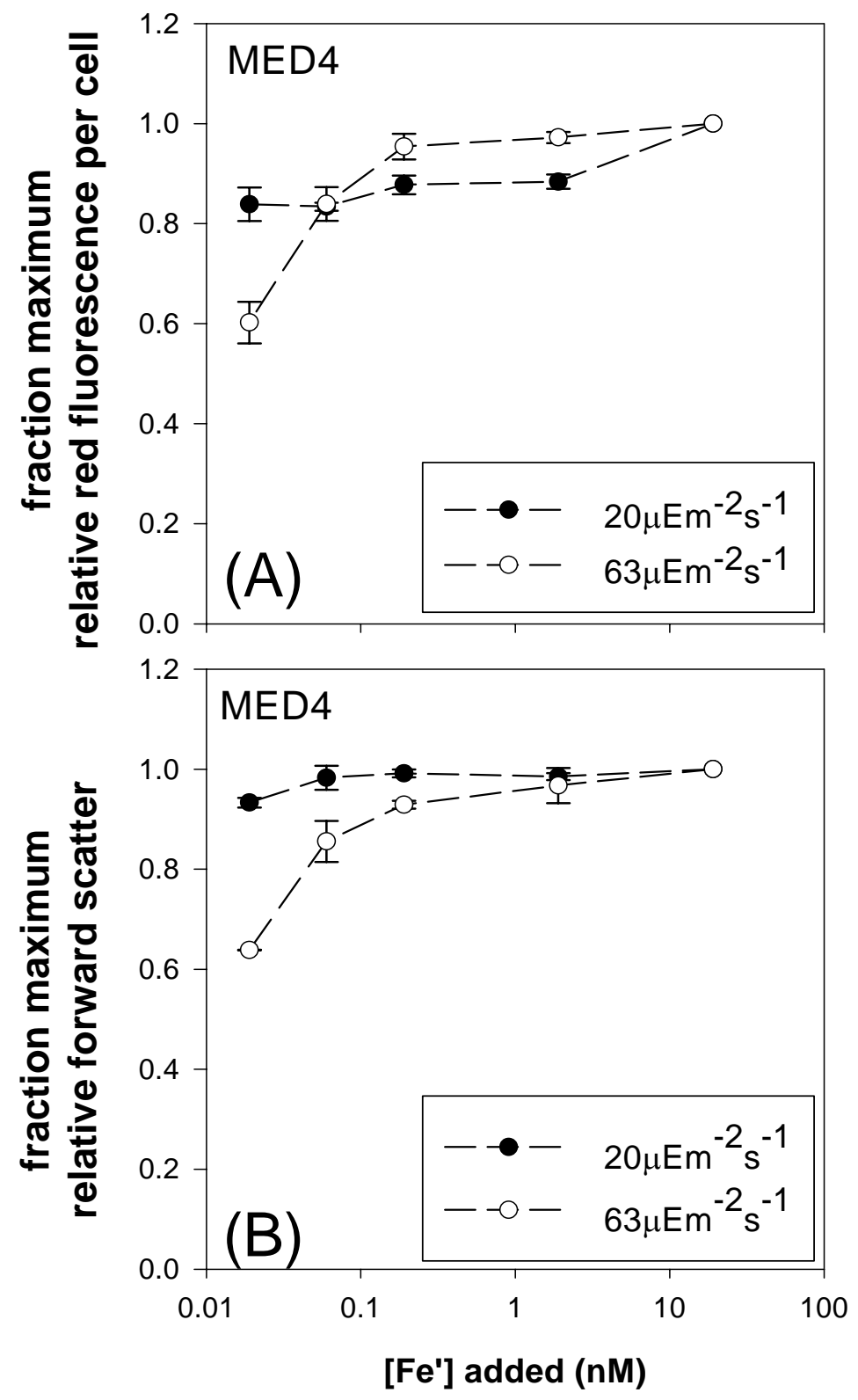

Figure 5: Study in the effect of light availability on the concentration of Fe' that induces iron stress in MED4ax. (A) Fraction of the maximum red fluorescence per cell at each light intensity and over the range of iron concentrations. (B) Fraction of the maximum forward scatter at each light intensity and over the range of iron concentrations. 

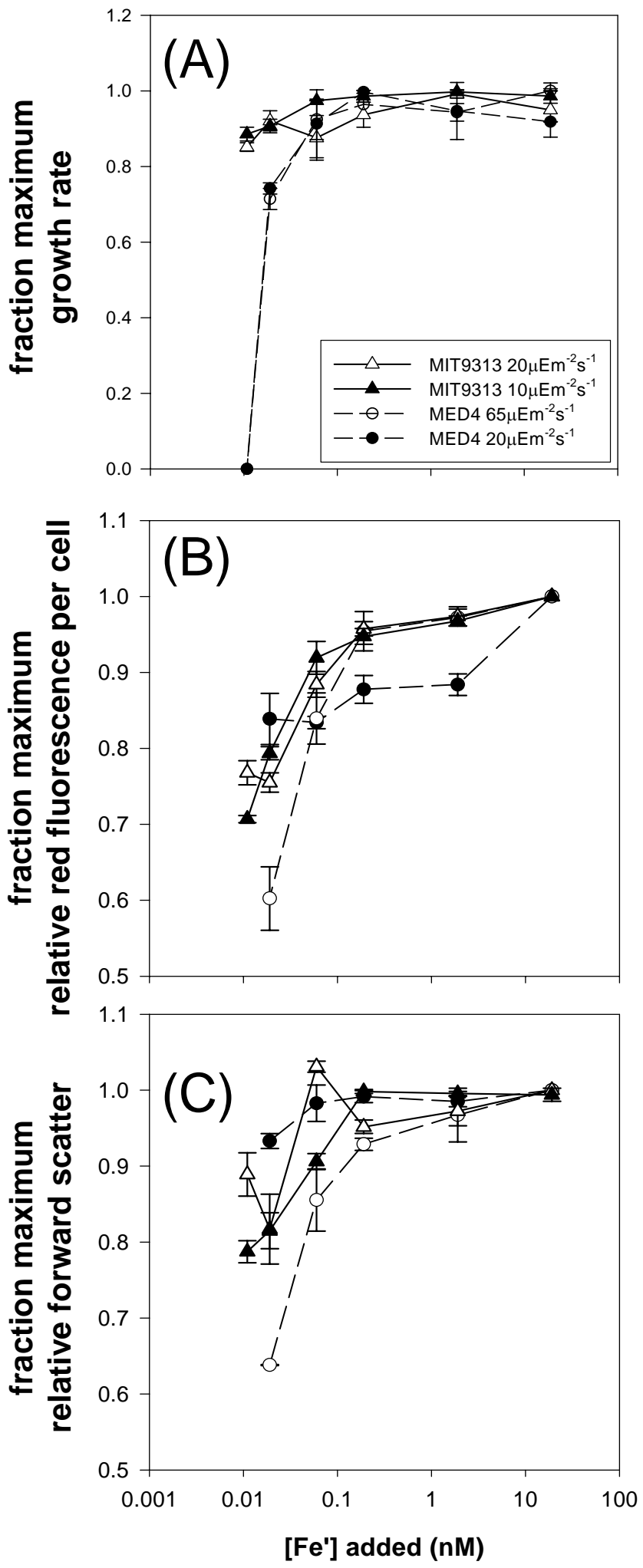

Figure 4: MED4ax compared to MIT9313ax over a range of iron concentrations. (A) Fraction of the maximum growth rates for each light intensity over the range of iron concentrations for MED4ax and MIT9313ax. (B) Fraction of the maximum red fluorescence per cell for each light level over the range of iron concentrations for MED4ax and MIT9313ax. (C) Fraction of the maximum forward scatter for each light intensity over the range of iron concentrations for MED4ax and MIT9313ax. 
of iron and it can be thought of as a more severe form of iron stress. Figure 4 (b and c) display the fraction of maximum red fluorescence per cell or forward scatter for each strain, light intensity, and iron concentration that was tested. The results do not show as striking a difference between MED4ax and MIT9313ax as does growth rate in Figure 4a. This result suggests that while there are similar intracellular consequences of iron stress in MED4ax and MIT9313ax (such as degradation of chlorophyll and increases in surface area to volume ratios), MIT9313ax is able to continue dividing despite iron stress while MED4ax is not.

MED4ax and MIT9313ax are different in several ways which could explain the ability of MIT9313ax to maintain near-maximal growth rates at lower iron concentrations than MED4ax. First, MED4ax was isolated from the Mediterranean Sea which has relatively high concentrations of trace metals compared to the Gulf-stream where MIT9313ax was isolated (Boyle et al, 1985; Sherrell and Boyle, 1988). Among diatoms and dinoflagellates, origin of isolation explains the higher iron requirement in coastal strains when compared to open-ocean isolates (Sunda and Huntsman, 1995). In Prochlorococcus, phosphorous physiology appears to be determined by origin of isolation so it would be possible that iron physiology is determined by origin of isolation as well (Martiny et al, 2006). If MIT9313ax is adapted to low iron conditions, it is likely better at acquiring iron from the environment or has a lower cellular iron requirement than MED4ax. The fact that MIT9313ax is more susceptible to copper toxicity than MED4ax suggests that the former is true (Mann et al, 2002). With a higher affinity iron acquisition system, MIT9313ax would both be better adapted to acquiring iron from the environment when it is scarce and would be more likely to mistakenly uptake copper than MED4ax. Yet, so far, there is no evidence of competitive inhibition of iron uptake by copper.

Iron physiology and light physiology are closely tied to each other and present another perspective from which to consider the ability of MIT9313ax to survive at lower iron concentrations than MED4ax. Based on their light physiologies, we hypothesized that MIT9313ax, the low-light adapted ecotype, would require more iron than the high-light adapted MED4ax due to its greater reliance on iron-demanding light-harvesting complexes for adaptation to low light. Therefore, it may require higher concentrations of iron for growth. As discussed above, our results do not support this hypothesis. Bibby et al. 2003, suggest that MIT9313ax may be more adept at increasing its light-harvesting efficiency during iron stress due to its expression of an additional antennae protein, pcbB, which forms a supercomplex with PSI during iron stress. MED4ax does not contain an iron-responsive pcb protein (Bibby et al, 2003). We hypothesize 
that in addition to possessing a higher-affinity iron-uptake system, MIT9313ax may be better suited to preserve its light-harvesting efficiency during iron stress through the expression of additional antennae proteins allowing it to persist at lower iron concentrations than MED4ax.

One hypothesis for the success of Prochlorococcus in oligotrophic regimes is that its small size increases its surface to volume ratio allowing it to acquire more nutrients and thrive with a smaller nutrient quota relative to other larger cells (Raven, 1988). Clearly, for both MED4ax and MIT9313ax, reduction of cell size accompanies acclimation to reduced iron concentrations (Figure 3). Yet, our results are contrary to the idea that cell size alone determines the minimum external iron concentration necessary for growth in Prochlorococcus. At $20 \mu \mathrm{Em}^{-2} \mathrm{~s}^{-}$ ${ }^{1}$, MIT9313ax is about three times larger than MED4ax (Figure 3), yet is able to continue growing at iron concentrations that completely inhibit MED4ax (Figure 3 and 4). We hypothesize that among Prochlorococcus isolates diversity in iron physiology is due to differences at the molecular level that control iron acquisition and storage as well as light-harvesting efficiency.

Influence of light availability on iron physiology - The concentration of iron that induces growth rate limitation in MED4ax and MIT9313ax is independent of light availability. This result is evident when we look at the fraction of the maximum growth rate of cultures at a common light intensity over a range of iron concentrations (Figure 4a). This result contrasts with our hypothesis that the iron requirement of low-light acclimated cells would be greater due to the involvement of iron in photosynthetic electron transport (Raven, 1988). Our results are consistent however with the results of Sunda and Huntsman (1997) that performed similar light availability vs. iron availability experiments with diatoms and dinoflagellates. Sunda and Huntsman (1997) measured higher Fe: $\mathrm{C}$ ratios in low-light acclimated cultures and thus hypothesized, like us, that cells acclimated to low light intensity would suffer decreased growth rates at iron concentrations higher than their high-light acclimated counterparts. As we observed, their results showed that the concentration of iron that induced growth rate limitation was independent of irradiance, which is a result contrary to their hypothesis. Sunda and Huntsman (1997) explain this unexpected result through the connections between specific growth rates, intracellular Fe: $\mathrm{C}$ ratios, and iron uptake rates normalized to carbon. They suggest that cells acquire iron at the maximum rate allowed by physics and chemistry, which is independent of light intensity. Therefore, cells that divide less frequently due to light limitation will accumulate more iron relative to carbon than cells that are dividing more frequently. The difference in growth rate and constant iron uptake rate results in an 


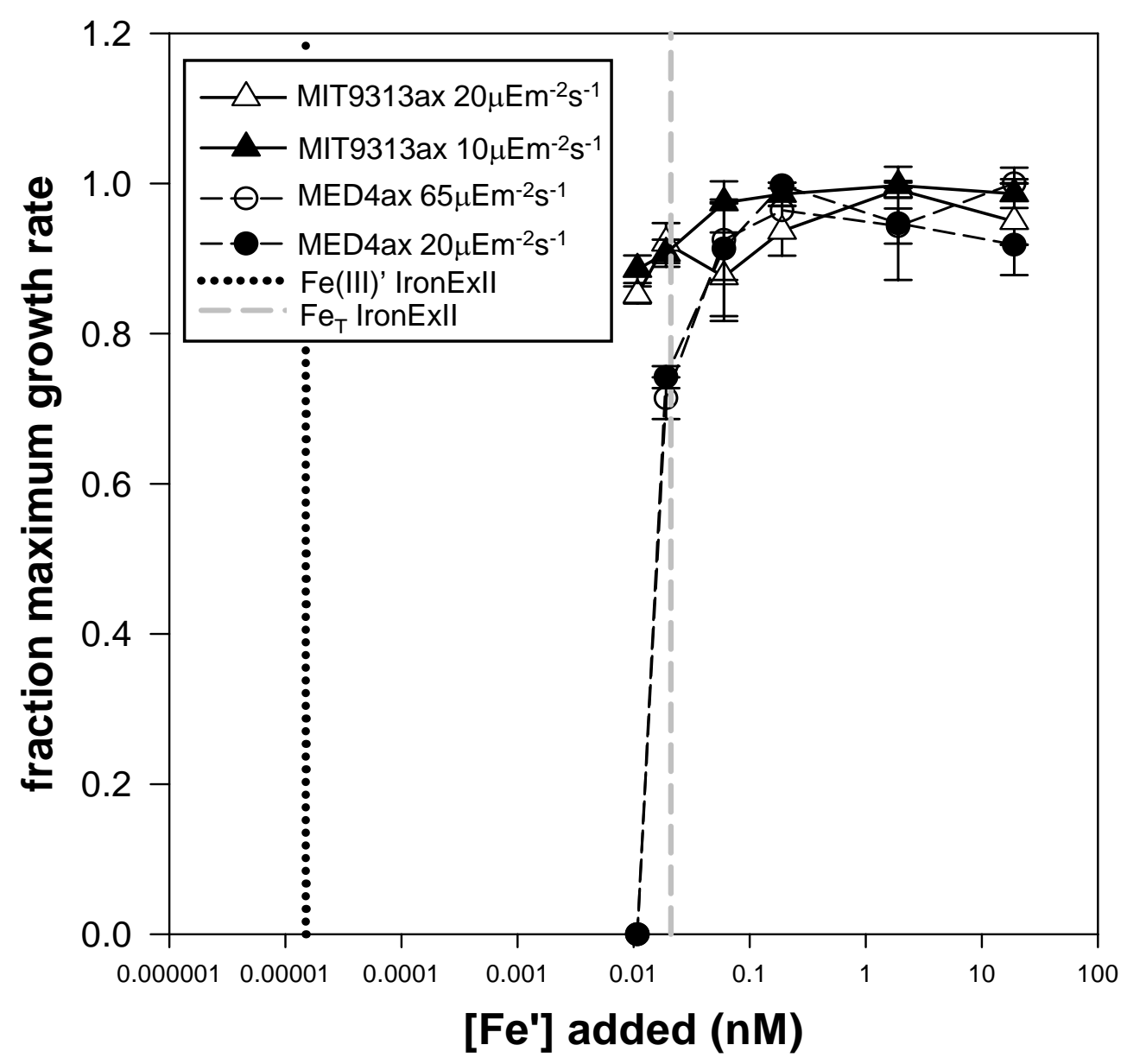

Figure 6: Comparison of iron concentrations at IronExII that support half-maximum growth rates in natural Prochlorococcus populations compared to growth rates in culture. Prochlorococcus cultured isolates are limited at much higher Fe' concentrations in defined media than in the natural environment. 
accumulation of iron in the light-limited cells that allows synthesis of additional photosystem complexes required for low-light adaptation (Sunda and Huntsman, 1995, 1997).

The relationship between light and iron requirement appears different when we consider how acclimation to a particular light intensity influences the concentration of iron that causes stress rather than limitation. In MED4ax, cultures acclimated to high light intensity experience iron stress at higher iron concentrations than cells acclimated to low light intensity. This effect is visible when we look at the fraction of maximum red fluorescence and fraction of maximum forward scatter for each light intensity over the range of iron concentrations tested (Figure 5). We used MED4ax to illustrate this effect (Figure 5) as the light intensities used for MED4ax growth produced significant differences in growth rate whereas the light intensities used to culture MIT9313ax produced only mild growth rate differences (Figure 1). Simply, our results demonstrate that at a given iron concentration, MED4ax cells acclimated to high-light would be more iron-stressed than cells acclimated to low-light relative to iron-replete cultures at both light intensities. Possibly, this effect could be related to the higher growth rate of MED4ax acclimated to high light.

Minimum iron concentration requirement in the field vs. the lab - We compared the steady-state growth rates of MED4ax and MIT9313ax to Prochlorococcus growth rates measured outside the iron-fertilization patch at IronExII (Mann et al, 2000). The comparison shows that the concentration of Fe' that allows Prochlorococcus to grow at half its maximum growth rate in the Equatorial Pacific is orders of magnitude less than the concentration of Fe' that permits MED4ax and MIT9313ax (by continuation of its trajectory) to grow at half the maximum rate in culture (Figure 6). Interestingly, the concentration of total $\mathrm{Fe}\left(\mathrm{Fe}_{\mathrm{T}}\right)$ in the field that allows half-maximum growth rates is more comparable to the concentrations of available iron (Fe' in an EDTA buffered medium) in culture.

We can imagine three possible explanations of why field concentrations of $\mathrm{Fe}_{\mathrm{T}}$ are more relevant than Fe' for predicting the amount of iron that supports Prochlorococcus growth in culture. First, MED4ax and MIT9313ax were not isolated from the Equatorial Pacific and therefore may not be adapted for survival at the low iron concentrations prevalent in the Equatorial Pacific. This idea is supported among Synechococcus strains as a clone isolated in the Pacific, A2169, had higher cell-yield at low iron concentrations than other Atlantic-derived strains (Brand, 1991). We have several Prochlorococcus strains of Pacific origin that would be 

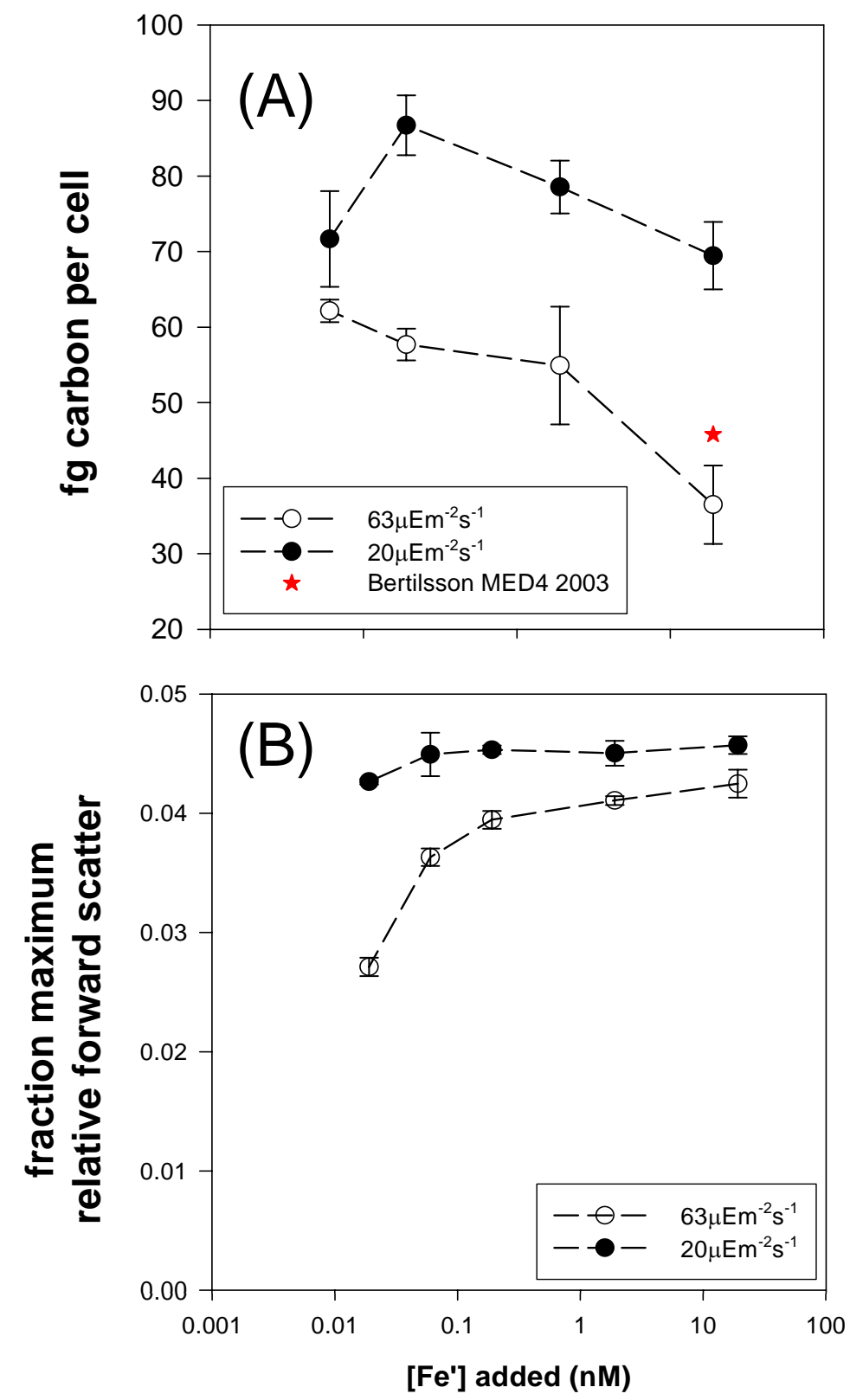

Figure 7: Carbon per cell in MED4ax compared to relative forward scatter as a proxy for cell size over a range of iron concentrations and at two light levels. (A) Carbon content per cell increases with decreasing iron concentration in MED4ax acclimated to $20 \mu \mathrm{Em}^{-2} \mathrm{~s}^{-1}$ and $63 \mu \mathrm{Em}^{-2} \mathrm{~s}^{-1}$. Carbon content measured in this study is comparable to values measured for MED4ax in 2003 published in Bertilsson et al. 2003. (B) Relative forward scatter per cell decreases with decreasing iron concentrations for MED4ax and suggests that the increase in carbon content per cell is not due to increasing cell size. 

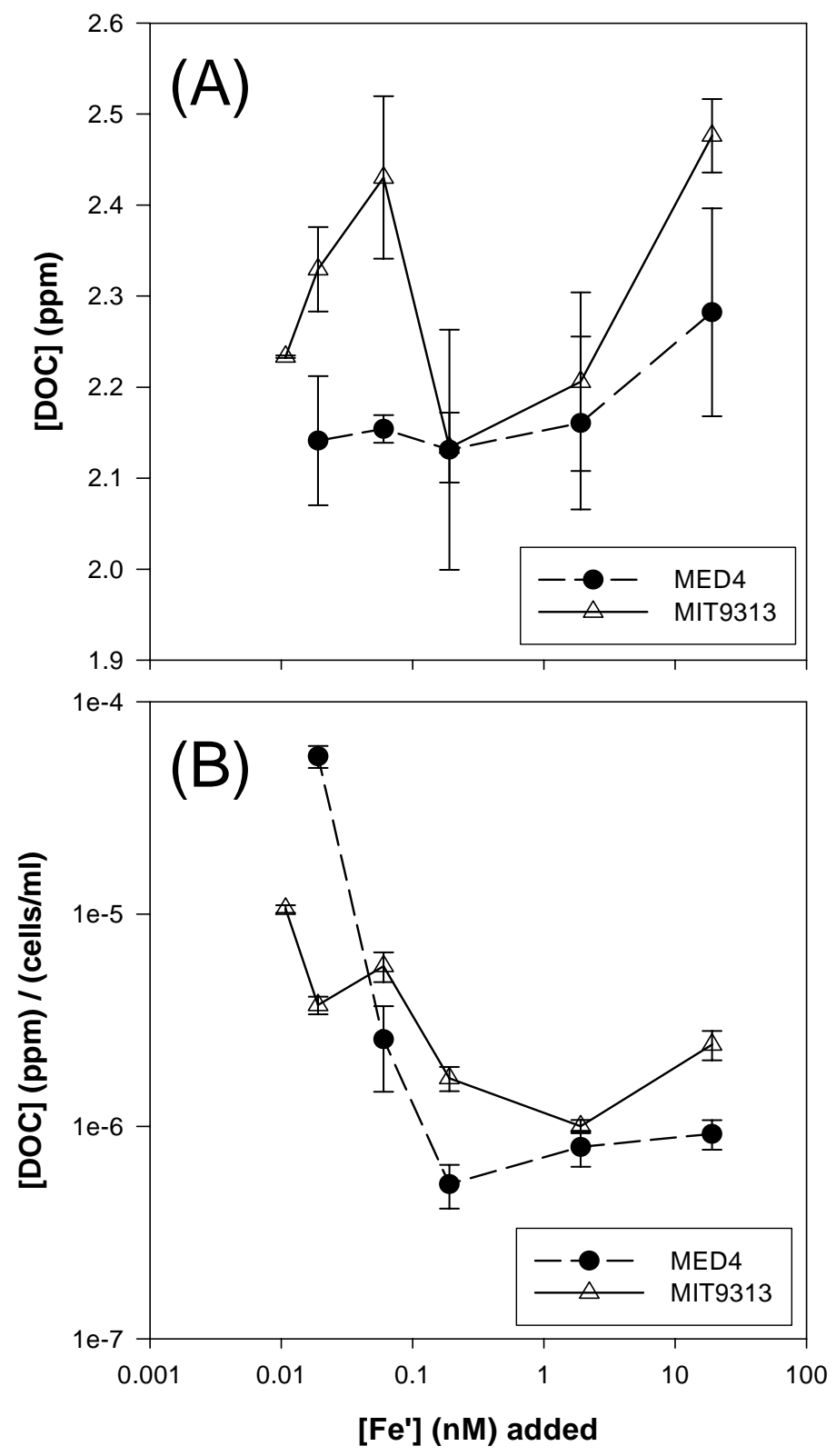

Figure 8: Dissolved organic carbon (DOC) concentrations measured in MED4ax and MIT9313ax over a range of iron concentrations and at $20 \mu \mathrm{Em}^{-2} \mathrm{~s}^{-1}$. (A) Total DOC concentrations in 100ml culture from each culture. (B) DOC concentrations normalized to cell density of the source culture for DOC samples. At high iron concentrations, MIT9313ax cultures contain more DOC than MED4ax cultures. In both strains, decreasing iron concentrations are associated with an increase in DOC concentration normalized to cell concentration. 
interesting to test against MED4ax and MIT9313ax over a range of iron concentrations to address this possibility.

The second explanation addresses the fact that the Prochlorococcus strains studied here have been cultured in iron-replete media for over a decade and have possibly evolved higher iron requirements or favored the retention of low-affinity iron acquisition systems in their genomes to match the constant high iron concentrations in the typical PRO99 medium. Yet notably, we have not seen changes in other aspects of Prochlorococcus cellular physiology in the laboratory. For example, despite many years in culture under a limited range of light availabilities, Prochlorococcus ecotypes have maintained their distinct light physiologies (Coe and Moore, unpublished).

The third explanation examines the idea that iron availability could be different between the EDTA-buffered medium of the culture experiments, where we typically expect that only Fe' is available (Hudson et al, 1990; Shaked et al, 2005), and the open ocean where iron speciation is more complex with the existence of many different types and sources of iron-binding ligands (Hunter et al, 2007; Rue and Bruland, 1997). We hypothesize that in the field, $\mathrm{Fe}_{\mathrm{T}}$ concentrations are more important in influencing Prochlorococcus ecology than in culture because of the availability of naturally-occurring iron-ligand complexes for uptake.

Cell size, carbon per cell, and dissolved organic carbon in MED4ax - The amount of carbon per cell increased with decreasing Fe' concentrations (Figure 7). Interestingly, this result conflicts with the decrease of relative forward scatter at low-iron concentrations on the assumption that both carbon per cell and relative forward scatter serve as proxies for cell size (Figure 7). We are confident in our carbon measurements as the amount of carbon per cell of MED4ax cultures growing in iron-replete conditions was similar to the carbon per cell of MED4ax that was previously measured (Figure 7) (Bertilsson et al, 2003). Based on this result, we would hypothesize that either increased storage of carbon or production of surface-associated extracellular carbon compounds accompanies iron limitation and iron stress in Prochlorococcus. We hope that future work will examine this question more closely.

Concentrations of dissolved organic carbon (DOC) were also measured for MED4ax and MIT9313ax cultures grown at $20 \mu \mathrm{Em}^{-2} \mathrm{~s}^{-1}$ over the range of iron concentrations (Figure 8) with the goal of detecting the secretion of any extracellular carbon-based compounds that are produced in response to iron-limitation and iron-stress. Notably, when normalized to cell concentration, 
DOC concentrations increase with decreasing iron concentrations in both MED4ax and MIT9313ax (Figure 8). The increasing DOC concentrations are consistent with the increase in carbon per cell (Figure 7) during iron starvation. More work is needed to bound this effect and understand its consequences for Prochlorococcus during conditions of low iron availability.

Intracellular metal quotas at a fixed iron concentration - Intracellular concentrations of P, Fe, Mn, and Co were measured for MED4ax and MIT9313ax at two different light levels during steady-state growth at a fixed Fe' concentration of $0.19 \mathrm{nM}$ to assess the influence of light on intracellular trace-metal quotas in distinct Prochlorococcus ecotypes. We observe that MED4ax and MIT9313ax cultures growing at higher light intensities contain less iron per cell and less Fe: P than cultures growing at lower light intensities (Figure 9a and Figure 10). This result supports the work of Sunda and Huntsman (1997) showing that decreased light availability induced higher Fe: $\mathrm{C}$ ratios in several species of diatoms and dinoflagellates. These results are explained by the assumption that cells acquire iron at the maximum rates permitted by physics and chemistry and therefore cells acclimated to low light conditions cannot acquire iron at a different rate than the same species acclimated to high light conditions. Light limitation forces cells to divide more slowly, which in turn allows iron to accumulate in the cell and allow acclimation to low light conditions by increased production of iron-requiring light-harvesting apparatuses (Sunda and Huntsman, 1995, 1997). Our results for Fe: $\mathrm{P}$ and Fe per cell are consistent with this argument.

Cellular Mn concentrations (Mn: P and Mn per cell) are higher in low-light acclimated MIT9313ax cells compared to high-light acclimated MIT9313ax cells and appear to support the above mechanism of raising intracellular metal concentrations during light limitation by accumulation due to lower growth rates (Figure 9b and 10). Interestingly, in MED4ax, cellular Mn concentrations do not appear to be as easily influenced by light availability though the difference in growth rates of MED4ax cultures at the two different light intensities is much greater than between MIT9313ax cultures. This difference between MED4ax and MIT9313ax in terms of the influence of light availability on Mn concentrations in the cell could suggest different relationships between Mn metabolism and light availability within the two Prochlorococcus ecotypes. The effects of light availability on cellular Co concentrations, per cell and relative to intracellular P, are inconclusive due to the high error in the measurements (Figure 9c and 10). At the same light intensity of $20 \mu \mathrm{Em}^{-2} \mathrm{~s}^{-1}$, we determined that MIT9313ax has more Fe per cell (Figure 9a) and more Fe: P than MED4ax (Figure 10). Because neither culture is growth- 
rate limited at this iron concentration, we cannot infer that MIT9313ax has a higher iron requirement than MED4ax due to the possibility of luxury uptake. MIT9313ax cells simply contain more iron than MED4ax cells at this concentration of Fe'. The larger cell size of MIT9313ax could explain the elevated concentration of Fe per cell compared to MED4ax. Or, the higher concentration of iron-requiring light harvesting complexes in MIT9313ax could account for the greater concentration of Fe in MIT9313ax than MED4ax. Interestingly, both MED4ax and MIT9313ax cultures at $20 \mu \mathrm{Em}^{-2} \mathrm{~s}^{-1}$ have the same growth rate (Figure 4a). If iron uptake rates were the same for both strains, we would expect the cells to contain the same amount of iron per cell (Sunda and Huntsman, 1997). Because MIT9313ax contains more iron per cell than MED4ax (Figure 9a), we hypothesize that MIT9313ax acquires iron at a faster rate than MED4ax at this iron concentration and light intensity through the expression of more iron uptake systems or the expression of a high affinity iron uptake system not present in MED4ax.

MIT9313ax contains similar Mn:P and Mn per cell as MED4ax at the same light intensity (Figure 9b and 10). Strikingly, at the same metal concentrations and light intensity, MED4ax contains more Co:P and Co per cell than MIT9313ax (Figure 9c and 10). Previous work shows an absolute Co requirement in MED4ax but the cobalt requirement of MIT9313ax has not been examined (Saito et al, 2002). We hypothesize that the metabolism of MED4ax relies more heavily on Co than the metabolism of MIT9313ax. By testing the growth rates and cellular cobalt quotas of both strains over a range of growth-rate limiting cobalt concentrations, we could test their relative cobalt requirements.

For both MED4ax and MIT9313ax, the relative intracellular concentrations of Fe, Mn, and Co reflect the concentrations of these metals that are present in the PRO99-TM media (Table 1). In both strains, Fe is the most abundant next to $\mathrm{Mn}$, and finally Co (Figure 9 and 10). Yet interestingly, at the fixed media concentrations of Fe, Mn, and Co, MED4ax accumulates more Mn and Co relative to Fe than does MIT9313ax at any of the light intensities that were tested (Figure 11). MED4ax also contains more Co relative to Mn than MIT9313ax despite the same concentrations of these nutrients in the media. It will be very interesting to test the relative reliance of MED4ax and MIT9313ax on these elements under starvation conditions in future experiments. 


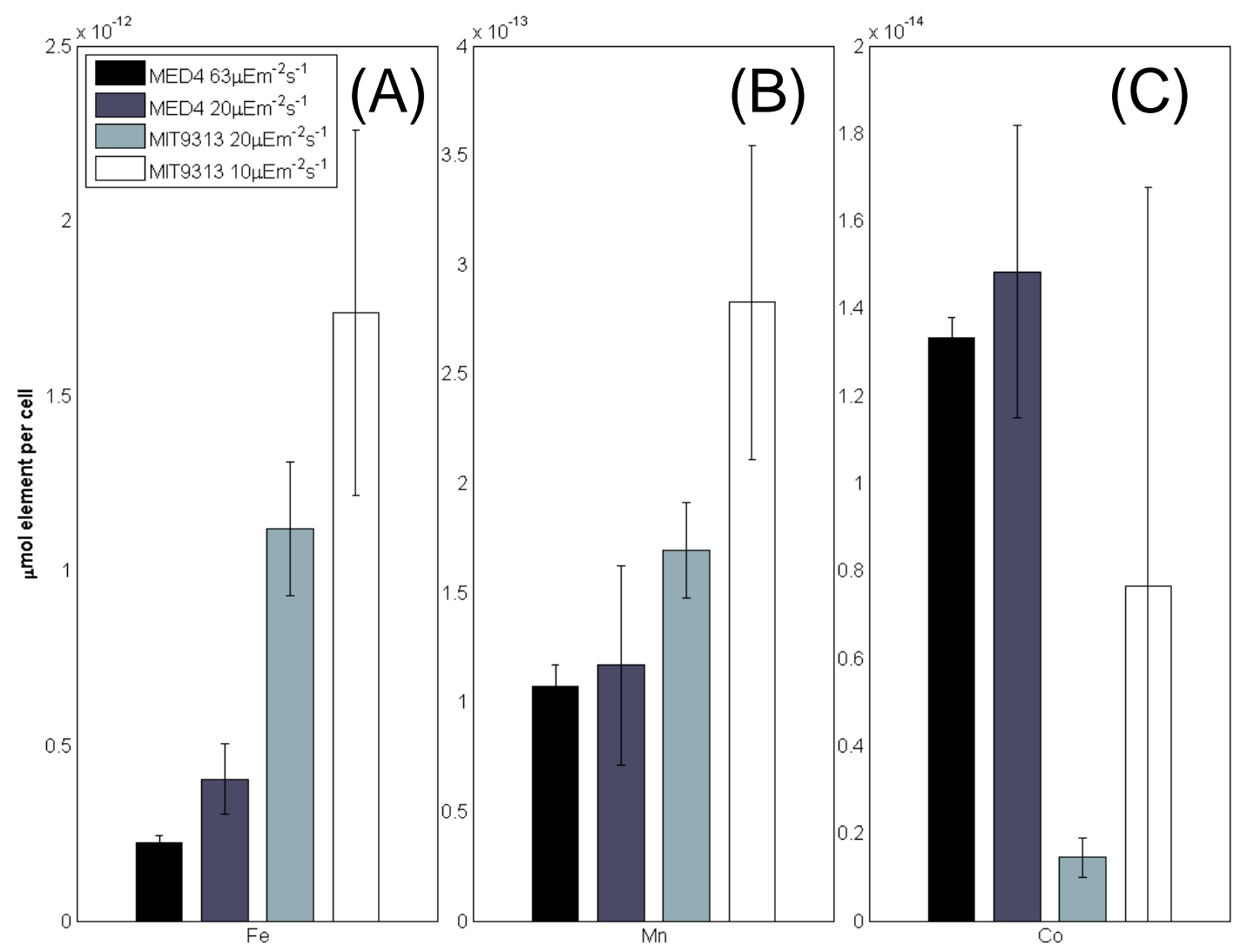

Figure 9: Intracellular metal concentrations in Prochlorococcus MED4ax and MIT9313ax at 0.19nM Fe' and at two light intensities for each strain. (A) Fe $\mu$ mol per cell is higher in low light acclimated cultures and higher in MIT9313ax compared to MED4ax at a light intensity of $20 \mu \mathrm{Em}^{-2} \mathrm{~s}^{-1}$ (B) $\mathrm{Mn} \mu \mathrm{mol}$ per cell is comparable for MED4ax and MIT9313ax at 20 $\mathrm{Em}^{-2} \mathrm{~s}^{-1}$. In MIT9313ax, low-light is associated with a higher Mn content though light availability does not significantly effect Mn per cell in MED4ax. (C) Co $\mu$ mol per cell is higher in MED4ax than MIT9313ax at $20 \mu E^{-2} s^{-1}$. It is unclear if Co quota per cell is dependent on light intensity due to high error in the measurements. 


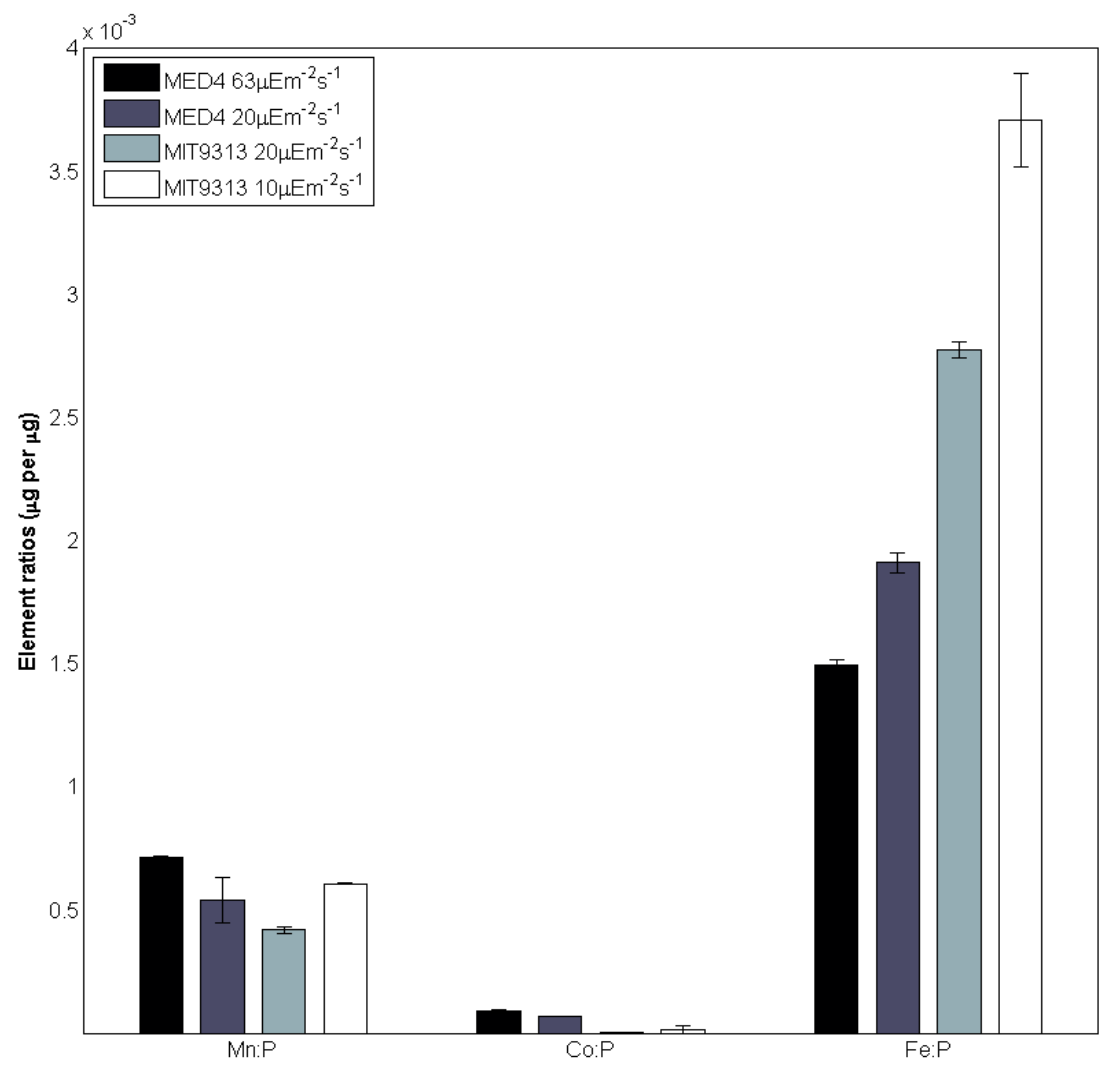

Figure 10: Intracellular metal concentrations in Prochlorococcus MED4ax and MIT9313ax at 0.19nM Fe' and at two light intensities for each strain normalized to intracellular $\mathrm{P}$ concentrations. Both strains have more Fe relative to $\mathrm{P}$ than $\mathrm{Mn}$ relative to $\mathrm{P}$. Also, both strains have more $\mathrm{Mn}$ relative to $\mathrm{P}$ than Co relative to $\mathrm{P}$ which reflects the ratio of trace metal concentrations in the PRO99-TM media. Fe:P is influenced by light availability in MED4ax and MIT9313ax while the influence of light availability on Mn:P and Co:P is not as clear in both strains. 


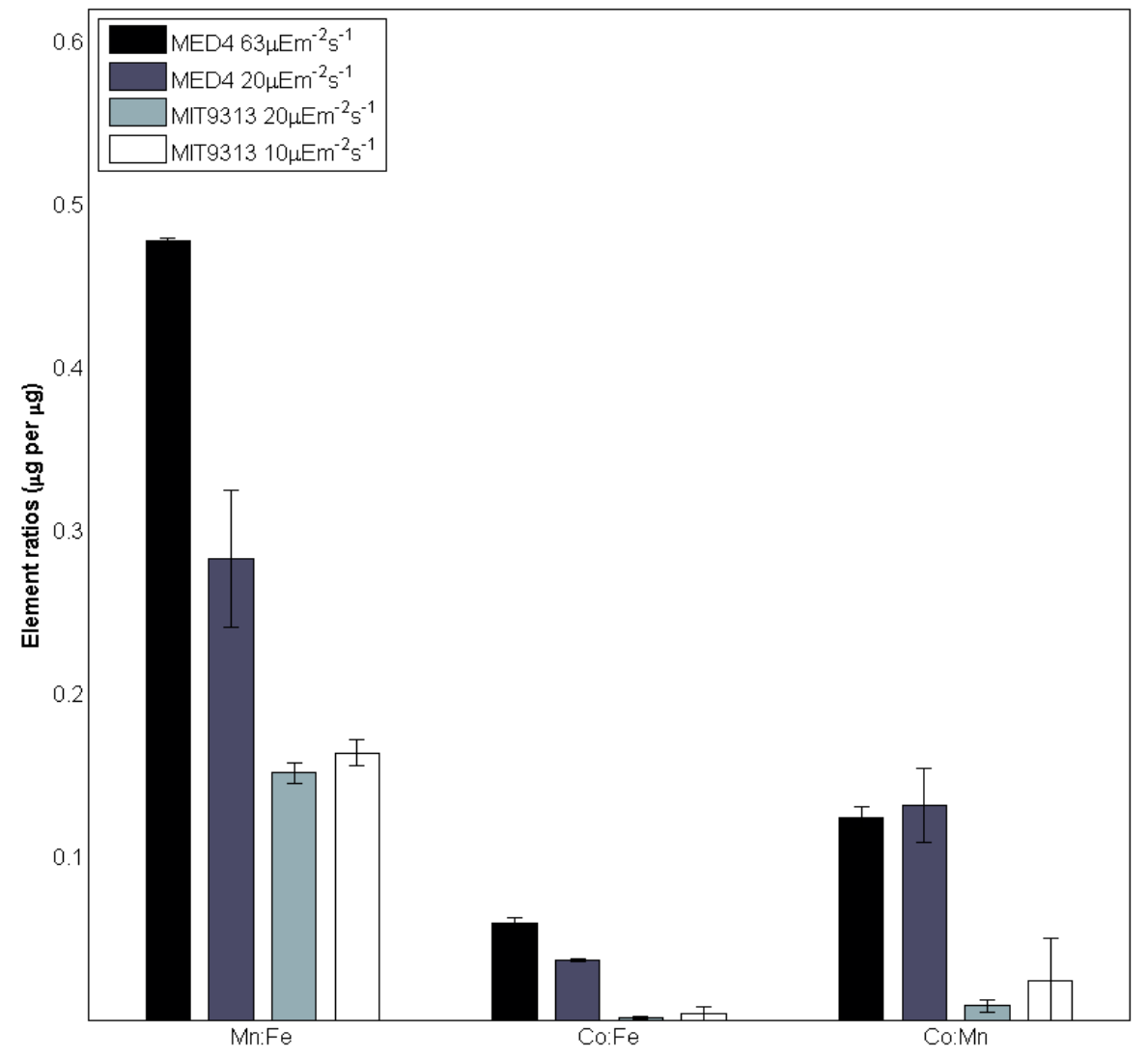

Figure 11: Intracellular trace metal ratios of $\mathrm{Fe}, \mathrm{Co}$, and $\mathrm{Mn}$ in MED4ax and MIT9313ax at 0.19nM Fe' and a two different light levels for each strain. MED4ax contains higher ratios of Mn:Fe, Co:Fe, and Co:Mn than MIT9313ax given the same media composition. 


\section{Conclusions}

Here, we have measured the growth rates, cell physiology, and intracellular trace metal quotas of two Prochlorococcus strains, MED4ax and MIT9313ax, over a range of iron concentrations and at two light intensities in order to improve our understanding of Prochlorococcus iron physiology. We found that at the same light intensity MIT9313ax is better equipped to survive at low iron concentrations than MED4ax. Also, MIT9313ax contains higher Fe: $\mathrm{P}$ ratios and higher concentrations of Fe per cell than MED4ax at a fixed concentration of iron. Interestingly, MED4ax and MIT9313ax experience iron stress at similar Fe' concentrations which presents a complex picture of how the cells cope with iron stress and iron limitation. In both strains, iron limitation of growth rate is independent of light intensity yet iron stress occurs at higher iron concentrations in MED4ax cultures acclimated to higher light intensities. Interestingly, MED4ax contains higher ratios of Co: Fe and Mn: Fe than MIT9313ax at fixed concentrations of nutrients in the media and at the same light intensity. This result inspires future work on the relative requirements of Mn and Co between MED4ax and MIT9313ax Prochlorococcus ecotypes and the iron physiologies of other Prochlorococcus isolates.

\section{Future Directions}

Prochlorococcus Fe: C ratio - At the time of this writing we are still not confident in an Fe:C ratio for Prochlorococcus. Accurately measuring Prochlorococcus Fe:C ratios in culture and in the field would be instrumental in informing global ecosystem models. We suggest a similar approach to the one followed here for measuring Fe:C in Prochlorococcus with a few improvements in the method used. In particular, we will improve our method of sampling for quotas and preparation of samples for analysis by ICP-MS. We found that in choosing only one dilution for each sample we were forced to estimate the trace metal concentrations in the cells and in some cases diluted the samples to the point where they were too close to the blank for robust measurements. Therefore, following acid-digestion, we recommend serial dilutions of samples in triplicate for analysis by ICP-MS so that at least one set of dilutions will be measured well above the digestion blank and well below the signal saturation point of the machine. In addition, we recommend storing un-diluted aliquots of digested samples in trace-metal clean Teflon vials in case the ICP-MS is not running reliably on analysis day or replicates are lost or contaminated during processing. 
Measuring Prochlorococcus Fe:C ratios in culture for comparison to the $\mathrm{Fe}: \mathrm{C}$ ratios of other marine microbes in culture would be a very useful component of marine biogeochemical models, yet, measurements of Prochlorococcus Fe:C ratios directly from the field would be much more environmentally relevant. While we did not attempt to measure Prochlorococcus-specific $\mathrm{Fe}: \mathrm{C}$ ratios in the field in this study, we believe it may be possible through cell-sorting. We foresee two major challenges in attempting to measure Prochlorococcus-specific $\mathrm{Fe}: \mathrm{C}$ ratios in the field. First, given the extremely low concentrations of iron in the field, a large number of cells will be necessary to measure low intracellular concentrations. Obtaining a large population of pure Prochlorococcus will require sorting large volumes of seawater which requires time and access to a large-volume sample of oligotrophic seawater from the euphotic zone. Second, we believe it would be a major challenge to sort cells under trace metal clean conditions. Rinsing sorted cells that have been filtered onto a membrane with an oxalate rinse could serve to remove contamination introduced during the sorting process. Alternatively, prior to sorting, the sample could be incubated with $\mathrm{Fe}^{59}$ or $\mathrm{Fe}^{57}$ to replace the naturally occurring $\mathrm{Fe}^{56}$, which is a step that could reduce issues associated with contamination during sorting. Though this project would be challenging for many reasons, it could yield environmentally relevant $\mathrm{Fe}: \mathrm{C}$ ratios for Prochlorococcus and other small auto-fluorescent cells. Many comparative questions could be asked for example, do Prochlorococcus acclimated to low-light deep in the euphotic zone have a higher Fe:C than cells acclimated to the high-light of surface waters? Or, do Prochlorococcus and Synechococcus populations from the same sample have distinguishable $\mathrm{Fe}$ : $\mathrm{C}$ ratios?

Diversity of iron physiology among Prochlorococcus isolates - Another interesting area of future work would be in assessing the iron physiology of many different Prochlorococcus isolates. Here, we have focused on the iron physiology of MED4ax and MIT9313ax because these were the axenic strains available at the time of our study. Yet, the differences between these two strains are numerous so we cannot pinpoint the reason for their distinct iron physiologies. For example, as discussed above, the two strains were isolated from different environments with different iron regimes, are adapted to different light regimes, are genetically distinct, and are of different cell size. By assessing the iron physiology of all twelve sequenced Prochlorococcus isolates we may gain insight into whether iron physiology is determined by phylogeny, origin of isolation, light physiology, or other aspects of cellular metabolism. 
A comparison between the many different Prochlorococcus strains would require that all strains are axenic. Currently, we are developing methods to render any Prochlorococcus strain axenic through flow sorting. With new axenic strains available, each strain could be grown to steady-state over a range of iron concentrations and at two different light levels to control for its optimal light intensity. Furthermore, the physiological differences between Prochlorococcus isolates could be examined in the context of the genome of each strain, which could yield important information on the iron acquisition strategies and iron-requiring proteins that play a role in determining a cell's iron physiology. We are most interested in testing the iron physiology of MIT9515, which is a high-light adapted ecotype isolated from the Equatorial Pacific. If MED4 and MIT9313 cannot grow in the laboratory at the Fe' concentrations that support Prochlorococcus growth in the field because they are not adapted to low-iron regimes, then MIT9515 should be capable of growth at very low Fe' concentrations.

Temperature vs. light in limiting growth rate and inducing high $\mathrm{Fe}: \mathrm{C}$ - Our results indicate that Prochlorococcus cells acclimated to lower light intensity have relatively more iron per cell than cells acclimated to higher light intensity. The studies of Sunda and Huntsman (1997) demonstrate a high cellular Fe: $\mathrm{C}$ ratio in diatoms and dinoflagellates acclimated to low light compared to high light. Sunda and Huntsman (1997) suggest that the higher Fe:C ratio in low light acclimated cells could be accounted for by the fact that their iron uptake rates are the same as high light adapted cells but that growth rate is reduced at lower light intensities leading to an accumulation of $\mathrm{Fe}$ in each cell. This explanation can easily be turned into the testable hypothesis that cultures with lower growth rates have higher Fe:C. We suggest an experiment where Fe: $\mathrm{C}$ ratios of Prochlorococcus are measured when growth rates are limited both by light intensity and temperature and would expect $\mathrm{Fe}: \mathrm{C}$ to be lower in cultures growing a slower rates. Another experiment that could test how light influences $\mathrm{Fe}: \mathrm{C}$ would be by growing two cultures are different light intensities and use temperature to set the growth rates of both cultures as equal. In this case, if growth rate alone sets the Fe: $\mathrm{C}$ of the cell, both cultures should have the Fe:C ratios despite being grown under different light intensities.

\section{Acknowledgements}

A.W.T. is grateful to Tyler Goepfert for his technical assistance in the laboratory of M.S. at the Woods Hole Oceanographic Institution. Thanks to Dawn Moran for packaging DOC samples and 
sending them out for analysis. Thanks to Erin Bertrand for assistance in monitoring cultures. John Waterbury kindly allowed use of his walk-in light incubator at WHOI. Dave Schneider at the WHOI ICP-MS facility deserves thanks for running the Element II ICP-MS and technical assistance related to the ICP-MS sample processing. This work was supported by ONR grant to

M.S. and grants through the Moore Foundation and DOE to S.W.C.

\section{References}

Aumont O, Maier-Reimer E, Blain S, Monfray P (2003) An ecosystem model of the global ocean including $\mathrm{Fe}, \mathrm{Si}, \mathrm{P}$ colimitations. Global Biogeochemical Cycles 17.

Bertilsson S, Berglund O, Karl DM, Chisholm SW (2003) Elemental composition of marine Prochlorococcus and Synechococcus: Implication for the ecological stoichiometry of the sea. Limnology and Oceanography 48: 1721-1731.

Bibby TS, Mary I, Nield J, Partensky F, Barber J (2003) Low-light-adapted Prochlorococcus species possess specific antennae for each photosystem. Nature 424: 1051-1054.

Boyle EA, Chapnick SD, Bai XX, Spivack A (1985) Trace metal enrichments in the Mediterranean Sea. Earth and Planetary Science Letters 74: 405-419.

Brand LE (1991) Minimum iron requirements of marine phytoplankton and the implications for the biogeochemical control of new production. Limnology and Oceanography 36: 1756-1771.

Follows MJ, Dutkiewicz S, Grant S, Chisholm SW (2007) Emergent Biogeography of Microbial Communities in a Model Ocean. Science 315: 1843-1846.

Hudson RJM (1998) Which aqueous species control the rates of trace metal uptake by aquatic biota? Observations and predictions of non-equilibrium effects. Science of The Total Environment 219: 95-115.

Hudson RJM, Morel FMM (1990) Iron Transport in Marine Phytoplankton: Kinetics of Cellular and Medium Coordination Reactions. Limnology and Oceanography 35: 1002-1020.

Hunter KA, Boyd PW (2007) Iron-binding ligands and their role in the ocean biogeochemistry of iron. Environmental Chemistry 4: 221-232.

Katoh H, Hagino N, Grossman AR, Ogawa T (2001) Genes Essential to Iron Transport in the Cyanobacterium Synechocystis sp. Strain PCC 6803. Journal of Bacteriology 183: 2779-2784.

Keller MD, Bellows WK, Guillard RL (1988) Microwave treatment for sterilization of phytoplankton culture media. Journal of Experimental Marine Biology 117: 279-283.

Kettler GC, Martiny AC, Huang K, Zucker J, Coleman ML, Rodrigue S, Chen F, Lapidus A, Ferriera S, Johnson J, Steglich C, Church GM, Richardson P, Chisholm SW (2007) Patterns and Implications of Gene Gain and Loss in the Evolution of Prochlorococcus. PLoS Genetics 3: e231.

Maldonado MT, Price NM (2001) Reduction and transport of organically bound iron by Thalassiosira Oceanica (Bacillariophyceae) Journal of Phycology 37: 298-310.

Mann EL, Ahlgren N, Moffett JW, Chisholm SW (2002) Copper Toxicity and Cyanobacteria Ecology in the Sargasso Sea. Limnology and Oceanography 47: 976-988.

Mann EL, Chisholm SW (2000) Iron limits the cell division rate of Prochlorococcus in the eastern equatorial Pacific. Limnology and Oceanography 45: 1067-1076.

Martiny AC, Coleman ML, Chisholm SW (2006) Phosphate acquisition genes in Prochlorococcus ecotypes: Evidence for genome-wide adaptation. Proceedings of the National Academy of Sciences 103: 12552-12557.

Moore JK, Doney SC, Lindsay K (2004) Upper ocean ecosystem dynamics and iron cycling in a global three-dimensional model. Global Biogeochemical Cycles 18: GB4028.

Moore LM, Coe AC, Zinser ER, Saito MA, Sullivan MB, Lindell D, Frois-Moniz K, Waterbury W, Chisholm SW (2007) Culturing the marine cyanobacterium Prochlorococcus. Limnology and Oceanography Methods 5: 353-362. 
Moore LR, Chisholm SW (1999) Photophysiology of the Marine Cyanobacterium Prochlorococcus: Ecotypic Differences among Cultured Isolates. Limnology and Oceanography 44: 628-638.

Morel FMM, Hudson RJM, Price NM (1991) Limitation of Productivity by Trace Metals in the Sea. Limnology and Oceanography 36: 1742-1755.

Nishioka J, Takeda S, de Baar HJW, Croot PL, Boye M, Laan P, Timmermans KR (2005) Changes in the concentration of iron in different size fractions during an iron enrichment experiment in the open Southern Ocean. Marine Chemistry 95: 51-63.

Nishioka J, Takeda S, Wong CS, Johnson WK (2001) Size-fractionated iron concentrations in the northeast Pacific Ocean: distribution of soluble and small colloidal iron. Marine Chemistry 74: 157-179.

Oschlies A, Garcon V (1998) Eddy-induced enhancement of primary production in a model of the North Atlantic Ocean. Nature 394: 266-269.

Palenik B, Ren Q, Dupont CL, Myers GS, Heidelberg JF, Badger JH, Madupu R, Nelson WC, Brinkac LM, Dodson RJ, Durkin AS, Daugherty SC, Sullivan SA, Khouri H, Mohamoud Y, Halpin R, Paulsen IT (2006) Genome sequence of Synechococcus CC9311: Insights into adaptation to a coastal environment. Proceedings of the National Academy of Sciences 103: 13555-13559.

Price NM, Harrison GI, Hering JG, Hudson RJ, Nirel PMV, Palenik B, Morel FMM (1988) Preparation and chemistry of the artifical algal culture medium Aquil. Biological Oceanography 6: 443-461

Raven JA (1988) The Iron and Molybdenum use Efficiencies of Plant Growth with Different Energy, Carbon and Nitrogen Sources. New Phytologist 109: 279-287.

Rocap G, Larimer FW, Lamerdin J, Malfatti S, Chain P, Ahlgren NA, Arellano A, Coleman M, Hauser L, Hess WR, Johnson ZI, Land M, Lindell D, Post AF, Regala W, Shah M, Shaw SL, Steglich C, Sullivan MB, Ting CS, Tolonen A, Webb EA, Zinser ER, Chisholm SW (2003) Genome divergence in two Prochlorococcus ecotypes reflects oceanic niche differentiation. Nature 424: 1042-1047.

Rue EL, Bruland KW (1995) Complexation of iron(III) by natural organic ligands in the Central North Pacific as determined by a new competitive ligand equilibration/adsorptive cathodic stripping voltammetric method. Marine Chemistry 50: 117-138.

Rue EL, Bruland KW (1997) The Role of Organic Complexation on Ambient Iron Chemistry in the Equatorial Pacific Ocean and the Response of a Mesoscale Iron Addition Experiment. Limnology and Oceanography 42: 901-910.

Saito MA, Moffett JW, Chisholm SW, Waterbury JB (2002) Cobalt limitation and uptake in Prochlorococcus. Limnology and Oceanography 47: 1629-1636.

Saito MA, Schneider DL (2006) Examination of precipitation chemistry and improvements in precision using the $\mathrm{Mg}(\mathrm{OH}) 2$ preconcentration inductively coupled plasma mass spectrometry (ICP-MS) method for high-throughput analysis of open-ocean Fe and Mn in seawater. Analytica Chimica Acta 565: 222-233.

Shaked Y, Kustka AB, Morel FMM (2005) A general kinetic model for iron acquisition by eukaryotic phytoplankton. Limnology and Oceanography 50: 872-882.

Sherrell RM, Boyle EA (1988) Zinc, chromium, vanadium and iron in the Mediterranean Sea. Deep Sea Research Part A Oceanographic Research Papers 35: 1319-1334.

Singh AK, Sherman LA (2000) Identification of Iron-Responsive, Differential Gene Expression in the Cyanobacterium Synechocystis sp. Strain PCC 6803 with a Customized Amplification Library. Journal of Bacteriology 182: 3536-3543.

Sunda W, Huntsman SA (1995) Iron uptake and growth limitation in oceanic and coastal phytoplankton. Marine Chemistry 50: 189-206.

Sunda W, Huntsman SA (1997) Interrelated influence of iron, light and cell size on marine phytoplankton growth. Nature 390: 389-392.

van den Berg CMG (1995) Evidence for organic complexation of iron in seawater. Marine Chemistry 50: 139-157.

Webb EA, Moffett JW, Waterbury JB (2001) Iron Stress in Open-Ocean Cyanobacteria (Synechococcus, Trichodesmium, and Crocosphaera spp.): Identification of the IdiA Protein. Appl Environ Microbiol 67: 5444-5452. 
Weber L, Volker C, Oschlies A, Burchard H (2007) Iron profiles and speciation of the upper water column at the Bermuda Atlantic Time-series Study site: a model based sensitivity study. Biogeosciences 4: 689-706.

Welsh EA, Liberton M, Stöckel J, Loh T, Elvitigala T, Wang C, Wollam A, Fulton RS, Clifton SW, Jacobs JM, Aurora R, Ghosh BK, Sherman LA, Smith RD, Wilson RK, Pakrasi HB (2008) The genome of Cyanothece 51142, a unicellular diazotrophic cyanobacterium important in the marine nitrogen cycle. Proceedings of the National Academy of Sciences 105: 15094-15099.

Wu J, Boyle E, Sunda W, Wen L-S (2001) Soluble and Colloidal Iron in the Oligotrophic North Atlantic and North Pacific. Science 293: 847-849.

Wu J, Luther GW (1995) Complexation of Fe(III) by natural organic ligands in the Northwest Atlantic Ocean by a competitive ligand equilibration method and a kinetic approach. Marine Chemistry 50: 159-177. 

CHAPTER THREE

Prochlorococcus whole-genome response to changes in iron availability

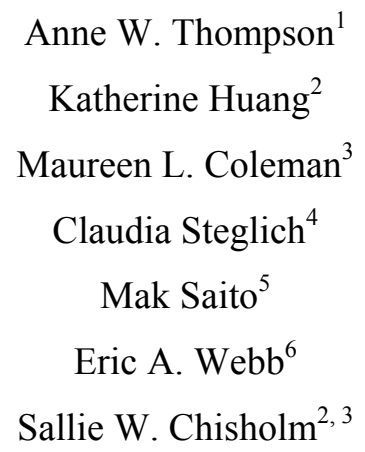




\begin{abstract}
Prochlorococcus contributes significantly to primary productivity in the oligotrophic open ocean and though it is abundant in regimes of exceeding low iron concentrations, iron availability can limit its growth rate. The minimum iron concentration required for growth differs between the closely related Prochlorococcus strains MED4 and MIT9313 suggesting that iron metabolism is a source of diversity among all Prochlorococcus. We examined the effects of changing iron availability on whole-genome expression in MED4 and MIT9313 in order to unveil aspects of iron acquisition, use, and regulation in Prochlorococcus. Both strains responded strongly to iron starvation and recovery from iron starvation at the transcriptional level within 12 hours while growth rates remained maximal. About 100 genes were differentially expressed in each MED4 and MIT9313. Though the genomes of MED4 and MIT9313 share more than 1200 genes, only three of these orthologs were clearly differentially expressed in both strains in response to changes in iron availability. Thus, we find that the iron-stress response is encoded primarily in the non-core genome of Prochlorococcus. Also, we observe that the expression response to light stress is similar to the iron-starvation response, emphasizing the importance of iron in light-harvesting and photosynthesis in Prochlorococcus.
\end{abstract}

\title{
Introduction
}

Prochlorococcus is a unicellular cyanobacterium that contributes significantly to primary productivity and constitutes a majority of the phytoplankton community in the tropical and subtropical open oceans (Partensky et al, 1999). Though the link between primary productivity and iron availability has been increasingly recognized, the role of Prochlorococcus in iron biogeochemical cycling in the ocean remains to be described (Boyd et al, 2007; Moore et al, 2004; Tortell, 1999). Prochlorococcus is known to be iron-limited in some oceanic regimes, such as the Equatorial Pacific, despite its small size which often confers an advantage in nutrient acquisition over larger phytoplankton (Mann and Chisholm, 2000; Morel and Price, 2003). Iron limitation of Prochlorococcus in the natural environment suggests that iron availability is important in determining the productivity and abundance of Prochlorococcus on a global scale along with factors such as light, temperature, phage susceptibility, grazing, and macro-nutrient availability (Johnson et al, 2006; Martiny et al, 2006; Moore, 2004). It will be critical to describe the relationship between Prochlorococcus and iron if we are to better understand biogeochemical cycling of iron in the open ocean and the ecology of Prochlorococcus.

Like other cyanobacteria, we expect the metabolism of Prochlorococcus to rely heavily on iron because its ancestors likely evolved in a reducing environment where iron availability was high (Anbar and Knoll, 2002; Hunter and Boyd, 2007; Saito et al, 2003). Coupled with the relative ease of reduction and oxidation of the ferrous and ferric forms of iron under intracellular conditions, iron was an ideal element to catalyze electron transfer reactions in proteins in the 
early evolution of life and with only a few exceptions, it is required in all forms of life (Posey and Gherardini, 2000).

In contrast to the iron-rich conditions in which the ancestors of cyanobacteria originated, the concentrations of dissolved iron in the modern open ocean are extremely low, in the nanomolar range, due to oxidizing conditions that lead to the formation and precipitation of Fe(III) hydroxides (Johnson et al, 1997; Martin et al, 1990a; Martin et al, 1993; Martin and Gordon, 1988; Martin et al, 1990b; Martin et al, 1989; Morel et al, 2003). Also, iron concentrations vary greatly - temporally and spatially - over the horizontal and vertical dimensions of Prochlorococcus' habitat, the euphotic zone. Furthermore, there is now consensus that organic ligands bind most of the dissolved iron in the open ocean (Hunter et al, 2007; Rue and Bruland, 1997). Yet, the biological availability of iron complexed to organic ligands remains an open question. Despite these dramatic changes in the speciation of iron in the ocean over time, and the seeming difficulty of iron acquisition from the perspective of a free-living cell in the dilute environment of the open ocean, iron remains an essential nutrient in the metabolism of Prochlorococcus and other marine cyanobacteria.

\section{The role of iron in cellular metabolism}

The most well characterized role of iron within cyanobacteria is as a cofactor in photosynthetic electron transport where the photosystem I (PSI) complex, photosystem II (PSII) complex, ferredoxin, and the cytochromes all contain iron (Morel, 1991; Raven, 1988). In other organisms iron is also known to be required for redox reactions in chlorophyll synthesis, reduction and assimilation of nitrogen species, and in protection from oxidative stress (BermanFrank et al, 2001; Michel, 2004; Raven, 1988).

Recently, it has been recognized that iron plays non-electron transfer roles within the cell especially in the context of iron-sulfur clusters (Beinert et al, 1997). For example, the [4Fe-4S] cluster of Escherichia coli endonuclease III appears to play a role in the DNA binding properties of the enzyme rather than its catalytic activity (Thayer, 1995). An [Fe-S] cluster has also been implicated in the sequestration and transport of the molybdenum required in the active site of nitrogenase for nitrogen fixation (Dos Santos and Dean, 2008; Hernandez et al, 2008). Other roles for Fe-S clusters include acting as environmental sensors for oxygen availability and in the generation of radicals (Dos Santos et al, 2008). 


\section{The iron stress response}

The iron-stress responses of several different freshwater and oceanic cyanobacteria, including Prochlorococcus, have been studied in an effort to better understand the cellular role for iron in cyanobacteria and the method by which they acquire iron and hence influence iron biogeochemical cycles. The physiological manifestations of iron deprivation in cyanobacteria include loss of pigmentation (chlorosis), changes in the fluorescence/absorbance wavelengths of chlorophyll a, reduction in cell size, and generally-reduced photosynthetic efficiency (Ferreira and Straus, 1994). Oxidative stress is also a common feature of the iron stress response in cyanobacteria and other bacteria and is caused by the interaction of free iron with superoxide and hydrogen peroxide within the cell to produce hydroxyl radicals that can cause severe damage (Andrews et al, 2003; Touati, 2000).

At the molecular level, cyanobacteria and other bacteria are known to adjust to low iron availability in two ways (Ferreira et al, 1994). The first mechanism of dealing with low iron availability is through a concerted reduction of the cellular iron requirement, or iron quota. This intracellular adjustment is exemplified by the replacement of the iron-containing electron transfer protein ferredoxin with the iron-free protein flavodoxin in prokaryotic and eukaryotic photosynthetic microorganisms (Meimberg et al, 1999; Sancho, 2006; Zurbriggen et al, 2007). Another example of how a photosynthetic cell can reduce its iron quota is by remodeling of the iron-rich PSI complex during iron stress (Bibby et al, 2003; Moseley et al, 2002). Iron could then be sequestered by the iron-storage molecule ferritin and subsequently released for other cellular processes (Busch et al, 2008).

The second basic response of cyanobacteria and other bacteria to iron stress is through enhancing iron uptake from the environment. Some cyanobacteria, such as Anabaena sp. are capable of producing siderophores during iron stress to enhance the solubility of iron in the surrounding environment and facilitate uptake (Ferreira et al, 1994; Goldman et al, 1983; Wilhelm, 1995). Prochlorococcus itself may be capable of producing organic ligands to bind cobalt, cobalophores, in conditions of low cobalt availability (Saito et al, 2002). In E.coli the transport of iron bound to organic ligands depends greatly on the iron-stress conditions and expression of siderophore-specific receptors on the outer membrane (Buchanan et al, 1999; Ferguson et al, 2002; Ferguson et al, 1998). Similarly, some cyanobacteria appear capable of transporting organically-bound iron with a dependence on the specific type of organic complex that is available (Hutchins et al, 1999). Expression of homologs to high-affinity siderophore 
uptake systems is also apparent in several types of cyanobacteria, yet so far, no recognizable siderophore synthesis or transport genes, or a complete iron-uptake system, have been recognized in Prochlorococcus (Rocap et al, 2003; Webb et al, 2001). Reduction of iron from organic ligands at the cell surface is another mechanism by which a cell in an oxic environment can enhance iron uptake in response to low availability and this capability has been shown in diatoms (Shaked et al, 2005) but is not yet discovered in prokaryotes.

\section{Regulation of iron metabolism}

The predicted high iron-requirement of cyanobacteria (Raven, 1988), coupled with the low availability of iron in the open ocean and the threat of oxidative damage posed by free iron within the cell, suggests a high-affinity and tightly-regulated iron acquisition and storage system in Prochlorococcus and other open-ocean cyanobacteria. Coordination and regulation of cellular iron metabolism has been attributed to the ferric uptake regulator (fur), first characterized in E.coli (Ernst et al, 1978; Hantke, 1981). Fur homologs also exist in Prochlorococcus and other marine cyanobacteria and are expressed in Prochlorococcus (Rocap et al, 2003; Zinser et al, 2009). Recently, a role for small RNAs (sRNA) in regulating cellular iron metabolism was described in E.coli and Pseudomonas aeruginosa, which involves the regulation of the sRNA ryhB by fur to control the expression of genes that encode iron-using proteins (Masse and Gottesman, 2002; Masse et al, 2005; Wilderman et al, 2004). In the cyanobacterium Synechocystis 6803, the iron stress-repressed RNA (IsrR) has been found to regulate the expression of the iron stress-induced protein A (isiA) (Duhring et al, 2006). Furthermore, several sRNAs have been recently identified in Prochlorococcus MED4 and respond significantly to shifts in light availability (Steglich et al, 2008). Given the intimate connection between light and iron metabolism in cyanobacteria, a role for small RNAs in regulating iron metabolism in Prochlorococcus is also likely.

\section{Prochlorococcus whole-genome response to iron availability}

Here, we report on the whole-genome transcription response of Prochlorococcus strains MED4 and MIT9313 to short-term iron deprivation and rescue from iron deprivation.

Prochlorococcus MED4 and MIT9313 represent the diversity of the cultured representatives of Prochlorococcus in terms of phylogeny, light and nutrient physiology, origin of isolation, and pigment composition so may represent a range of possible responses to iron deprivation in 
Prochlorococcus. Given that MIT9313 is adapted for living in low light where light-harvesting efficiency is important, we would expect MIT9313 to have a higher iron requirement, i.e. higher $\mathrm{Fe}: \mathrm{C}$ ratio. Additionally, given that MIT9313 has a higher surface area to volume ratio than MED4 we anticipate that acquiring enough iron to meet its cellular requirements is more difficult in MIT9313. Curiously, however, MIT9313 is capable of survival at lower iron concentrations than MED4 in culture (Chapter 2, Figure 4). Thus, we regard the analysis of the transcriptional response of MED4 and MIT9313 to iron stress in this study as an opportunity to explain the differences in iron physiology of these two strains.

Our transcriptional analysis will focus on the how each strain responds to iron stress individually and then compare the response of both strains to each other. First we ask: Which genes in Prochlorococcus respond to sudden iron deprivation and resuscitation? To this end we will focus on genes predicted to be involved in iron transport, use, or regulation in each strain. Then we compared MED4 and MIT9313 to each other and to the other sequenced isolates examined in Kettler et al. 2007 both in terms of genome content and position of iron-responsive genes along the genome and asked: Is iron metabolism encoded in the core genome or noncore genome of Prochlorococcus? Further, we contrasted the iron-stress expression response to the other stress responses of nitrogen starvation (Tolonen et al, 2006), phosphorus starvation (Martiny et al, 2006), diel light availability (Zinser et al, 2009), shifts in light quality and quantity (Steglich et al, 2006), and phage infection (Lindell et al, 2007) and asked: Which genes are involved in an iron-specific response rather than a general stress response? By addressing these questions we aim to understand how iron plays a role in defining the Prochlorococcus group and in contributing to the diversity of its ecotypes.

\section{Methods}

\section{Iron-deprivation culture conditions}

Prochlorococcus strains MED4 and MIT9313 were grown in PRO99 media modified for trace metal clean work (TM-PRO99) (Saito et al, 2002). Modifications included microwave sterilization of trace metal clean oligotrophic seawater (collected with a Teflon- coated diaphragm pump in a positive pressure trace metal clean bubble), an increase in the concentration of EDTA to $11.7 \mu \mathrm{m}$, and treating major nutrients $\left(\mathrm{NHCl}_{4}\right.$ and $\left.\mathrm{NaH}_{2} \mathrm{PO}_{4}\right)$ with chelex prior to sterile filtration and addition to media (Keller et al, 1988; Moore et al, 2007; Price et al, 1988; Saito et $a l, 2002)$. In addition, iron-replete concentrations were reduced from $1 \mu \mathrm{M} \mathrm{FeCl}_{3}$ in $\mathrm{PRO} 99$ 
(Moore et al, 2007) to 1nM and 100nM, for MED4 and MIT9313 respectively, to help reduce carryover in the transfer to iron-free media and to accelerate iron starvation. All media preparations were conducted in a level 100 trace metal clean room (Woods Hole Oceanographic Institution, Woods Hole, MA). Cultures were grown in polycarbonate bottles (Nalgene) that were soaked in $0.1 \%$ citranox (brand) for 24 hours, 10\% HCL (Baker Instra-nalyzed) for 24 hours, and then rinsed in $\mathrm{pH} 2$ Milli-Q water. MED4 was grown at continuous light at an irradiance of $27 \mu \mathrm{Em}^{-2} \mathrm{~s}^{-1}$ and at $21^{\circ} \mathrm{C}$. Prochlorococcus MIT9313 was grown at continuous light at an irradiance of $20 \mu \mathrm{E} \mathrm{m}^{-2} \mathrm{~s}^{-1}$ and at $25^{\circ} \mathrm{C}$.

To induce abrupt iron starvation, triplicate $4 \mathrm{~L}$ volume cultures were harvested by centrifugation (8500 rpm), washed twice with iron-free media, then split in two parts and resuspended in either iron replete TM-PRO99 (see above) or iron-free TM-PRO99. After two days in iron-free media, MIT9313 cultures were transferred a second time into iron-free media to reduce carry-over and iron storage before centrifugation, rinsing, and re-suspension and the start of sampling. Cultures were sampled for RNA and flow cytometry at 0, 12, 24, 48, and 70 hours (MED4) and at 0, 16, 28, 53, and 72 hours (MIT9313). From this point on, the time-points in the MIT9313 experiment will be called by the hours in the MED4 experiment $(0,12,24,48$, and 70), for simplicity. Iron was added back to the iron-deprived cultures to match the iron-replete concentration for MED4 at 49 hours and MIT9313 at 54 hours after initial iron deprivation. For both strains, additional samples were collected for flow cytometry, fluorescence, and quantitative RT-PCR at selected time-points.

Growth rates were calculated from changes in bulk fluorescence measured by fluorometry (Picofluor by Turner Biosystems) and cell number measured by an Influx cell sorter (Cytopeia). The cellular parameters of cell size and chlorophyll per cell were approximated by normalizing forward angle light scatter (FALS) and red fluorescence per cell to $2 \mu \mathrm{m}$-diameter standard Fluoresbrite beads (Polysciences, Inc) when interrogated by a blue laser using an Influx cell sorter (Cytopeia).

\section{Photochemical conversion efficiency $\left(F_{\sqrt{ }} / F_{m}\right)$}

Photochemical conversion efficiency (or, $\mathrm{F}_{\mathrm{v}} / \mathrm{F}_{\mathrm{m}}$ ) was measured for MED4 to serve as an indicator of iron starvation (Falkowski and Raven, 2007). $\mathrm{F}_{\mathrm{v}} / \mathrm{F}_{\mathrm{m}}$ was measured using 3-(3.4Dichlorophenyl)-1.1-dimethylurea (DCMU, or Diuron) (Sigma) additions to culture samples. $5 \mu 1$ of $1 \mathrm{mM}$ DCMU dissolved in ethanol or $5 \mu 1$ ethanol (control) was added to duplicate $300 \mu 1$ 
samples of culture in a black-sided, clear-bottom 96-well plate, and mixed well by pipetting. The plate was incubated for 10 minutes in the ambient light of the laboratory and then fluorescence was measured in a 96-well plate reader (Biotek). $\mathrm{F}_{\mathrm{v}} / \mathrm{F}_{\mathrm{m}}$ was calculated as:

$$
\mathrm{F}_{\mathrm{v}} / \mathrm{F}_{\mathrm{m}}=\text { (fluorescence with DCMU - fluorescence of ethanol control) }
$$

(fluorescence with DCMU)

\section{RNA preparation}

Depending on the time-point and estimated cell density, 200ml-400ml cultures were sampled for RNA by centrifugation $(8500 \mathrm{rpm})$ for $8-10$ minutes, then were re-suspended in $1 \mathrm{ml}$ of RNA storage buffer (200mM sucrose, $10 \mathrm{mM} \mathrm{NaOAc} \mathrm{pH} \mathrm{5.2,} \mathrm{5mM} \mathrm{EDTA} \mathrm{pH5.2),} \mathrm{flash}$ frozen in liquid nitrogen, and stored at $-80^{\circ} \mathrm{C}$. Prior to RNA extraction, MIT9313 cells were treated with $9 \mu \mathrm{g} / \mu 1$ lysozyme for 1 hour at $37^{\circ} \mathrm{C}$ to enhance cellular lysis and improve RNA yields (Tolonen et al, 2006). SuperaseIN (Ambion) was added to the lysis reactions with each addition of lysozyme to protect RNA from degradation. The mirVana miRNA kit (Ambion) was used to extract RNA following the manufacture's protocol. DNA was removed from the eluted RNA using Turbo DNase (Ambion). RNA concentrations were measured using a Nanodrop (Thermo Scientific) and by using the nucleic acid dye, Ribogreen (Molecular Probes). On average, $\sim 20 \%$ of nucleic acids were lost following DNAse treatment.

\section{RNA amplification}

Due to poor RNA yield, DNase treated MIT9313 RNA samples were concentrated using RNA-secure (Ambion) treated - Micron Y-30 columns (Millipore) then amplified using the Message Amp TM II-Bacteria Prokaryotic RNA Amplification Kit (Ambion) according to the manufacturer's protocol with a 10hour linear amplification step. rRNA genes have been found to amplify non-linearly and so were excluded from the analysis for MIT9313 (Frias-Lopez et al, 2008). Otherwise, in a test comparing differential gene expression in response to phosphorus limitation of MIT9313, fold change between experimental and control treatments for both amplified RNA and non-amplified RNA were consistent (Frias-Lopez et al, 2008).

\section{Quantitative reverse transcription PCR}

Unamplified MED4 RNA (10ng) was reverse transcribed using 200 units of SuperScript II (Invitrogen) with 10 units of SuperaseIN (Ambion). Primer sequences for the genes isiB and $r n p B$ are presented in Table 1. Real-time PCR reactions were performed in duplicate with the 
resulting cDNA diluted 5-fold in 10mM Tris, $\mathrm{pH} 8$, using the Qiagen SYBR green kit. An MJ Research Opticon DNA engine was used for the real-time PCRs with the following program: 15 minutes at $95^{\circ} \mathrm{C}$, then 40 cycles of denaturation $\left(95^{\circ} \mathrm{C}, 15\right.$ seconds), annealing $\left(56^{\circ} \mathrm{C}, 30\right.$ seconds), and extension $\left(72^{\circ} \mathrm{C}, 30\right.$ seconds), followed by 5 minutes at $72^{\circ} \mathrm{C}$. cDNA for isiB was quantified relative to $r n p B$ using the $\Delta-\Delta \mathrm{C}_{\mathrm{T}}$ method (Livak and Schmittgen, 2001).

\begin{tabular}{|l|l|l|}
\hline \multicolumn{3}{|l|}{ Table 1. Primers used for quantitative reverse-transcriptase PCR analysis of $\boldsymbol{r n p B}$ and $\boldsymbol{i s i B}}$. \\
\hline Target gene & Primer name & Oligonucleotide sequence 5'-3' \\
\hline rnpB - MED4 and MIT9313 & rnpBFWMIT9313+MED4 & TTG AGG AAA GTC CGG GCT C \\
\hline rnpB - MED4 & rnpBRVMED4 & GCG GTA TGT TTC TGT GGC ACT \\
\hline isiB - MED4 & isiBMedFW & CTA CAT GGA ATA CTG GAG CAG \\
\hline isiB - MED4 & isiBMedRV & CAT AGC CAA CCA TTT CAG CAC \\
\hline
\end{tabular}

\section{Array Normalization and Analysis}

The custom-made Affymetrix array, MD-9313, was used for measuring whole-genome expression. Technical details for the MD-9313 array can be found in Lindell et al 2007. Duplicate (MED4) or triplicate (MIT9313) samples were hybridized to the arrays and processing (cDNA synthesis, labeling, hybridization, staining, and scanning) was carried out by the Harvard Biopolymers Facility (Cambridge, MA) following the Affymetrix protocols for E.coli with $2 \mu \mathrm{g}$ RNA. In choosing a normalization approach, we assume that most of the genes in the genome do not change with iron deprivation.

The normalization method choosen here is based on recommendations published by Choe et al. 2005. Background correction was accomplished using RMA (robust multi-array analysis) implemented in Matlab (Mathworks) and using a library file that was updated in September 2008 to include recently discovered ORFs and non-coding RNAs (MD4-9313a520062.Sep08.cdf). The use of the updated library file contrasts with the library files used in Prochlorococcus array data published to date (Lindell et al, 2007; Martiny et al, 2006; Steglich et al, 2006; Tolonen et al, 2006). Loess normalization at the probe-set level was implemented in Matlab (Mathworks) to correct for the influence of signal intensity on fold change for each probe-set, including those that represent intergenic regions (Choe et al, 2005).

Six comparisons were made for each strain to assess the response of Prochlorococcus MED4 and MIT9313 to iron availability. Experimental replicates (-Fe treatment) were compared to control replicates ( $+\mathrm{Fe}$ treatment) at each of the five time-points taken at $0,12,24,48$, and 70 
hours. Note that the 70 hour time-point still compares experimental treatment to control but iron has been added to the experimental treatment to match control concentrations (see above). We made a sixth comparison between the experimental treatment before iron addition (48 hours -48 . $\mathrm{Fe}$ ) and the experimental treatment after iron addition $\left(70\right.$ hours $\left.-70{ }^{*}{ }_{\mathrm{Fe}}\right)$ which we refer to as the experimental treatment response to iron rescue.

Bayesian statistical analysis for each comparison was performed in Cyber-T (http://cybert.microarray.ics.uci.edu/index.shtml) to identify differentially expressed genes (Baldi and Long, 2001). Window sizes of 81 and 101 were used for MED4 and MIT9313, respectively, to calculate the Bayesian estimate of variance. Confidence intervals were set at 3-times the number of replicates for each strain, timepoint, and treatment condition. The program Q-VALUE (http://genomics.princeton.edu/storeylab/qvalue/), implemented in Matlab (Mathworks), was used to calculate the false discovery rate (q-value) for each probe-set (Storey and Tibshirani, 2003).

\section{Identification of differentially expressed genes}

Differentially expressed genes were identified based on their q-value (i.e. false discovery rate) and magnitude of fold-change relative to the control. We plotted q-value against absolute value of fold change for each comparison in the MED4 and MIT9313 experiments in order to determine the most appropriate thresholds to use in the identification of differentially expressed genes (Appendix B). Differentially expressed genes (DEG) were defined as those with q-values less than 0.01 and $\log _{2}$ (fold change) greater than 1 or less than -1 . Previous work demonstrates that Affymetrix microarrays underestimate fold change (Choe et al, 2005), thus we kept track of genes with $\log _{2}$ (fold change) greater than 0.8 or less than -0.8 in any comparison and refer to them as marginally differentially expressed genes if there is anything noteworthy about them.

\section{Cluster analysis}

Hierarchical clustering was performed with $\log _{2}$ transformed fold change for every nonintergenic probe-set (hereafter, referred to as genes) in every comparison from the MED4 and MIT9313 experiments. The program Cluster (Eisen et al, 1998) was used to perform hierarchical clustering of genes and arrays using Complete Linkage Clustering and Correlation (centered) chosen as the similarity metric. The program TreeView (http://rana.lbl.gov/EisenSoftware.htm) was used to visualize and analyze cluster results. Following cluster analysis, only differentially expressed genes were considered in the description and interpretation of each cluster. 


\section{Comparison to other experiments using the custom M4-9313 Affymetrix array}

The global gene expression response of Prochlorococcus MED4 and MIT9313 has been published elsewhere for growth during shifts in light availability and wavelength, nitrogen starvation, phosphorus starvation, and phage infection (Kettler et al, 2007; Lindell et al, 2007; Martiny et al, 2006; Steglich et al, 2006; Tolonen et al, 2006; Zinser et al, 2006; Zinser et al, 2009). Since the publishing of these results, our group has updated the Affymetrix library file for the custom M4-9313 microarray to include newly discovered orfs and small non-coding RNAs published in Steglich et al. 2006 and Kettler et al. 2007. In order to thoroughly compare the results of this study, which applies the updated library file, to the other experiments, it was necessary to re-analyze the previously published microarray data using the updated library file. Normalization and analysis of the previously published datasets was carried out with the same tools and methods as the current study including RMA and loess normalizations as described above under Array Normalization and Analysis. We examined q-value vs. fold change to determine which thresholds for significance are appropriate for determining differential gene expression in each experiment (Appendix B, Figures 1-8). With the exception of the nitrogen experiment, where we increased the fold change threshold because several hundreds of genes were above the original, we implemented the same thresholds for significance that were used in the original published works (Appendix B, Figures 1-8).

\section{Results and Discussion \\ Iron deprivation physiology}

Division rates were the same for the iron-replete control $(+\mathrm{Fe})$ and iron-free experimental treatment (-Fe) during iron deprivation of MIT9313 and up until 48 hours post-iron deprivation for MED4 after which the growth rate of the MED4 experimental cultures decreased slightly (Figure 1). The steady growth rate of both strains during iron stress contrasts with studies on nitrogen and phosphorus starvation in Prochlorococcus where growth rates decreased dramatically during starvation (Martiny et al, 2006; Tolonen et al, 2006). Relative red fluorescence per cell and relative forward-angle light scatter (proxy for cell size) decreased in iron-deprived cultures compared to the iron-replete control cultures indicating iron stress in the experimental treatment (Figure 2). Both MED4 and MIT9313 displayed these physiological signs of iron stress within 20 hours of initial iron deprivation (Figure 1 and 2). The decrease in red 

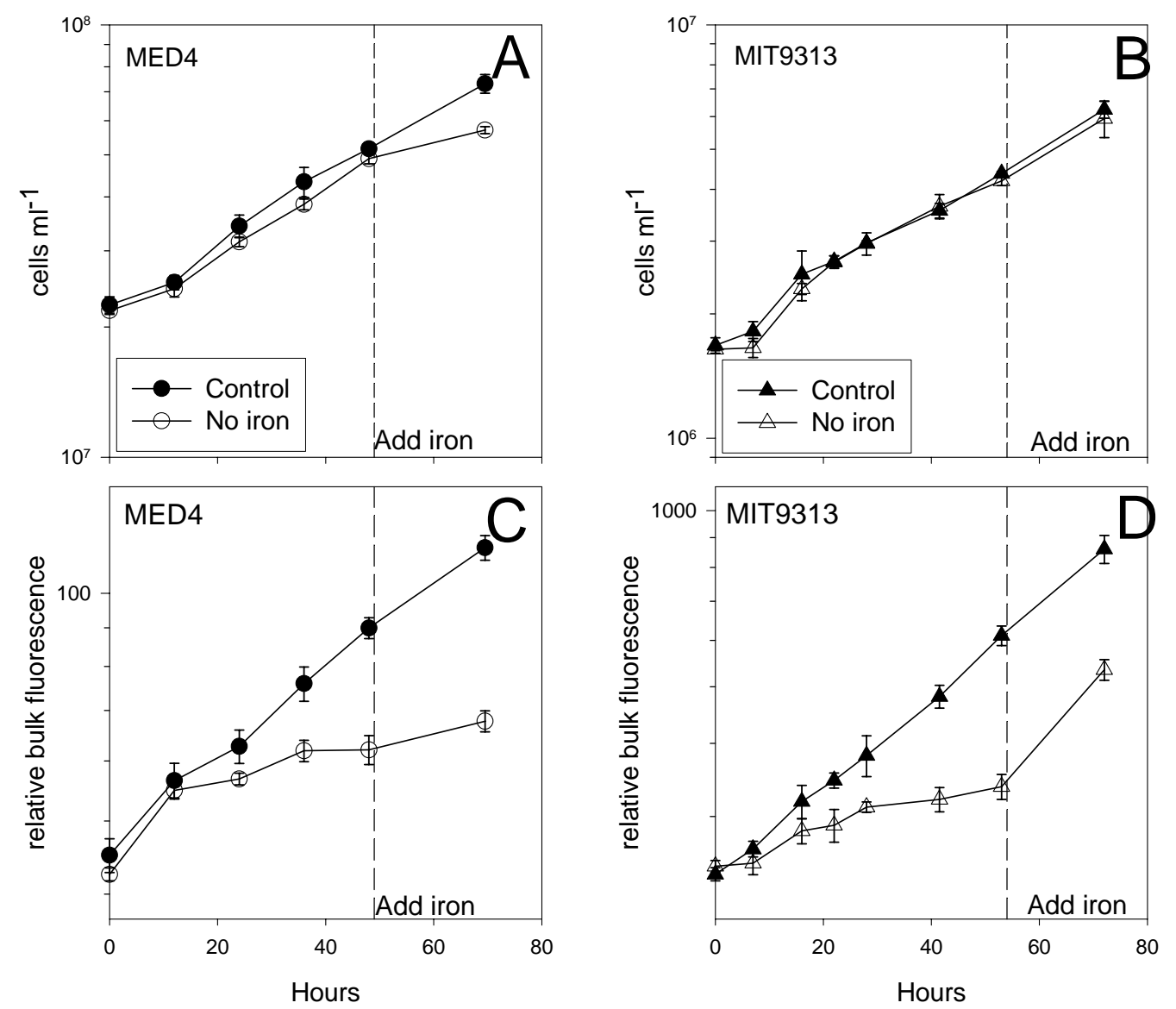

Figure 1: Growth of Prochlorococcus MED4 and MIT9313 over a time course of iron deprivation and recovery from iron starvation. The hours $=0$ time-point was measured after cells were centrifuged and resuspended in either + Fe or - Fe media. (A, B) MED4 and MIT9313 changes in cell concentration over time in control and experimental (no iron) treatments. (C, D) MED4 and MIT9313 relative bulk fluorescence over time in experimental (no iron) and control treatments. Dotted line indicates iron addition to the experimental (no iron) treatment cultures to match iron concentrations in the control treatment. 

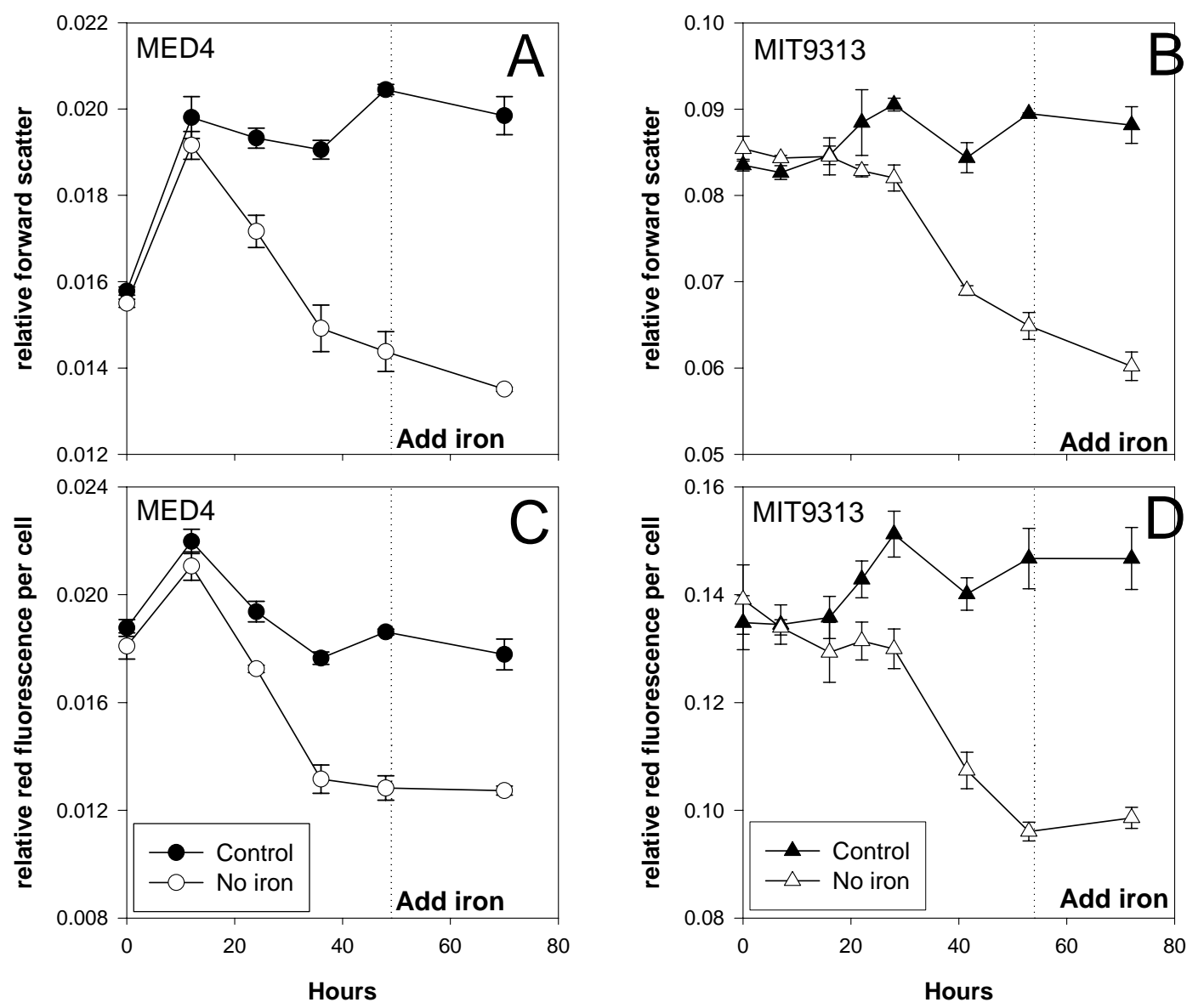

Figure 2: Changes in mean relative red fluorescence per cell and mean relative forward scatter per cell in individual MED4 and MIT9313 cells over the time course of iron deprivation and recovery from iron starvation. (A,B) MED4 and MIT9313 forward scatter relative to standard beads as a proxy for cell size. (C,D) MED4 and MIT9313 red fluorescence per cell relative to standard beads over the time course of iron deprivation. Dotted line indicates iron addition to the experimental (no iron) treatment to match the iron concentration of the control treatment. 
fluorescence per cell is consistent with chlorosis seen in cyanobacteria and in other photosynthetic organisms during iron stress. The decrease in cell size is consistent with the idea that a reduced surface area to volume ratio facilitates more efficient iron uptake and agrees with other published data on Prochlorococcus in the field as well as marine diatoms and dinoflagellates (Cavender-Bares et al, 1999; Mann et al, 2000; Sunda and Huntsman, 1995). Because cell division rates between the control and experimental treatments did not diverge while cell size decreased, it appears that cell division was favored over cellular growth, which would serve to increase the cell's surface area to volume ratio.

After addition of iron to the experimental treatments "iron rescue" (at 49 and 54 hours for MED4 and MIT9313, respectively) bulk fluorescence increased in both MED4 and MIT9313 within 24 hours. Red fluorescence per cell did not increase with iron addition in MED4, though in MIT9313 it rebounded slightly (Figure 2). Interestingly, cell size continued to decrease in both MED4 and MIT9313 cultures after iron rescue, which suggests that cells respond immediately to increased iron availability by remodeling their light harvesting systems rather than increasing in cell size. We hypothesize that given more time we would observe a cell size increase in both MED4 and MIT9313 with the iron addition.

Photochemical conversion efficiency $\left(\mathrm{F}_{\mathrm{v}} / \mathrm{F}_{\mathrm{m}}\right)$ was used to measure the response to iron starvation in MED4 (Figure 3a). Between 12 and 24 hours after the initiation of iron starvation, the $\mathrm{F}_{\mathrm{v}} / \mathrm{F}_{\mathrm{m}}$ of the control and no-iron treatment conditions diverged significantly indicating a lower photochemical efficiency in the iron-deprived culture. The diminished photosynthetic efficiency in the iron-stressed cultures is consistent with the decrease in red fluorescence per cell, cell size, and bulk fluorescence indicated at the onset of iron stress in MED4.

To confirm that the physiological changes discussed above indicate iron stress in Prochlorococcus and to determine the timing of the iron-stress response, quantitative reverse transcriptase PCR (qRT-PCR) was used to measure the expression of the iron-stress induced gene, flavodoxin (isiB), relative to the expression of the house-keeping gene rnpB in MED4. Within 12 hours of exposure to iron-free media, isiB expression was significantly up-regulated relative to $r n p B$ in the experimental (-Fe) condition compared to the control. Our detection of isiB expression is consistent with other work showing its up-regulation during iron stress in Prochlorococcus (Bibby et al, 2003). 

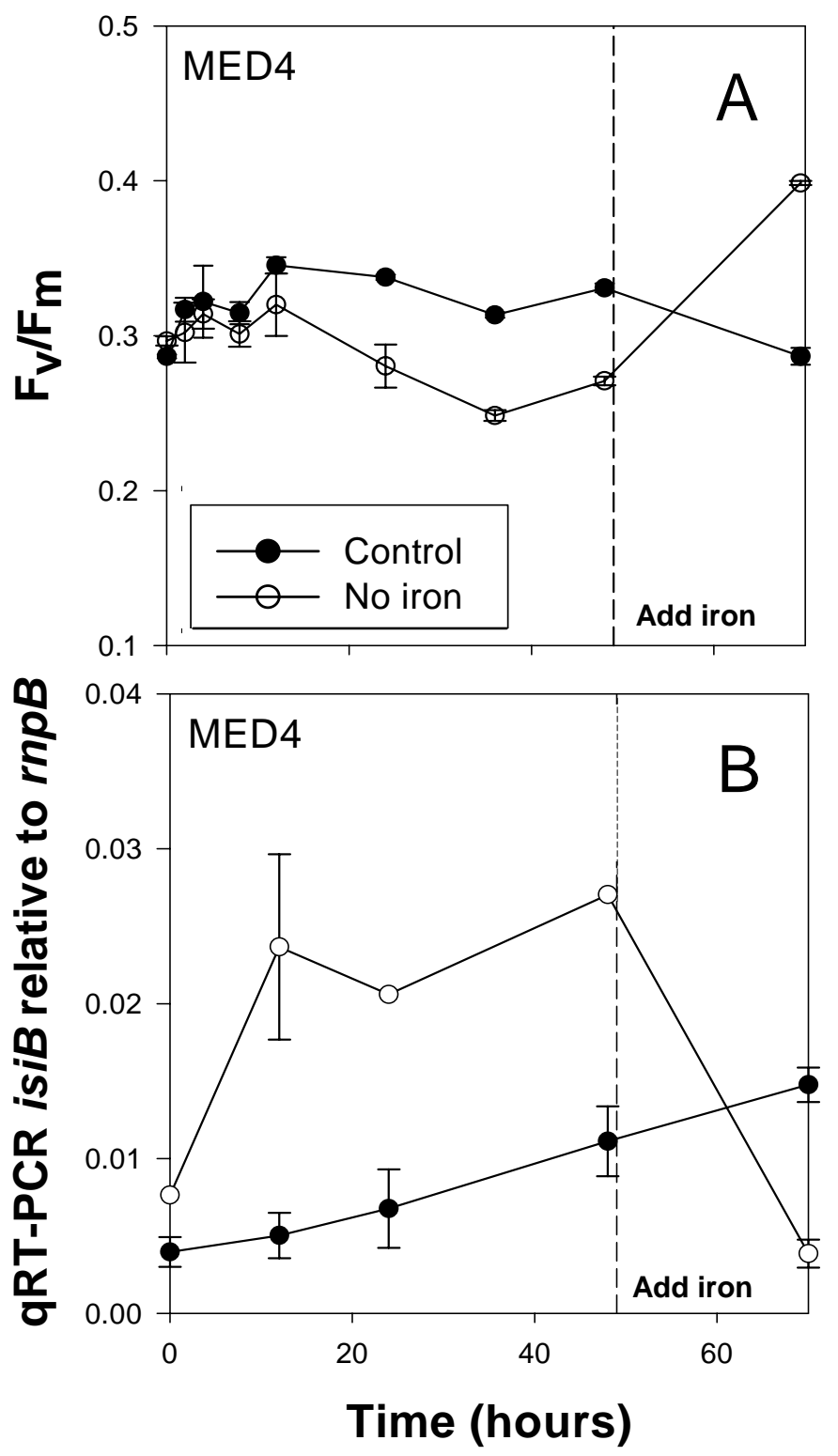

Figure 3: MED4 photochemical efficiency and isiB expression during iron stress. (A) MED4 photochemical efficiency $\left(\mathrm{F}_{\mathrm{v}} / \mathrm{F}_{\mathrm{m}}\right)$ in experimental treatment (no iron) compared to the control during iron deprivation and recovery from iron starvation. (B) MED4 flavodoxin (isiB) expression relative to the house-keeping gene $r n p B$ over the time course of iron deprivation and recovery as a test for iron stress. Dotted line indicates iron addition to the experimental (no iron) treatment to match iron concentrations in the control treatment. 


\section{Global expression responses}

We compared genome-wide differences in gene expression between an iron-deprived treatment $(-\mathrm{Fe})$ and iron-replete control $(+\mathrm{Fe})$ for each time-point of iron deprivation $(0,12,24$, 48, and 70 hours) using DNA microarrays in the Prochlorococcus strains MED4 and MIT9313. We also examined gene expression in response to rescue from iron starvation by comparing control expression at 70 hours $\left(70_{\mathrm{Fe}}\right)$ to expression in the experimental treatment at 70 hours $\left(70{ }_{-\mathrm{Fe}}\right)$, to which iron was added at 49 hours. In both strains, slightly more genes were upregulated than down-regulated in response to iron starvation (Figure 4a). Also, a greater proportion of the MED4 genome responded to iron starvation and rescue than the MIT9313 genome (Figure $4 \mathrm{~b}$ ). During nitrogen starvation, we also see proportionally more of the MED4 genome responding to stress than the MIT9313 genome (Tolonen et al, 2006). Yet, when starved of phosphorus, proportionally more of the MIT9313 genome responds than the MED4 genome (Martiny et al, 2006).

About equal numbers of hierarchical clusters were up- or down-regulated in both strains

(Figure 5). We were particularly impressed by the dynamic changes in gene expression that occur in both MED4 and MIT9313 while the iron-starved cells maintain a maximal growth rate (Figure 1). Several of the clusters contain genes of known-, or predicted- function and these are of particular interest as they will inform the function of uncharacterized differentially-expressed genes (Figure 5). Intriguingly, the known iron-related genes flavodoxin (isiB) and a homolog a component of an iron $\mathrm{ABC}$ transporter (afuA/idiA) clustered together among MIT9313 genes but clustered separately in the MED4 experiment suggesting different roles, or a different regulatory pattern, of these genes in MED4 and MIT9313. Also notable is the observation that after iron addition, at 70 hours, gene expression in MIT9313 between experimental and control treatments is very similar with $\log _{2}$ (fold change) close to zero. In contrast, at the final time-point in MED4 cultures, relative expression between experimental and control treatments is the opposite of what it was during iron starvation. We surmise that at 70 hours, after iron has been added back to the iron-starved cultures, the MED4 control becomes more iron-stressed than the now iron-replete experimental cultures. We hypothesize that the greater cell density in the MED4 cultures (Figure 1) brought about a "blown-buffer" scenario where free $\mathrm{Fe}^{3+}$ is taken up from the media by cells more quickly than equilibrium can be re-established by the EDTA buffer, thus diminishing the pool of available iron. 

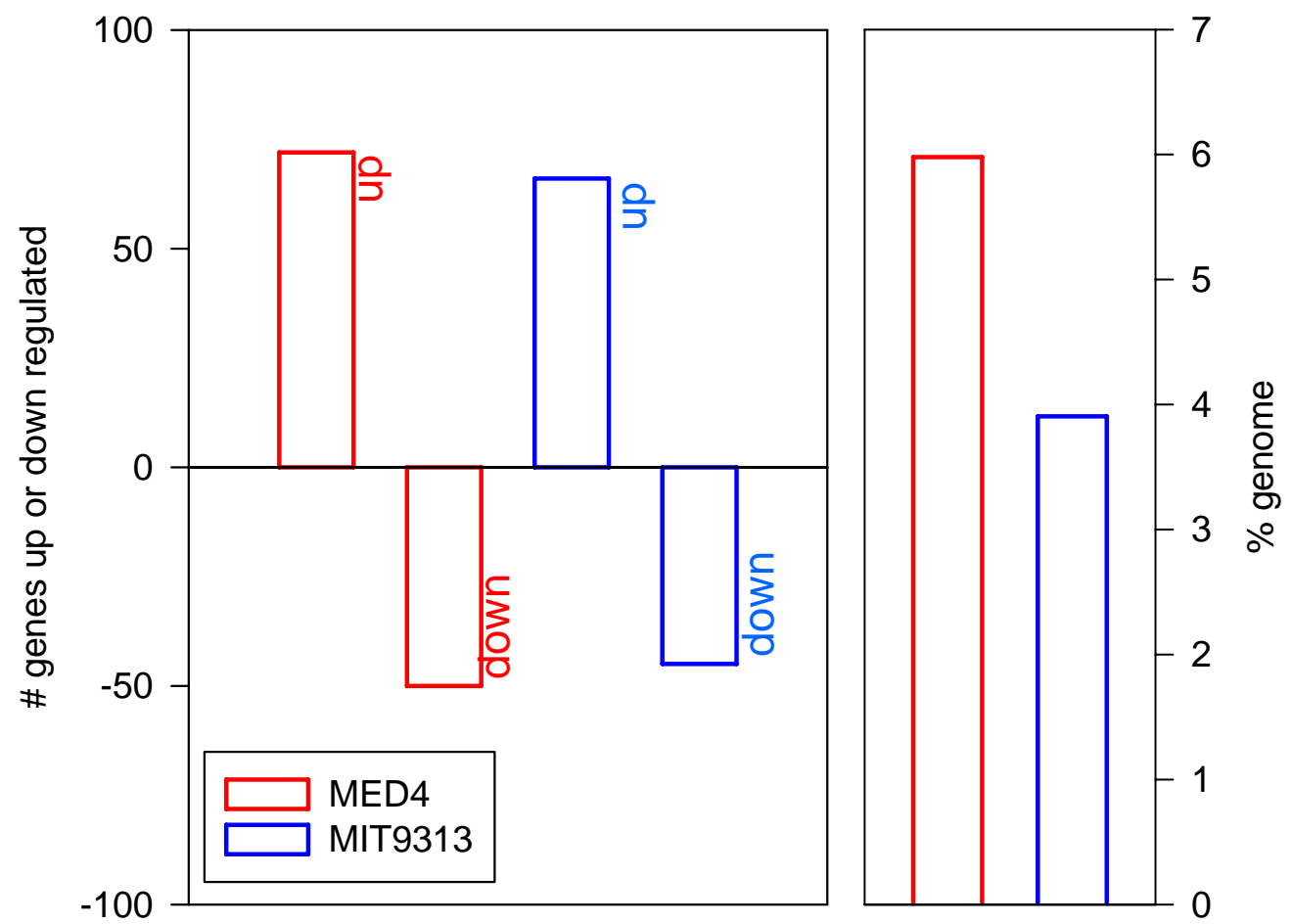

Figure 4: Global expression response of Prochlorococcus MED4 and MIT9313 to iron deprivation and recovery from iron starvation. Differentially-expressed genes have a q-value $<0.01$ (false discovery rate) and $\log 2$ (fold change) greater than 1 or less than -1 in at least one time-point of iron deprivation or in the experimental treatment before and after iron rescue. (A) Number of genes up- or down- regulated in response to iron starvation in MED4 and MIT9313. (B) Percent of genes in MED4 or MIT9313 genomes that respond to iron. 

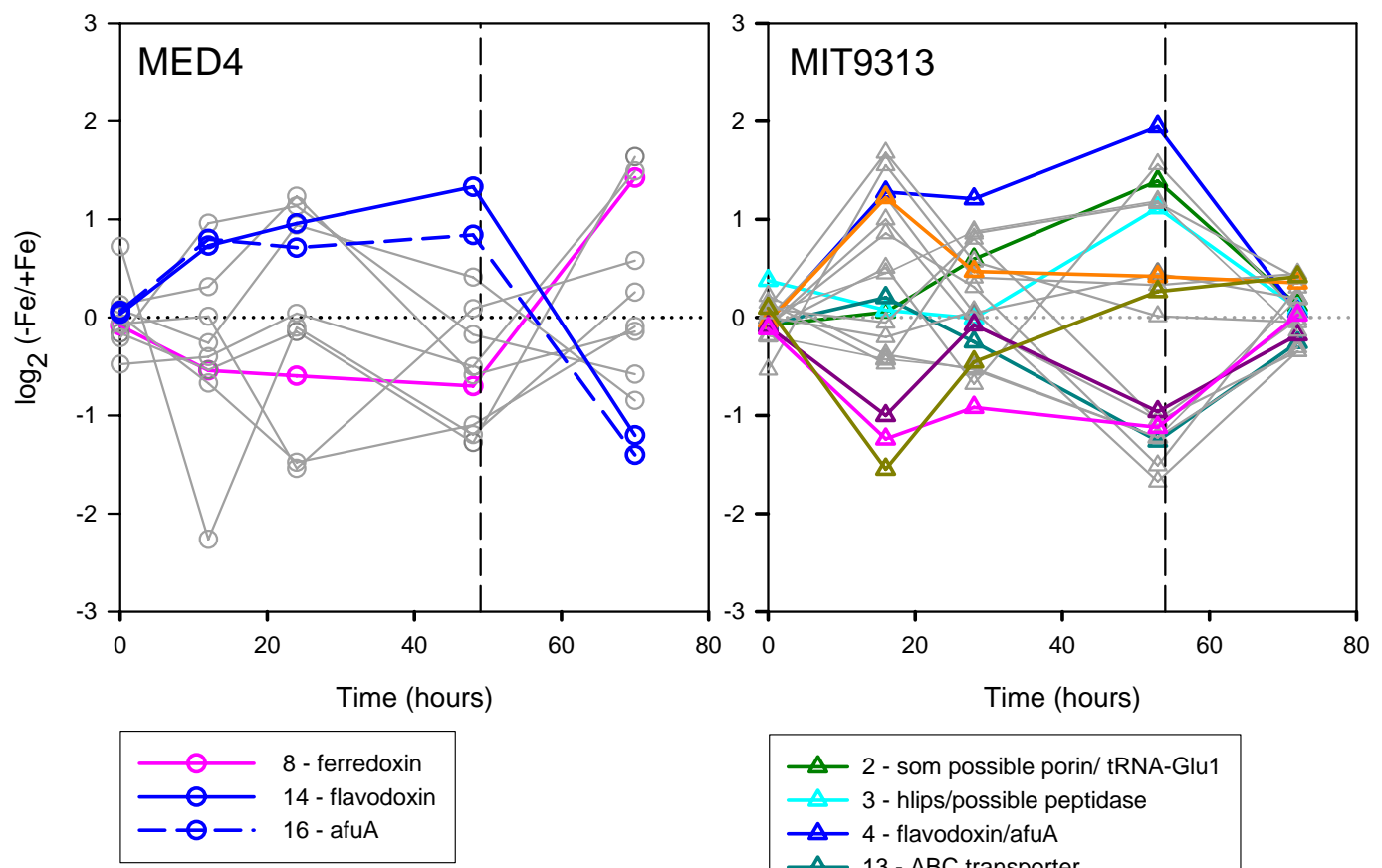

$\Delta-2$ - som possible porin/ tRNA-Glu1
$-\Delta-3$ - hlips/possible peptidase
$\Delta-4$ - flavodoxin/afuA
$\Delta-13$ - ABC transporter
$-\Delta-23$ - ferritin
$\Delta-27$ - glycosyl transferase
$\Delta-30$ - ferredoxin
$\Delta-35$ - tRNA Asn1/Leu1

Figure 5: Mean expression profiles of differentially-expressed genes in each cluster. Cluster profiles are colored if they contain genes predicted to be involved in iron metabolism in Prochlorococcus. Dotted line indicates iron addition to the experimental (no iron) treatment to match iron concentrations in the control treatment. 


\section{Comparison of MED4-MIT9313 ortholog expression in response to iron}

Orthologs differentially-expressed in both MED4 and MIT9313

Out of the more than 1200 genes that are MED4 - MIT9313 orthologs, only three were differentially-expressed in both strains during iron starvation and recovery (Figure 6). These include the classic iron-stress indicator genes, ferredoxin (petF) and flavodoxin (isiB). Ferredoxin is an electron transfer protein associated with PSI that contains 2-3 atoms of iron and cyanobacteria are known to down-regulate ferredoxin in favor of the iron-free electron transfer protein, flavodoxin, during iron stress (Jordan et al, 2001; Rogers, 1987; Straus, 1994). This is evident in our experiments as ferredoxin is down-regulated and flavodoxin is up-regulated over the time-course of iron starvation in both strains (Figure 7). When we compare the rescue response of the experimental treatment in MED4 and MIT9313, flavodoxin expression is downregulated upon iron addition and ferredoxin expression is up-regulated (Figure 6), exactly as one would expect.

The third MED4-MIT9313 ortholog that is differentially-expressed in MED4 and MIT9313 is PMM1164/PMT0287, which is homologous to the proteins afuA in Synechococcus PCC7942 and idiA in Synechococcus PCC6301 (Figure 6) (Alm et al, 2005; Michel, 1999, 2001). We will refer to this gene and protein as afuA/idiA. afuA/idiA is up-regulated at several timepoints in the iron starvation time-course and is down-regulated upon iron rescue in both MED4 and MIT9313 (Figure 7). Homologs of this gene have been extensively studied in other cyanobacteria and it is well known to be up-regulated at the transcript and protein levels during iron deprivation (Katoh et al, 2001; Singh et al, 2003; Webb et al, 2001). Yet, despite its ubiquity among marine cyanobacteria and its reliable response to iron stress in many studies, the protein varies significantly in its size and its localization to either the periplasm (PCC6803 and WH7803) or thylakoid membranes (PCC6301) (Katoh et al, 2000; Webb et al, 2001). The closest relative to Prochlorococcus in which localization of afuA/idiA has been examined is in open-ocean Synechococcus strain WH7803 where the protein appears present in the outer-membrane (Webb et al, 2001). In MIT9313, afuA/idiA clusters with flavodoxin (isiB) but clusters apart from isiB in MED4 (though the pattern of expression is still very similar). This suggests that there may be different functional roles or regulatory pathways for the protein in each strain. In the future, it will be interesting to test whether afuA/idiA in Prochlorococcus responds to Mn-stress (suggesting a role at the thylakoid in collecting Mn atoms for the photosystem II complex) and if the protein is located in different areas of the cell in MED4 and MIT9313. Thus, there is a major role for 


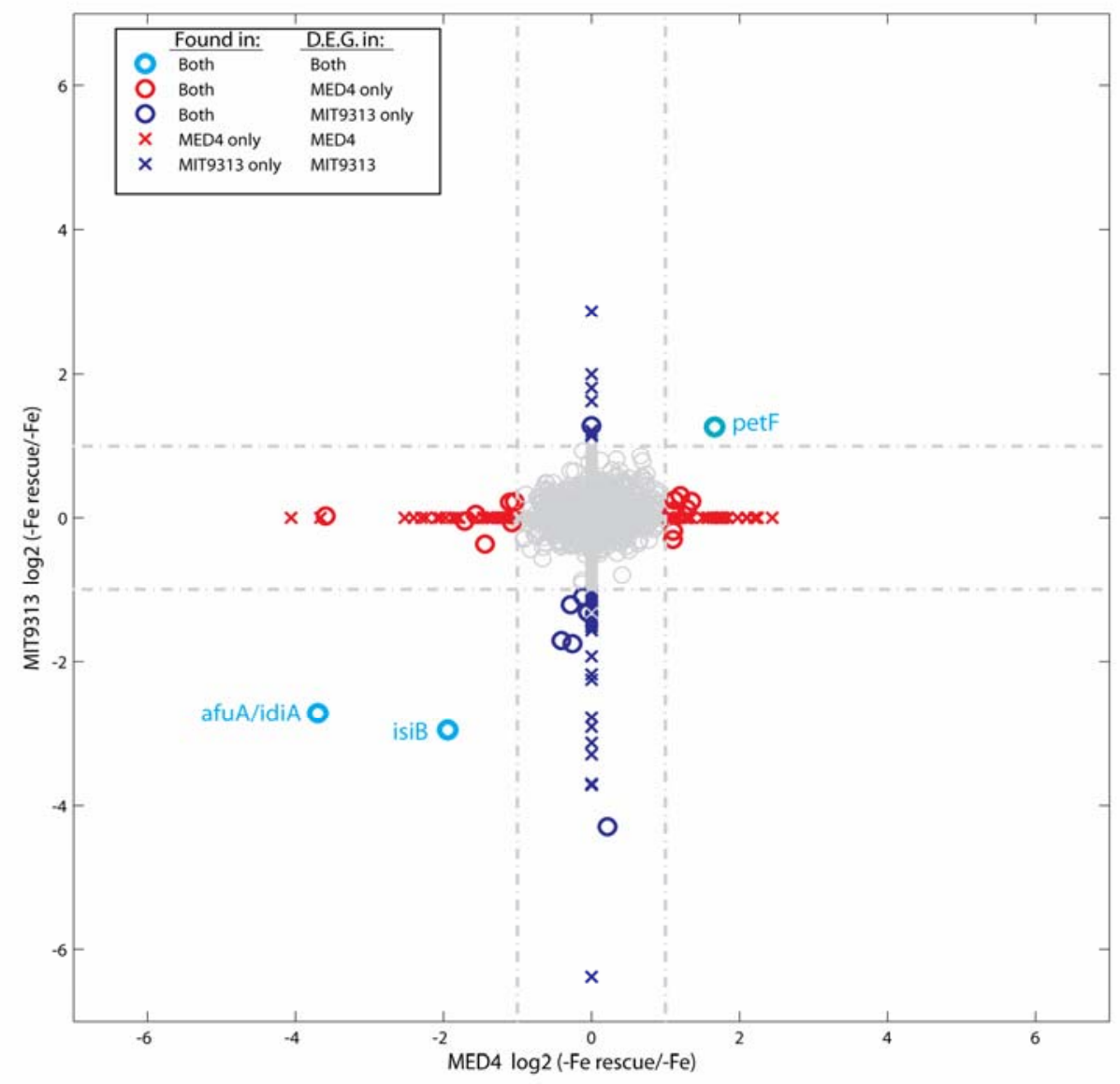

Figure 6: Expression of MED4-MIT9313 orthologs and non-orthologs that are differentially expressed (D.E.G.) in iron recovery in the experimental treatment at 70 hours (- Fe rescue) relative to iron starvation in the experimental treatment at 48 hours (-Fe). Cyan circles represent MED4-MIT9313 orthologs that are D.E.G. in both MED4 and MIT9313. Red circles represent orthologs that are D.E.G. only in MED4. Blue circles represent orthologs that are D.E.G. only in MIT9313. Red Xs represent MED4 genes not found in MIT9313 (non-orthologs) that are D.E.G. in MED4. Blue Xs represent MIT9313 genes not found in MED4 (non-orthologs) that are D.E.G. in MIT9313. Gray symbols represent genes that are not significantly differentially expressed and may represent either MED4-MIT9313 orthologs (circles) or, non-orthologs of either strain (Xs). Genes that are non-orthologs are plotted with values equal to zero in the strain where they are not found. Dashed lines mark a log2(fold change) of 1 and -1, which is the threshold for determining significance along with q-value (false discovery rate) of less than 0.01. petF encodes ferredoxin, an iron-requiring electron transfer protein associated with PSI. isiB encodes flavodoxin, an iron-free electron transfer protein known to substitute for petF under iron-starvation conditions. afuA/idiA encodes a possible periplasmic iron-binding protein involved in iron transport. 

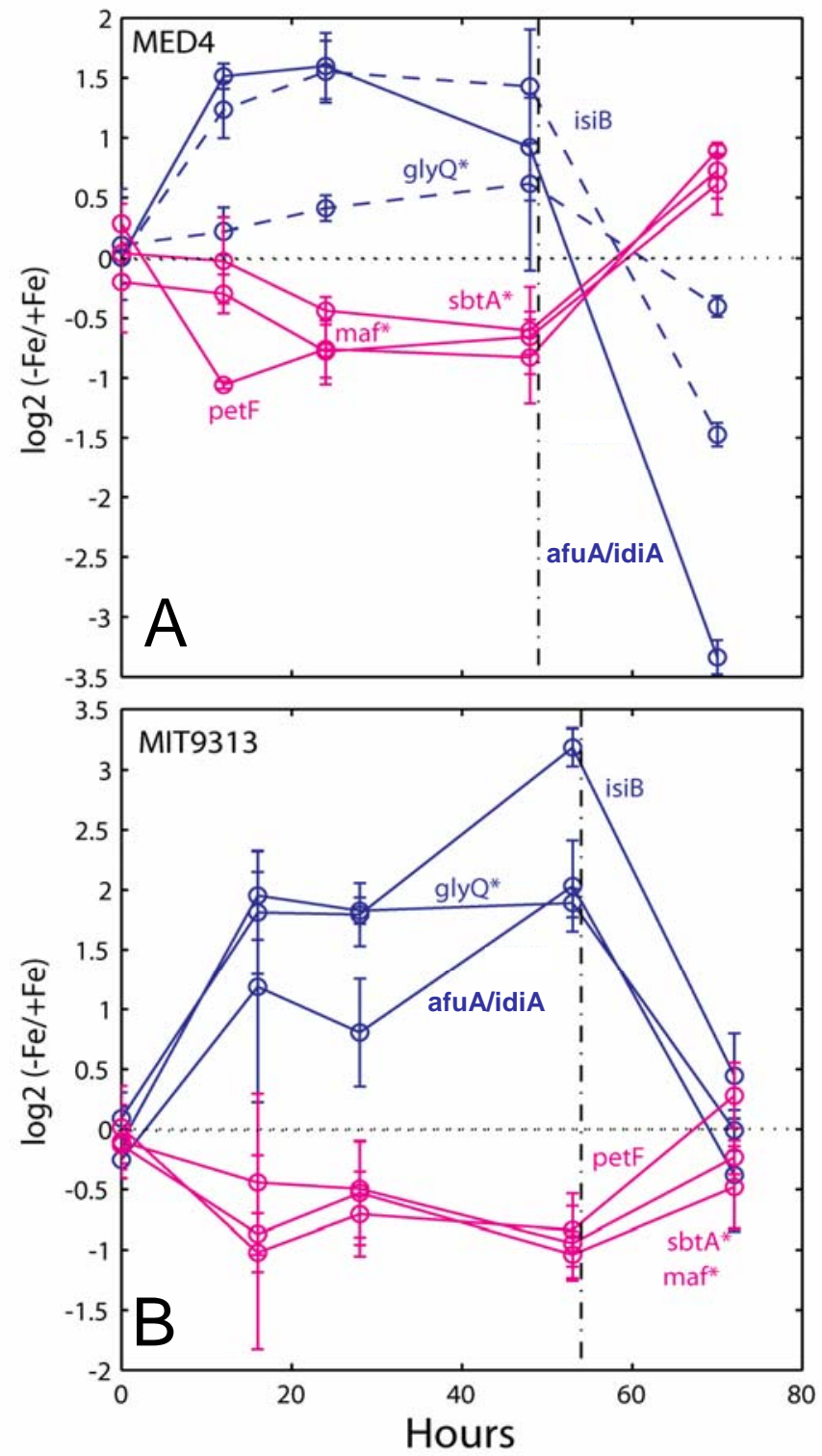

Figure 7: Expression profile of MED4-MIT9313 orthologs that are differentially expressed in both MED4 and MIT9313 during iron starvation and recovery from iron starvation. Color and line texture indicate the cluster to which each gene belongs following the color assigned to clusters in Figure 5. Flavodoxin (isiB) is the iron-free replacement to the electron transfer protein, ferredoxin (petF). afuA/idiA is a homolog to the iron-deficiency induced protein, idiA, that may be involved in iron transport. * Indicates that the gene is only marginally differentially expressed in one or both of the strains (see text for detail). Dotted line indicates iron addition to the experimental (no iron) treatment to match iron concentrations in the control treatment. 
afuA/idiA in the iron metabolism of Prochlorococcus, yet its specific function, whether aiding light-harvesting or facilitating iron transport, remains unclear.

Another MED4-MIT9313 ortholog of note is sbtA (PMM0213/PMT1213), which is a putative sodium-dependent bicarbonate transporter. In both strains, $s b t A$ is differentially expressed just below the fold change threshold we have used to identify differentially-expressed genes, with q-values less than 0.01 but $\log _{2}$ (fold change) of 0.92 in the MED4 experimental treatment after iron rescue, and -0.95 during iron starvation in MIT9313 (Figure 7). As sbtA is implicated in bicarbonate uptake, its down-regulation indicates slowing of carbon fixation by both MED4 and MIT9313 as a result of iron stress (Dufresne et al, 2003).

Several MED4-MIT9313 orthologs were differentially expressed in one strain but were either only marginally differentially-expressed in other strain or not differentially-expressed at all in the other strain (Figure 6, Table 2 and 4). Many of these orthologs are present in two regions of the MED4 and MIT9313 genomes that are enriched in predicted iron-related genes and include the afuA/idiA region (Figure 12) and the futC region.

GlyQ is a class II aminoacyl-tRNA synthetase, PMM1165/ PMT0283, and is part of the Prochlorococcus/Synechococcus core genome as defined in Kettler et al. 2007. It is downregulated upon iron rescue in MIT9313 and only marginally down-regulated in MED4. In MED4, glyQ is directly up-stream of afuA/idiA and just a few genes down-stream of isiB while in MIT9313, glyQ is separated from afuA/idiA by four genes, two of which (PMT0284 and PMT0286) respond to iron-deprivation in MIT9313 (Figure 12). In fact, this region is predicted to be involved in iron uptake by its proximity to isiB and $a f u A / i d i A$ as described in Coleman and Chisholm (2007), so is of interest. A maf-like protein (PMM1159/PMT1181) is another ortholog that responds to iron addition in MIT9313 but is only marginally responsive to iron in MED4 (Table 3). In MED4, maf is in the afuA/idiA region (out of range in Figure 12), though, in MIT9313, the location of maf does not imply involvement in iron metabolism. The maf-like protein in Prochlorococcus is about 30\% similar to maf genes in E.coli and Bacillus subtilis (Butler et al, 1993; Levin et al, 1992; Minasov et al, 2000). Early work on Maf indicated a role in the inhibition of septum formation in B. subtilis (Butler et al, 1993). While the function of this conserved family of proteins is still unknown, more recently, the crystal structure of the $B$. subtilis Maf protein has been solved and shows structural similarity to the C-terminal anticodonbinding domain of glycyl-tRNA synthetase (also differentially expressed in response to iron, see above), three other aminoacyl-tRNA synthetases as well as nucleotide or RNA-binding proteins 
(Minasov et al, 2000). Also, the N-terminal region of Maf in B. subtilis shares features with $\mathrm{C}$ terminal region of the RNAse $\mathrm{H}$ of E. coli (Minasov et al, 2000). Taken together, these observations suggest that Maf plays a role in the recognition or processing of nucleotides or oliognucleotides and may play a general role in DNA repair (Minasov et al, 2000). Minasov et al. 2000 also point out that Maf and RNAse $\mathrm{H}$ have a conserved aspartate residue that is known to be involved the metal-ion dependent hydrolysis of oligoribonucleotides (Minasov et al, 2000), which could explain the positive response of the maf-like protein to iron addition in this experiment (Figure 7).

Orthologs differentially expressed in either MED4 or MIT9313

A possible porin, PMT0284, is another iron-stress induced gene in the afuA/idiA region of MIT9313 that has an ortholog in MED4 (yet, in a different region of the genome) where it is not differentially expressed in response to iron availability (Figure 12). The ortholog in MED4, PMM0709, is annotated as a phoE, a possible porin, involved in orthophosphate acquisition and is up-regulated during phosphate stress in MED4 (Martiny et al, 2006). In MIT9313, the gene is not up-regulated during phosphate starvation.

MED4-MIT9313 orthologs in close proximity to the predicted iron ABC transporter component, futC, are also active during iron stress and recovery but none of them are differentially-expressed in both strains. For instance, in MIT9313, which has two copies of ferritin, one copy (PMT0499) is up-regulated at 12-hours after the initiation of iron starvation and is one gene down-stream of futC. MED4 contains one copy of ferritin (PMM0804), which is an ortholog of PMT0499 and is also down-stream of futC. But in MED4, the ferritin is not differentially-expressed in response to iron. Ferritin is up-regulated during iron-stress in MIT9313, though its typical expression pattern in E. coli involves up-regulation during conditions of excess iron (Andrews et al, 2003). Possibly, in Prochlorococcus, ferritin is involved in moving iron within the cell as proteins, such as the light-harvesting complexes, are re-modeled during iron stress (Busch et al, 2008).

A bacterial regulatory protein of the Crp family (PMM0806) is another very interesting MED4-MIT9313 ortholog in the futC region that is only differentially-expressed in MED4. PMM0806 is strongly up-regulated during iron deprivation but its ortholog in MIT9313, PMT0497, is not differentially-expressed. Though, in MIT9313, it is in close proximity to $p c b B$, one of the most highly iron-responsive up-regulated genes in MIT9313. 


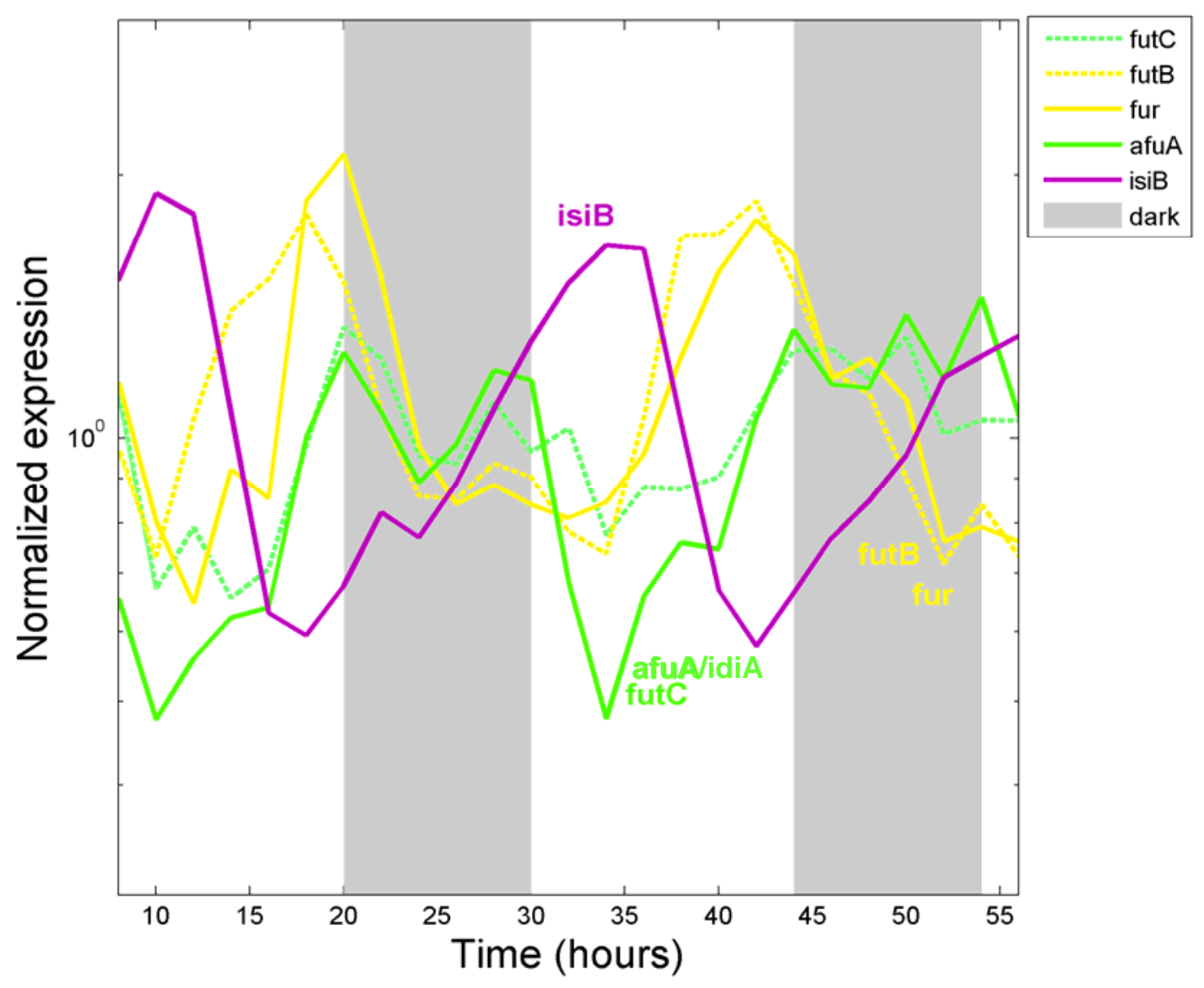

Figure 8: MED4 diel expression of selected Prochlorococcus core genes that are predicted to be related to iron. isiB encodes flavodoxin an iron-free electron transfer protein that replaces the iron-requiring electron transfer protein, ferredoxin, during iron stress. afuA/idiA, futB, and fut $C$ are predicted to be components on an iron-transporting ABC transport system. Fur is the ferric uptake regulator. Expression data is published in Zinser et al. 2009. Genes are colored by their cluster membership in the diel expression experiment. Clusters 1-16 contain genes that are periodic in expression over the diel cycle. Cluster 17 contains genes that have aperiodic expression profiles. Cluster 18 contains genes that appear not to be expressed above a background threshold defined in Zinser et al. 2009. Dark shading indicates periods of darkness during the two diel cycles that are presented here. 
A gene encoding pentapeptide repeats (PMM1350/PMT1554) was another very highly up-regulated MIT9313 gene but was not differentially-expressed in MED4. Interestingly, the MED4 genome contains two copies of the pentapeptide repeat gene and MIT9313 contains three copies though only one copy responds to iron stress. Under conditions of diel light availability, MED4's pentapeptide repeat gene (PMM1350) is expressed and clusters with isiB as well as genes such as rubredoxin, plastocyanin, glutathione peroxidase, and components of the PSI and PSII complexes suggesting a role for pentapeptide repeats in photosynthesis (Zinser et al, 2009). Among MIT9313 genes, the gene for pentapeptide repeats (PMT1554) clusters with pcbB during iron starvation, which is known to form a super-complex with PSI to enhance light harvesting efficiency during iron stress (Bibby et al, 2003). Pentapeptide repeats are common in the genomes of other organisms where they are annotated as low complexity proteins and in the case of Leptospira is annotated as involved in Fe-S cluster assembly with $\sim 30 \%$ sequence homology to PMT1554. Our results suggest a role for Prochlorococcus pentapeptide repeats similar to that of pcbB in MIT9313, but given what little is known about pentapeptide repeats, its function in Prochlorococcus is unclear.

\section{Iron-related core genes that were not differentially expressed in response to iron}

Beyond afuA/idiA, isiB, and petF, there are several other genes in the Prochlorococcus core genome that are predicted to be involved in iron metabolism but surprisingly did not respond to iron availability in this study. Possibly, we did not impose severe enough iron stress to cause differential expression of these iron-related genes. Alternatively, the genes may be regulated posttranscriptionally, which could mask the evidence of their involvement in the iron-stress response in a transcriptional study like ours. Though these two questions are beyond the scope of our study, by examining MED4 whole-genome expression over the diel cycle (Zinser et al, 2009) we were able to ask whether these predicted iron-related genes are expressed in iron-replete cultures growing under laboratory conditions and how their expression patterns relate to light availability and the expression of other MED4 genes.

One possible iron transport system in Prochlorococcus is the futABC ABC-transporter system which is encoded in the Prochlorococcus core genome. Intriguingly, some proteins that work with the futABC transport system, such as a mechanism to provide energy to the outermembrane iron transporters usually encoded by ton $B$ and exbD, have not been recognized in the Prochlorococcus genomes (Rocap et al, 2003; Webb et al, 2001). The periplasmic binding 
protein for the futABC system, afuA/idiA (futA), is up-regulated with iron deprivation in both strains (Figure 7). futC, the ATP-binding component of this iron transport system, is downregulated in MED4 following iron addition (not represented in Table 2 because $\log _{2}$ (fold change) is -0.9) but it is not differentially expressed in MIT9313. The permease component of the transporter, futB, is not differentially expressed in either MED4 or MIT9313 experiments.

Over the diel cycle, all three components of the futABC transporter show periodic expression in MED4 (Figure 8 and Appendix B, Figure 19). Thus, there appears to be a role for these proteins in Prochlorococcus metabolism. Furthermore, afuA/idiA and futC cluster together and peak in expression at sunset (though there may be a second peak during the night) and futB clusters with one copy of the ferric uptake regulator (fur) (PMM0637) that is thought to repress transcription of iron acquisition genes when iron is abundant. A second copy of fur (PMM1030) is present in the core genome and clusters with ferritin (an iron storage protein) and though its expression is a-periodic over the diel cycle, it is expressed above the background threshold (defined in Zinser et al. 2009) (Appendix B, Figure 19). It is likely that futABC, the fur genes, and ferritin are involved in Prochlorococcus iron metabolism. The diel cycling of one copy of fur and not the other is intriguing and it will be interesting to learn how their roles in regulating iron metabolism differ (Figure 8).

sufBD (PMM0071 and PMM0073) and sufC (PMM0072) make up another potential iron ABC-transporter in the Prochlorococcus core genome. Yet, they are not differentially-expressed in our iron starvation conditions. In Synechocystis, sufB (slr0074) is down-regulated during iron starvation (Singh et al, 2003). We see that these suf transport components are expressed in the diel experiment in a cyclic pattern, and cluster with several other $\mathrm{ABC}$ transporters and some possible Fe-S oxidoreductases (Appendix B, Figure 19) (Zinser et al, 2009). Based on the expression of sufB in Synechocystis, we hypothesize that these transporters are involved in iron transport when availability is high and may represent a system of low-affinity transport.

We also examined the expression behavior of possible Fe-S proteins over the diel cycle in comparison to known iron-containing proteins like petC (Rieske Fe-S of the cytochrome $\mathrm{b}_{6} / \mathrm{f}$ complex) and psaC (binds three [4Fe-4S] clusters in photosystem I) (Appendix B, Figure 19b). We observe that the Fe-S proteins involved in photosynthetic electron transfer, psaC and petC, peak in expression about mid-day and so we expect that PMM1498, a Fe-S binding protein that follows a similar expression pattern is also involved in photosynthetic electron transfer. Several of the Fe-S proteins peak in expression during the night, including sufE, which is annotated as 
being involved in Fe-S assembly (Appendix B, Figure 19b). Therefore, we hypothesize that the other Fe-S proteins that demonstrate peak expression during the night are also involved in the assembly of Fe-S proteins and include PMM0098, PMM1481, and PMM1560.

Finally, several ferredoxin proteins and ferredoxin-dependent proteins are encoded in the Prochlorococcus core genome but only one, PMM1352 (petF), responds to iron starvation (Figure 7). We observed that other ferredoxins, with the exception of the phycoerythrobilin ferredoxin oxidoreductases ( $p e b A$ and $p e b B$ ), are expressed in MED4. And PMM1352 and PMM1477 were found to be constitutively-expressed over the diel cycle (Zinser et al, 2009). Because PMM1477 (fer) clusters with the iron-responsive PMM1352, it is puzzling why it did not respond to iron starvation as well (Appendix B, Figure 19).

In summary, we observe that while most of the predicted iron-related genes in the Prochlorococcus core genome are expressed in log-phase cultures grown with light-dark cycling, most are not differentially-expressed under our conditions of iron starvation. This is likely due to post-transcriptional regulation or the relatively mild and short-duration (i.e. minimal limitation of growth rate) iron-stress conditions we have imposed in this experiment.

\section{Iron-response of the non-MED4/MIT9313 orthologs}

The genes that are not shared between MED4 and MIT9313 (i.e. non-MED4/MIT9313 orthologs) responded strongly to iron availability suggesting that iron metabolism is a source of diversity among Prochlorococcus ecotypes and an important aspect of the adaptation of Prochlorococcus to local environmental conditions (Figure 6). First, we examined the distribution of the iron-responsive genes among the genomes of all twelve sequenced Prochlorococcus genomes and four Synechococcus. A low percentage of the differentially-expressed genes in either MED4 or MIT9313 belong to the Prochlorococcus core genome or the Prochlorococcus/Synechococcus core genome (Figure 9a). The iron-responsive genes of MED4 are highly-represented among the other high-light adapted strains, which leads us to believe that the mechanisms used to deal with iron stress are, in large part, linked to light physiology or phylogeny rather than iron availability in the environment from which the Prochlorococcus ecotype was isolated (Figure 9b). For example, MIT9515 was isolated from the Equatorial Pacific where Prochlorococcus is known to be iron-limited (Mann et al, 2000), yet, its genome contains similar numbers of MED4 iron-responsive genes as the other high-light adapted ecotypes, which were isolated from less iron-limiting waters. We expect the same result from the clades of low- 


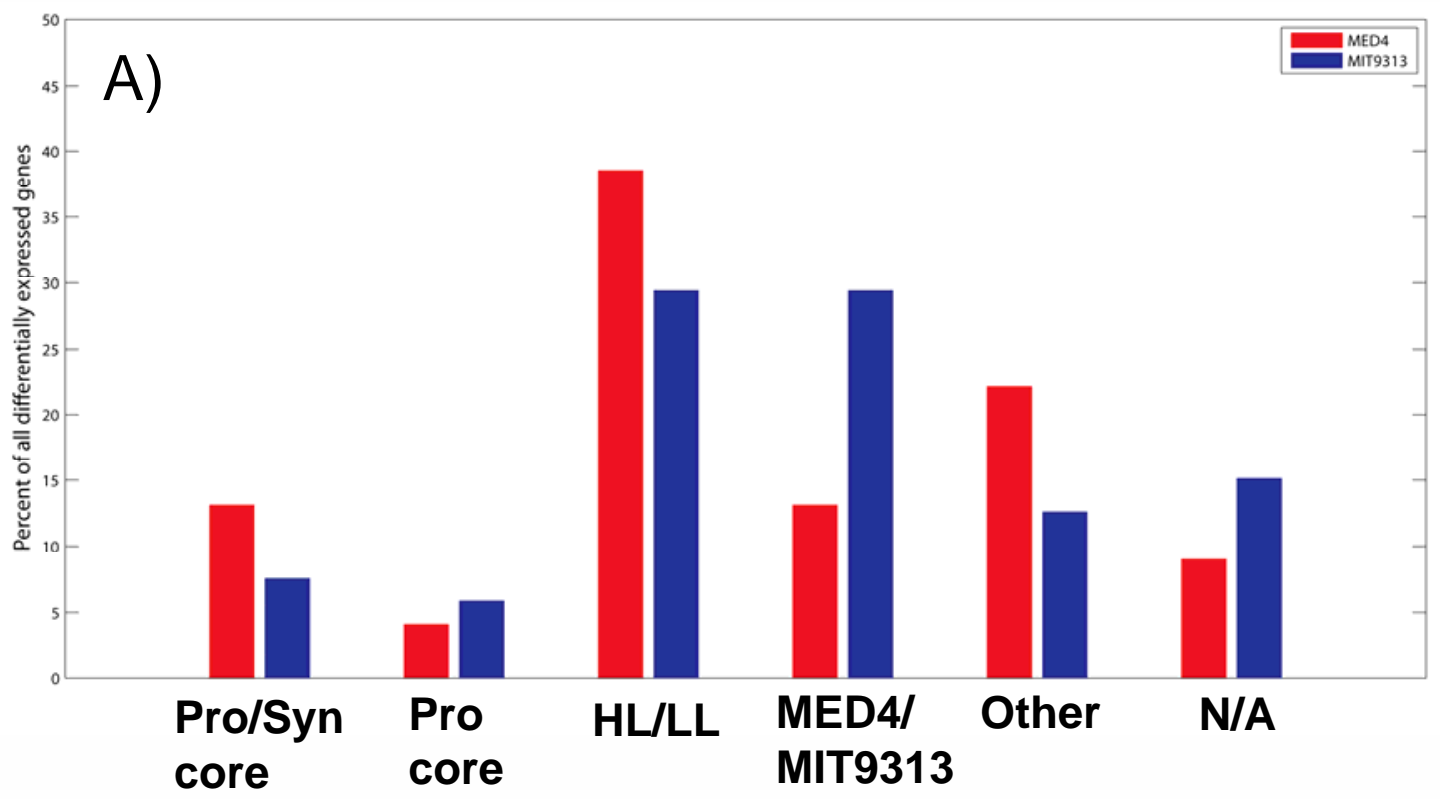

Figure 9: (A) Distribution of all MED4 or MIT9313 iron-responsive genes among the genomes of other Prochlorococcus and Synechococcus. "Pro/Syn core" indicates genes that are present in all Prochlorococcus genomes (12) and the four Synechococcus genomes that were tested in Kettler et al. 2007. "Pro core" indicates genes that are present in all Prochlorococcus genomes and any, but not all, of the Synechococcus genomes. "HL/LL" contains MED4 genes that are present only in other high-light adapted Prochlorococcus or MIT9313 genes that are present only in other low-light adapted Prochlorococcus. "MED4/MIT9313” includes genes that are present only in MED4 or MIT9313. “Other” includes genes that are not isolated to either high-light or low-light adapted genomes but are not part of the core genome. "N/A" contains pseudogenes, small RNAs, and tRNAs to which homology is not assigned in Kettler et al. 2009. Continued next page. 


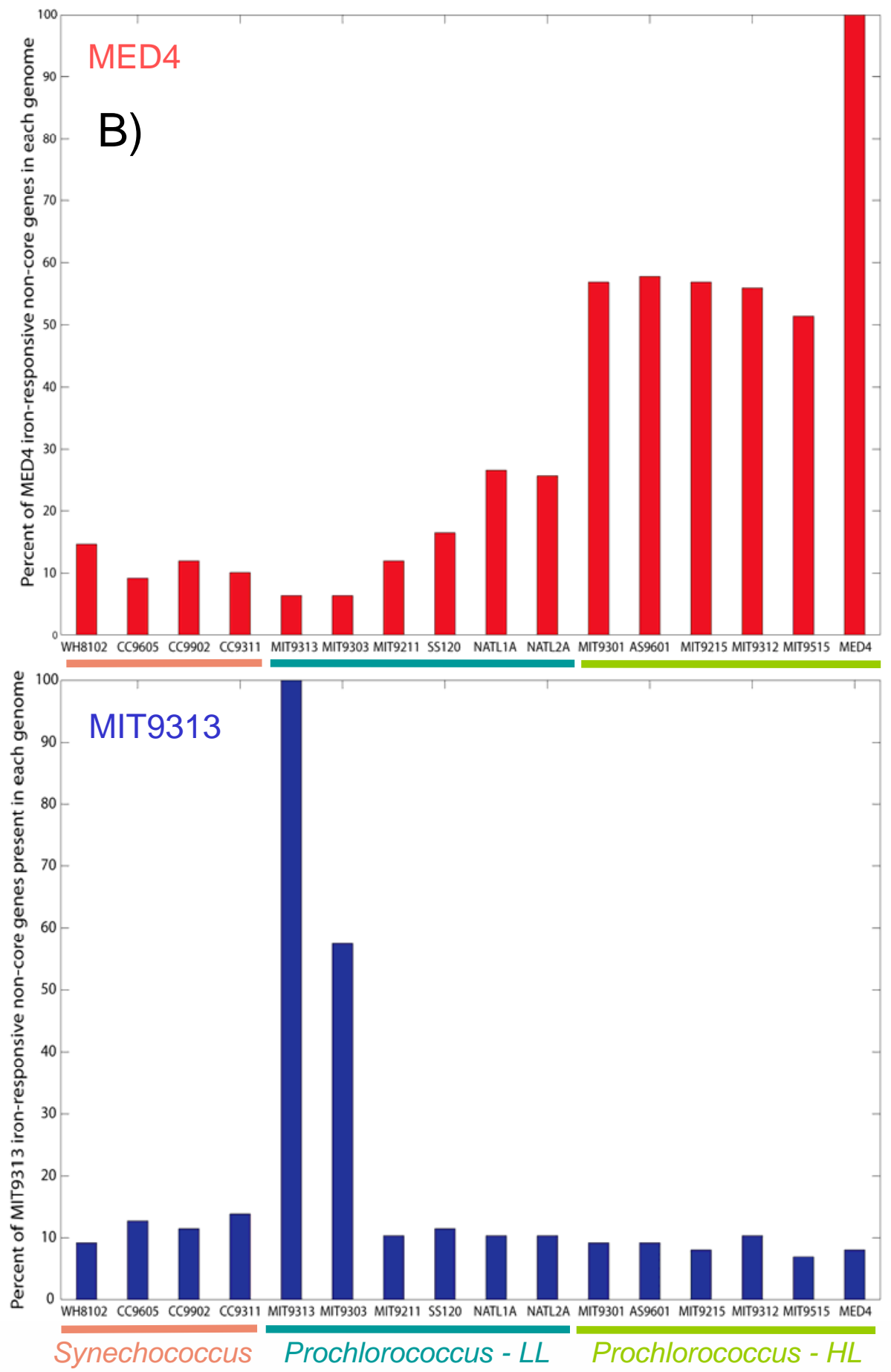

Figure 9, continued: (B) The distribution of iron-responsive non Prochlorococcuscore genes in the other Prochlorococcus and Synechococcus genomes. These genes include all genes from Figure 9a with the exception of genes in the Pro/Syn core and Pro-core categories. Genomes are organized along the x-axis according to phylogeny and the $y$-axis represents the percentage of either MED4 or MIT9313 iron-responsive genes that are present in each genome. 


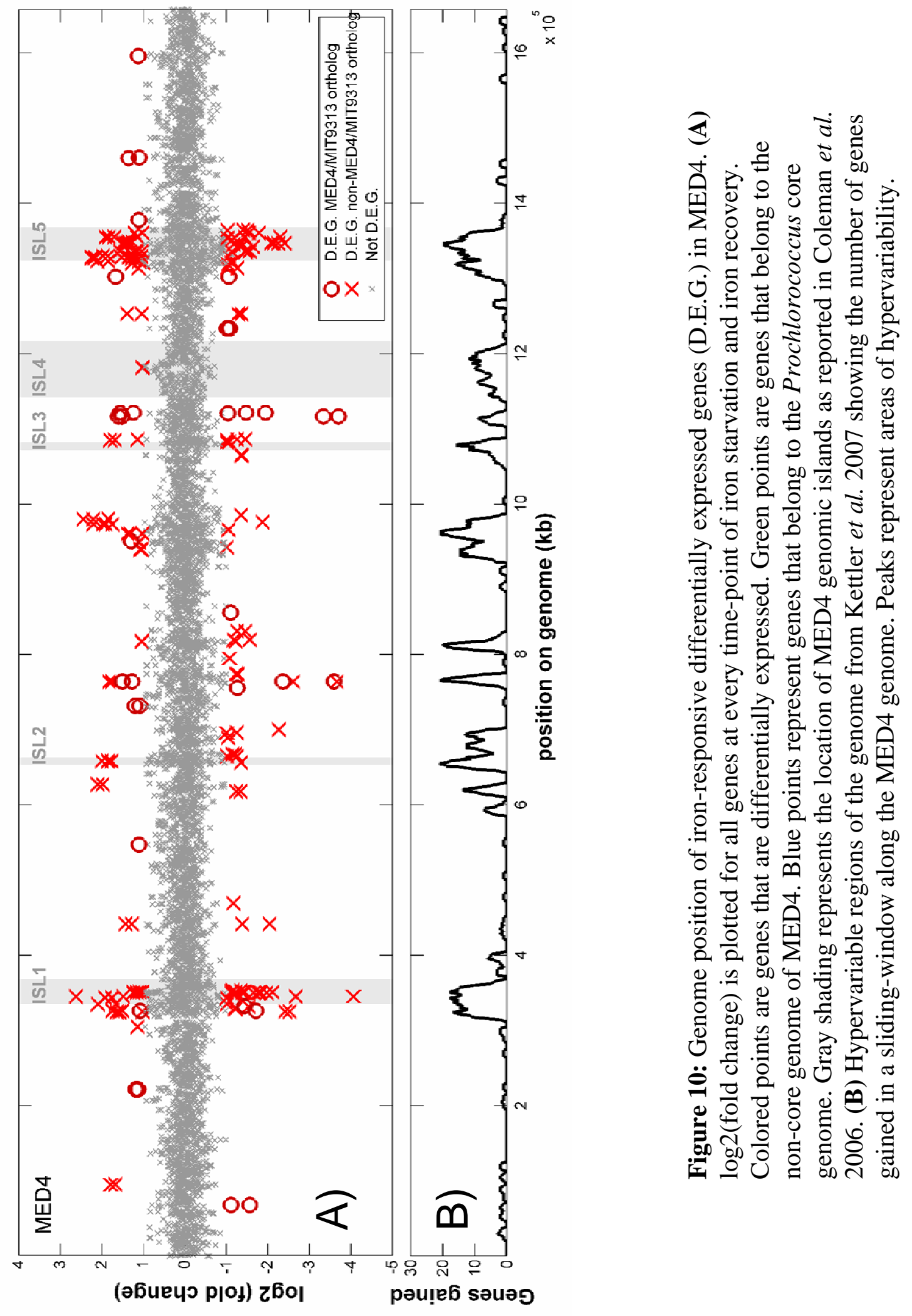




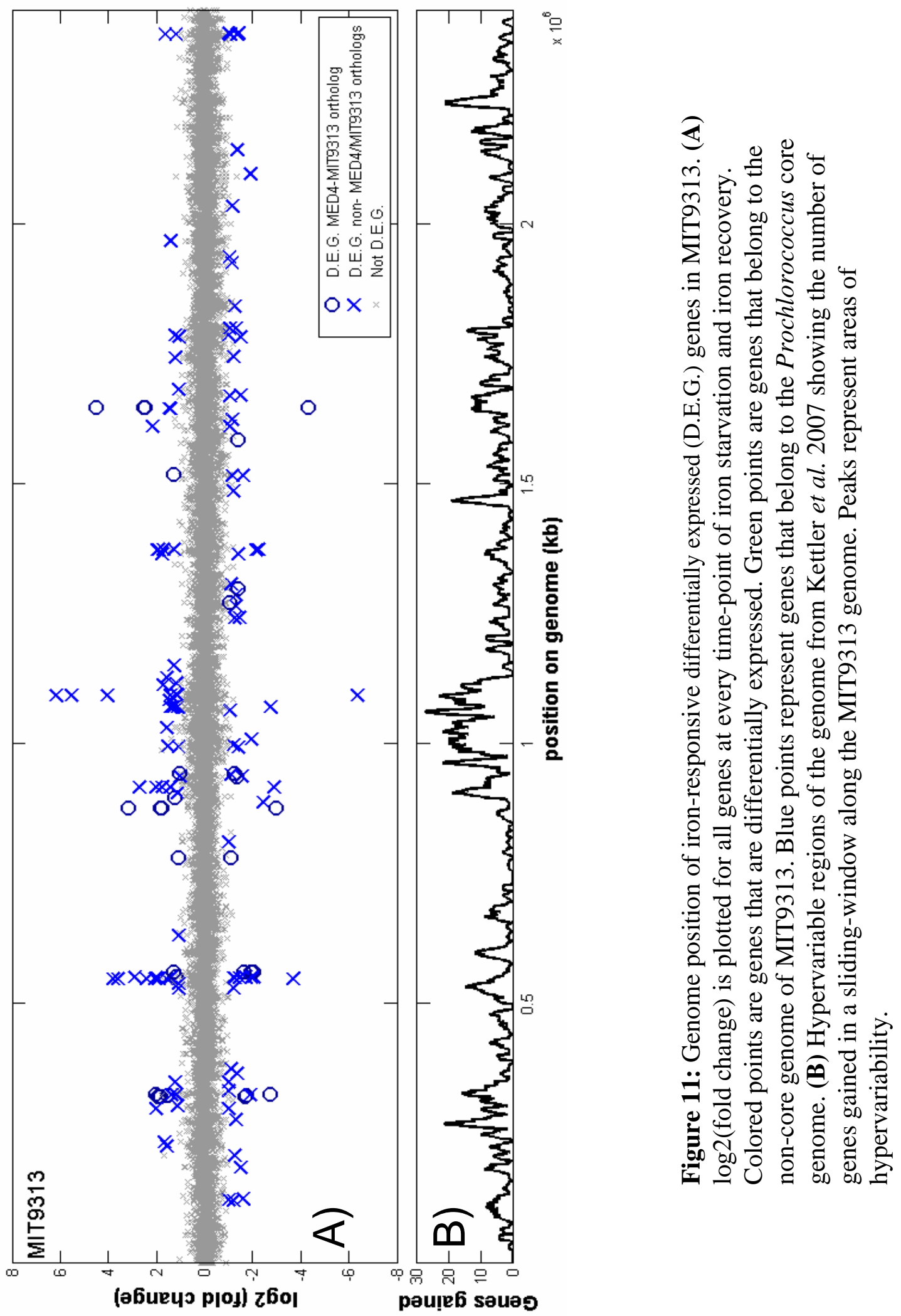




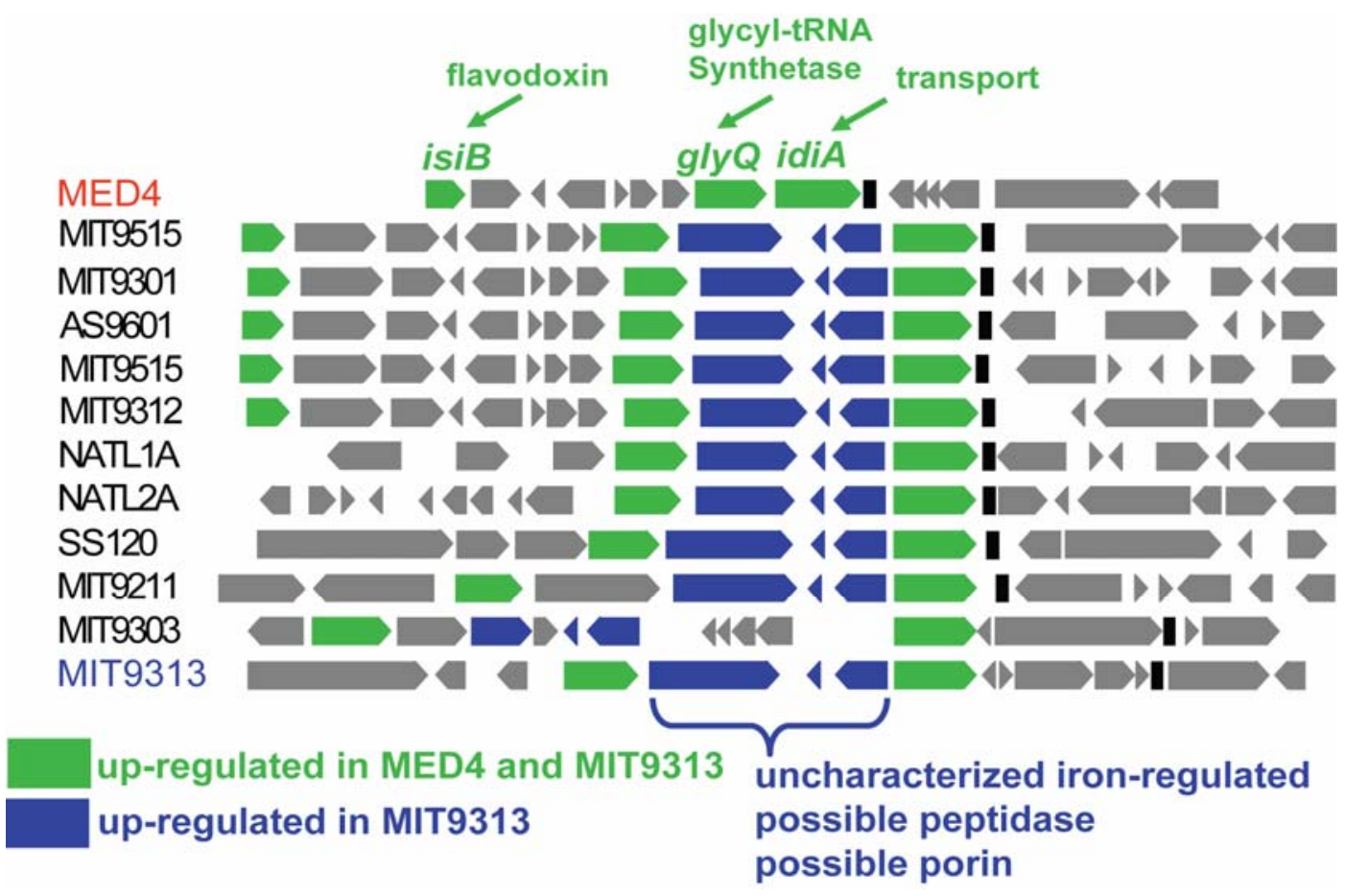

Figure 12: Genome organization of the afuA/idiA region among sequenced Prochlorococcus isolates. Genes represented in green are up-regulated in both MED4 and MIT9313. Genes represented in blue are up-regulated in only MIT9313. Genomes are listed in order of 16S/23S ITS phylogeny as published in Kettler et al. 2007. 
light adapted Prochlorococcus, yet the higher diversity among the low-light adapted sequenced ecotypes compared to the high-light adapted sequenced ecotypes (Kettler et al, 2007) makes it impossible to observe at this time.

Next we examined the position of iron-responsive genes along the genomes of MED4 and MIT9313 (Figure 10 and 11). As expected, the differentially expressed non-MED4/MIT9313 orthologs of both strains gathered in the hypervariable regions of the genomes and the orthologs were scattered across the entire genome. Interestingly, the confinement of non-orthologs to hypervariable regions is more pronounced in MED4 than MIT9313 and is probably because the islands are less well defined among low-light ecotypes (Coleman et al, 2006; Kettler et al, 2007). In MED4, iron-responsive genes were present mostly in ISL1, ISL2, and ISL5 as well as a hypervariable region between about 900,000 and 1,000,000 kb along the genome. Hli21 is present in MED4 ISL2 though hli4, 16, and 17 are not in genomic islands they do coincide with regions of the genome where many genes have been gained (Kettler et al, 2007). All other strongly differentially-expressed genes unique to MED4 are present in MED4's genomic islands as defined in Coleman et al. 2006. These include PMM1229 which is part of a two-gene operon that encodes the dehydrogenase E1 (2-oxoglutarate dehydrogenase) and is present in ISL4 in MED4 and is up-regulated following iron addition. The adjacent genes, PMM1230 and PMM1231 are marginally differentially expressed as well and have no known function. Notably, relatively few genes are differentially expressed in ISL4 compared to the other island regions. This is likely because ISL4 contains many genes that are involved in cell surface modification that do not depend on iron availability (Coleman et al, 2006). MED4 ISL5 contains PMM1391 which is unique to MED4 and encodes a possible helix-turn-helix protein that could be involved in regulation.

Most of the iron-responsive genes that are unique to MIT9313 are also of unknown function, though a few have enough homology to other organisms to assign putative function. PMT0328 is not well characterized but is described as containing a possible bromodomain next to a zinc-finger domain and is down-regulated during iron starvation in MIT9313. PMT0842 is annotated as a pyrimidine dimer DNA glycosylase so may be involved in repairing DNA damage that is caused by iron-stress as it is up-regulated during iron deprivation. This DNA repair enzyme could give MIT9313 an advantage over MED4 in dealing with the oxidative stress that accompanies iron stress and explain the ability of MIT9313 to grow at lower iron concentrations than MED4 (Chapter 2). PMT0107, PMT0108, and PMT0110 are also unique to MIT9313 and 
are down-regulated during iron starvation. PMT0108 encodes the ATP-binding component of an $\mathrm{ABC}$ transporter with about $\sim 40 \%$ homology to polysaccharide $\mathrm{ABC}$ transporters in Burkholderia. PMT0107 and PMT0110 are conserved hypothetical proteins.

Several iron-responsive genes in MED4 and MIT9313 are present in other Prochlorococcus genomes but are not strictly associated with either the high-light adapted clades or low-light adapted clades. For example, PMT0496 in MIT9313 encodes the chlorophyll-binding protein $(p c b B)$ which is not present in MED4 but is present in many other Prochlorococcus strains. All Prochlorococcus contain at least one copy of $p c b$ and sometimes multiple copies. The $p c b$ in MED4 ( $p c b A)$ is not an ortholog to PMT0496 and does not respond to iron. In MIT9313, $p c b B$ is one of the most highly up-regulated genes during iron-stress and clusters with other known iron-related genes such as afuA/idiA and flavodoxin (isiB). Our finding is supported by Bibby et al. 2003 where there is no change in MED4 or MIT9313 pcbA expression during iron starvation but there is up-regulation of MIT9313 pcbB. Bibby et al. 2003 showed that $p c b B$ associates with PSI during iron stress in MIT9313 which is similar to the formation of a 18-mer IsiA-PSI supercomplex that is induced under iron-limiting conditions in Synechococcus PCC7942 and appears to compensate for the reduced PSI:PSII ratio in that organism during iron stress (Bibby et al, 2001; Boekema et al, 2001). The Pcb-PSI supercomplex observed in Prochlorococcus MIT9313 is absent in MED4 during either iron-replete or iron-limiting conditions but both strains produce a PcbA-PSII supercomplex that is immune to fluctuations in iron availability (Bibby et al, 2003). We hypothesize that the ability of MIT9313 to enhance light-harvesting efficiency during iron stress may contribute to its ability to survive at lower iron concentrations that MED4.

Other differentially-expressed genes that are not in the core genome and are not confined to the genomes of either high- or low-light adapted ecotypes are PMT0285 and PMT0286. PMT0285 encodes a possible peptidase (family M20/M25/M40) and PMT0286 (piuC) is an uncharacterized iron-regulated protein. Both genes are induced during iron stress and repressed with iron addition. Interestingly, both genes are located near afuA/idiA (PMT0287) in MIT9313. They are present in all other Prochlorococcus genomes but not present in MED4 and are predicted to be involved in iron uptake (Coleman and Chisholm, 2007) (Figure 12). This genome region may important in determining iron physiology among Prochlorococcus ecotypes.

Another interesting iron-responsive non-MED4/MIT9313 ortholog is PMM1032, which is annotated as the substrate-binding protein of a possible Mn ABC transporter (lraI) in MED4. 
IraI is up-regulated during iron stress and abruptly down-regulated with iron addition and does not have an ortholog in MIT9313, though orthologs are present in the genomes of the other highlight adapted strains as well as SS120, NATL1A, and NATL2A. Though, PMT2203 in MIT9313 (also annotated as a $\mathrm{Mn}$ transporter) does display about $\sim 30 \%$ similarity to a small region of IraI. In MED4, IraI is close to a copy of the ferric uptake regulator (fur) and other putative Mn transporters (microbesonline.org). This mode of expression suggests that Prochlorococcus may use manganese as a substitute for iron in certain metalloproteins during iron stress. Physiological studies on the ability of $\mathrm{Mn}$ to substitute for iron when iron-availability is low would be a simple way to pursue this question. Conversely, the gene may not encode a Mn-transporter but possibly a high-affinity iron transporter. The latter suggestion would be an interesting avenue for future research.

\section{Photosynthesis genes}

The response of photosynthesis genes to iron starvation and recovery is mild given the heavy involvement of iron in photosynthetic electron transfer (Figure 13). We hypothesized that PSI components would be severely affected by iron availability because crystallography shows the association of twelve atoms of iron with the complex (Jordan et al, 2001). Contrary to our hypothesis, only two components of the PSI complex were differentially expressed in each MED4 and MIT9313 (Figure 13, E and F). We observe that the differentially expressed PSI components, psaI, psaL, psaJ, and psaM, are all components with transmembrane $-\alpha$ helices that function to stabilize cofactors of the core antennae system of PSI, which is composed of chlorophylls and carotenoids (Jordan et al, 2001). psaL appears to have a special function in PSI as it is associated with an unidentified metal ion, possibly $\mathrm{Ca}^{2+}$, and may be associated with specific chlorophyll molecules than absorb light at longer wavelengths (red-shift) (Fleming and Grondelle, 1997; Jordan et al, 2001). It will be interesting to determine if the psaL protein and the other ironresponsive components of PSI are replaced during iron stress or if their down-regulation simply represents the initial stages of degradation of the photosystems.

The cytochrome $b_{6} / f$ complex is known to rely on iron to move electrons between PSII and PSI in order to drive ATP synthesis and two of its components were down-regulated in MED4, and one was down-regulated in MIT9313 during iron starvation (Kurisu et al, 2003)

(Figure 13, C and D). In MED4, petA (cytochrome f) and petC (the Rieske iron-sulfur protein) were down-regulated with iron starvation and up-regulated with iron rescue. Both genes clustered 
Figure 13: Response of Prochlorococcus MED4 and MIT9313 photosynthesis genes to iron deprivation and iron rescue. (A,B) MED4 and MIT9313 genes associated with photosystem II. (C,D) MED4 and MIT9313 genes associated with the

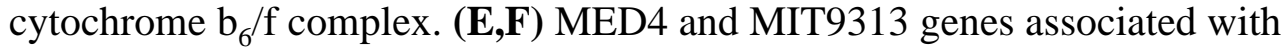
photosystem I. (G,H) MED4 and MIT9313 ATP-synthase genes. Magenta lines represent genes that are differentially expressed in at least one time point or during iron rescue. Turquoise lines represent genes that are marginally differentially expressed in at least one time-point during iron starvation or iron rescue. Gray lines represent genes that are not differentially expressed. Dashed line marks the addition of iron to the experimental treatment to rescue the culture from iron starvation. Horizontal dotted line marks no fold change. 

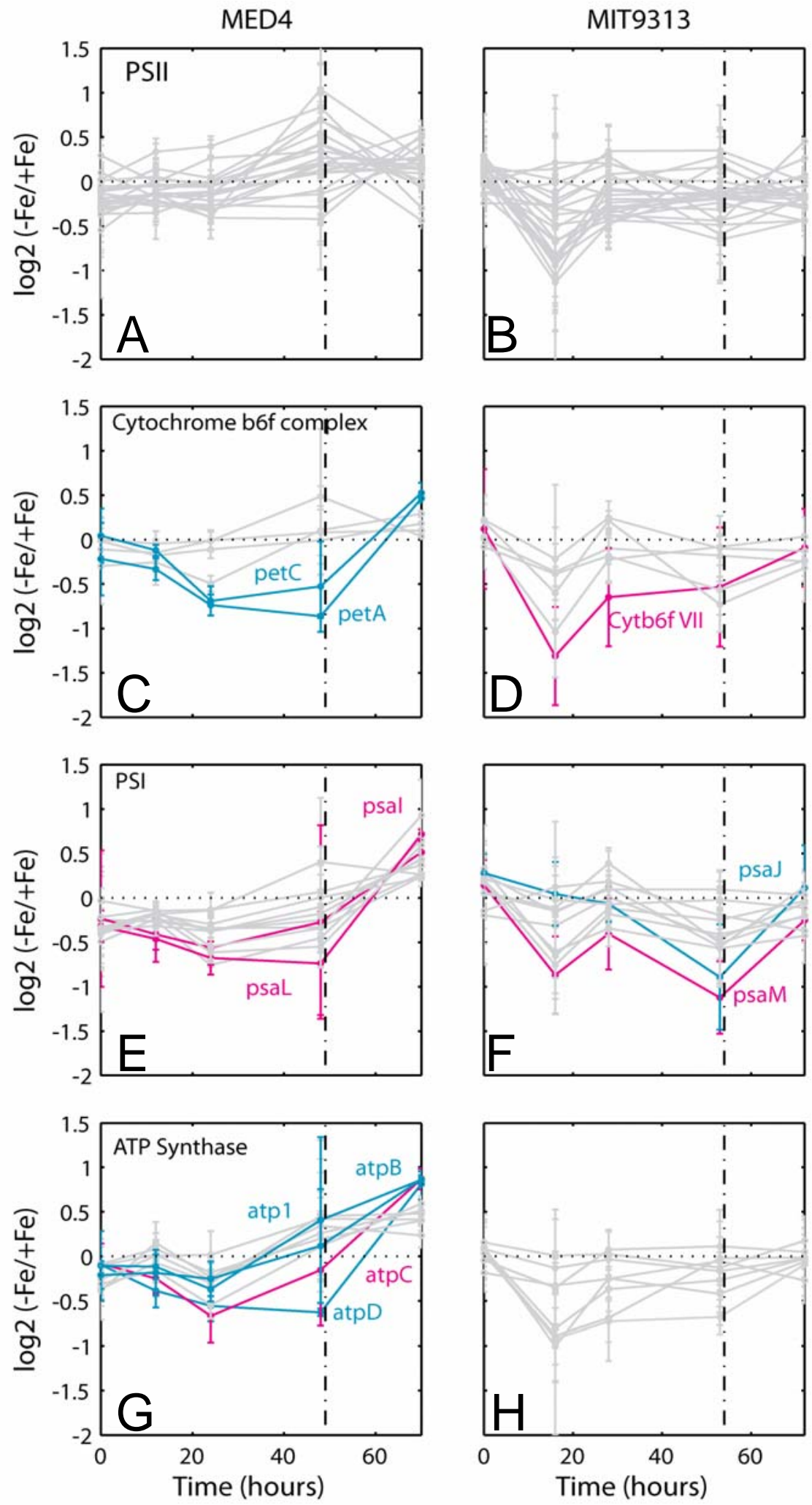
with ferredoxin based on their expression profiles, which makes sense given the association of iron with these two proteins. In MIT9313, P9313_14861 (annotated as a cytochrome b/f complex subunit VII) was significantly down-regulated with iron deprivation and also clustered with ferredoxin among the other MIT9313 genes. It is intriguing that different components of the cytochrome $b_{6} / f$ complex are down-regulated in MED4 and MIT9313 during iron starvation. Our results contrast with an expression study for Synechocystis where cytochrome f and the Rieske Fe-S protein were not differentially expressed in low iron conditions (Singh et al, 2003).

Genes encoding subunits of ATP-synthase were also significantly affected by ironstarvation in MED4 (Figure 13G). In MED4, ATP-synthase subunits atpB, atpC, atpD , and atp1 (a possible ATP synthase subunit 1 - PMM1457) were down-regulated with iron deprivation and clustered together with ferredoxin. In contrast, no ATP-synthase component genes were differentially expressed at a significant level in MIT9313, indicating the success of MIT9313 in protecting its energy-generating system during iron stress in this experiment. Our results for MED4 are consistent with the down-regulation of ATP-synthase components in Synechocystis during iron starvation (Singh et al, 2003).

In contrast to PSI, the cytochromes $b_{6} / f$ complex, and ATP-synthase, no PSII component genes were differentially expressed in either MED4 or MIT9313 (Figure 13, A and B). Our result contrasts with quantitative RT-PCR results published by Bibby et al. 2003 that demonstrate the down-regulation of psbA during iron starvation for MED4, MIT9313, and SS120. In Synechocystis PCC6803, though PSII components psbA2 and psbA3 were unchanged during iron starvation, other PSII components were down regulated (Singh et al, 2003). We believe that it is most likely that our cultures were not iron starved to a severe-enough degree to elicit changes in PSII component gene expression. The down-regulation of PSI in advance of PSII makes sense given the greater iron-requirement of the PSI complex. Bibby et al. 2003 measured the PSI: PSII ratio in iron-replete and iron-starved MIT9313, but found the ratio to remain constant in the two different conditions suggesting that the Prochlorococcus cell is capable of remodeling the PSI complex rather than removing it to conserve iron during situations of iron stress.

\section{Oxidative stress is a consequence of iron starvation in Prochlorococcus}

In response to iron stress, MED4 and MIT9313 both expressed genes that are typically associated with oxidative stress. Most prominently, both Prochlorococcus ecotypes up-regulated several high-light inducible protein (hlips) genes during iron starvation that were down-regulated 

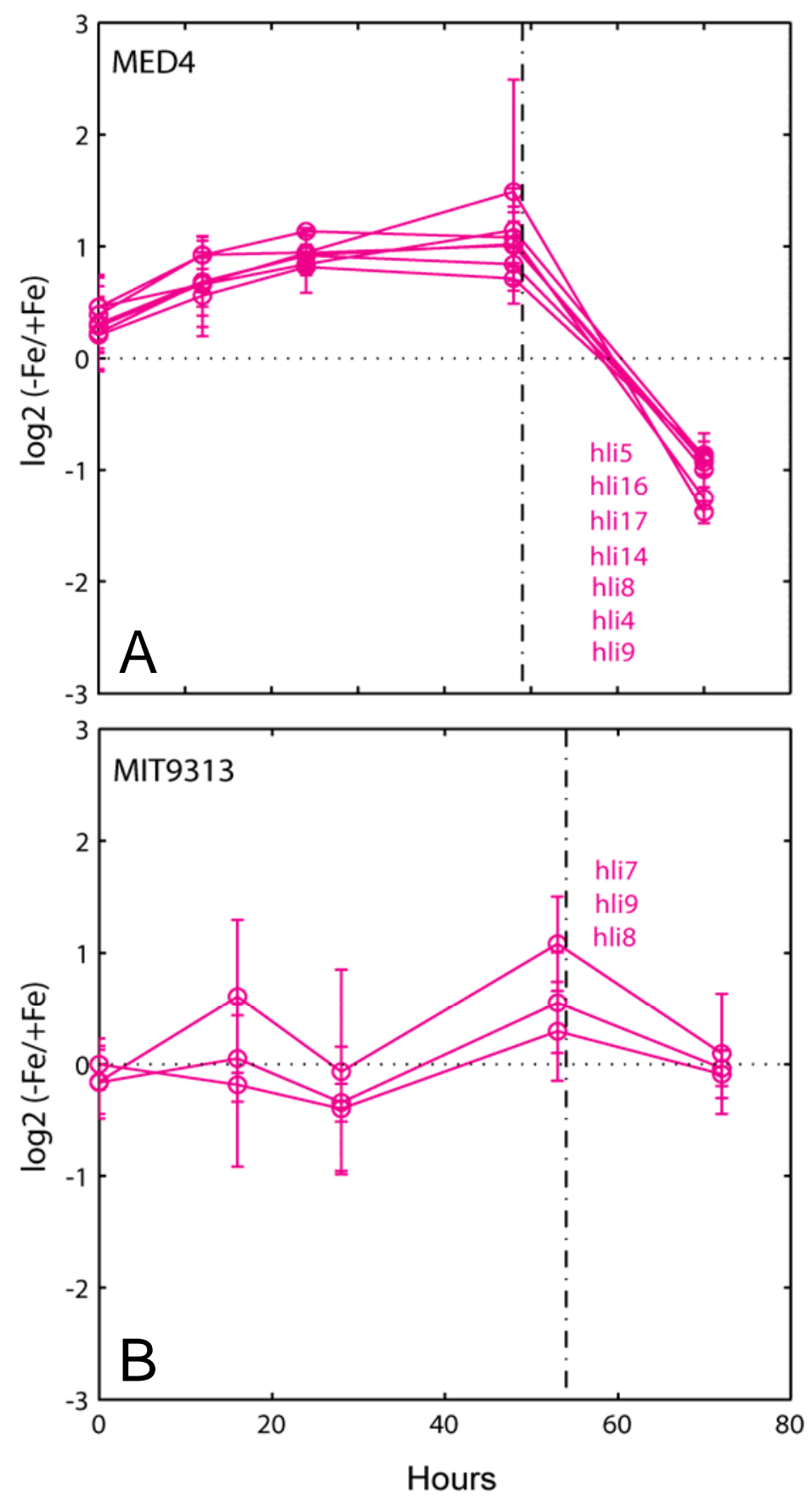

Figure 14: Response of Prochlorococcus MED4 and MIT9313 high-light inducible protein genes (hlips) to iron deprivation and iron rescue. (A) MED4. (B) MIT9313. Dashed line marks the addition of iron to the experimental treatment to rescue the culture from iron starvation. Horizontal dotted line marks no fold change. 
following iron rescue. In MED4, hli04, hli05, hli08, hli09, hli14, hli16, and hli17 responded to iron availability and clustered together with flavodoxin (Figure 14). Several other hlips were marginally differentially expressed. In MIT9313, hli07, hli08, and hli09 responded to iron availability though the pattern of differential expression over the time-course varied from MED4's hlips (Figure 14). The MIT9313 hlips were only slightly up-regulated over the timecourse of the experiment but did not respond significantly to iron addition to the experimental treatment.

A bacterioferritin comigratory protein (bcp) was also strongly significantly up-regulated in MED4 and is homologous to the bcp in E.coli where it is characterized as a thiol peroxidase and is induced during oxidative stress (Jeong et al, 2000). Both MED4 and MIT9313 genomes contain two copies of the bacterioferritin comigratory proteins (not to be confused with bacterioferritin, which may have a role in iron storage but is not present in the MED4 and MIT9313 genomes) but only one, PMM0345 in MED4, is differentially expressed in response to iron deprivation and is one of the most highly differentially-expressed genes in the MED4 genome in response to iron deprivation. This result suggests that MED4 has multiple methods of dealing with oxidative stress induced by low iron availability. Possibly, at this level of iron deprivation, MIT9313 experiences less oxidative stress than MED4 or is capable of mitigating oxidative stress more effectively.

\section{Regulation of iron metabolism in Prochlorococcus}

Small RNAs (sRNAs) dominate the regulatory response to iron availability suggesting a major role for sRNAs in the regulation of Prochlorococcus iron metabolism (Figure 15). Of the 17 ncRNAs recently identified and 7 previously known ncRNAs in MED4, eight responded significantly to iron-stress or recovery from iron-stress. Of the 24 asRNAs that were also recently identified in MED4, two responded significantly to iron availability (Figure 15). Also, in MED4, the putative principal RNA polymerase sigma factor (sigA - PMM0496) is down-regulated with iron addition indicating that multiple systems are active in regulating the iron response in MED4.

The activity of sRNAs in the Prochlorococcus iron stress response is not surprising given the recent discovery of the involvement of sRNAs in regulating the iron metabolism of E.coli and regulating isiA expression in Synechocystis (Duhring et al, 2006). RhyB is a small RNA that represses genes that encode iron-requiring proteins or iron-storage proteins during conditions of low iron availability when its repressor, fur (ferric uptake regulator) is not bound to iron and 

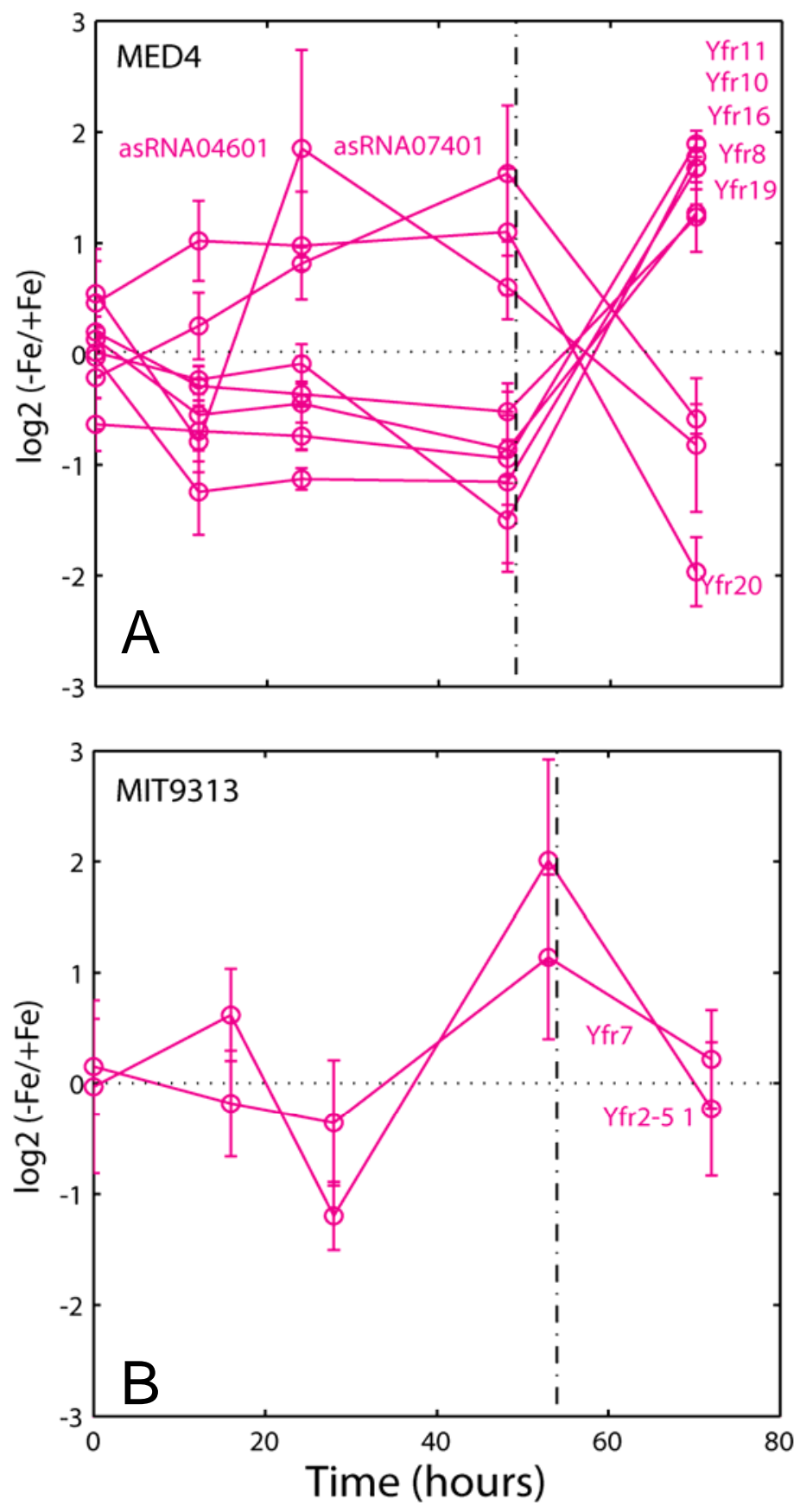

Figure 15: Response of Prochlorococcus MED4 and MIT9313 small non-coding RNAs to iron deprivation and iron rescue. (A) MED4. (B) MIT9313. Dashed line marks the addition of iron to the experimental treatment to rescue the culture from iron starvation. Horizontal dotted line marks no fold change. 
cannot bind to its target DNA (Masse et al, 2002). Therefore, when iron is abundant, rhyB cannot repress iron-requiring proteins. Because none of the small RNAs in Prochlorococcus have homologs in other organisms (Steglich et al, 2008), we do not expect to find a rhyB homolog in Prochlorococcus but we do hypothesize that a small RNA of similar function exists in Prochlorococcus. Therefore, we hypothesize that the two iron-starvation induced anti-sense RNAs, asRNA_04601 and asRNA_07401, repress iron-requiring proteins and are repressed by fur when iron is present. The target of asRNA_04601 is a conserved hypothetical gene whose target (PMED4_04601) is not differentially expressed. The target of asRNA_07401, PMED4 _ 07401, is a possible Zn-finger protein. Though not an anti-sense RNA, the behavior of Yfr20 suggests a similar regulatory function as RhyB, as well. Notably, Yfr20 was shown to be transcribed as a dicistronic element with PMED4_15791 (conserved hypothetical) which was constitutively expressed under light stress though Yfr20 was up-regulated suggesting that the light-dependent expression of Y fr20 is controlled by its own promoter (Steglich et al, 2008). In contrast, during iron limitation Yfr20 clusters with PMED4_15791, which is marginally differentially expressed (yet, with a strong q-value of $<0.0001$ ), demonstrating that its mode of expression during iron stress is different than the light-dependent expression (Steglich et al, 2008). Furthermore, we hypothesize possible roles for the iron-induced ncRNAs (Yfr 8, 10, 11, 16 , and 19) in repressing high-affinity iron-acquisition genes or proteins that replace ironcontaining proteins during low iron conditions, like flavodoxin.

Of the three known small regulatory RNAs in MIT9313, Yfr7 and Yfr2-5_1 were initially down-regulated with the removal of iron from the media then up-regulated during later stages of iron limitation but belong to separate clusters of expression profiles (Figure 15) (Table 3). It is likely that the paucity of small RNAs in MIT9313 reflects the fact that those in Prochlorococcus have largely been identified using MED4 and not because of a smaller role for small RNAs in regulation in MIT9313 than MED4. It will be interesting to compare small RNAs in MED4 and MIT9313 once more are identified in MIT9313.

Unexpectedly, Prochlorococcus homologs to the ferric uptake regulator (fur) were not differentially expressed, even marginally, in either MED4 (two copies) or MIT9313 (three copies) during iron starvation or rescue. Further clues to the role of fur in regulating the iron metabolism of Prochlorococcus can be gained from the diel light-availability experiment. Under diel conditions, the expression of one copy of fur in MED4 follows a cyclical pattern with peak expression occurring at sunset (Figure 8) (Zinser et al, 2009). The expression of this copy of fur 

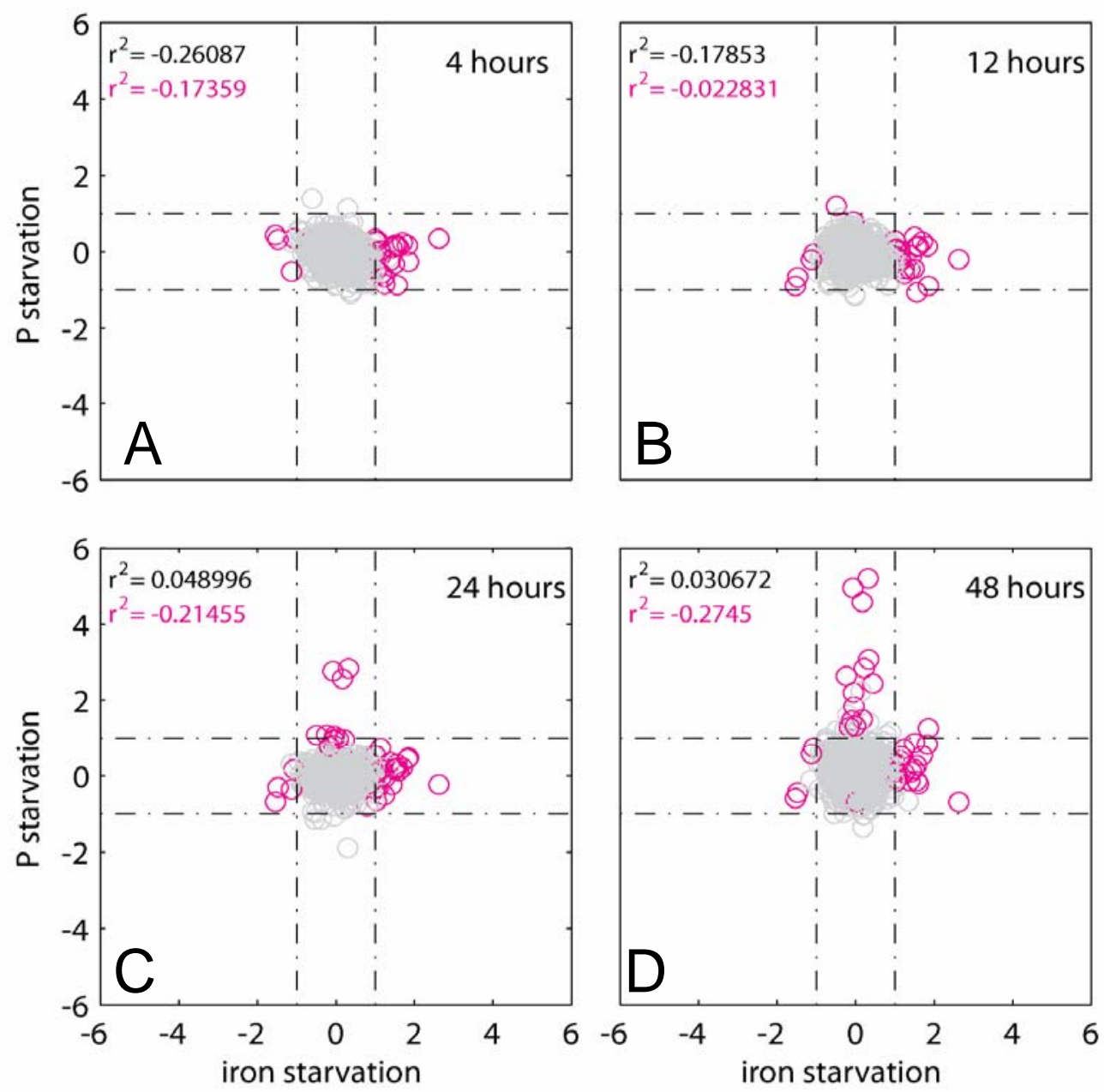

Figure 16: Comparison of the global expression response in Prochlorococcus MED4 to iron starvation at 24 hours (x-axis) and all time-points of phosphorous starvation (yaxis). (A-D) Response to phosphate starvation between 4 and 48 hours after the initiation of starvation compared to iron starvation. All values represent the log2 (fold change) of experimental compared to control conditions. Data for global expression in response to phosphate availability is from Martiny and Coleman et al. 2006. Magenta circles represent genes that are differentially expressed in the iron experiment or phosphorous experiment. Gray circles represent genes that were not differentially expressed in both experiments. Correlation coefficients $\left(\mathrm{r}^{2}\right)$ indicate how well the fold change for each probeset compares between the two experiments with magenta representing the correlation of differentially-expressed genes and gray representing the correlation between non differentially-expressed genes. Dashed lines indicate the location of fold change threshold (1 or -1$)$ for differentially-expressed genes. 

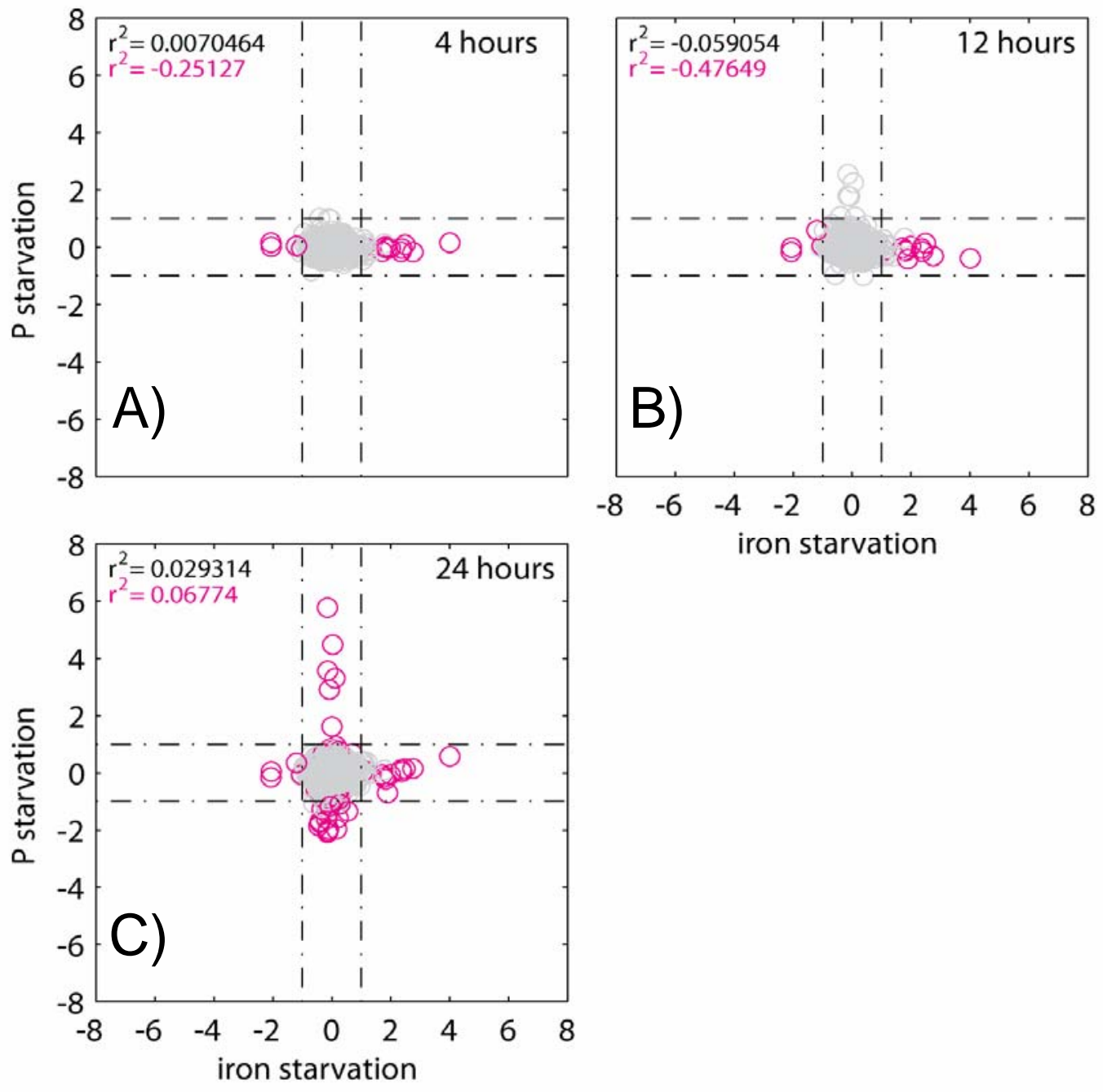

Figure 17: Comparison of the global expression response in Prochlorococcus MIT9313 to iron starvation at 24 hours (x-axis) and all time-points of phosphorous starvation (y-axis). (A-C) Response to phosphate starvation between 4 and 24 hours after the initiation of starvation compared to iron starvation. All values represent the log2 (fold change) of experimental compared to control conditions. Data for global expression in response to phosphate availability is from Martiny and Coleman et al. 2006. Magenta circles represent genes that are differentially expressed in the iron experiment or phosphorous experiment. Gray circles represent genes that were not differentially expressed in both experiments. Correlation coefficients $\left(\mathrm{r}^{2}\right)$ indicate how well the fold change for each probeset compares between the two experiments with magenta representing the correlation of differentially-expressed genes and gray representing the correlation between non differentially-expressed genes. Dashed lines indicate the location of fold change threshold (1 or -1) for differentially-expressed genes. 
in the diel experiment gives us confidence that there is a role for fur in Prochlorococcus. Interestingly, the other copy of fur in MED4 is aperiodic in expression over the diel cycle, as is ferredoxin (Appendix B, Figure 19). By examining the diel expression of fur, the known irontransporters afuA/idiA, futB, and futC, as well as flavodoxin (isiB), we question whether the fluctuation in the expression of these genes reflects changes in intracellular iron concentration, changes in media iron concentrations (via photoreduction of Fe(III) from EDTA-Fe complexes), or both (Sunda and Huntsman, 2003). The role of fur in binding iron in the cell during high-iron concentrations and the expression of $i s i B$ in response to iron starvation conditions suggest the highest iron concentrations in the cell near sunset, or the time of cell division (Figure 8).

It has been hypothesized that one advantage of using sRNAs in regulation is to enable quick and efficient (i.e. no protein synthesis is required) responses to changing conditions. It will be very interesting to describe the interaction between fur and the more dynamic sRNAs that play a role in Prochlorococcus iron metabolism. We can imagine a scenario where the role of fur in regulating the iron metabolism has been usurped by the more dynamic small RNAs, where fur and the sRNAs control different aspects of the cell's response to changing iron availability, or a system that is dependent on the interaction of fur and the small RNAs to regulate the iron response as in the fur-RhyB system of E.coli (Masse et al, 2002; Masse et al, 2005).

\section{Comparison of iron stress response to that of other stress conditions}

In order to understand how iron metabolism influences other aspects of cellular metabolism we compared the iron-stress whole-genome response to the nitrogen and phosphorus starvation responses, response to shifts in light availability, and response to phage infection. There is almost no similarity in the whole-genome expression of MED4 and MIT9313 to iron starvation and phosphorus starvation in any time-point (Figures 16 and 17). Likewise, the wholegenome response to phage infection does not closely resemble iron stress in MED4 (Figure 18). There is some similarity in the stress response of MED4 to iron and late-stage nitrogen starvation though not in MIT9313 (Figures 19 and 20). Finally, the response of MED4 to changes in light quality and quantity displays strong similarities to the iron stress response (Figures 21).

Both MED4 and MIT9313 are capable of growing on sole nitrogen sources of ammonium and urea and have the appropriate genes corresponding to uptake and utilization (Moore et al, 2002; Rocap et al, 2003). Yet, only MIT9313 is capable of assimilating nitrite and only MED4 is capable of utilizing cyanate (Garcia-Fernandez et al, 2004; Kettler et al, 2007; Moore et al, 2002; 
Figure 18: Comparison of the global expression response in Prochlorococcus MED4 to iron starvation at 24 hours (x-axis) and all time-points of phage infection (y-axis). (A-H) Response to phage infection at 1-8 hours after infection compared to iron starvation. All values represent the log2 (fold change) of experimental compared to control conditions. Data for global expression in response to phage infection is from Lindell et al. 2007. Magenta circles represent genes that are differentially expressed in the iron experiment or phage infection experiment. Gray circles represent genes that were not differentially expressed in both experiments. Correlation coefficients $\left(\mathrm{r}^{2}\right)$ indicate how well the fold change for each probeset compares between the two experiments with magenta representing the correlation of differentially-expressed genes and gray representing the correlation between non differentially-expressed genes. Dashed lines indicate the location of fold change threshold (1 or -1$)$ for differentially-expressed genes. 

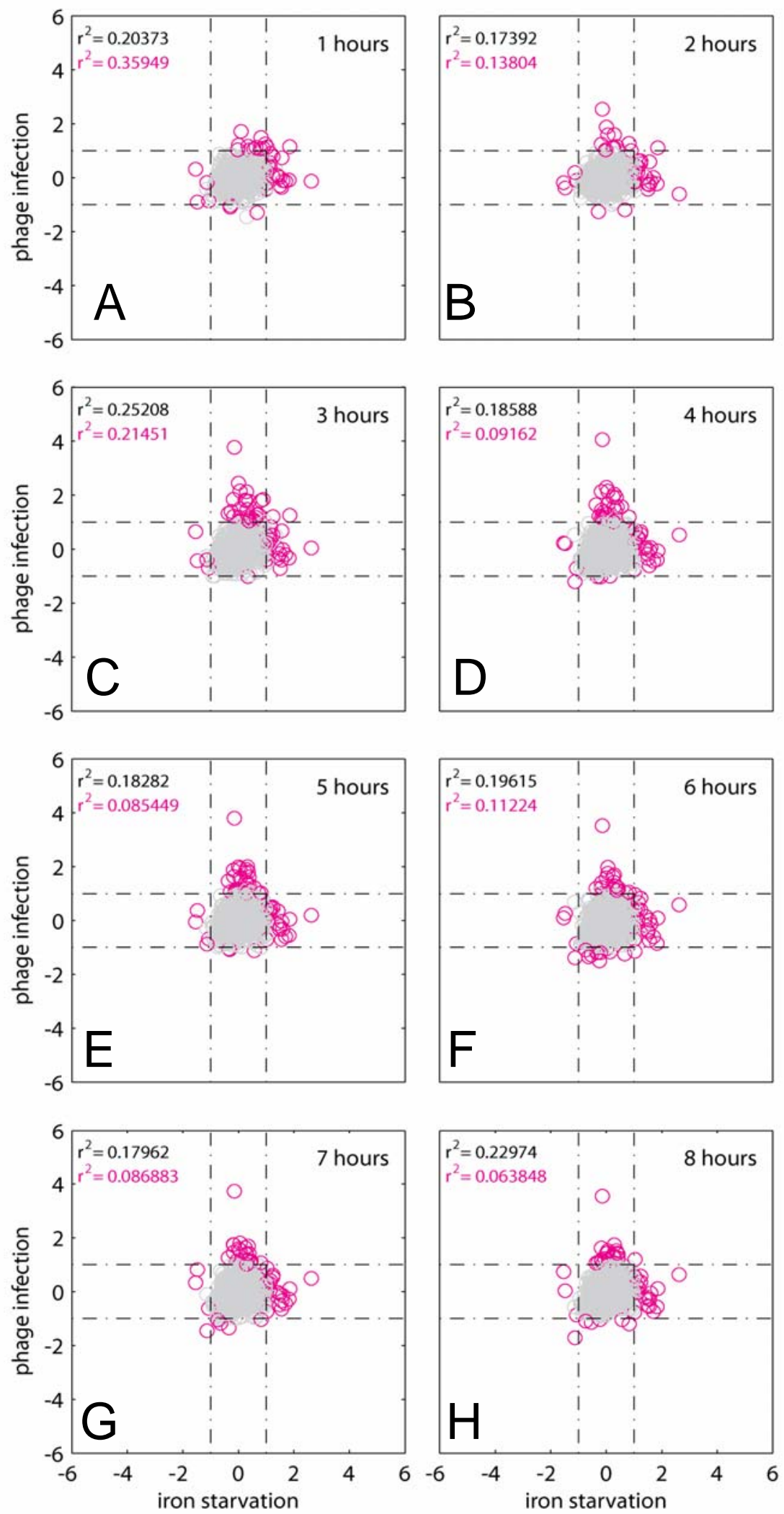
Figure 19: Comparison of the global expression response in Prochlorococcus MED4 to iron starvation at 24 hours (x-axis) and all time-points of nitrogen starvation (yaxis). (A-E) Response to ammonium starvation between 3 and 48 hours after the initiation of starvation compared to iron starvation. (F,G) Steady-state growth on urea or cyanate as compared to a control of growth on ammonium compared to iron starvation. All values represent the log2 (fold change) of experimental compared to control conditions. Data for global expression in response to nitrogen availability is from Tolonen et al. 2006. Magenta circles represent genes that are differentially expressed in the iron experiment or nitrogen experiment. Gray circles represent genes that were not differentially expressed in both experiments. Correlation coefficients $\left(\mathrm{r}^{2}\right)$ indicate how well the fold change for each probeset compares between the two experiments with magenta representing the correlation of differentially-expressed genes and gray representing the correlation between non differentially-expressed genes. Dashed lines indicate the location of fold change threshold ( 1 or -1 , for iron) (1.5 or -1.5 , for $\mathrm{N}$ ) for differentially-expressed genes. 

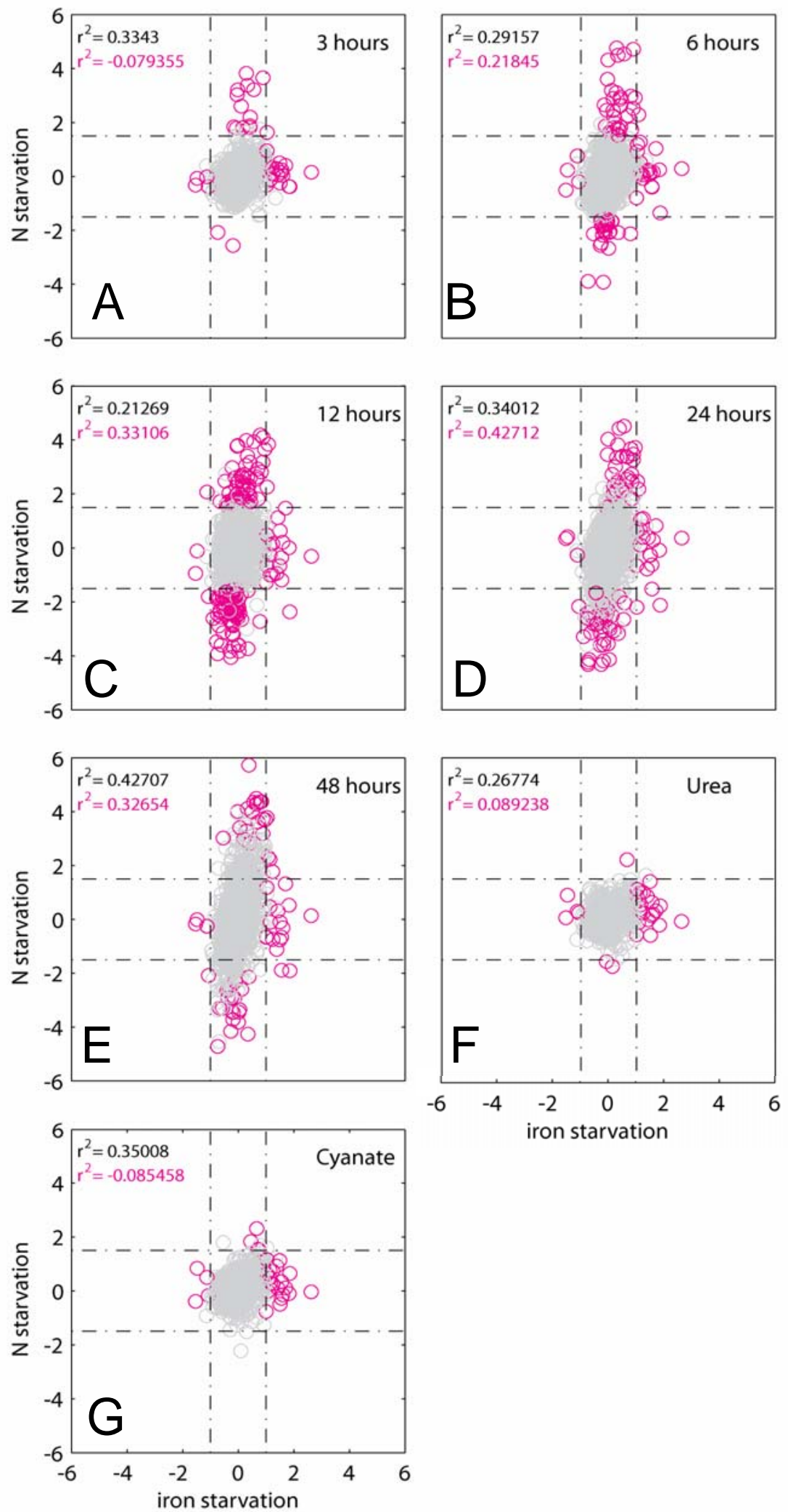
Figure 20: Comparison of the global expression response in Prochlorococcus MIT9313 to iron starvation at 28 hours (x-axis) and all time-points of nitrogen starvation (y-axis). (A-E) Response to ammonium starvation between 3 and 48 hours after the initiation of starvation compared to iron starvation. $(\mathbf{F}, \mathbf{G})$ Steadystate growth on urea or nitrite as compared to a control of growth on ammonium compared to iron starvation. All values represent the log2 (fold change) of experimental compared to control conditions. Data for global expression in response to nitrogen availability is from Tolonen et al. 2006. Magenta circles represent genes that are differentially expressed in the iron experiment or nitrogen experiment. Gray circles represent genes that were not differentially expressed in both experiments. Correlation coefficients $\left(\mathrm{r}^{2}\right)$ indicate how well the fold change for each probeset compares between the two experiments with magenta representing the correlation of differentially-expressed genes and gray representing the correlation between non differentially-expressed genes. Dashed lines indicate the location of fold change threshold ( 1 or -1 , for iron) ( 2 or -2 , for $\mathrm{N}$ ) for differentially-expressed genes. 

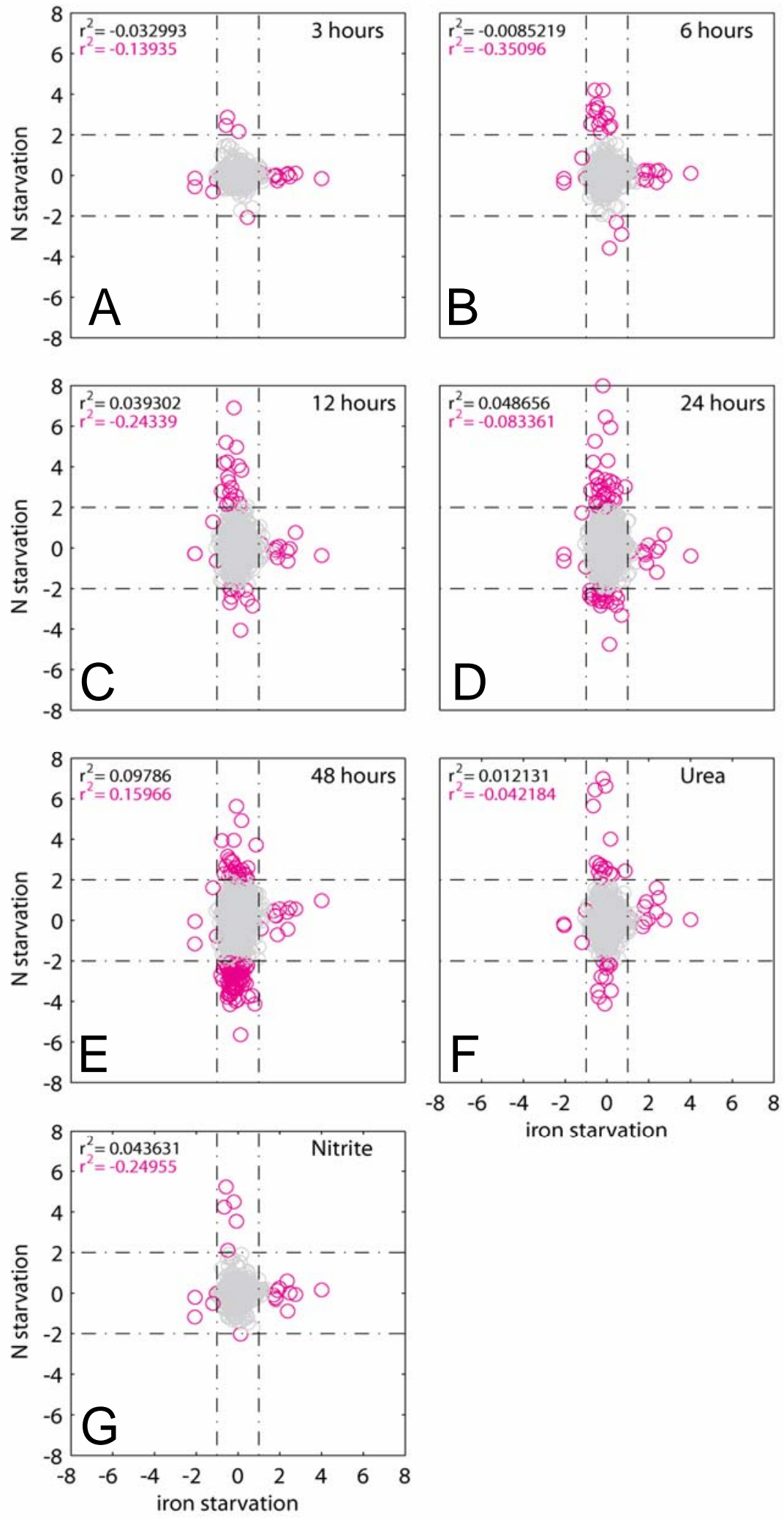
Figure 21: Comparison of the global expression response in Prochlorococcus MED4 to iron starvation at 24 hours (x-axis) and shifts in light availability (y-axis). (A-E) Response to shift between the dark and blue, green, red, high light, or white light compared to iron starvation. (F) Response to DCMU addition of a culture acclimated to white light compared to iron starvation. All values represent the log2 (fold change) of experimental compared to control conditions. Data for global expression in response to light quality and quantity shifts is from Steglich et al. 2006. Magenta circles represent genes that are differentially expressed in the iron experiment or light experiment. Gray circles represent genes that were not differentially expressed in both experiments. Correlation coefficients $\left(\mathrm{r}^{2}\right)$ indicate how well the fold change for each probeset compares between the two experiments with magenta representing the correlation of differentially-expressed genes and gray representing the correlation between non differentially-expressed genes. Dashed lines indicate the location of fold change threshold (1 or -1 ) for differentially-expressed genes. 

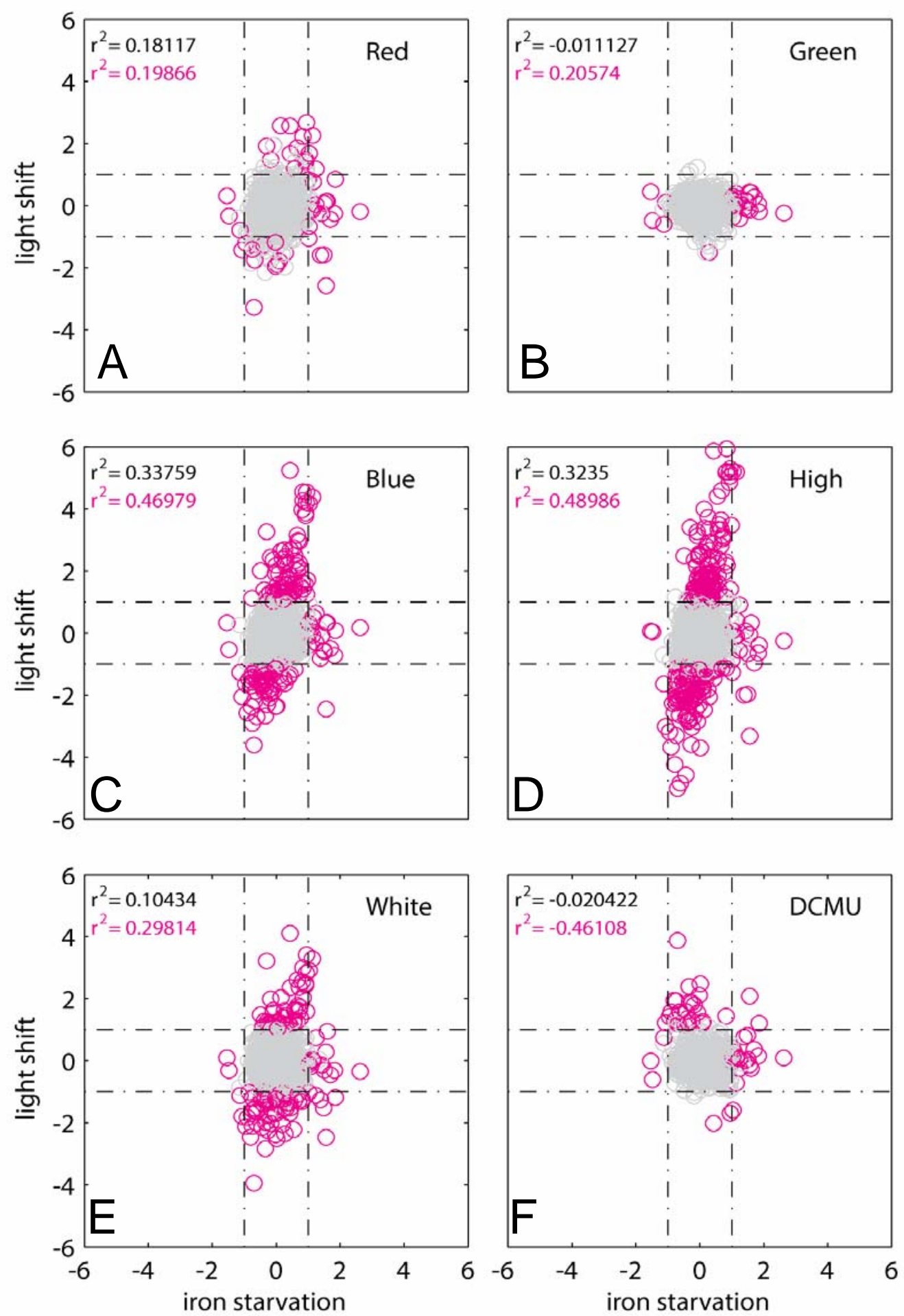
Rocap et al, 2003). Thus, we hypothesized that a difference in nitrogen physiology could be responsible for the difference in iron physiology of MED4 and MIT9313 if one mechanism of assimilating nitrogen involves more iron than another (Chapter 2). We found that at early timepoints of nitrogen starvation and growth on alternative nitrogen sources, the iron-stress response was well-correlated to the nitrogen-stress response in MED4 or MIT9313 (Figure 19 and 20). Thus, we expect that iron does not play a strong role in nitrogen metabolism, compared to photosynthesis (see below), on any of the tested nitrogen sources like it does during nitrogen fixation (Berman-Frank et al, 2001; Hernandez et al, 2008). We hypothesize that the difference in nitrogen physiologies of MED4 and MIT9313 is not related to the tolerance of MIT9313 for low iron concentrations in comparison to MED4 and conclude that the use of alternative nitrogen sources, such as urea, cyanate, and nitrite does not depend on iron availability.

At later time-points of nitrogen starvation in MED4 (12-24 hours), we observe an increasing similarity with the iron stress response that is not seen at the later points of nitrogen starvation in MIT9313 (Figure 19 and 20). Relative bulk fluorescence decreased in both MED4 and MIT9313 nitrogen starvation experiments starting at 12 hours after the initiation of starvation and though cell numbers are not available, we assume this point marked the onset of severe stress in the nitrogen-starved cells and possibly a decline in the cell-division rate (Tolonen et al, 2006). We hypothesize that in the later stages of nitrogen starvation, and during iron starvation, MED4 cultures commonly experience oxidative stress.

Shifts from darkness to blue light, white light, high intensity of white-light, and addition of DCMU to a white-light acclimated culture were most similar to iron starvation out of the shifts in light quantity and quality that were tested (Figure 21). A few genes were differentially expressed in iron starvation and during exposure to red light. And, as previously published, exposure to green light invokes only minimal changes in MED4 gene expression and no greenlight responsive genes were differentially expressed with iron rescue (Steglich et al, 2006).

High light inducible genes were commonly up-regulated during exposure to red, blue, white, and high light and were induced during iron starvation. The response of hli genes in both stress conditions suggests that both light and iron stress are linked to oxidative stress as hlips are thought to play a role in protecting cells from oxidative stress (see above). Interestingly, MED4 genes that were up-regulated during iron stress were down-regulated upon exposure to DCMU (Figure 21F). DCMU is known to cause oxidation of the plastoquinone pool so suggests that iron stress causes reduction of the plastoquinone pool. The reduction of the plastoquinone pool during 
iron stress suggests that during conditions of iron starvation, electrons are capable of flowing to the plastoquinone pool from PSII but transfer of the electrons down-stream to the cytochrome $b_{6} / f$ complex is inhibited or slowed. The slow-down of photosynthetic electron transfer could be due to the down-regulation of some components of the cytochrome $b_{6} / f$ complex during iron stress as we see in Figure 13.

\section{Genes of unknown function}

Genes of unknown function make up $\sim 60 \%$ and $\sim 65 \%$ of the iron-responsive genes in MED4 and MIT9313, respectively. Remarkably, none of these genes differentially expressed in both strains. A few of the iron-responsive genes of unknown function are of particular interest either because of their proximity to genes known to be related to iron or because of the magnitude of their expression. PMM1170 in MED4 is strongly induced during iron starvation and is present in the MIT9313 genome (PMT0821), though not differentially expressed. It is also present in all other sequenced Prochlorococcus genomes and several Synechococcus genomes. In MED4, PMM1170 is directly down-stream of the iron-stress induced protein (isiB, flavodoxin) that is classically up-regulated during iron-stress. PMM1170 and isiB cluster together in the iron experiments and in the diel experiment (Zinser et al, 2009) (Appendix B, Figure 19) suggesting that PMM1170 and isiB are dicistronic in MED4. Yet, they could also be regulated in the same way if they possess similar iron-regulatory sequences. In the other high-light adapted genomes, orthologs of PMM1170 are separated from isiB by one gene, $p c b D$, a chlorophyll-binding protein (microbesonline.org). In the low-light adapted Prochlorococcus genomes and in Synechococcus, orthologs to PMM1170 and isiB are not in close proximity. Like isiB, PMM1170 is not differentially expressed during other conditions of stress (N, P, light, or phage) suggesting a specific role in the iron metabolism of MED4. Elaborating on the function of these many uncharacterized iron-responsive genes will be an important aspect of fully characterizing the iron metabolism of Prochlorococcus and the ecology of Prochlorococcus.

\section{Conclusion and Future directions}

In this study, we measured the whole-genome response of Prochlorococcus MED4 and MIT9313 to changes in iron availability. We sought both to understand the molecular underpinnings of MIT9313's capability of surviving at lower iron concentrations than MED4 (Chapter 2), and to elucidate the iron-uptake, use, storage, and regulatory mechanisms that define 
Prochlorococcus and contribute to the diversity of its ecotypes. In general, we found that the Prochlorococcus iron metabolism is encoded in both the core genome and in the non-core genome. Thus, gradients in iron availability in the natural environment have also shaped the ecology and diversity of this group of organisms.

As is often the case with whole-genome expression studies, we have raised many more questions about the iron metabolism of Prochlorococcus than we have answered and there are several avenues open for future work. First, it will be interesting to further characterize the ironresponsive conserved hypothetical and hypothetical genes that we have identified in this study. Still, we cannot recognize a complete iron-acquisition system in Prochlorococcus and identifying transporters among the genes of unknown function would be a significant finding. It will be very interesting to see whether the sequences of any unidentified components of iron transporters in Prochlorococcus are related to other known iron-transporters or represent novel mechanisms of iron acquisition in the open-ocean. The genes of unknown function that are down-regulated in response to iron stress would be good candidates for genes encoding proteins that use iron, such as ferredoxin. Learning the function of these genes will help us better describe the cellular processes in Prochlorococcus that rely on iron availability and contribute to its iron quota. Theoretically, the construction of knock-out mutants in Prochlorococcus is the simplest way to test the function of the uncharacterized iron-responsive genes. Yet, no reliable genetic system is available for Prochlorococcus at this time. Many of these genes have homologs in other, more easily genetically manipulated organisms in which knock-out mutants for the most interesting of the iron-responsive genes could be created and screened.

We are also motivated to examine the effects of more extreme iron stress on wholegenome expression in Prochlorococcus. The current experiment dealt with cultures that were iron stressed but did not show dramatic differences in growth rate between the control and treatment cultures. Does the whole-genome expression response to iron-limitation differ from the response to iron-stress? This question may be answered by measuring gene expression in cells during steady-state iron limitation after several transfers in low iron media. We have attempted to measure gene expression during steady-state iron limitation but poor replication prevents robust interpretation of the expression results.

Finally, the complexities and unknowns of post-transcriptional regulation and translation limit the power of transcriptional analyses, such as microarray experiments. We are very interested in understanding how the Prochlorococcus proteome responds to iron stress as well as 
identifying the differences between the transcriptional and translational response of Prochlorococcus to iron availability. We are especially intrigued to see if some of the non-iron responsive but predicted iron-related genes, like the iron transporter component futB and regulator fur, respond to iron stress at the translational level. Proteomic analysis is also appealing as we may be able to learn about the location of uncharacterized iron-responsive genes within the Prochlorococcus cell.

\section{Acknowledgements}

We would like to acknowledge Debbie Lindell for developing the custom M4-9313 Affymetrix array, experimental design, and RNA extraction methods. Thanks to Sarah Annis and Trent Rector at the Harvard Biopolymers Facility for testing the quality of RNA samples and processing the microarray samples. We also thank Libusha Kelly for helpful discussions on array analysis and interpretation of expression results. 


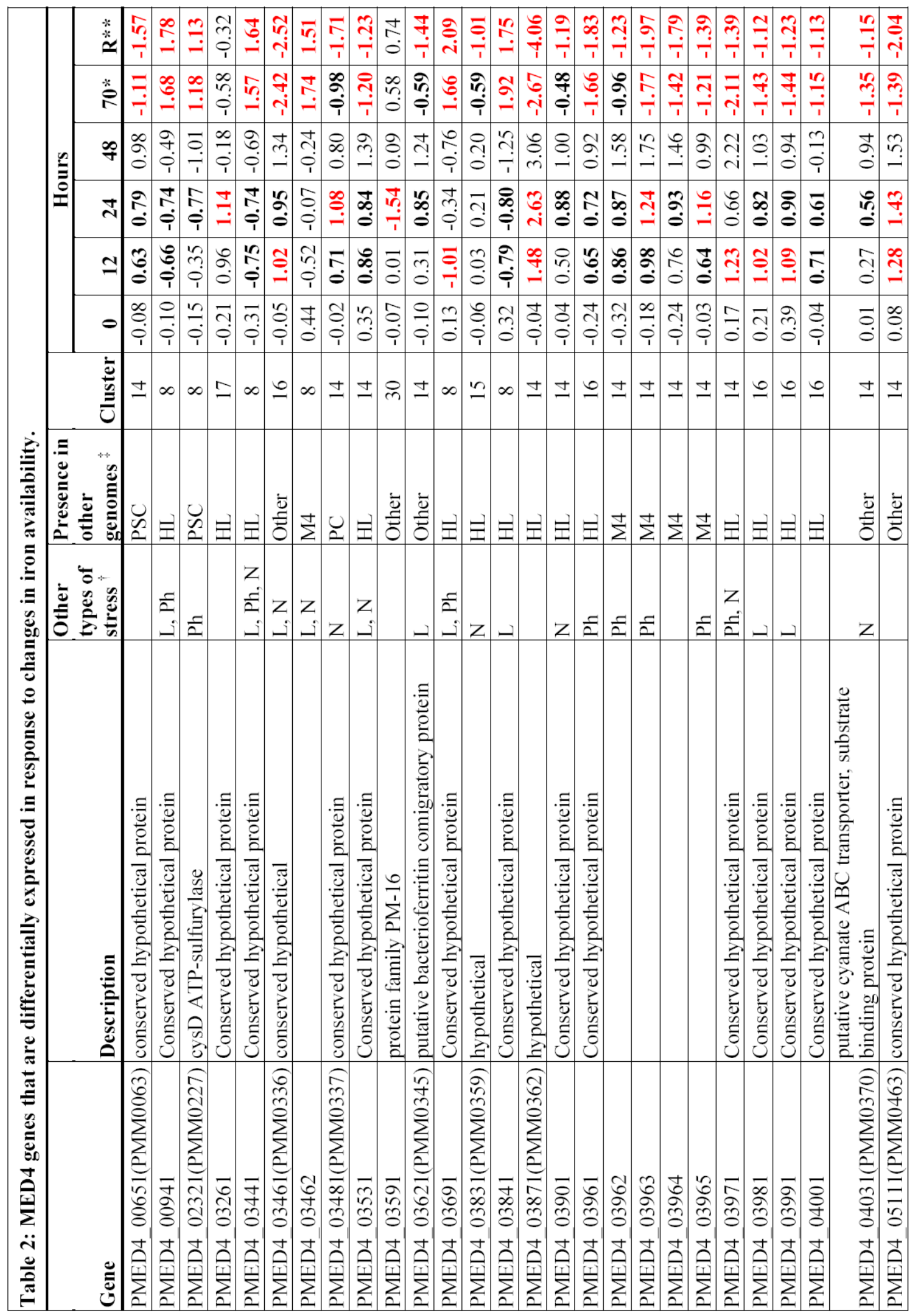




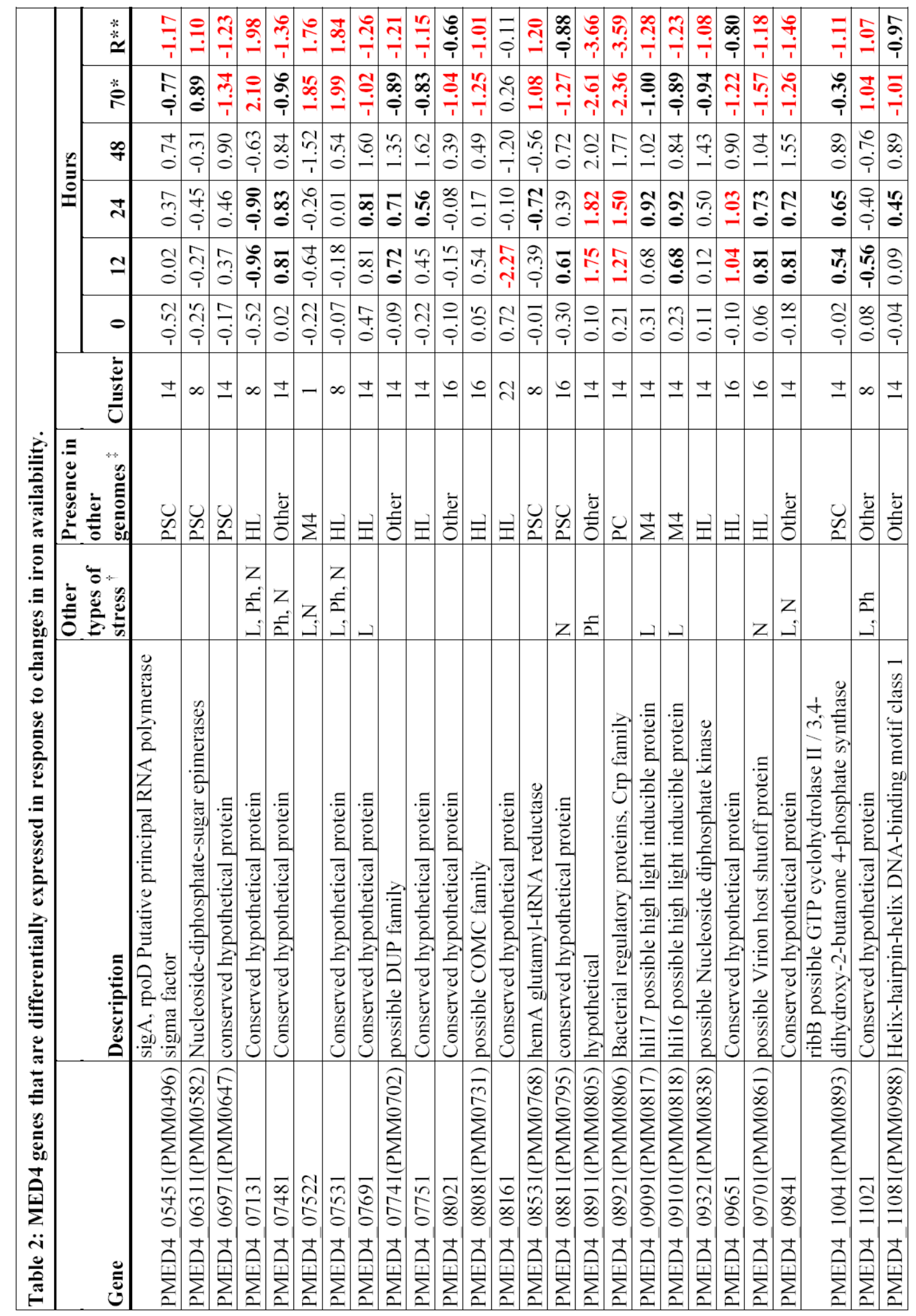




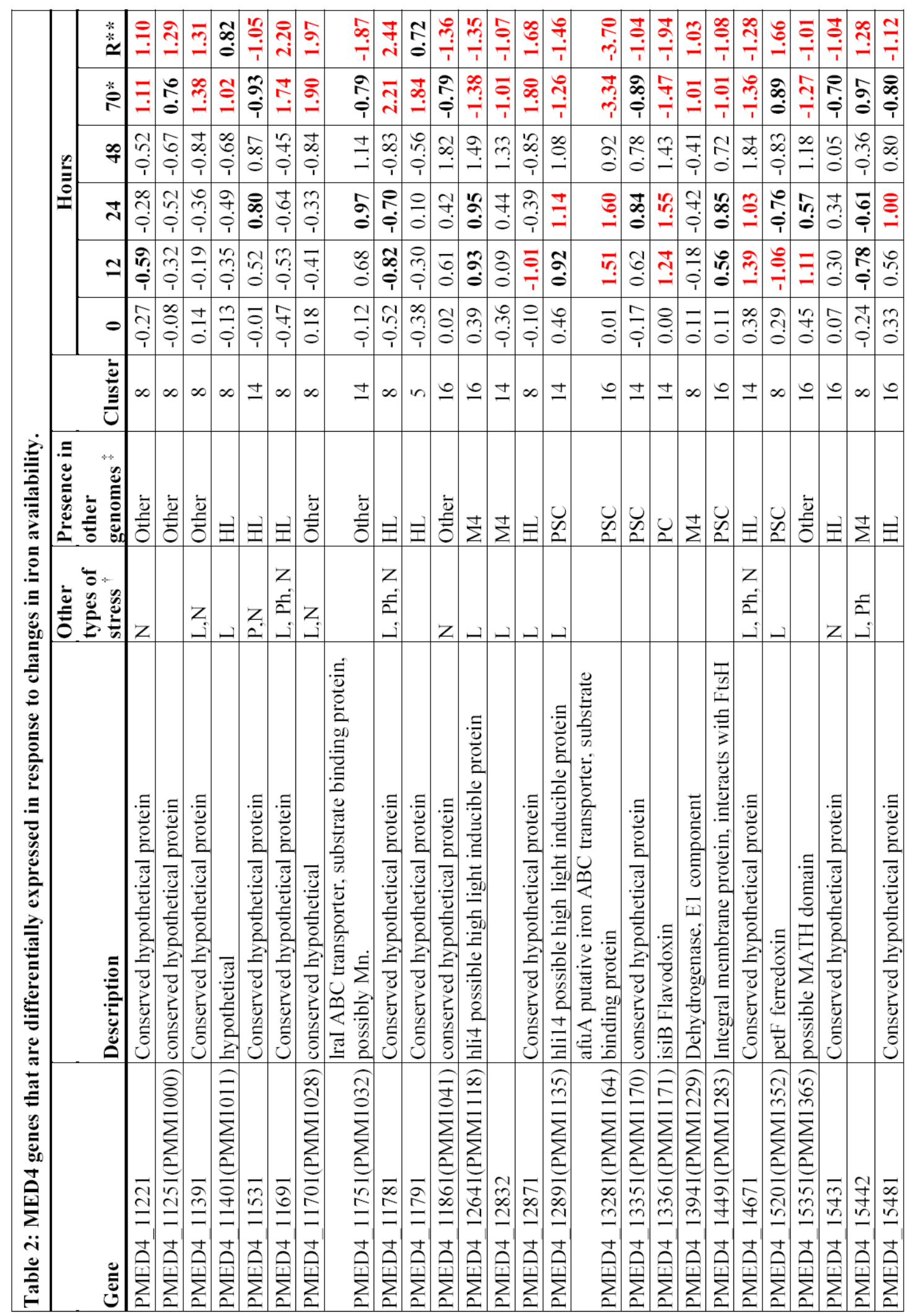




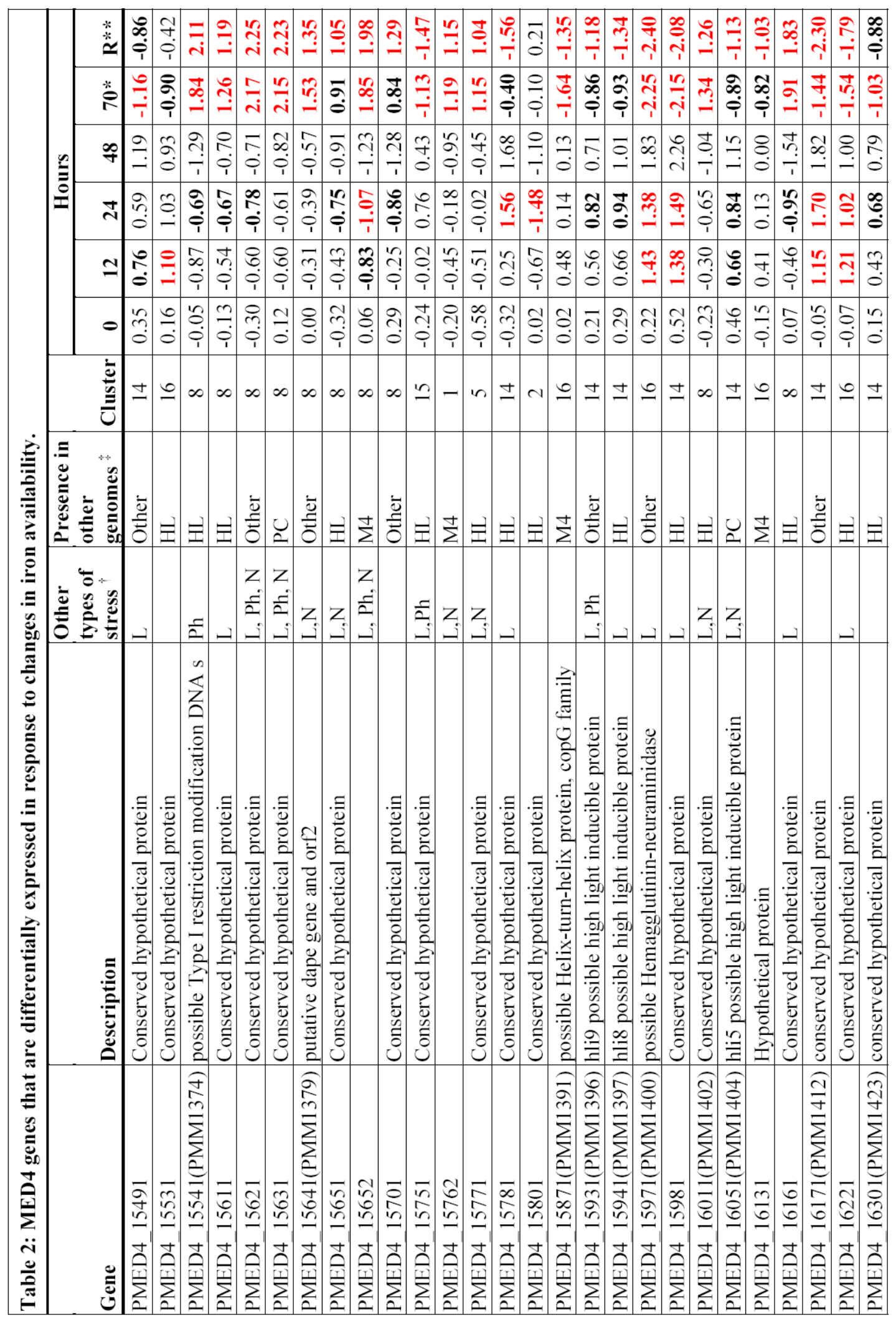




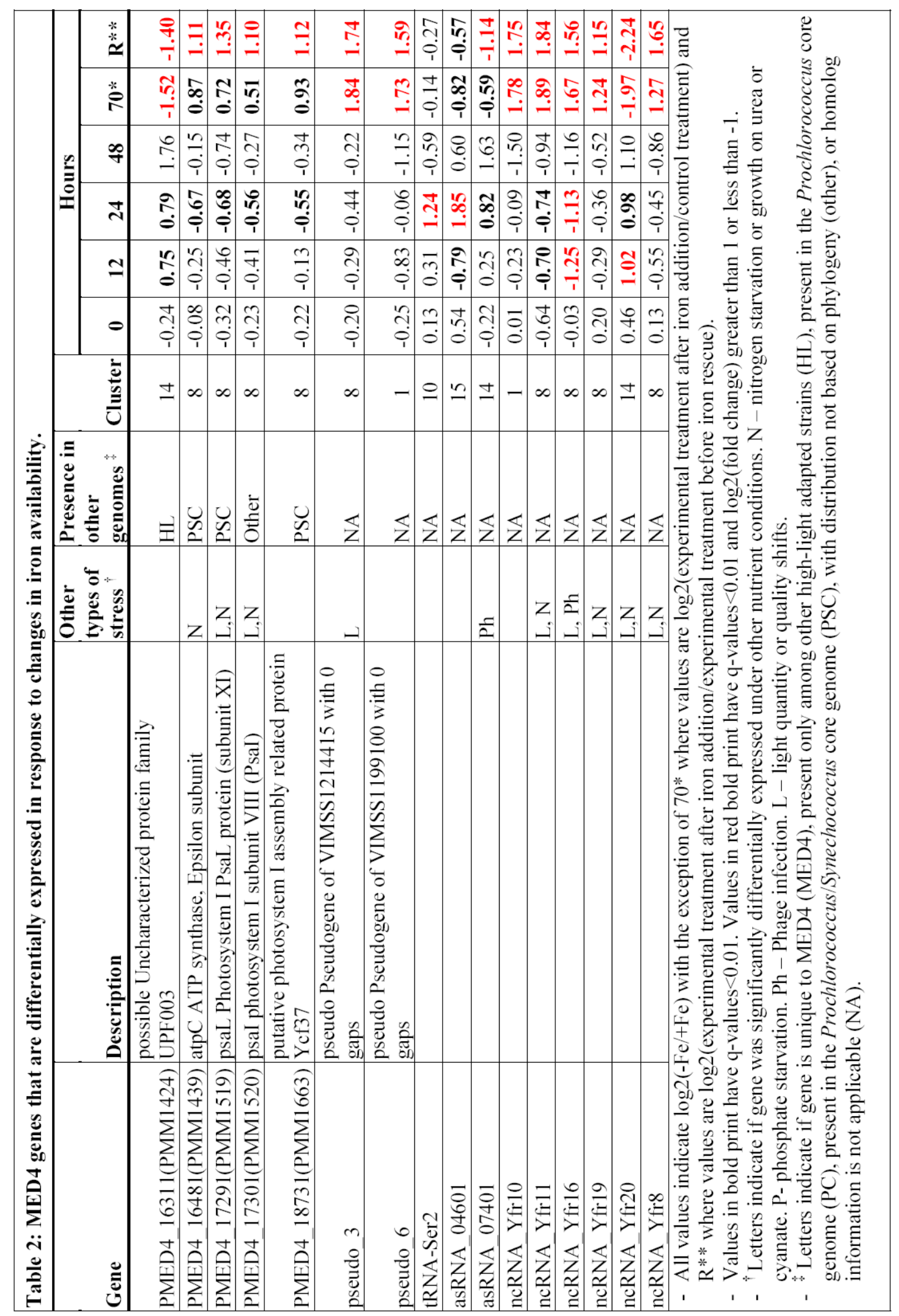




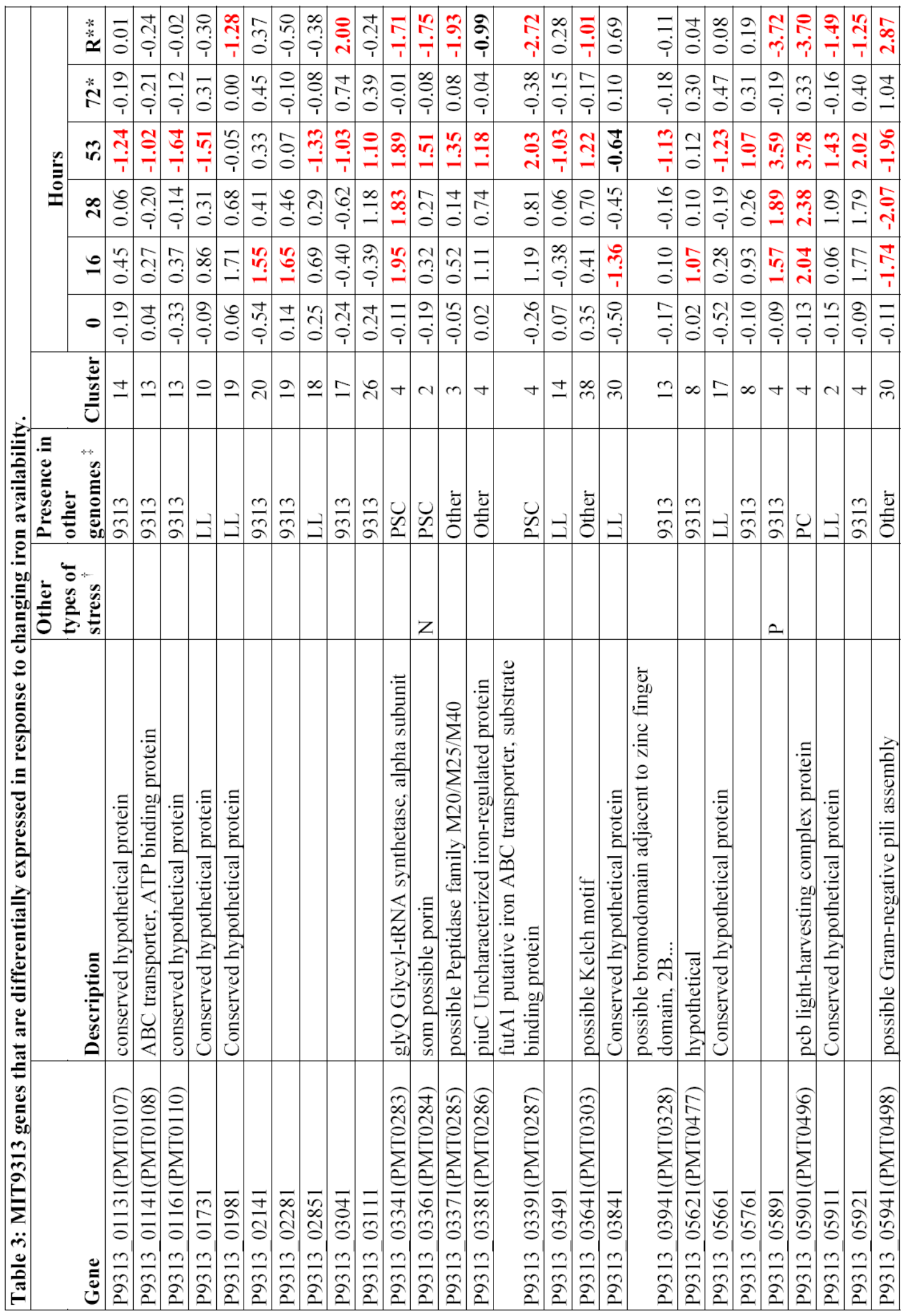




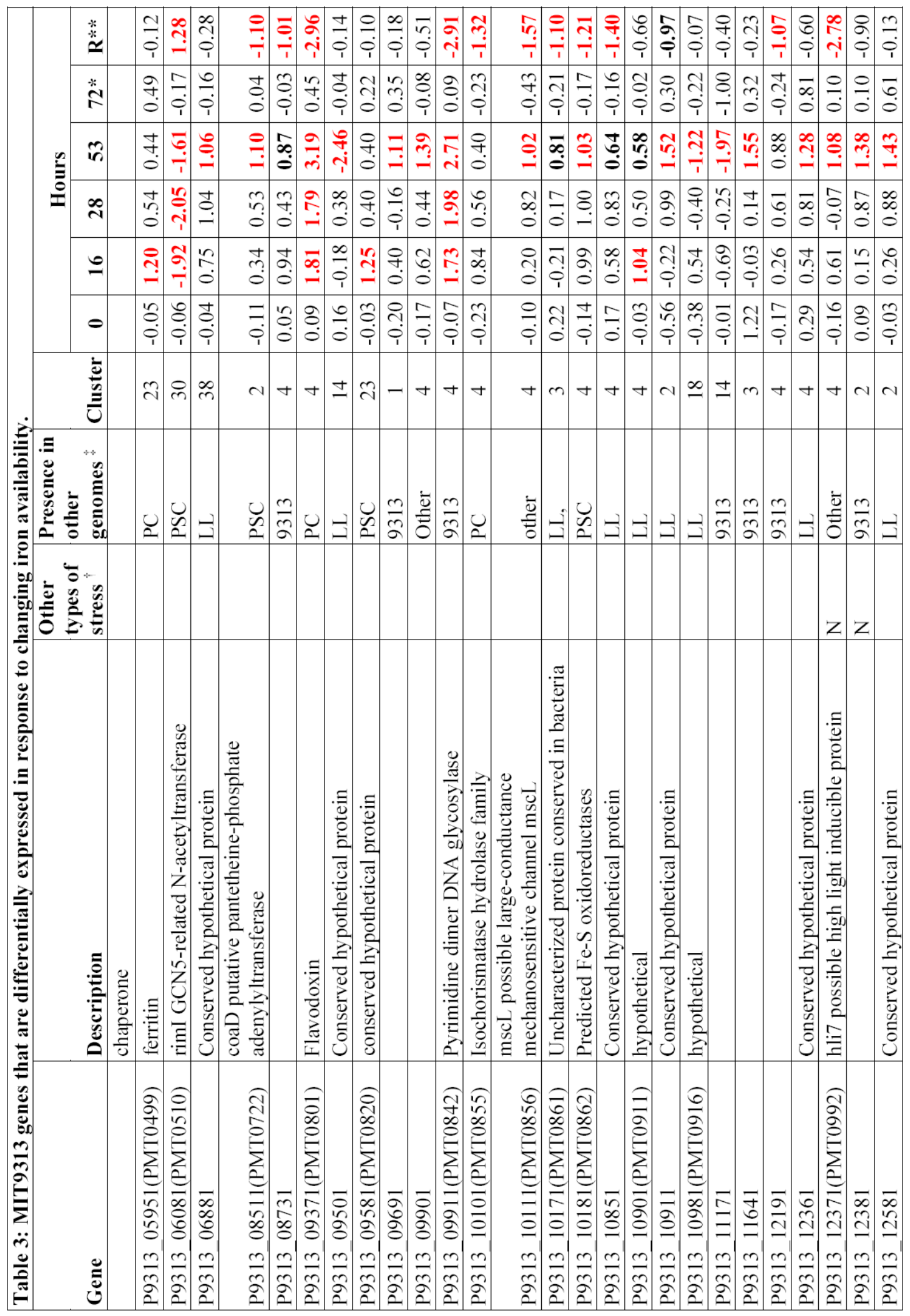




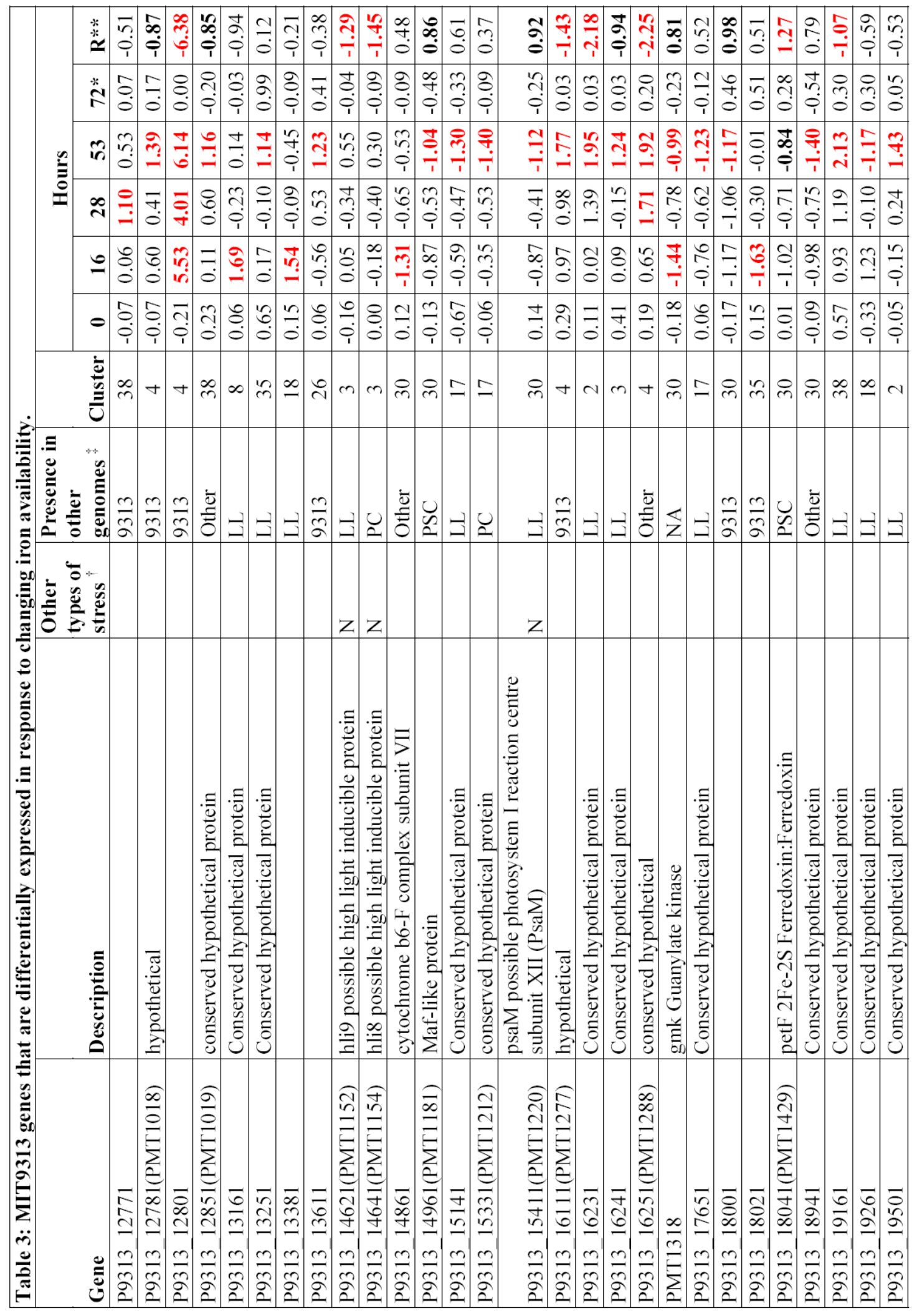




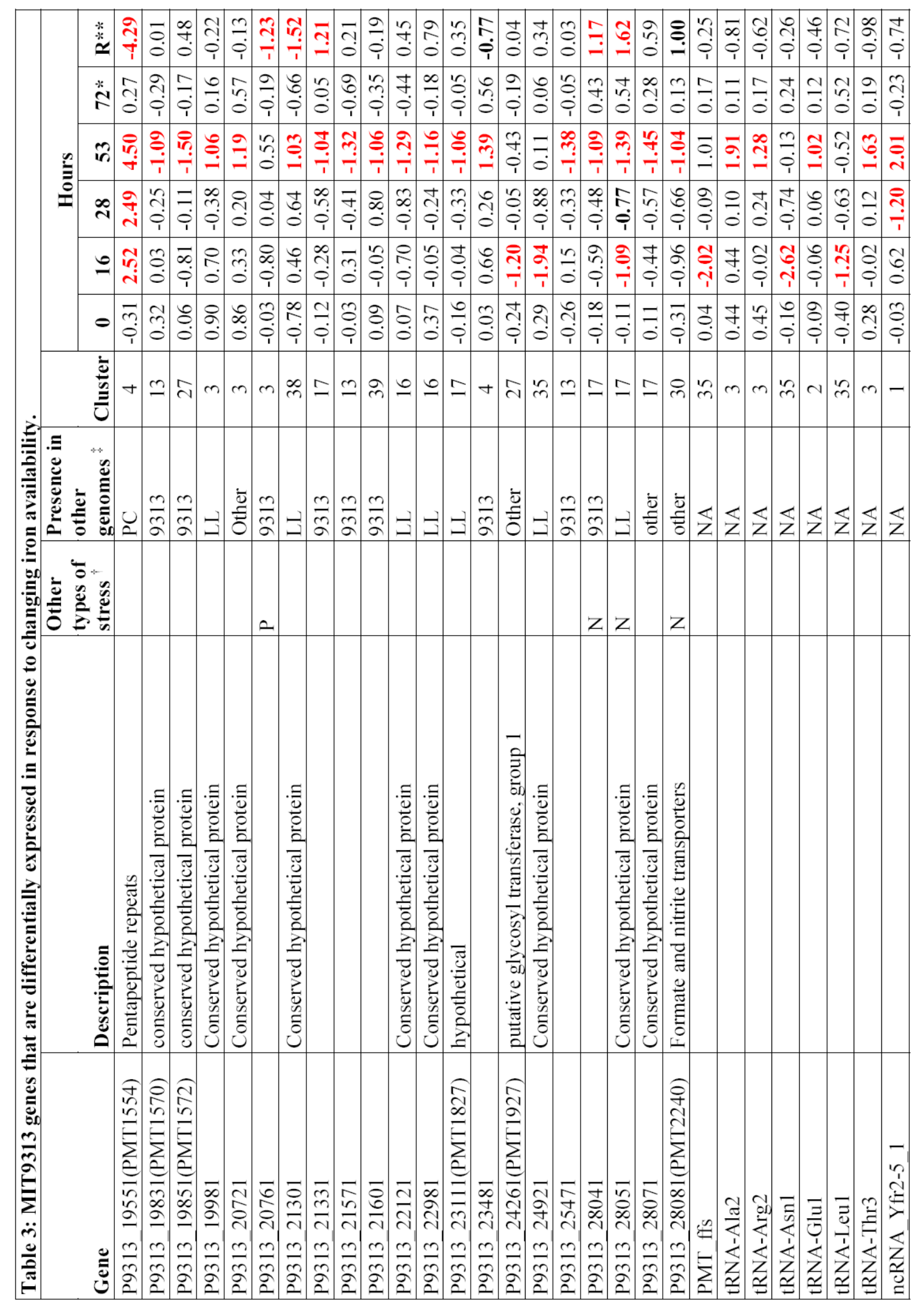




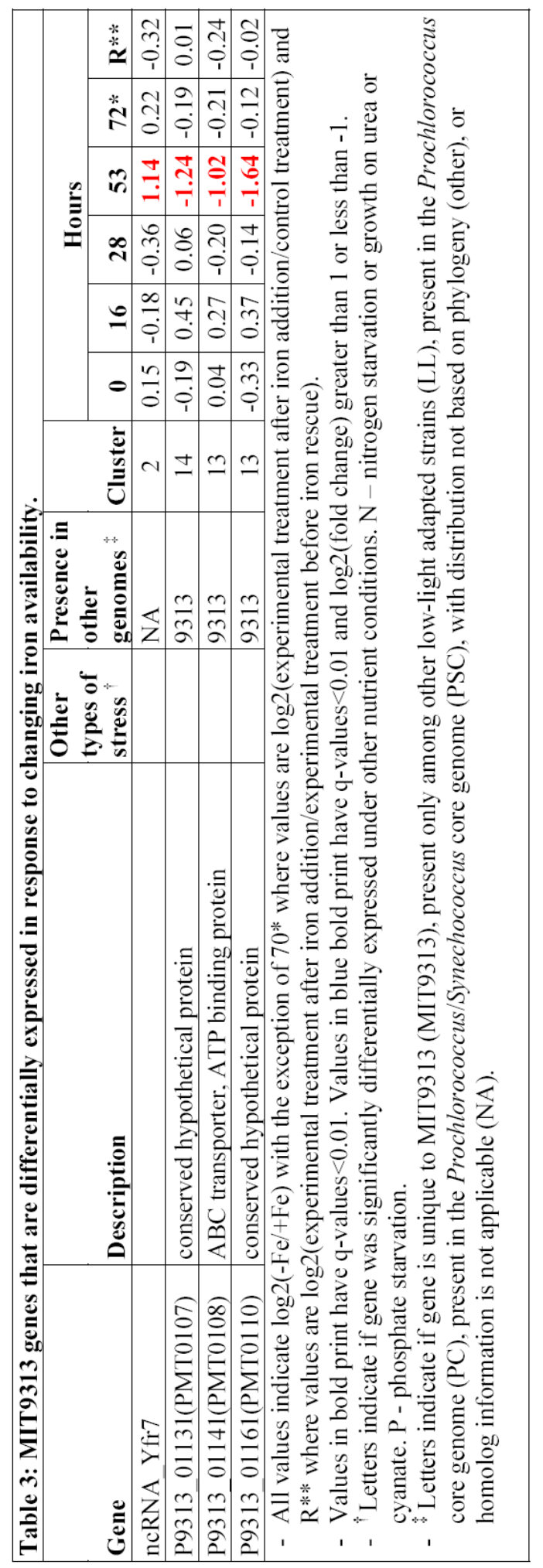




\section{References}

Alm EJ, Huang KH, Price MN, Koche RP, Keller K, Dubchak IL, Arkin AP (2005) The MicrobesOnline Web set for comparative genomics. Genome Research 15: 1015-1022.

Anbar AD, Knoll AH (2002) Proterozoic Ocean Chemistry and Evolution: A Bioinorganic Bridge? Science 297: 1137-1142.

Andrews SC, Robinson AK, Rodriguez-Quinones F (2003) Bacterial iron homeostasis. FEMS Microbiology Reviews 27: 215-237.

Baldi P, Long AD (2001) A Bayesian framework for the analysis of microarray expression data: regularized t -test and statistical inferences of gene changes. Bioinformatics 17: 509-519.

Beinert H, Holm RH, Munck E (1997) Iron-Sulfur Clusters: Nature's Modular, Multipurpose Structures. Science 277: 653-659.

Berman-Frank I, Cullen JT, Shaked Y, Sherrell RM, Falkowski PG (2001) Iron Availability, Cellular Iron Quotas, and Nitrogen Fixation in Trichodesmium. Limnology and Oceanography 46: 1249-1260.

Bibby TS, Mary I, Nield J, Partensky F, Barber J (2003) Low-light-adapted Prochlorococcus species possess specific antennae for each photosystem. Nature 424: 1051-1054.

Bibby TS, Nield J, Barber J (2001) Iron deficiency induces the formation of an antenna ring around trimeric photosystem I in cyanobacteria. Nature 412: 743-745.

Boekema EJ, Hifney A, Yakushevska AE, Piotrowski M, Keegstra W, Berry S, Michel KP, Pistorius EK, Kruip J (2001) A giant chlorophyll-protein complex induced by iron deficiency in cyanobacteria. Nature 412: 745-748.

Boyd PW, Jickells T, Law CS, Blain S, Boyle EA, Buesseler KO, Coale KH, Cullen JJ, de Baar HJW, Follows M, Harvey M, Lancelot C, Levasseur M, Owens NPJ, Pollard R, Rivkin RB, Sarmiento J, Schoemann V, Smetacek V, Takeda S, Tsuda A, Turner S, Watson AJ (2007) Mesoscale Iron Enrichment Experiments 1993-2005: Synthesis and Future Directions. Science 315: 612-617.

Buchanan SK, Smith BS, Venkatramani L, Xia D, Esser L, Palnitkar M, Chakraborty R, van der Helm D, Deisenhofer J (1999) Crystal structure of the outer membrane active transporter FepA from Escherichia coli. Nature Structural Biology 6: 56-63.

Busch A, Rimbauld B, Naumann B, Rensch S, Hippler M (2008) Ferritin is required for rapid remodeling of the photosynthetic apparatus and minimizes photo-oxidative stress in response to iron availability in Chlamydomonas reinhardtii. The Plant Journal 55: 201-211.

Butler YX, Abhayawardhane Y, Stewart GC (1993) Amplification of the Bacillus subtilis maf gene results in arrested septum formation. J Bacteriol 175: 3139-3145.

Cavender-Bares KK, Mann EL, Chisholm SW, Ondrusek ME, Bidigare RR (1999) Differential response of equatorial Pacific phytoplankton to iron fertilization. Limnology and Oceanography 44: 237-246.

Choe S, Boutros M, Michelson A, Church G, Halfon M (2005) Preferred analysis methods for Affymetrix GeneChips revealed by a wholly defined control dataset. Genome Biology 6: R16.

Coleman M, Sullivan M, Martiny A, Steglich C, Barry K, Delong E, Chisholm SW (2006) Genomic islands and the ecology and evolution of Prochlorococcus. Science 311: 1768 - 1770.

Coleman ML, Chisholm SW (2007) Code and context: Prochlorococcus as a model for cross-scale biology. Trends in Microbiology 15: 398-407.

Dos Santos PC, Dean DR (2008) A newly discovered role for iron-sulfur clusters. Proceedings of the National Academy of Sciences 105: 11589-11590.

Dufresne A, Salanoubat M, Partensky F, Artiguenave F, Axmann I, Barbe V, Duprat S, Galperin M, Koonin E, Le Gall F, Makarova K, Ostrowski M, Oztas S, Robert C, Rogozin I, Scanlan D, Tandeau de Marsac N, Weissenbach J, Wincker P, Wolf Y, Hess W (2003) Genome sequence of the cyanobacterium Prochlorococcus marinus SS120, a nearly minimal oxyphototrophic genome. Proceedings of the National Academy of Sciences 100: 10020 - 10025.

Duhring U, Axmann IM, Hess WH, Wilde A (2006) An internal antisense RNA regulates expression of the photosynthesis gene isiA. PNAS 103: 7054-7058.

Eisen MB, Spellman PT, Brown PO, Botstein D (1998) Cluster analysis and display of genome-wide expression patterns. Proceedings of the National Academy of Sciences of the United States of America 95: 14863-14868. 
Ernst JF, Bennett RL, Rothfield LI (1978) Constitutive expression of the iron-enterochelin and ferrichrome uptake systems in a mutant strain of Salmonella typhimurium. J Bacteriol 135: 928-934.

Falkowski PG, Raven JA (2007) Aquatic Photosynthesis. Malden, MA: Blackwell Science.

Ferguson AD, Chakraborty R, Smith BS, Esser L, van der Helm D, Deisenhofer J (2002) Structural Basis of Gating by the Outer Membrane Transporter FecA. Science 295: 1715-1719.

Ferguson AD, Hofmann E, Coulton JW, Diederichs K, Welte W (1998) Siderophore-Mediated Iron Transport: Crystal Structure of FhuA with Bound Lipopolysaccharide. Science 282: 2215-2220.

Ferreira F, Straus NA (1994) Iron deprivation in cyanobacteria. Journal of Applied Phycology 6: 199-210.

Fleming GR, Grondelle Rv (1997) Femtosecond spectroscopy of photosynthetic light-harvesting systems. Current Opinion in Structural Biology 7: 738-748.

Frias-Lopez J, Shi Y, Tyson GW, Coleman ML, Schuster SC, Chisholm SW, DeLong EF (2008) Microbial community gene expression in ocean surface waters. Proceedings of the National Academy of Sciences 105: 3805-3810.

Garcia-Fernandez JM, de Marsac NT, Diez J (2004) Streamlined Regulation and Gene Loss as Adaptive Mechanisms in Prochlorococcus for Optimized Nitrogen Utilization in Oligotrophic Environments. Microbiol Mol Biol Rev 68: 630-638.

Goldman SJ, Lammers PJ, Berman MS, Sanders-Loehr J (1983) Siderophore-Mediated Iron Uptake in Different Strains of Anabaena sp. Journal of Bacteriology 156: 1144-1150.

Hantke K (1981) Regulation of ferric iron transport in Escherichia coli K12: Isolation of a constitutive mutant. Molecular and General Genetics 182: 1432-1874.

Hernandez JA, Curatti L, Aznar CP, Perova Z, Britt RD, Rubio LM (2008) Metal trafficking for nitrogen fixation: NifQ donates molybdenum to NifEN/NifH for the biosynthesis of the nitrogenase FeMocofactor. Proceedings of the National Academy of Sciences 105: 11679-11684.

Hunter KA, Boyd PW (2007) Iron-binding ligands and their role in the ocean biogeochemistry of iron. Environmental Chemistry 4: 221-232.

Hutchins DA, Witter AE, Butler A, Luther III GW (1999) Competition among marine phytoplankton for different chelated iron species. Nature 400: 858-861.

Jeong W, Cha M-K, Kim I-H (2000) Thioredoxin-dependent Hydroperoxide Peroxidase Activity of Bacterioferritin Comigratory Protein (BCP) as a New Member of the Thiol-specific Antioxidant Protein (TSA)/Alkyl Hydroperoxide Peroxidase C (AhpC) Family. J Biol Chem 275: 2924-2930.

Johnson KS, Gordon RM, Coale KH (1997) What controls dissolved iron concentrations in the world ocean? Marine Chemistry 57: 137-161.

Johnson ZI, Zinser ER, Coe A, McNulty NP, Woodward EMS, Chisholm SW (2006) Niche Partitioning Among Prochlorococcus Ecotypes Along Ocean-Scale Environmental Gradients. Science 311: 1737-1740.

Jordan P, Fromme P, Witt HT, Klukas O, Saenger W, Krausz N (2001) Three-dimensional structure of

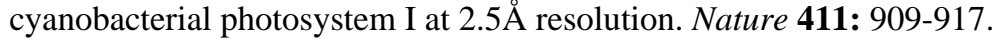

Katoh H, Grossman AR, Hagino N, Ogawa T (2000) A Gene of Synechocystis sp. Strain PCC 6803 Encoding a Novel Iron Transporter. Journal of Bacteriology 182: 6523-6524.

Katoh H, Hagino N, Grossman AR, Ogawa T (2001) Genes Essential to Iron Transport in the Cyanobacterium Synechocystis sp. Strain PCC 6803. Journal of Bacteriology 183: 2779-2784.

Keller MD, Bellows WK, Guillard RL (1988) Microwave treatment for sterilization of phytoplankton culture media. Journal of Experimental Marine Biology 117: 279-283.

Kettler GC, Martiny AC, Huang K, Zucker J, Coleman ML, Rodrigue S, Chen F, Lapidus A, Ferriera S, Johnson J, Steglich C, Church GM, Richardson P, Chisholm SW (2007) Patterns and Implications of Gene Gain and Loss in the Evolution of Prochlorococcus. PLoS Genetics 3: e231.

Kurisu G, Zhang H, Smith JL, Cramer WA (2003) Structure of the Cytochrome b6f Complex of Oxygenic Photosynthesis: Tuning the Cavity. Science 302: 1009-1014.

Levin PA, Margolis PS, Setlow P, Losick R, Sun D (1992) Identification of Bacillus subtilis genes for septum placement and shape determination. J Bacteriol 174: 6717-6728.

Lindell D, Jaffe JD, Coleman ML, Futschik ME, Axmann IM, Rector T, Kettler G, Sullivan MB, Steen R, Hess WR, Church GM, Chisholm SW (2007) Genome-wide expression dynamics of a marine virus and host reveal features of co-evolution. Nature 449: 83-86. 
Livak KJ, Schmittgen TD (2001) Analysis of Relative Gene Expression Data Using Real-Time Quantitative PCR and the 2-[Delta][Delta]CT Method. Methods 25: 402-408.

Mann EL, Chisholm SW (2000) Iron limits the cell division rate of Prochlorococcus in the eastern equatorial Pacific. Limnology and Oceanography 45: 1067-1076.

Martin JH, Fitzwater SE, Gordon RM (1990a) Iron deficiency limits phytoplankton growth in Antarctic waters. Global Biogeochemical Cycles 4: 5-12.

Martin JH, Fitzwater SE, Gordon RM, Hunter CN, Tanner SJ (1993) Iron, primary production and carbon nitrogen flux studies during the JGOFS North Atlantic bloom experiment. Deep-Sea Research 40: 115-134.

Martin JH, Gordon RM (1988) Northeast Pacific iron distributions in relation to phytoplankton productivity. Deep-Sea Research 35: 177-196.

Martin JH, Gordon RM, Fitzwater SE (1990b) Iron in Antarctic waters. Nature 345: 156-158.

Martin JH, Gordon RM, Fitzwater SE, Broenkow W (1989) VERTEX: phytoplankton/iron studies in the Gulf of Alaska. Deep-Sea Research 36: 649-680.

Martiny AC, Coleman ML, Chisholm SW (2006) Phosphate acquisition genes in Prochlorococcus ecotypes: Evidence for genome-wide adaptation. Proceedings of the National Academy of Sciences 103: 12552-12557.

Masse E, Gottesman S (2002) A small RNA regulates the expression of genes involved in iron metabolism in Escherichiacoli. Proceedings of the National Academy of Sciences 99: 4620-4625.

Masse E, Vanderpool CK, Gottesman S (2005) Effect of RyhB Small RNA on Global Iron Use in Escherichia coli. J Bacteriol 187: 6962-6971.

Meimberg K, Fischer N, Rochaix J-D, Muhlenhoff U (1999) Lys35 of PsaC is required for the efficient photoreduction of flavodoxin by photosystem I from Chlamydomonas reinhardtii. FEBS Journal 263: $137-144$.

Michel K, and Pistorius, E.K. (2004) Adaptation of the photosynthetic electron transport chain in cyanobacteria to iron deficiency: The function of IdiA and IsiA. Physiologia Plantarum 120: 3650 .

Michel K, Krüger, F., Pühler, A., and Pistorius, E.K. (1999) Molecular characterization of idiA and adjacent genes in the cyanobacteria Synechcoccus sp. strains PCC 6301 and PCC 7942. Microbiology 145: 1473-1484.

Michel K, Pistorius, E.K., and Golden, S.S. (2001) Unusual Regulatory Elements for Iron Deficiency Induction of the idiA Gene of Synechococcus elongatus PCC 7942. Journal of Bacteriology 183: 5015-5024.

Minasov G, Teplova M, Stewart GC, Koonin EV, Anderson WF, Egli M (2000) Functional implications from crystal structures of the conserved Bacillus subtilis protein Maf with and without dUTP. Proceedings of the National Academy of Sciences of the United States of America 97: 6328-6333.

Moore JK, Doney SC, Lindsay K (2004) Upper ocean ecosystem dynamics and iron cycling in a global three-dimensional model. Global Biogeochemical Cycles 18: GB4028.

Moore LM, Coe AC, Zinser ER, Saito MA, Sullivan MB, Lindell D, Frois-Moniz K, Waterbury W, Chisholm SW (2007) Culturing the marine cyanobacterium Prochlorococcus. Limnology and Oceanography Methods 5: 353-362.

Moore LR, Post AF, Rocap G, Chisholm SW (2002) Utilization of Different Nitrogen Sources by the Marine Cyanobacteria Prochlorococcus and Synechococcus. Limnology and Oceanography 47: 989-996.

Morel FMM, Hudson, R.J.M., and Price, N.M. (1991) Limitation of Productivity by Trace Metals in the Sea. Limnology and Oceanography 36: 1742-1755.

Morel MM, Price NM (2003) The Biogeochemical Cycles of Trace Metals in the Oceans. Science 300: 944-947.

Moseley JL, Allinger T, Herzog S, Hoerth P, Wehinger E, Merchant S, Hippler M (2002) Adaptation to Fedeficiency requires remodeling of the photosynthetic apparatus. European Molecular Biology Organization 21: 6709-6720.

Partensky F, Hess WR, Vaulot D (1999) Prochlorococcus, a Marine Photosynthetic Prokaryote of Global Significance. Microbiol Mol Biol Rev 63: 106-127. 
Posey JE, Gherardini FC (2000) Lack of a Role for Iron in the Lyme Disease Pathogen. Science 288: 16511653.

Price NM, Harrison GI, Hering JG, Hudson RJ, Nirel PMV, Palenik B, Morel FMM (1988) Preparation and chemistry of the artifical algal culture medium Aquil. Biological Oceanography 6: 443-461

Raven JA (1988) The Iron and Molybdenum use Efficiencies of Plant Growth with Different Energy, Carbon and Nitrogen Sources. New Phytologist 109: 279-287.

Rocap G, Larimer FW, Lamerdin J, Malfatti S, Chain P, Ahlgren NA, Arellano A, Coleman M, Hauser L, Hess WR, Johnson ZI, Land M, Lindell D, Post AF, Regala W, Shah M, Shaw SL, Steglich C, Sullivan MB, Ting CS, Tolonen A, Webb EA, Zinser ER, Chisholm SW (2003) Genome divergence in two Prochlorococcus ecotypes reflects oceanic niche differentiation. Nature 424: 1042-1047.

Rogers LJ (1987) Ferredoxins, flavodoxins and related proteins: structure, function and evolution. In The Cyanobacteria, P. Fay CVB (ed), pp 35-67. Amsterdam: Elsevier.

Rue EL, Bruland KW (1997) The Role of Organic Complexation on Ambient Iron Chemistry in the Equatorial Pacific Ocean and the Response of a Mesoscale Iron Addition Experiment. Limnology and Oceanography 42: 901-910.

Saito MA, Moffett JW, Chisholm SW, Waterbury JB (2002) Cobalt limitation and uptake in Prochlorococcus. Limnology and Oceanography 47: 1629-1636.

Saito MA, Sigman DM, Morel FMM (2003) The bioinorganic chemistry of the ancient ocean: the coevolution of cyanobacterial metal requirements and biogeochemical cycles at the ArcheanProterozoic boundary? Inorganica Chimica Acta 356: 308-318.

Sancho J (2006) Flavodoxins: sequence, folding, binding, function and beyond. Cellular and Molecular Life Sciences 63: 855-864.

Shaked Y, Kustka AB, Morel FMM (2005) A general kinetic model for iron acquisition by eukaryotic phytoplankton. Limnology and Oceanography 50: 872-882.

Singh AK, McIntyre LM, Sherman LA (2003) Microarray Analysis of the Genome-Wide Response to Iron Deficiency and Iron Reconstitution in the Cyanobacterium Synechocystis sp. PCC 6803. Plant Physiology 132: 1825-1839.

Steglich C, Futschik M, Rector T, Steen R, Chisholm SW (2006) Genome-Wide Analysis of Light Sensing in Prochlorococcus. J Bacteriol 188: 7796-7806.

Steglich C, Futschik ME, Lindell D, Voss B, Chisholm SW, Hess WR (2008) The Challenge of Regulation in a Minimal Photoautotroph: Non-Coding RNAs in Prochlorocococcus. PLoS Genetics 4: 1-14.

Storey JD, Tibshirani R (2003) Statistical significance for genomewide studies. Proceedings of the National Academy of Sciences of the United States of America 100: 9440-9445.

Straus NA (1994) Iron deprivation: physiology and gene regulation. In The Molecular Biology of Cyanobacteria, Bryant DA (ed), pp 731-750. Dordrecht, The Netherlands: Kluwer Academic Publishers.

Sunda W, Huntsman SA (1995) Iron uptake and growth limitation in oceanic and coastal phytoplankton. Marine Chemistry 50: 189-206.

Sunda W, Huntsman SA (2003) Effect of pH, light, and temperature on Fe-EDTA chelation and Fe hydrolysis in seawater. Marine Chemistry 84: 35-47.

Thayer MM, Ahern, H., Xing, D., Cunningham, R.P., Tainer, J.A. (1995) Novel DNA binding motifs in the DNA repair enzyme endonuclease III crystal structure. European Molecular Biology Organization 14: 4108-4120.

Tolonen AC, Aach J, Lindell D, Johnson ZI, Rector T, Steen R, Church GM, Chisholm SW (2006) Global gene expression of Prochlorococcus ecotypes in response to changes in nitrogen availability. Mol Syst Biol 2.

Tortell PD, Maldonado, M.T., Granger, J., and Price N.M. (1999) Marine bacteria and biogeochemical cycling of iron in the oceans. FEMS Microbiology Ecology 29: 1-11.

Touati D (2000) Iron and Oxidative Stress in Bacteria. Archives of Biochemistry and Biophysics 373: 1-6.

Webb EA, Moffett JW, Waterbury JB (2001) Iron Stress in Open-Ocean Cyanobacteria (Synechococcus, Trichodesmium, and Crocosphaera spp.): Identification of the IdiA Protein. Appl Environ Microbiol 67: 5444-5452. 
Wilderman PJ, Sowa NA, FitzGerald DJ, FitzGerald PC, Gottesman S, Ochsner UA, Vasil ML (2004) Identification of tandem duplicate regulatory small RNAs in Pseudomonas aeruginosa involved in iron homeostasis. Proceedings of the National Academy of Sciences of the United States of America 101: 9792-9797.

Wilhelm SW (1995) Ecology of iron-limited cyanobacteria: a review of physiological responses and implications for aquatic systems. Aquatic Microbial Ecology 9: 295-303.

Zinser ER, Coe A, Johnson ZI, Martiny AC, Fuller NJ, Scanlan DJ, Chisholm SW (2006) Prochlorococcus Ecotype Abundances in the North Atlantic Ocean As Revealed by an Improved Quantitative PCR Method. Appl Environ Microbiol 72: 723-732.

Zinser ER, Lindell D, Johnson ZI, Futschik ME, Steglich C, Coleman M, Wright MA, Rector T, Steen R, McNulty NP, Thompson LR, Chisholm SW (2009) Choreography of the Transcriptome, Photophysiology, and Cell Cycle of a Minimal Photoautotroph, Prochlorococcus. PLOS ONE.

Zurbriggen MD, Tognetti VB, Carrillo N (2007) Stress-inducible flavodoxin from photosynthetic microorganisms. The mystery of flavodoxin loss from the plant genome. Iubmb Life 59: 355-360. 


\title{
CHAPTER FOUR
}

Prochlorococcus iron physiology in the Eastern Equatorial Pacific and diversity of the iron stress induced gene, isiB

\author{
Anne W. Thompson ${ }^{1}$ \\ Maureen L. Coleman ${ }^{2}$ \\ Mak A. Saito ${ }^{3}$ \\ Sallie W. Chisholm ${ }^{2,4}$
}

\footnotetext{
${ }^{1}$ MIT-WHOI Joint Program in Biological Oceanography

${ }^{2}$ MIT Department of Biology

${ }^{3}$ Woods Hole Oceanographic Institution - Department of Marine Chemistry and Geochemistry

${ }^{4}$ MIT Department of Civil and Environmental Engineering
} 


\begin{abstract}
Prochlorococcus is numerically dominant in the vast oligotrophic gyres of the ocean and thus has the potential to strongly influence biogeochemical cycling in the upper ocean. As Prochlorococcus ecotypes adapt to the ocean environment, their diversity, abundance, and distribution is shaped by the dynamics of biogeochemical cycles. We understand how Prochlorococcus ecology is shaped by several factors including light, nitrogen, and phosphorus availability, yet we know little of the role iron plays in this complex system. Here, we test the iron physiology of natural assemblages of picocyanobacteria and find that Synechococcus is ironstressed in the same water samples where Prochlorococcus is not. In addition, we examine the diversity of the iron-stress induced gene, isiB, in sequenced Prochlorococcus and Synechococcus genomes as well as in environmental metagenomic databases and find a clear distinction between the two groups. The expression of isiB in response to iron deprivation in Prochlorococcus, its presence in the core genome, and abundance in the environment suggest that it may be useful as an indicator of iron stress in the field. Thus, we go on to design isiB primers for quantitative reverse-transcriptase PCR and describe future plans to quantify isiB transcription over natural gradients in iron availability in the ocean.
\end{abstract}

\title{
Introduction
}

Iron availability strongly influences the patterns and dynamics of primary productivity in the ocean as it facilitates electron transfer during photosynthesis. Global ecosystem models aim to bring together data on iron biogeochemical cycling and primary productivity both to evaluate our knowledge of what controls patterns in productivity and predict future scenarios of primary productivity (Johnson et al, 1997; Moore et al, 2004; Parekh et al, 2004; Parekh et al, 2005; Weber et al, 2007). Yet, the accuracy and predictive capabilities of these models are constrained by lack of data on species-specific iron requirements and the iron physiology of phytoplankton. In the last two decades, we have learned of the tremendous diversity of phytoplankton in the open ocean and with access to genome sequences and cultured isolates we are poised to understand the iron physiology of each. The cyanobacterium Prochlorococcus is abundant over the depth of the euphotic zone in the tropical and subtropical oceans of the globe and so fixes carbon dioxide and produces oxygen on global scales. Thus, determining the degree to which Prochlorococcus populations are iron-stressed would be a significant step towards informing the ecosystem models that aim to represent primary productivity in the ocean.

Measuring chlorophyll concentrations and cell abundances in response to iron addition is the traditional method of determining if field populations of phytoplankton are iron limited. Yet, this method is fraught with difficulties for an organism like Prochlorococcus that is quickly outgrown by and consumed by its grazers during nutrient enrichment bottle incubation experiments (Cavender-Bares et al, 1999; Coale et al, 1996). During IronExII, two studies were 
able to examine the specific response of Prochlorococcus to iron addition using two different methods. Both methods used flow cytometry to analyze the response of single cells to iron addition. Prochlorococcus red fluorescence per cell and forward angle light scatter (FALS) increased in the iron enrichment patch compared to populations outside the patch, indicating a release from iron-limitation (Cavender-Bares et al, 1999). Also, cell cycle analysis showed that the growth rate of Prochlorococcus cells increased from 0.6 day $^{-1}$ to 1.1 day $^{-1}$ over 6 days of iron enrichment to the patch (Mann and Chisholm, 2000). Changes in Prochlorococcus cell density did not suggest release from iron limitation in either experiment most likely because of increased grazing pressure following iron enrichment. Importantly, by examining physiology at the cellular level these experiments demonstrated that iron availability plays a role in Prochlorococcus ecology.

In the decade since the IronExII experiment, we have learned more about iron speciation in the ocean and the potential of iron speciation to influence primary productivity. Most of the iron in natural seawater appears to be complexed with organic ligands of broadly unknown origin and bioavailability (Hunter and Boyd, 2007; Rue and Bruland, 1995, 1997; Wu and Luther, 1995). Furthermore, colloidal and soluble size fractions of iron may have different patterns of distribution over depth suggesting different biological roles for each fraction of the total iron pool (Wu et al, 2001). Additionally, the photo-reduction of iron bound to organic ligands appears to have a significant influence on the concentrations of different iron species over a 24-hour lightdark period (Weber et al, 2007). Finally, improvements in the techniques used to measure trace metal concentrations now permit us to survey trace metal concentrations in the ocean with greater accuracy and resolution (Cullen et al, 2006; Johnson et al, 2006; Noble et al, 2008; Saito and Schneider, 2006). It will be important to understand how iron speciation and concentrations influence the ecology, distribution, and productivity of Prochlorococcus and other active or abundant marine phytoplankton.

The response of different Prochlorococcus ecotypes to iron availability in culture is also evidence of how iron influences Prochlorococcus ecology. We observe that the low-light adapted ecotype, MIT9313, is able to survive at lower iron concentrations than the high-light adapted ecotype MED4 (Chapter 2, Figure 4). In addition, we see that other environmental variable, such as light, influences the iron requirement of Prochlorococcus ecotypes in different ways (Chapter 2). Additionally, the influence of iron in Prochlorococcus ecology is imprinted on both the core and non-core genomes of Prochlorococcus (Chapter 3). Thus, Prochlorococcus is defined by and 
diversified by its iron metabolism. Though Prochlorococcus thrives in oligotrophic environments, we hypothesize that iron plays an important role in its activity in the natural environment.

The goal of this study was to develop a molecular indicator that could be used to diagnose iron stress in Prochlorococcus. To assess iron stress in Prochlorococcus, we have chosen to measure the transcription of the isiB gene, which encodes an iron-stress induced electron transfer protein that is associated with PSI and is present in the Prochlorococcus core genome (Kettler et al, 2007; Smillie, 1965). During iron-stress, isiB is up-regulated as a replacement to the iron-requiring electron transfer protein ferredoxin in Prochlorococcus and many other phototrophs (Bibby et al, 2003; Smillie, 1965). Among the Prochlorococcus core genes, $i s i B$ is one of three that responds quickly and strongly to changes in iron availability (Chapter 3). We anticipate that a molecular indicator of iron stress will be a more sensitive measure of the level of iron stress in Prochlorococcus than the physiological parameters of chlorophyll and cell size. Thus, we aim to use physiological measurements of iron stress detected via flow cytometry and gene expression to detect the degree to which Prochlorococcus is iron stressed over the horizontal, vertical, and temporal dimensions of its habitat in the open ocean.

Here, we describe the status of the progress we have made towards the goal of using isiB as an indicator of iron stress. We first describe bottle incubation experiments that were used to create different scenarios of iron-limitation using the fungal siderophore desferrioxamine-B (DFB) (Wells, 1999; Wells et al, 1994) and replete iron conditions by iron addition that will test the sensitivity of our method in detecting different degrees of iron stress. Next, we will describe the diversity of isiB sequences from environmental metagenomic databases and argue for the suitability of $i s i B$ as an indicator of iron stress in Prochlorococcus. Finally, we will describe the steps that remain in the development of a quantitative method of detecting isiB transcription in natural seawater samples.

At the time of this writing, we focus on the questions (1) Do Prochlorococcus and Synechococcus in the same water sample respond to iron addition in the same way? (2) Does the diversity of isiB sequences follow 16S/23S ITS phyologeny among Prochlorococcus and Synechococcus? With a tool to measure the levels of iron stress among natural assemblages of Prochlorococcus ecotypes in the future we hope to ask (1) How does Prochlorococcus iron stress change over a diel cycle of light availability? (2) Does light limitation with depth influence iron stress in Prochlorococcus? (3) At what iron concentration and in what type of iron speciation regime does Prochlorococcus experience iron stress? 


\section{Materials and Methods}

Sample collection - All samples were collected in coincidence with two full trace-metal sampling programs which took place July-August 2005 (KN182-5) and October-November 2005 (KN1829) aboard the R/V Knorr operated by the Woods Hole Oceanographic Institution. Samples for bottle incubations were collected by pumping seawater from $\sim 5$ meters depth into a trace-metal clean carboy in a positive-pressure clean area using a Teflon diaphragm pump and trace-metal clean tubing at the stations marked in Figure 1. The collection carboy was flushed for at least 5 minutes with the sample seawater before the seawater was reserved for experiments. RNA/DNA and flow cytometry samples were collected either by using a standard 24-bottle CTD-rosette deployed by cable, a trace-metal clean 6-bottle rosette (General Oceanics), a trace-metal clean single 10L bottle (General Oceanics) deployed on a Kevlar line, or from the bottle incubations.

On-deck bottle incubations - Bottle incubations were prepared in a positive-pressure trace metal clean area using trace-metal clean techniques. $\mathrm{FeSO}_{4}$ was added to a final concentration of $1 \mathrm{nM}$. Desferrioxamine B (DFB) was added to a final concentration of 20nM. The bottles used for the incubations were soaked overnight in 1\% citranox (Alconox), rinsed six times with Milli-Q water, then soaked for at least 3 days in $10 \% \mathrm{HCl}$ (Baker Instra-analyzed), then rinsed three times with pH 2 Milli-Q water prior to use. Single time-point incubations were conducted in $1.2 \mathrm{~L}$ polycarbonate bottles (Nalgene) in triplicate. Time-course incubations were conducted in $4 \mathrm{~L}$ polycarbonate bottles in duplicate and were sampled under trace-metal clean conditions. All

bottles were incubated together in a lidded transparent blue plexiglass box that was additionally shielded from light with black mesh to about $\sim 15 \%$ surface irradiance. The incubation box was constantly flushed with surface seawater to maintain a constant temperature. Bottles were transported in the dark from the incubators to the ship-based laboratory for sampling to prevent high-light shock.

Flow cytometry sampling - Samples for flow cytometry were collected from each incubationbottle either at the beginning and end of the incubation (single time-point incubations) or at regular intervals throughout the time-course (in the time-course incubations). Samples of $1 \mathrm{ml}$ 


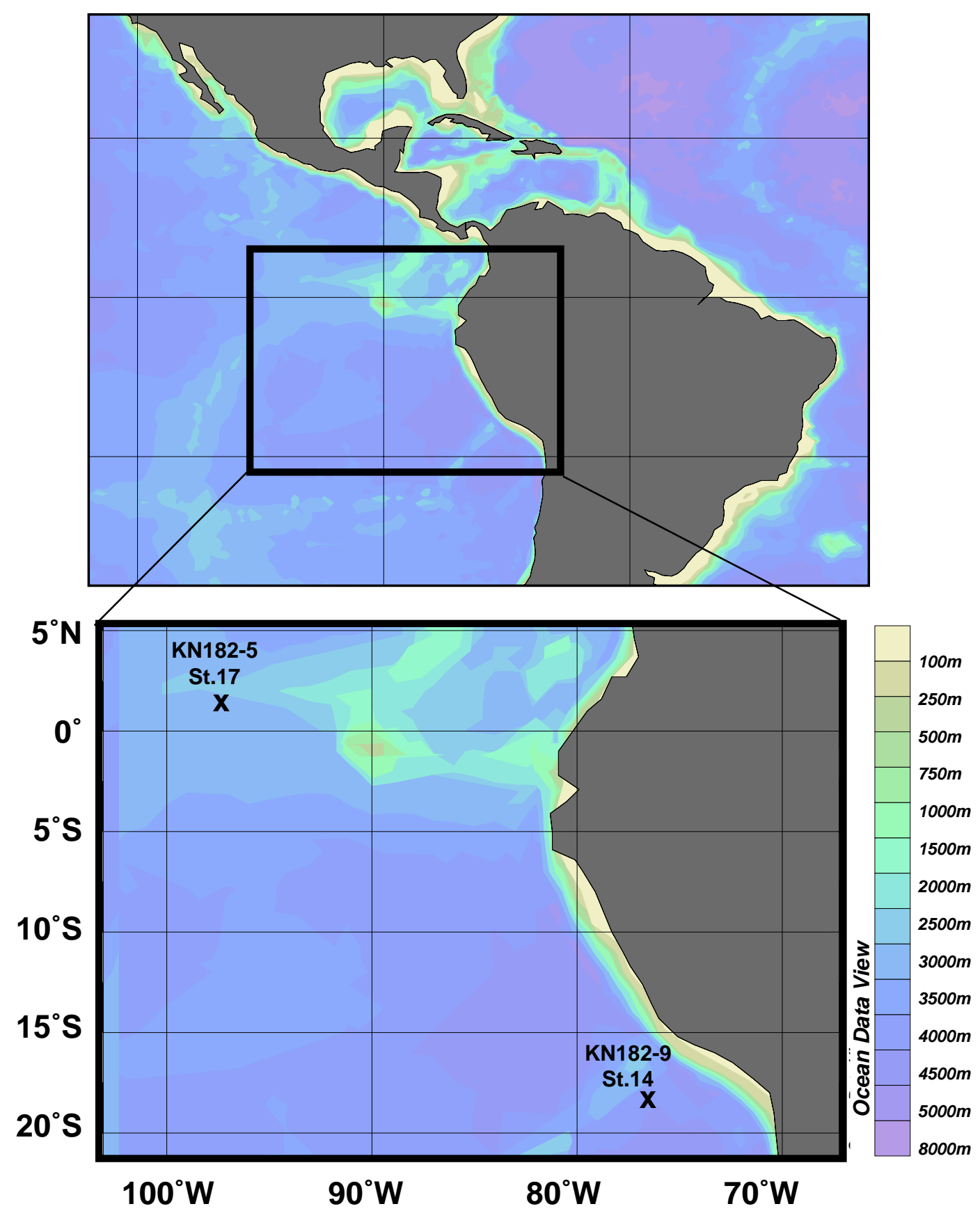

Figure 1: Station locations of bottle incubation experiments conducted on the KN182-5 cruise in July/August 2005 and the KN182-9 cruise conducted in October/November 2005. Station location are marked by “X”. Ocean Data View (http://odv.awi.de/) was used to create the maps. 
were fixed for 10 minutes in the dark in $0.12 \%$ glutaraldehyde (Tousmis), flash frozen in liquid nitrogen, and transported to the laboratory in a liquid-nitrogen dry shipper and stored at $-80^{\circ} \mathrm{C}$ until analysis. Red fluorescence per cell, forward scatter, and cell number were measured in comparison to $2 \mu \mathrm{m}$ FluorescBrite beads that were used as an internal standard (Polysciences, Inc) using an Influx cell sorter flow cytometer (Cytopeia).

Nucleic acid sampling - Seawater samples for nucleic acids were collected from the sample vessels (see above) in dark bottles to prevent exposure to light as soon after sampling as possible. Duplicate (triplicate if from an incubation conducted in triplicate) $1000 \mathrm{ml}$ volumes of seawater samples were in-line filtered through $25 \mathrm{~mm} 10 \mu \mathrm{m}$ polycarbonate filters then collected on $25 \mathrm{~mm}$ $0.2 \mu \mathrm{m}$ polyethersulfone filters (Supor) held in two separate plastic filter holders (Savillex) downstream of a multi-channel peristaltic pump (MasterFlex). Filters were immediately placed in $1 \mathrm{ml}$ RNAlater (Ambion) in $2 \mathrm{ml}$ bead-beatter tubes and stored at $4^{\circ} \mathrm{C}$ for 24 hours according the manufacturer's protocol. Samples were then moved to $-70^{\circ} \mathrm{C}$ until they were transported in liquidnitrogen dry-shippers from the ship to the laboratory where they were stored at $-80^{\circ} \mathrm{C}$ until analysis. Tubing, collection vessels, and filter holders were soaked in $10 \%$ bleach for at least one hour in between uses on different samples. Samples to test nucleic acid extraction methods were collected in the laboratory. 2-3ml volumes of dense Prochlorococcus cultures (strains MED4, MIT9313, MIT9312, and NATL1A) were vacuum-filtered onto $25 \mathrm{~mm} 0.2 \mu \mathrm{m}$ polyethersulfone filters (Supor) and handled the same way as the environmental samples thereafter.

Nucleic acid extractions -RNAlater preserved samples were thawed on ice and filters were removed from bead-beatter tubes, blotted on a fresh dust-free tissue (Kimberly Clark), and placed in a RNAse-free $1.5 \mathrm{ml}$ microfuge tube (Ambion) using forceps that were cleaned and treated with RNAseZap (Ambion) between samples. Filters were centrifuged for 1 minute at 5000rpm to remove excess RNAlater then placed back in the original bead-beatter tube that had been emptied of residual RNAlater and rinsed with two $1 \mathrm{ml}$ volumes of $10 \mathrm{mM}$ TrisHCl pH8 (Sigma). We then added $300 \mu 110 \mathrm{mM}$ TrisHCl $\mathrm{pH} 8$ to each tube and subjected each to 2 minutes of beating at 4800 rpm. $10 \mu 1$ of $300 \mathrm{mg} / \mathrm{ml}$ lysozyme (Sigma) and $10 \mu 1$ SuperaseIn (Ambion) were added to the sample. Each sample was vortexed then centrifuged quickly to collect fluid in the bottom of the tube. Samples were then incubated at $37^{\circ} \mathrm{C}$ for 60 minutes with the addition of a second aliquot of lysozyme and SuperaseIn at 30 minutes. Tubes were then placed on ice and centrifuged to collect 
liquid at the tube bottom. $300 \mu 1$ of homogenate was removed to a fresh $1.5 \mathrm{ml}$ RNAse-free microfuge tube (Ambion). Total nucleic acids were extracted using the MasterPure ${ }^{\mathrm{TM}}$ Complete DNA and RNA Purification Kit (Epicentre Biotechnologies) following the manufacturer's protocol for total nucleic acid purification protocol for fluid samples. Total nucleic acids were divided in half for separate DNA and RNA purification reactions which were conducted following the manufacturer's protocol. Extracted RNA and DNA were re-suspended in $20 \mu 1 \mathrm{TE}$ buffer. Concentrations of nucleic acids were measured using the Quant-iT ${ }^{\mathrm{TM}}$ RiboGreen ${ }^{\circledR} \mathrm{RNA}$ Assay Kit and Quant-iTTM PicoGreen ${ }^{\circledR}$ DNA Assay Kit.

Prochlorococcus and Synechococcus isiB phylogeny - Flavodoxin (isiB) sequences were gathered from several environmental sequence databases to assess the diversity of this gene among natural Prochlorococcus and Synechococcus populations. The MIT9301 isiB amino acid sequence was used as the query sequence because MIT9301-like cells are most abundant in the Global Ocean Survey (Rusch et al, 2007). We applied TBLASTN against the Global Ocean Survey dataset (Rusch et al, 2007) and several sets of whole fosmids and fosmid ends from the Hawaiian Ocean Time-Series Station ALOHA (Coleman et al, 2006; DeLong et al, 2006) to gather natural isiB sequences. Sequences were retained only if they showed greater than $40 \%$ similarity to the query with E-values less than 1e-20. We then used the best BLASTX hit to determine if each sequence most likely belonged to Prochlorococcus or Synechococcus. Sequences were aligned using ClustalW (Larkin et al, 2007) and the alignment was checked manually in BioEdit (http://www.mbio.ncsu.edu/BioEdit/bioedit.html). Neighbor-joining trees (1000 replicates for bootstrap analysis) were constructed using PHYLIP

(http://evolution.genetics.washington.edu/phylip.html). Synechocystis PCC6803 was used as the outgroup taxon for the analysis.

PCR primer design and optimization - In order to avoid the complications of using degenerate primers, we designed PCR primers to target four Prochlorococcus ecotypes, rather than a consensus sequence of all Prochlorococcus isiB sequences. Several primer sets were designed based on the sequences of isiB in each ecotype and include MED4/MIT9515 (eMED4), NATL1A/NATL2A (eNATL), MIT9313/MIT9301 (e9313), and MIT9312/MIT9215/MIT9301/AS9601 (e9312) using Genefisher 2.0 (Giegerich et al, 1996). Nucleotide alignments for each group were created in ClustalW (Larkin et al, 2007) are depicted 


\begin{abstract}
MIT_ 9301 AS $9 \overline{6} 01$ MIT9215

MIT 9301 AS $9 \overline{6} 01$

MIT9215

MIT 9312

MIT 9301

AS $9 \overline{6} 01$

MIT9215

MIT 9312

MIT 9301

AS $9 \overline{6} 01$

MIT 9215

MIT_9312

MIT 9301

AS $9 \overline{6} 01$

MIT9215

MIT_9312

MIT 9301

AS $9 \overline{6} 01$

MIT9215

MIT_9312

MIT 9301

AS $9 \overline{6} 01$

MIT9215

MIT_ 9312

MIT 9301

AS $9 \overline{6} 01$

MIT9215

MIT 9312

MIT 9301

AS $9 \overline{6} 01$

MIT9215

MIT 9312

ATGACTGTAGGAATTTATTACGCAACTACAACCGGAAAAACAGAAGATGTGGCTGATCGT 60 ATGACTGTAGGAATTTATTACGCAACTACAACTGGAAAAACTGAAGACGTAGCAGATCGT 60 ATGACTGTAGGAATTTATTACGCAACTACAACCGGAAAAACTGAAGACGTAGCTGATCGT 60 ATGACTGTAGGAATTATTACGCAACTACAACTGGAAAAACTGAAGACGTAGCTGATCGT 60 $* * * * * * * * * * * * * * * * * * * * * * * * * * * * * * * * * * * * * * * * * * * * * * * * * * * * * * *$

CTTCACAACTTTATCTCTTCAGCTGAAGCACCTAAAGATGTATCTGATGTGGATGATCTT 120 CTTCACAACTTTATTTCTTCAGCAGAAGCACCTAAAGATGTATCTGATGTAGATGATCTT 120 CTTCACAACTTTATTTCTTCAGCAGAATCACCTAAAGATGTATCTGATGTAGATGATCTT 120 CTTCATAACTTTATTCTTCGGCAGAAGCACCTAAAGATGTATCTGATGTTGATGATCTT 120

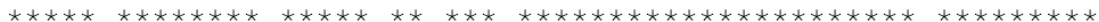

TCAGAATTTGAGGGTCTTGATGGAATTATCTGCGGGATACCCACTTGGAATACTGGTGCC 180 TCAGAATTTGAAGGTCTTGATGGAATTATCTGTGGGATACCTACATGGAATACAGGCGCC 180 TCAGAATTTGAAGGTCTTGATGGAATTATCTGTGGCATTCCTACTTGGAATACTGGTGCG 180 TCAGAATTCGAAGGTCTTGATGGAATTATCTGCGGGATACCTACTTGGAACACTGGGGCT 180

$* * * * * * * * * * * * * * * * * * * * * * * * * * * * * * * * * * * * * * * * * * * * * * * * *$

GATGAAGAAAGGTCGGGAACTGCATGGGATTCAATCTTAGAGGATATTGGTGAACTAAGT 240 GATGAAGAAAGATCGGGAACTGCATGGGATTCAATCTTAGAGGATATTGGTGAGCTAAGT 240 GATGAAGAAAGGTCAGGAACTGCATGGGATTCAATTTTAGAGGATATTGGTGAATTAAGT 240 GATGAAGAAAGATCAGGAACTGCATGGGATTCAATTCTGGAGGATATTGGTGAACTAAGT 240 $* * * * * * * * * * * * * * * * * * * * * * * * * * * * * * * * * \quad * * * * * * * * * * * * * * * \quad * * * * *$

TTATCAGGAAAAAAAGTTGCAATTTTGGTTTAGGAGATTCTTCTACATATACAGAAAAC 300 TTATCAGGAAAAAAGGTTGCGATTTTTGGTTTAGGAGATTCTTCTACATATACAGAAAAC 300 TTATCAGGGAAAAAAGTTGCAATTTTTGGTTTAGGAGATTCTTCTACCTACACAGAAAAC 300 TTATCAGGAAAAAAGGTTGCAATTTTTGGTTTAGGAGATTCTTCTACCTACACAGAAAAC 300 $* * * * * * * * * * * * * * * * * * \quad * * * * * * * * * * * * * * * * * * * * * * * * * * * * * * * * * * * * *$

TATTGTGATGCAATGGAAGAACTTCATAGCTACTTCACAAAAGCAGGCGCCGAAATGGTC 360 TATTGTGATGCTATGGAAGAACTTCATAGCTACTTCACAAAAGCAGGCGCCGAAATGGTC 360 TATTGTGATGCAATGGAAGAGCTTCATAGCTACTTCATTAAAGCAGGTGCAGAAATGGTA 360 TATTGTGATGCCATGGAAGAACTTCACAGCTACTTCGCTAAAGCAGGTGCCGAAATGGTC 360

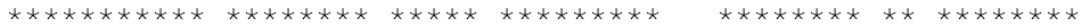

GGTTACGTAGATAAATCTTCTTATACATTTGATGAATCCAAAAGTGTTATGGGGGACAGA 420 GGTTACGTAGATAAATCTTCTTATACATTTGATGAGTCTAAAAGTGTAATTGGAGAAAGC 420 GGTTATGTAGATAAATCTACTTATACATTTGATGAATCTAAAAGTGTTATTGGAGAAAGC 420 GGTTACGTAGATAAATCTTCTTATACGTTTGATGAATCTAAAAGTATCATTGGAGAAAGC 420

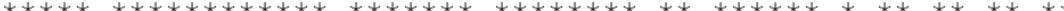

TTTTGTGGATTACCTCTTGATGAGGATAGTGAATCTGATTTGACCGATTCGCGTCTTGAG 480 TTTTGTGGATTACCTCTTGATGAGGATAGTGAATCAGATTTGACCGATTCTCGTCTTGAA 480 TTTTGCGGATTACCTCTTGATGAGGATAGTGAATCCGATTTGACCGATTCACGTCTTGAA 480 TTTTGTGGATTACCTCTTGATGAGGATAGTGAATCTGATTTAACCGATACACGTCTTGAA 480

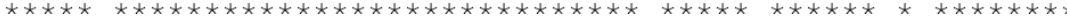

ACATGGGCTTCTCAGCTTAAGGGTGAAATCCCCTCATTGGCATAG 525 ACATGGGCTTCTCAGCTTAAGGGTGAAATCCCCTCATTGGCGTAA 525 ACATGGGCTTCTCAGCTTAAGGGTGAAATTCCCGCATTGGCGTAA 525 ACATGGGCTTCTCAGCTTAAGGACGAAATCCCTTCATTGGCGTAA 525

$* * * * * * * * * * * * * * * * * * * * * * \quad * * * * * * * \quad * * * * * * * * *$
\end{abstract}

\title{
Figure 2: (A) e9312 isiB alignment used to design primers (Table 1). Asterisks mark nucleotides that are conserved in all sequences.
}




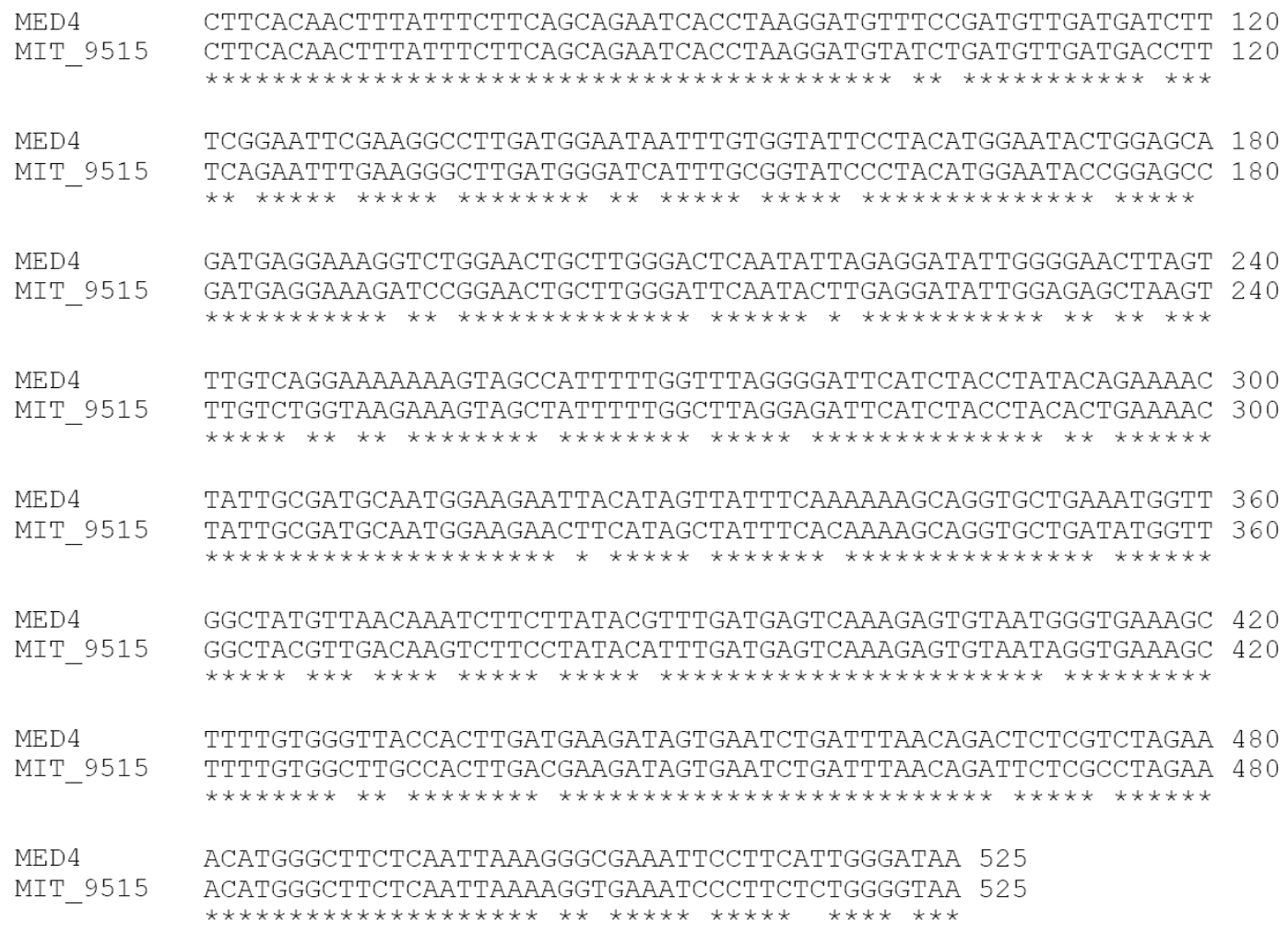

Figure 2: (B) eMED4 isiB alignment used to design primers (Table 1). Asterisks mark nucleotides that are conserved in all sequences. 


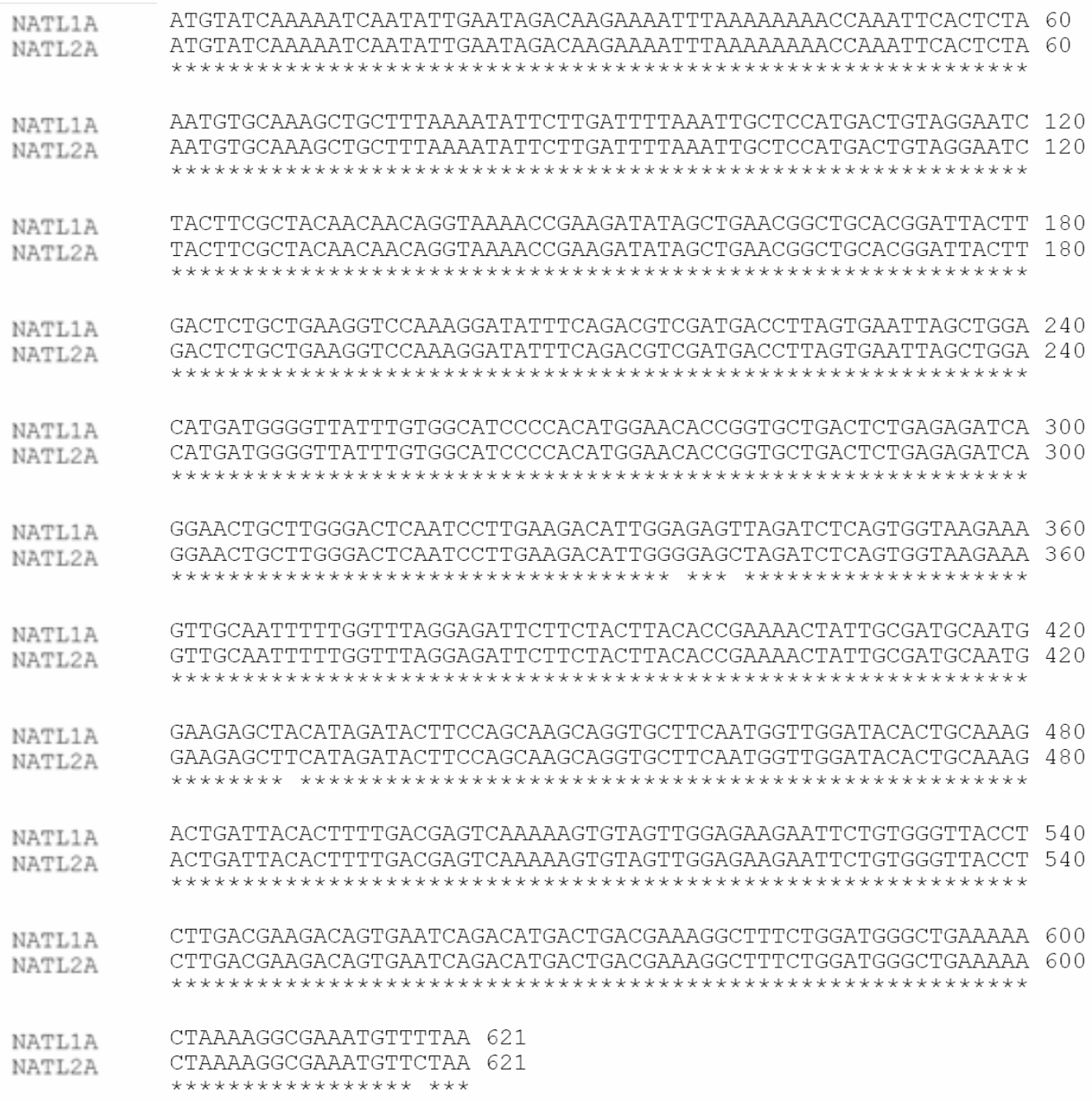

Figure 2: (C) eNATL isiB alignment used to design primers (Table 1). Asterisks mark nucleotides that are conserved in all sequences. 


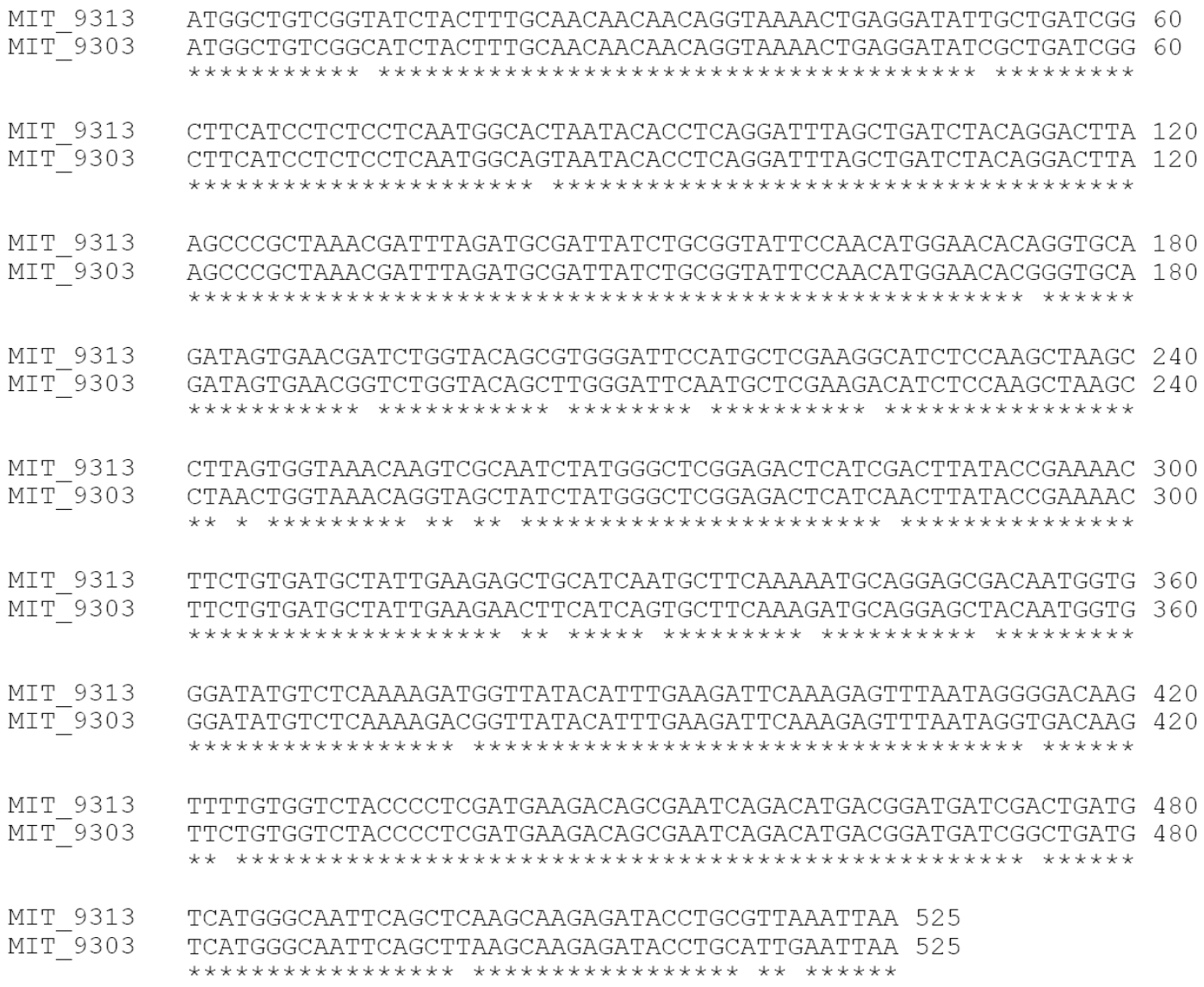

Figure 2: (D) e9313 isiB alignment used to design primers (Table 1). Asterisks mark nucleotides that are conserved in all sequences. 
in Figure 2. DNA from one sequenced strain of each ecotype group was chosen as template in the optimization of the many primer sets that were designed for each ecotype. DNA for PCR primer optimization was extracted from cultures of MED4, MIT9313, MIT9312, and NATL1A obtained from the Chisholm Laboratory culture collection using the nucleic acid protocol described above. Primer optimization PCR reactions were conducted with 100pg template DNA and a final concentration of $0.4 \mu \mathrm{M}$ of each primer supplemented with the Quantitect SYBR Green PCR master mixture (QIAGEN Inc., Valencia, CA) to $25 \mu 1$ reaction volumes. A DNA Engine Opticon (MJ Research Inc.) was used for the PCR reactions. We used an annealing temperature gradient of $50^{\circ} \mathrm{C}$ to $60^{\circ} \mathrm{C}$ across the columns of a 96 -well plate to determine the optimal annealing temperatures for each primer set based on the quality of its quantification and melting curves. One primer set was chosen for each ecotype that efficiency amplified the target sequence and did not efficiency amplify a product from the other template DNA (Table 1).

\begin{tabular}{|l|l|l|l|l|l|}
\hline Table 1: Best candidates for flavodoxin (isiB) PCR primers in Prochlorococcus. \\
\hline $\begin{array}{l}\text { Template/ } \\
\text { target }\end{array}$ & Primer name & $\begin{array}{l}\text { Primer sequence } \\
\text { (5' to 3') }\end{array}$ & $\begin{array}{l}\text { Conc. } \\
(\mathbf{\mu M})\end{array}$ & $\begin{array}{l}\mathbf{T}_{\mathbf{a}} \text { opt. } \\
\left({ }^{\circ} \mathbf{C}\right)\end{array}$ & $\begin{array}{l}\text { Size } \\
\text { (bp) }\end{array}$ \\
\hline $\begin{array}{l}\text { MED4/ } \\
\text { eMED4 }\end{array}$ & eMED4_isiB3 & $\begin{array}{l}\text { F: GTA GGA ATT TAT TAC GCA AC } \\
\text { R: ACA TCC TTA GGT GAT TCT }\end{array}$ & 0.4 & 51.7 & 191 \\
\hline $\begin{array}{l}\text { MIT9313/ } \\
\text { e9313 }\end{array}$ & e9313_isiB3 & $\begin{array}{l}\text { F: TAA TAC ACC TCA GGA TTT AGC } \\
\text { R: AGG CTT AGC TTG GAG ATG }\end{array}$ & 0.4 & 54.3 & 159 \\
\hline $\begin{array}{l}\text { MIT9312/ } \\
\text { e9312 }\end{array}$ & ev_isiB1 & $\begin{array}{l}\text { F: CAC CTA AAG ATG TAT CTG ATG } \\
\text { R: GCA TCA CAA TAG TTT TCT G }\end{array}$ & 0.4 & 50.3 & 223 \\
\hline $\begin{array}{l}\text { NATL1A/ } \\
\text { eNATL }\end{array}$ & eNATL_isiB3 & $\begin{array}{l}\text { F: GGC TGC ACG GAT TAC TTG } \\
\text { R: GAA TCT CCT AAA CCA AAA ATT GC }\end{array}$ & 0.4 & 56 & 223 \\
\hline
\end{tabular}

\section{Results and Discussion}

Effect of DFB on natural Prochlorococcus and Synechococcus populations - Our physiological studies in MED4ax and MIT9313ax demonstrate that decreased red fluorescence per cell and reduced cell size are manifestations of iron stress (Chapter 2 Figure 2 and Chapter 3 Figure 2). Addition of DFB to natural seawater induced decreases in cell size and red fluorescence per cell in both Prochlorococcus and Synechococcus populations at two separate sites in the Eastern Equatorial Pacific (Figure 3 and 4). These results give us confidence that the microbial communities in bottle incubations treated with DFB will represent iron-stressed populations for testing the sensitivity of the proposed isiB indicator of iron stress. The ability of DFB to induce the symptoms of iron limitation or stress in natural populations of phytoplankton in this study is consistent with the hypothesis that it induces iron stress in other marine phytoplankton (Wells, 1999; Wells et al, 1994). Also, iron stress of Prochlorococcus cultures and field populations has 

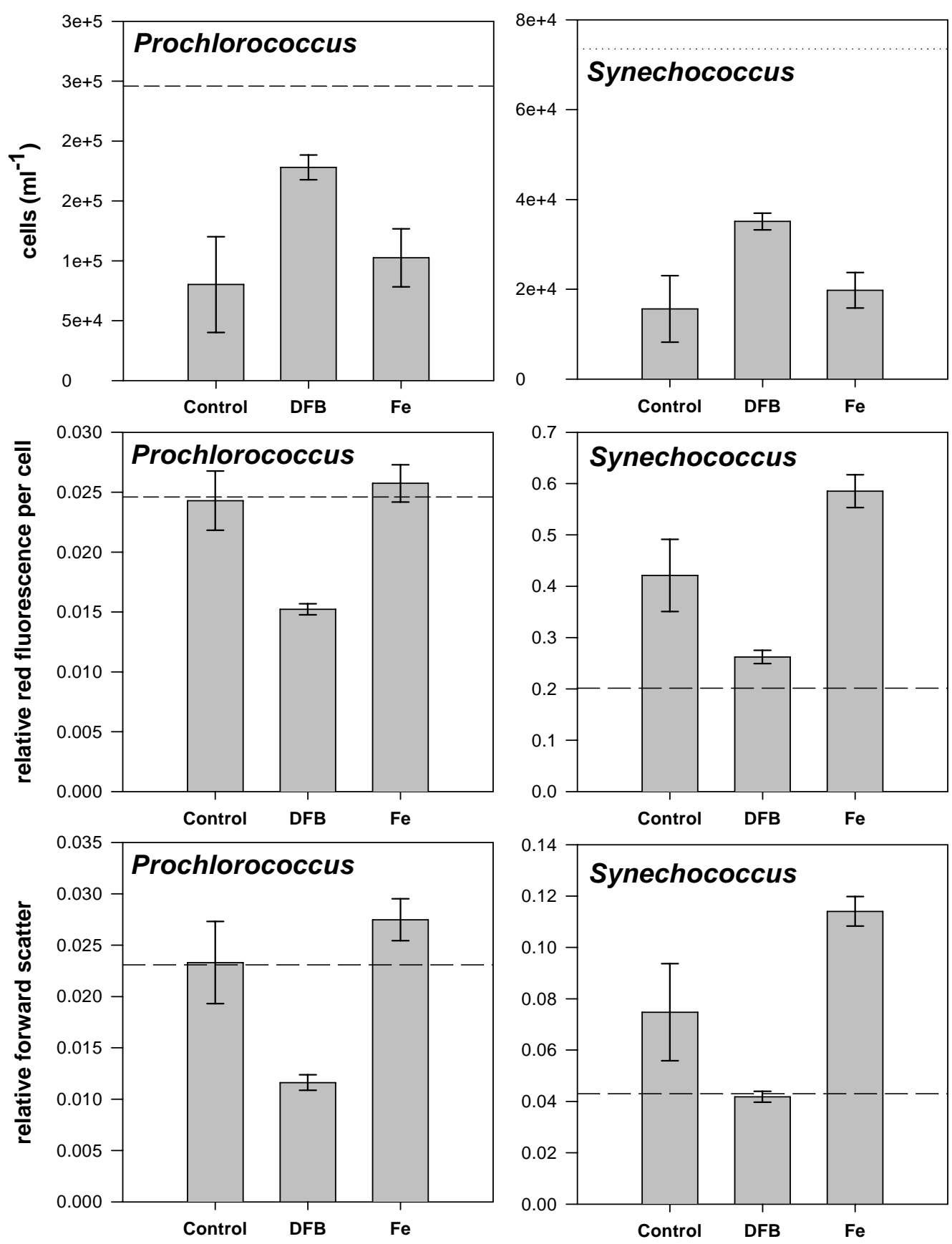

Figure 3: Single time-point bottle incubation experiments conducted at Station 17 in August 2005 during the KN182-5 cruise. Samples were harvested after a 72hour incubation. Dashed lines indicate cellular parameters of Prochlorococcus and Synechococcus before the start of the incubation. Red fluorescence per cell and forward scatter are relative to standard $2 \mu \mathrm{m}$ fluorescent beads and represent triplicate incubations for each treatment. 

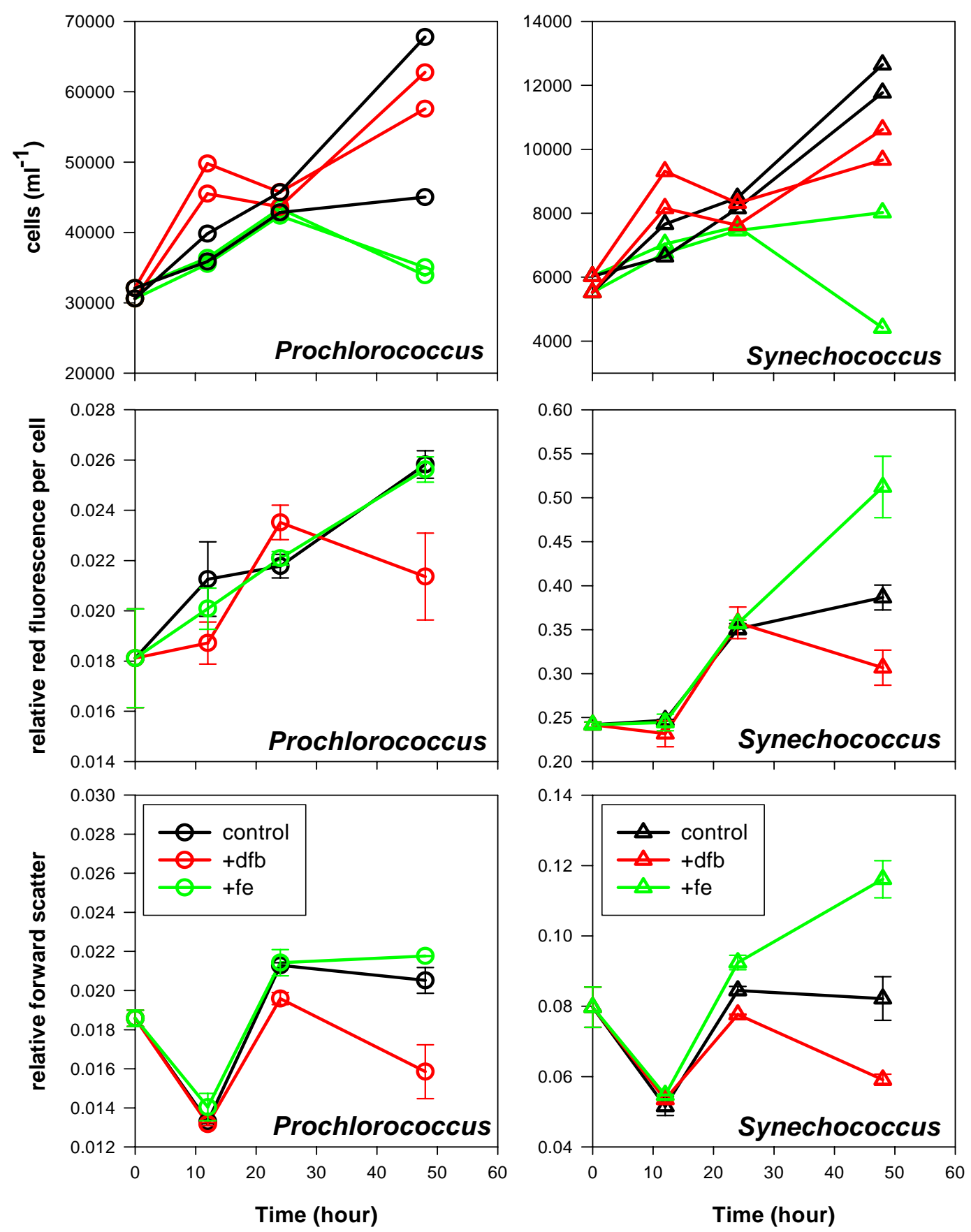

Figure 4: Time-course bottle incubation experiments conducted at Station 14 in October 2005 during the KN182-9 cruise. Cell numbers, red fluorescence per cell and forward scatter represent duplicate incubations for each treatment and red fluorescence and forward scatter are relative to standard $2 \mu \mathrm{m}$ fluorescent beads. All sampling was conducted under positive-pressure trace-metal clean conditions. 
been shown to induce decreases in red fluorescence per cell (a proxy for chlorophyll per cell) and forward scatter (a proxy for cell size), which is similar to the effect of DFB shown here (Cavender-Bares et al, 1999).

Addition of DFB also caused an increase final cell concentration of both Prochlorococcus and Synechococcus in incubation experiments conducted at Station 17 in the Equatorial Pacific (Figure 3). And, an initial increase in cell concentration was induced by DFB in the time-course DFB-addition experiment conducted in an oligotrophic regime off the coast of Peru on cruise KN182-9 (Figure 4). Without cell-cycle analysis over a 24-hour period to measure cell division rates we cannot assume the increase in yield is due to an increase in growth rate of Prochlorococcus or Synechococcus because of the possible top-down influences on cell yield (Mann et al, 2000). Yet, we are intrigued by the changes in the microbial community that allow a significant increase in the cell concentration of marine picocyanobacteria populations in response to DFB addition.

Iron stress of co-occurring Prochlorococcus and Synechococcus - Bottle incubations from two sites in the Eastern Equatorial Pacific show that in the same water sample, Synechococcus responds to iron addition more dramatically than Prochlorococcus (Figure 3 and 4). Over 48 hours, red fluorescence per cell stays the same for Prochlorococcus populations in the control and iron addition treatments while it significantly increases in the Synechococcus population of the iron addition treatment. Similarly, forward angle light scatter increases markedly in the iron addition bottles relative to the control bottles for the Synechococcus population but only slightly for the Prochlorococcus population. These observations are intriguing because the two populations of cells co-exist in the same physical and chemical conditions.

One possible explanation of why we observe that Synechococcus and Prochlorococcus adapted to the same in situ conditions respond differently to iron addition is due to differences in iron metabolism between the two groups. We have already seen that there are significant differences in iron physiology, i.e. minimum iron concentration required for growth, among the Prochlorococcus ecotypes alone (Chapter 2 and 3). Cell size is an important parameter that could influence the iron physiology of Prochlorococcus and Synechococcus as it is for other phytoplankton species. For example, when normalized to cell surface area, coastal and open ocean species of diatoms, dinoflagellates, and coccolithophores show iron limitation at the same concentrations of iron (Sunda and Huntsman, 1995). Therefore, we could hypothesize that 
Synechococcus is more iron-limited than Prochlorococcus in our incubations due to its larger cell size and lower surface area to volume ratio, thus less efficient iron acquisition relative to requirement, and so responds significantly to iron addition while Prochlorococcus does not. It is also possible that Prochlorococcus has a lower Fe: $\mathrm{C}$ ratio, or a smaller iron requirement, than Synechococcus. So, another reasonable hypothesis is that the red fluorescence per cell and cell size of Prochlorococcus are already at maximum levels given the available light. A third hypothesis focuses on differences between Prochlorococcus and Synechococcus related to iron acquisition. Through growth experiments and analysis of whole-genome expression in response to iron stress we see that iron contributes to the diversity among the Prochlorococcus ecotypes. For instance, MIT9313 can grow at lower iron concentrations than MED4 (Chapter 2). And, we see that most of the iron-responsive genes in both strains are not in the Prochlorococcus core genome (Chapter 3). Also, we see that only a few of the iron-responsive genes of either strain are present in Synechococcus genomes (Chapter 3, Figure 9). Therefore, we believe it is possible that Prochlorococcus uses higher affinity iron transporters or a different system of iron acquisition than Synechococcus and so has access to a greater portion of the total iron pool while Synechococcus maintains sub-maximal concentrations of chlorophyll per cell and cell size because it cannot acquire enough iron.

The kinetics of the response to iron addition could also explain the difference bewteen the Synechococcus and Prochlorococcus response to iron addition. In the final time-point of the incubation (Figure 4), Prochlorococcus in the iron-addition treatment has slightly more red fluorescence per cell than the control, which may represent the beginning of a response to the iron addition. Possibly, the iron-sensing system of Synechococcus is more sensitive to changes in iron availability and elicits a quicker response that allows this group to take advantage of the newly available iron before a similar response is manifested in Prochlorococcus. In culture, Prochlorococcus responds quickly to iron-addition at the transcriptional level but an increase in red fluorescence per cell occurs more slowly, especially in MED4 (Chapter 3, Figure 2). A longer time-course incubation experiment would be appropriate to test this hypothesis as well as testing the kinetics of the response of Prochlorococcus and Synechococcus to iron deprivation in the laboratory.

$i s i B$ diversity - Our original goal was to develop a method of quantifying isiB expression in natural populations of Prochlorococcus and Synechococcus. To this end we had to design primers 
Figure 5: Neighbor-joining tree showing the diversity among Prochlorococcus and Synechococcus flavodoxin (isiB) sequences from environmental databases. 1000 replicate trees were used to determine bootstrap values. Pink text marks the isiB sequences of cultured isolates of Synechococcus. Green text marks the isiB sequences of cultured isolates of Prochlorococcus. Pink line shows the clustering of Synechococcus isiB sequences and the green line shows where Prochlorococcus isiB sequences cluster.

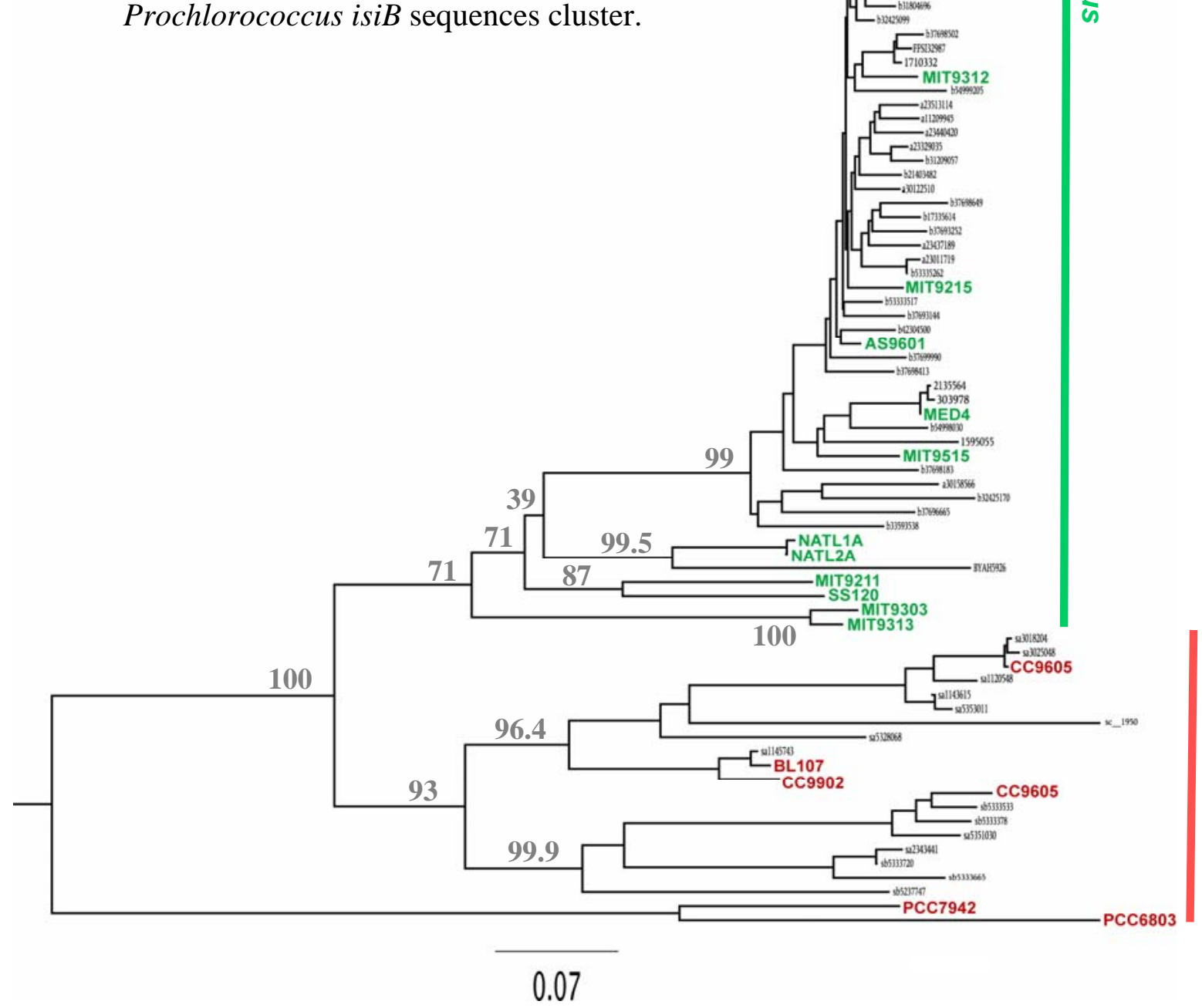


that would distinguish between the isiB sequences of these two groups of marine picocyanobacteria. So, we assessed the phylogeny of isiB from the natural environment and from cultured isolates and found that it is consistent with phylogeny based on 16S/23S ITS (Rocap et al, 2002) (Figure 5). The sequences of Prochlorococcus and Synechococcus isiB genes have diverged distinctly and isiB phylogeny also follows the high-light and low-light adapted ecotype distinction in Prochlorococcus. This result suggests that the isiB gene has been transferred between marine cyanobacteria via vertical gene transfer rather than through horizontal gene transfer. We hypothesize that $i s i B$ is not important in adaptations to local iron regimes but is a central component of the iron metabolism of open-ocean Prochlorococcus.

The phylogenetic distinction between isiB sequences in Prochlorococcus and Synechococcus and its presence in the Prochlorococcus core genome make isiB expression measurements useful to characterize iron stress in Prochlorococcus and Synechococcus. We propose the development of a quantitative method to measure the number of isiB mRNA transcripts normalized to isiB gene copy numbers using quantitative RT-PCR and PCR, respectively, among the mixed community in natural seawater samples. Starting with controlled bottle-incubation experiments we will test the ability of our method to detect the relative levels of iron stress in Prochlorococcus and Synechococcus populations in DFB-treated samples compared to samples where iron has been added and to an intermediate no-addition control. Next, we will be able to describe how Prochlorococcus iron physiology changes in the natural environment in response to natural patterns of iron availability that change with geography, depth, and time. We hope that the elucidation of how iron availability influences the ecology of Prochlorococcus and Synechococcus will be informative to ecosystem models that aim reconstruct the patterns and dynamics of marine primary productivity based on the data that is available.

\section{Next steps}

Testing the specificity of isiB primers - With the goal of studying iron stress in natural Prochlorococcus and Synechococcus populations, it is necessary to make sure our isiB primers are specific to either their Prochlorococcus or Synechococcus targets. We plan to obtain DNA from the Synechococcus genomes that contain isiB including CC9605, CC9902, BL107, and CC9311 (microbesonline.org) and attempt to amplify the isiB gene using each of the Prochlorococcus isiB primer sets at their optimal conditions determined by testing against Prochlorococcus DNA (Table 1). Primers that efficiently amplify both Prochlorococcus and 
Synechococcus isiB sequences will be discarded and new primers will be developed to replace them.

Additionally, before using the isiB primers developed in this study to quantify gene expression in the field, we plan to make a clone library of the sequences that are amplified by each primer set from natural samples of seawater. Samples have been collected from several stations and depths on the KN182-5 cruise to the Eastern Equatorial Pacific in July 2005 and from Station 14 of the KN182-9 cruise to the Peru Upwelling Regime for this purpose. Gene products will be amplified for cloning using re-conditioning PCR (Thompson et al, 2002 ). The gene product in 100 clones from each primer set will be sequenced. Sequences from the clone library, environmental metagenomic databases, and cultured isolates will be aligned and used in the construction of another isiB phylogenetic tree. We will assess the specificity of the primers by noting whether primer sets amplify targets that cluster best with the isiB sequences of cultured isolates for which the primer set was designed (Table 1).

Testing the sensitivity of isiB as an iron stress indicator - The ability of our method to detect different degrees of iron stress in natural Prochlorococcus populations will be tested using DNA/RNA collected from the bottle-incubations discussed here (Figure 3 and 4). In each set of bottle-incubations, we used DFB to induce iron stress and iron addition to create iron-replete conditions. The control from each experiment will represent an intermediate condition of iron stress. As described in the methods section, nucleic acids will be extracted from samples taken from the bottle incubations. The copy number of isiB will be detected with quantitative PCR performed on the DNA fraction. mRNA transcripts will be quantified using RT-PCR on the RNA fractions. In this way, we will obtain a measure of the expression of isiB relative to its potential for expression in each sample and will understand the sensitivity of the method that will allow its application to un-amended samples from the environment.

How does iron stress change over the diel cycle?-At KN182-5 Station 11, we collected RNA/DNA samples at about $\sim 6$ hour intervals over a 26 -hour period at four depths within the upper euphotic zone to examine isiB expression over the diel cycle. In culture, isiB expression changes dramatically over a diel cycle with peak expression at about mid-day (Chapter 3 - Figure 8a). We hypothesize that the diel expression pattern in the field will match the expression pattern over the diel cycle in culture. As cells require more photosystems for efficient light-harvesting at 
depth, we expect a higher background level of isiB expression in the non-surface samples and smaller changes in the amplitude of isiB expression cycles over the diel period to match the muted shifts in irradiance at depth over the diel cycle.

Does light limitation with depth influence iron stress in Prochlorococcus? - Other studies have hypothesized that Prochlorococcus and other cells would be simultaneously iron- and lightlimited at depth (Bibby et al, 2003; Sunda and Huntsman, 1997; Zinser et al, 2009). This hypothesis is based on the involvement of iron in light-harvesting complexes and the importance of efficient light-harvesting in cells acclimated or adapted to low-light conditions. At several stations on both the KN182-5 cruise and the KN182-9 cruise we have collected RNA/DNA samples at many depths within the euphotic zone. We are excited to test the iron-light hypothesis by measuring the level of iron stress in Prochlorococcus populations over the depth of the euphotic zone.

At what iron concentration and in what iron speciation regime does Prochlorococcus become iron stressed? - Growth experiments of Prochlorococcus show that cells in culture are less capable of growth at low iron concentrations than natural populations of Prochlorococcus in the Equatorial Pacific (Chapter 2 - Figure 6). This result inspires the question: What is the iron concentration that induces iron stress in field populations of Prochlorococcus and Synechococcus? Surface samples taken from the KN182-5 and KN182-9 cruises will allow us to compare iron concentration and iron speciation data to iron stress in Prochlorococcus to answer this question. Furthermore, we observed that total iron concentrations appear more relevant in predicting the concentration of iron that limits Prochlorococcus growth in the field as opposed to concentrations of Fe' (Chapter 2 - Figure 6). Thus, measuring Prochlorococcus iron stress relative to iron speciation will allow us insight into the pool of iron that is most relevant in predicting the growth rate and iron physiology of marine picocyanobacteria in the natural environment.

\section{Acknowledgements}

A.W.T. is grateful to members of the Saito Laboratory for support with field work on the KN1825 cruise and is grateful to James Moffett for supporting participation on the KN182-9 cruise.

Tyler Goepfert deserves special thanks for facilitating the setup of trace metal clean space and 
technical support at sea during the KN182-5 and KN182-9 cruises. We also thank the Captain and crew of the R/V Knorr. Maureen Coleman was indispensable for her role in writing scripts to extract sequences from the environmental databases as was Paul Berube for assistance with phylogenetic analysis of sequences. Sébastien Rodrigue generously assisted with advice for the design and optimization of the PCR primers. Conversations with Eric Webb were extremely valuable in deciding on normalization methods for quantitative gene expression from field populations.

\section{References}

Bibby TS, Mary I, Nield J, Partensky F, Barber J (2003) Low-light-adapted Prochlorococcus species possess specific antennae for each photosystem. Nature 424: 1051-1054.

Cavender-Bares KK, Mann EL, Chisholm SW, Ondrusek ME, Bidigare RR (1999) Differential response of equatorial Pacific phytoplankton to iron fertilization. Limnology and Oceanography 44: 237-246.

Coale KH, Johnson KS, Fitzwater SE, Gordon RM, Tanner S, Chavez FP, Ferioli L, Sakamoto C, Rogers P, Millero F, Steinberg P, Nightingale P, Cooper D, Cochlan WP, Landry MR, Constantinou J, Rollwagen G, Trasvina A, Kudela R (1996) A massive phytoplankton bloom induced by an ecosystem-scale iron fertilization experiment in the equatorial Pacific Ocean. Nature 383: 495501.

Coleman ML, Sullivan MB, Martiny AC, Steglich C, Barry K, DeLong EF, Chisholm SW (2006) Genomic Islands and the Ecology and Evolution of Prochlorococcus. Science 311: 1768-1770.

Cullen JT, Bergquist BA, Moffett JW (2006) Thermodynamic characterization of the partitioning of iron between soluble and colloidal species in the Atlantic Ocean. Marine Chemistry 98: 295-303.

DeLong EF, Preston CM, Mincer T, Rich V, Hallam SJ, Frigaard N-U, Martinez A, Sullivan MB, Edwards R, Brito BR, Chisholm SW, Karl DM (2006) Community Genomics Among Stratified Microbial Assemblages in the Ocean's Interior. Science 311: 496-503.

Giegerich R, Meyer F, Schleiermacher C (1996) GeneFisher - software support for the detection of postulated genes. Proceedings of the International Conference of Intelligent Systems for Molecular Biology 4: 68-77.

Hunter KA, Boyd PW (2007) Iron-binding ligands and their role in the ocean biogeochemistry of iron. Environmental Chemistry 4: 221-232.

Johnson K, Boyle EA, Bruland KW, Measures C, Moffett JW (2006) Sampling and Analysis of Fe: The SAFE Iron Intercomparison Cruise. In Eos Trans. AGU pp Abstract xxxxx-xx. Ocean Science Meeting Supplement.

Johnson KS, Gordon RM, Coale KH (1997) What controls dissolved iron concentrations in the world ocean? Marine Chemistry 57: 137-161.

Kettler GC, Martiny AC, Huang K, Zucker J, Coleman ML, Rodrigue S, Chen F, Lapidus A, Ferriera S, Johnson J, Steglich C, Church GM, Richardson P, Chisholm SW (2007) Patterns and Implications of Gene Gain and Loss in the Evolution of Prochlorococcus. PLoS Genetics 3: e231.

Larkin MA, Blackshields G, Brown NP, Chenna R, McGettigan PA, McWilliam H, Valentin F, Wallace IM, Wilm A, Lopez R, Thompson JD, Gibson TJ, Higgins DG (2007) Clustal W and Clustal X version 2.0. Bioinformatics 23: 2947-2948.

Mann EL, Chisholm SW (2000) Iron limits the cell division rate of Prochlorococcus in the eastern equatorial Pacific. Limnology and Oceanography 45: 1067-1076.

Moore JK, Doney SC, Lindsay K (2004) Upper ocean ecosystem dynamics and iron cycling in a global three-dimensional model. Global Biogeochemical Cycles 18: GB4028.

Noble AE, Saito MA, Maiti K, Benitez-Nelson CR (2008) Cobalt, manganese, and iron near the Hawaiian Islands: A potential concentrating mechanism for cobalt within a cyclonic eddy and implications 
for the hybrid-type trace metals. Deep Sea Research Part II: Topical Studies in Oceanography 55: 1473-1490.

Parekh P, Follows M, Boyle EA (2004) Modeling the global ocean iron cycle. Global Biogeochemical Cycles 18.

Parekh P, Follows M, Boyle EA (2005) Decoupling of iron and phophate in the global ocean. Global Biogeochemical Cycles 19.

Rocap G, Distel DL, Waterbury JB, Chisholm SW (2002) Resolution of Prochlorococcus and Synechococcus Ecotypes by Using 16S-23S Ribosomal DNA Internal Transcribed Spacer Sequences. Appl Environ Microbiol 68: 1180-1191.

Rue EL, Bruland KW (1995) Complexation of iron(III) by natural organic ligands in the Central North Pacific as determined by a new competitive ligand equilibration/adsorptive cathodic stripping voltammetric method. Marine Chemistry 50: 117-138.

Rue EL, Bruland KW (1997) The Role of Organic Complexation on Ambient Iron Chemistry in the Equatorial Pacific Ocean and the Response of a Mesoscale Iron Addition Experiment. Limnology and Oceanography 42: 901-910.

Rusch DB, Halpern AL, Sutton G, Heidelberg KB, Williamson S, Yooseph S, Wu D, Eisen JA, Hoffman JM, Remington K, Beeson K, Tran B, Smith H, Baden-Tillson H, Stewart C, Thorpe J, Freeman J, Andrews-Pfannkoch C, Venter JE, Li K, Kravitz S, Heidelberg JF, Utterback T, Rogers Y-H, Falc, \#243, n LI, Souza V, Bonilla-Rosso G, \#225, Eguiarte LE, Karl DM, Sathyendranath S, Platt T, Bermingham E, Gallardo V, Tamayo-Castillo G, Ferrari MR, Strausberg RL, Nealson K, Friedman R, Frazier M, Venter JC (2007) The Sorcerer II Global Ocean Sampling Expedition: Northwest Atlantic through Eastern Tropical Pacific. PLoS Biology 5: e77.

Saito MA, Schneider DL (2006) Examination of precipitation chemistry and improvements in precision using the $\mathrm{Mg}(\mathrm{OH}) 2$ preconcentration inductively coupled plasma mass spectrometry (ICP-MS) method for high-throughput analysis of open-ocean Fe and Mn in seawater. Analytica Chimica Acta 565: 222-233.

Smillie RM (1965) Isolation of Phytoflavin, A Flavoprotein with Chloroplast Ferredoxin Activity. Plant Physiol 40: 1124-1128.

Sunda W, Huntsman SA (1995) Iron uptake and growth limitation in oceanic and coastal phytoplankton. Marine Chemistry 50: 189-206.

Sunda W, Huntsman SA (1997) Interrelated influence of iron, light and cell size on marine phytoplankton growth. Nature 390: 389-392.

Thompson JA, Marcelino LA, Polz MF (2002) Heteroduplexes in mixed-template amplifications: formation, consequence and elimination by 'reconditioning PCR'. Nucleic Acids Res 30: 20832088. .

Weber L, Volker C, Oschlies A, Burchard H (2007) Iron profiles and speciation of the upper water column at the Bermuda Atlantic Time-series Study site: a model based sensitivity study. Biogeosciences 4: 689-706.

Wells ML (1999) Manipulating Iron Availability in Nearshore Waters. Limnology and Oceanography 44: 1002-1008.

Wells ML, Price NM, Bruland KW (1994) Iron Limitation and the Cyanobacterium Synechococcus in Equatorial Pacific Waters. Limnology and Oceanography 39: 1481-1486.

Wu J, Boyle E, Sunda W, Wen L-S (2001) Soluble and Colloidal Iron in the Oligotrophic North Atlantic and North Pacific. Science 293: 847-849.

Wu J, Luther GW (1995) Complexation of Fe(III) by natural organic ligands in the Northwest Atlantic Ocean by a competitive ligand equilibration method and a kinetic approach. Marine Chemistry 50: 159-177.

Zinser ER, Lindell D, Johnson ZI, Futschik ME, Steglich C, Coleman M, Wright MA, Rector T, Steen R, McNulty NP, Thompson LR, Chisholm SW (2009) Choreography of the Transcriptome, Photophysiology, and Cell Cycle of a Minimal Photoautotroph, Prochlorococcus. PLOS ONE. 

CHAPTER FIVE

Summary and Future Directions 


\section{SUMMARY AND FUTURE DIRECTIONS}

The work presented here represents steps towards understanding the link between iron biogeochemical cycling and primary productivity in the open ocean. Inside a phytoplankton cell, these two biochemical cycles overlap and interact in a complex way. With its minimal genome, Prochlorococcus is an ideal system in which to study the intersection of these biogeochemical cycles, and because Prochlorococcus is globally abundant, these types of studies will be globally relevant. The theme that emerges from this work is that iron both distinguishes Prochlorococcus from other closely related organisms, like Synechococcus, and contributes to the genomic and physiological diversity among Prochlorococcus ecotypes.

\section{Summary}

Through comparing the growth of two Prochlorococcus strains over a range of iron concentrations we were first convinced that iron plays an important role in the diversity among the closely-related Prochlorococcus ecotypes (Chapter 2). Though MIT9313 is a larger cell, and has an elevated concentration of light-harvesting complexes for adaptation to low-light intensity, it survives at lower iron-concentrations than the small and high-lighted adapted MED4. We hypothesize that the adaptation of MED4 to the high iron-regime of the Mediterranean Sea encouraged the loss of high-affinity iron acquisition systems and allowed the maintenance of numerous iron-requiring proteins in the genome. Despite the contrast between MED4 and MIT9313 growth at low iron concentrations, in both strains, light intensity did not influence the concentration of iron that caused limitation of growth rate (Chapter 2). When we compared the concentration of Fe' that supports the growth of natural Prochlorococcus populations in the Equatorial Pacific to the concentration of Fe' that supports growth in the laboratory cultures, we were amazed to see that cultures are completely inhibited by iron limitation at concentrations of Fe' that would support maximal Prochlorococcus growth in the ocean (Chapter 2). Possibly, the iron physiologies of MED4 and MIT9313 are not comparable to the iron physiologies of native Equatorial Pacific Prochlorococcus. Or, after decades in high-iron culture medium, MED4 and MIT9313 have lost the capability to cope with extremely low iron concentrations. Or, specific aspects of iron speciation in the field, such as binding to organic ligands, increases the pool of iron that is available to Prochlorococcus, an effect that is not seen with the synthetic ironchelator, EDTA, in the culture medium. 
Intrigued by the ability of MIT9313 to survive at lower Fe' concentrations than MED4, and the apparent disconnect between iron availability in the open-ocean and in culture, we assessed the whole-genome expression of MED4 and MIT9313 in response to changing iron availability in an effort to understand the molecular basis for iron metabolism in Prochlorococcus (Chapter 3). We expected iron metabolism to be firmly encoded in the core genome because of its importance in photosynthetic electron transfer. Yet, we found that a substantial portion of the iron-responsive genes in MED4 and MIT9313 belong to the non-core genomes of each strain. Though many Prochlorococcus core genes were iron-responsive in each strain, only three were differentially expressed in both MED4 and MIT9313 during iron stress and recovery from iron stress (Chapter 3). These genes encode ferredoxin, an electron transfer protein associated with photosystem I (PSI), flavodoxin, the well-characterized iron-free replacement of ferredoxin, and afuA/idiA, a homolog to the iron-deficiency induced protein that may be the periplasmic Febinding component of an iron $\mathrm{ABC}$-transport system. Notably, we found that the afuA/idiA region of both MED4 and MIT9313 and all other sequenced Prochlorococcus genomes is particularly active during changes in iron availability. Additionally, MED4 is lacking several genes in this region that are present in MIT9313 and expressed during iron deprivation including a porin, peptidase, and uncharacterized iron-regulated protein. We hypothesize that the afuA/idiA region may be influential, at least in part, in determining the iron physiology of Prochlorococcus ecotypes and the superior ability of MIT9313 to grow at low iron concentrations.

Finally, we turned to examine Prochlorococcus and Synechococcus iron physiology in the natural environment (Chapter 4). We discovered that in the same sample of water, Synechococcus responds to iron addition but Prochlorococcus does not. This evidence is intriguing given that these two groups of cells co-exist, and presumably are adapted to the same chemical and physical conditions. We also describe a plan to develop a molecular indicator of iron stress for field populations of Prochlorococcus. We have seen that isiB is one of few Prochlorococcus core genes that responded quickly and strongly to iron availability and it is common in environmental metagenomic databases so we can assess the diversity of isiB sequences in field populations. Lastly, we describe the steps that remain to be completed before $i s i B$ transcription can be measured in natural populations of Prochlorococcus and Synechococcus (Chapter 4). 


\section{Future directions}

This work inspires many more questions than it answers about the influence of iron on Prochlorococcus ecology and the influence of Prochlorococcus on iron biogeochemical cycles. One major outstanding question pertains to the relevance of our culture-based studies to natural populations of Prochlorococcus in the open-ocean environment. We are particularly intrigued by the possibility that total iron concentrations (as opposed to Fe' concentrations) are relevant to Prochlorococcus in the field (Chapter 2). We propose that by measuring iron-stress in Prochlorococcus over gradients in iron concentration and speciation that it will be possible to determine which forms of iron best support Prochlorococcus growth and photosynthetic efficiency. In Chapter 4, we describe in detail the future work necessary to make these types of measurements. Additionally, by testing the iron-requirement of a strain isolated from the Pacific Ocean, for example MIT9515, we could test the possibility that MED4 and MIT9313 are simply not adapted to low-iron regimes. We also wonder how the iron-responsive genes we have identified in MED4 and MIT9313 are distributed in the open ocean. Probing the Prochlorococcus-rich environmental metagenomic databases will inform questions about the biogeography of iron-responsive genes and the importance of iron in different areas of the open ocean. For example, will we find more MED4 iron-responsive genes in the high-light environments or in high-iron regimes like the Mediterranean?

Despite the differential expression of hundreds of genes in MED4 and MIT9313 during iron starvation, we are still left with an incomplete picture of how Prochlorococcus uses, stores, and acquires iron to meet its metabolic requirements. Future work will dig deeper into how genes are transcribed in response to iron availability by imposing more severe conditions of iron stress on the cells, such as steady-state iron limitation. Also, we are confident that an examination of the differential translation of the MED4 and MIT9313 genomes in response to iron will be revealing in terms of the function, location, and structure of important iron-metabolism proteins. Also, most of the iron-responsive genes we identified are of unknown function and may be key to understanding the numerous and complex dimensions in the environment that influence Prochlorococcus ecology. By systematically characterizing the numerous proteins of unknown function in the Prochlorococcus core and non-core genomes there is much to be learned about microbial diversity and the characteristics that distinguish Prochlorococcus from other primary producers in the marine environment. As no reliable genetic system exists for Prochlorococcus at 
the time of this writing, characterization of unknown proteins may be most feasible through structural analysis.

Finally, we hope future work will examine how Prochlorococcus iron metabolism intersects with other aspects of cellular metabolism. We have begun these types of analysis by asking how iron requirement varies with acclimation to different light intensities and how wholegenome expression in response to iron starvation compares to the response of Prochlorococcus to other types of stress. We are now poised to consider how the different metabolisms and physiologies of Prochlorococcus interact and overlap in a system-wide view of the Prochlorococcus cell. By considering the entire cell-system we will better understand how the availability of inorganic nutrients and global patterns of primary productivity are linked through the activity of Prochlorococcus. 



\begin{abstract}
APPENDiX A
Use of stable isotope-labelled cells to identify active grazers of picocyanobacteria in ocean surface waters

Jorge Frias-Lopez, Anne W. Thompson, Jacob Waldbauer, and Sallie W. Chisholm
\end{abstract}

Reprinted with permission from Environmental Microbiology

(C) 2009 Society for Applied Microbiology and Blackwell Publishing Ltd

Frias-Lopez, J., Thompson, A., Waldbauer, J., Chisholm, S.W. (2009). Use of stable isotopelabelled cells to identify active grazers of picocyanobacteria in ocean surface waters.

Environmental Microbiology 11(2): 512-525. 


\section{Use of stable isotope-labelled cells to identify active grazers of picocyanobacteria in ocean surface waters}

OnlineOpen: This article is available free online at www.blackwell-synergy.com

Jorge Frias-Lopez, ${ }^{1 *}$ Anne Thompson,,${ }^{1,2}$ Jacob Waldbauer ${ }^{1,3}$ and Sallie W. Chisholm 1

${ }^{1}$ Department of Civil and Environmental Engineering,

Massachusetts Institute of Technology, 15 Vassar

Street, Cambridge, MA 02139, USA.

${ }^{2}$ MIT/Woods Hole Joint Program in Biological

Oceanography, Cambridge, MA 02139, USA.

${ }^{3}$ MITWoods Hole Joint Program in Chemical

Oceanography, Cambridge, MA 02139, USA.

\section{Summary}

Prochlorococcus and Synechococcus are the two most abundant marine cyanobacteria. They represent a significant fraction of the total primary production of the world oceans and comprise a major fraction of the prey biomass available to phagotrophic protists. Despite relatively rapid growth rates, picocyanobacterial cell densities in open-ocean surface waters remain fairly constant, implying steady mortality due to viral infection and consumption by predators. There have been several studies on grazing by specific protists on Prochlorococcus and Synechococcus in culture, and of cell loss rates due to overall grazing in the field. However, the specific sources of mortality of these primary producers in the wild remain unknown. Here, we use a modification of the RNA stable isotope probing technique (RNA-SIP), which involves adding labelled cells to natural seawater, to identify active predators that are specifically consuming Prochlorococcus and Synechococcus in the surface waters of the Pacific Ocean. Four major groups were identified as having their 18S rRNA highly labelled: Prymnesiophyceae (Haptophyta), Dictyochophyceae (Stramenopiles), Bolidomonas (Stramenopiles) and Dinoflagellata (Alveolata). For the first three of these, the closest relative of the sequences identified was a photosynthetic organism, indicating the presence of mixotrophs among picocyanobacterial predators. We conclude that the use of RNA-SIP is

Received 7 May, 2008; accepted 13 September, 2008. ${ }^{*}$ For correspondence. E-mail jfrias@mit.edu; Tel. (+1) 617253 1771; Fax (+1) 6173240336 .

Re-use of this article is permitted in accordance with the Creative Commons Deed, Attribution 2.5, which does not permit commercial exploitation. a useful method to identity specific predators for picocyanobacteria in situ, and that the method could possibly be used to identify other bacterial predators important in the microbial food-web.

\section{Introduction}

The mechanisms that regulate microbial communities are a central issue in ocean ecology. Phagotrophic protists and viruses are the main sources of mortality for these microbes in oligotrophic environments (Fuhrman and Campbell, 1998; Partensky et al., 1999a) and play an important role in shaping microbial communities in the ocean (so-called 'top-down' regulation) (Sherr and Sherr, 2002; Pernthaler, 2005). One of the outstanding questions is precisely how the food-web is structured: which protists eat which microbes?

Grazing activity by eukaryotes is a major factor of bacterial mortality in the ocean and a major force for shaping microbial communities in those environments (Jurgens and Matz, 2002). Heterotrophic nanoflagellates and ciliates are considered to be the primary grazers on planktonic marine bacteria (Sherr et al., 1989; Simek and Chrzanowski, 1992; Cho et al., 2000; Sherr and Sherr, 2002). In general, grazing by bacterivorous protists upon suspended bacteria is size selective (Chrzanowski and Símek, 1990; Gonzalez et al., 1990; Simek and Chrzanowski, 1992; Jürgens and Güde, 1994; Anderson and Rivkin, 2001) with most protists grazing preferentially on medium-sized bacterial cells.

Because Prochlorococcus and Synechococcus numerically dominate the oxygenic phototrophs in ocean waters (Chisholm et al., 1988; Partensky et al., 1999a,b), understanding their sources of mortality is central to understanding the structure of the microbial food-web, and the regulation of marine productivity and nutrient cycling in the ocean. Laboratory studies using cultured heterotrophic flagellates and ciliates have shown that they can survive when fed Prochlorococcus and Synechococcus (Christaki et al., 1999; Guillou et al., 2001) and that some feed preferentially on one or the other (Christaki et al., 1999; Guillou et al., 2001). Studies using natural nanoflagellate populations show that the nanoflagellate community composition shapes the picoautotrophic community structure and, vice versa, the picoautotrophic 
community structure favours or inhibits the growth of some nanoflagellates groups (Christaki et al., 2005). However, these studies do not address the question of the identity of the grazers feeding on bacteria.

While rates of grazing-induced mortality of picocyanobacteria have been measured in situ (Sherr et al., 1987; Hall et al., 1993; Ishii et al., 2002; Massana et al., 2002; Worden and Binder, 2003; An-Yi et al., 2007), the specific identity of the grazers feeding on these cells has not been studied. In the present work, we have used a modification of a RNA stable isotope probing technique (RNA-SIP) (Radajewski et al., 2000; Manefield et al., 2002; Lueders et al., 2004) to identify eukaryotic cells that consume Prochlorococcus and Synechococcus in surface waters at the Hawaii Ocean Time Series (HOT) station ALOHA. A similar approach had been previously used to identify micropredators of Escherichia coli in a sample of agricultural soil (Lueders et al., 2006). The use of this method avoids problems associated with using non-active bacteria (González et al., 1990; Landry et al., 1991; del Giorgio et al., 1996; Ishii et al., 2002; Koton-Czaarnecka and Chrost, 2003), and enables molecular taxonomic resolution.

\section{Results and discussion}

Characterization of the indigenous eukaryotic protist community

We first characterized the diversity of protists in our sample, collected from the study site, Station ALOHA (Hawaii Ocean Time Series) through the analysis of the indigenous 18S rDNA sequences (Figs $1 \mathrm{~A}$ and 2 and Fig. S1). The community was similar to those reported for other oligotrophic surface ocean waters (Countway et al., 2005; 2007; Not et al., 2007), in terms of firstand second-rank marine protistan and Super-group taxa defined by Adl and colleagues (2005). Alveolates, and specifically Dinozoa, including novel Alveolate groups I and II (NAI and NAII), are among the most abundant sequences found. Stramenopiles, including novel Marine Stramenopiles (MAST), are also well represented (Figs $1 \mathrm{~A}$ and 2 and Fig. S1).

\section{Incubation experiments with labelled cultures}

To determine which protists from this community most actively grazed on Prochlorococcus and Synechococcus, ${ }^{13} \mathrm{C}$ - and ${ }^{15} \mathrm{~N}$-labelled cultures of these cyanobacteria were added to seawater samples and incubated for 1 day, allowing the indigenous community to consume the labelled cells (see Experimental procedures for details). After $24 \mathrm{~h}$, the microbial community was collected by filtration, RNA was extracted, and 'heavy' (labelled) and 'light' (unlabelled) RNA was separated by density gradient ultracentrifugation. Density-resolved $18 \mathrm{~S}$ rRNA sequences were amplified, sequenced and analysed. Sequences from the labelled subfraction (which are enriched in a subset of sequences as they are physically separated from the bulk community before sequencing) are interpreted as being derived from eukaryotic cells that consumed high numbers of labelled Prochlorococcus or Synechococcus cells during the incubation. Sequences in the unlabelled RNA fraction represent protists that did not graze on the labelled cells during the incubation. Because different levels of RNA labelling are likely to occur depending on what fraction of the diet of a particular grazer consists of Prochlorococcus and Synechococcus, we analysed only the most highly labelled fractions (Fig. 3).

We recognize that there are, theoretically, a number of possible indirect routes for the heavy isotopes to end up in the $18 \mathrm{~S}$ rRNA. We analysed these possibilities in detail in a separate section below, and conclude that direct grazing on Prochlorococcus and Synechococcus is the most consistent explanation for the incorporation of label into $18 \mathrm{~S}$ rRNA in our experiments.

\section{Community structure analysis using terminal restriction} fragment length polymorphism ( $T$-RFLP)

Before analysing the sequences of rRNA from the labelled and unlabelled fractions in detail, we assessed the quality of the biological replicates and general differences and similarities among the treatments, using terminal restriction length polymorphism (T-RFLP) and cluster analysis (GEPAS, http://www.gepas.org) (Dollhopf et al., 2001). The eukaryotic cells at the onset of the experiment (time 0), as well as those that remained unlabelled after a $24 \mathrm{~h}$ incubation (i.e. those that did not prey on either Prochlorococcus or Synechococcus), cluster together in both replicates (Fig. 4). The similarity of these two groups indicates that there were no significant changes in the food-web structure in the incubation bottles during the $24 \mathrm{~h}$ incubation. More importantly, the $18 \mathrm{~S}$ rRNA sequences containing the Prochlorococcusderived label and Synechococcus-derived label clustered separately from the time 0 and unlabelled rRNA samples, indicating that we are identifying a specific subset of the community that is preying upon these cyanobacteria. Furthermore, the predator sequences originating from addition of Prochlorococcus and Synechococcus did not cluster together, suggesting distinct predators for these two types of cyanobacteria, consistent with observations from laboratory studies (Guillou et al., 2001; Pernthaler, 2005). 


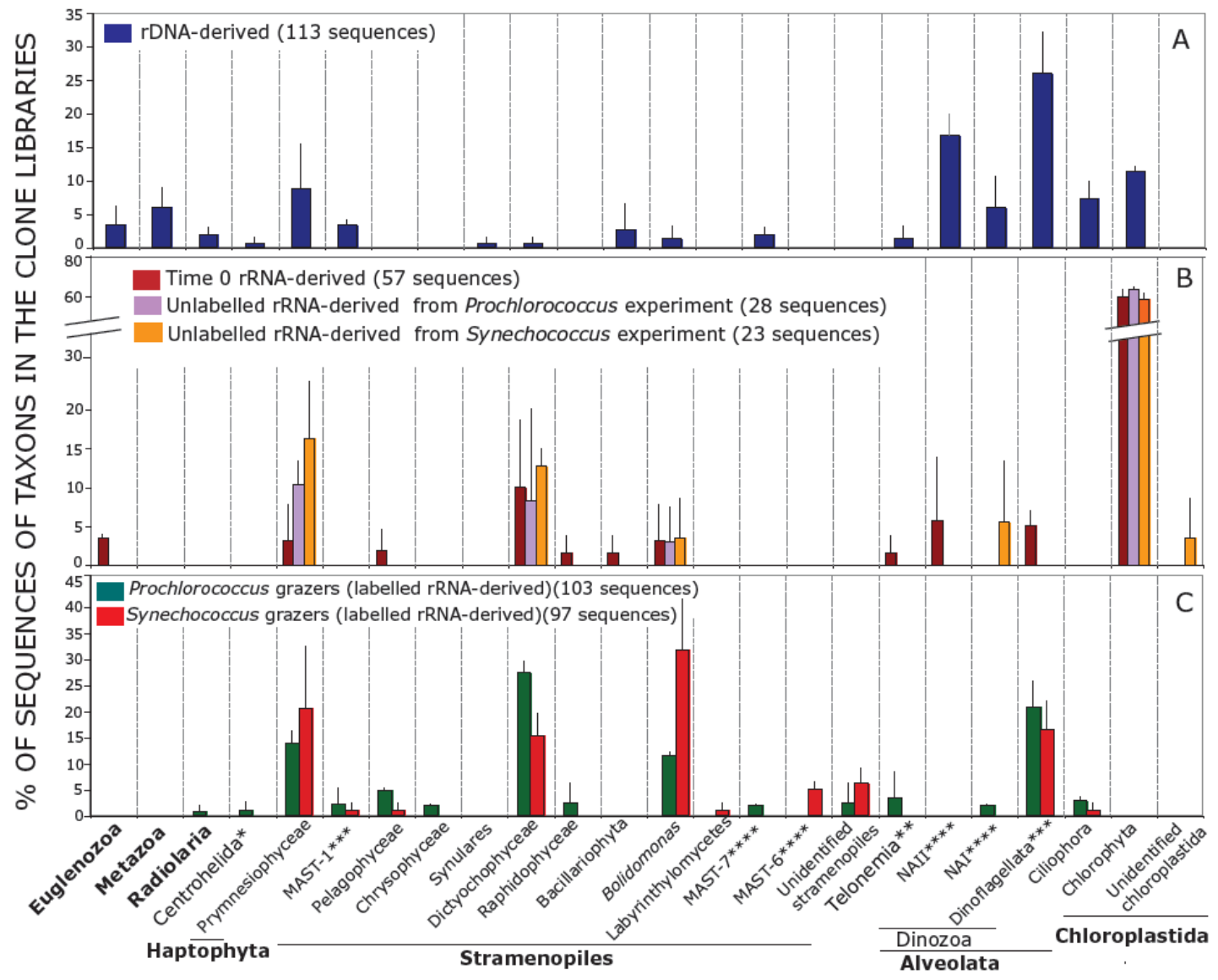

Fig. 1. Phylogenetic assignments and relative frequencies of the rDNA sequences from indigenous eukaryotic community, and the labelled and unlabelled rRNA fractions in the experimental treatments.

A. rDNA extracted from the total community.

B. Unlabelled fractions from the density gradient separations and time 0 samples.

C. Samples with label originating from Prochlorococcus or Synechococcus added to the experimental bottles.

Error bars represent the standard deviation of the values obtained for the biological duplicates of the libraries. Phylogenetic assignment follows Adl and colleagues (2005) with classification at the first- (in bold) and second-rank taxonomic level except when indicated as follows: "Super-groups, "**Phylum, "**third-rank taxonomic level and ${ }^{* * * *}$ novel Alveolate groups I and II (NAI and NAII), or the novel MAST following Not and colleagues (2007).

Fig. 2. Unrooted phylogenetic tree inferred by maximum likelihood (ML) analysis of the reference sequences used in the phylogenetic analysis of the clone libraries presented in this work (see Supporting information). Selected representative clones and colour circles indicate the phylogenetic adscription of the sequences obtained in the different clone libraries. Blue clones and circles: sequences originating from the DNA-derived libraries. Purple clones and circles: sequences originating in the unlabelled fractions from the density gradient separations and time 0 samples. Green clones and circles: sequences originating from the labelled fraction of the Prochlorococcus inoculation experiment. Red clones and circles: sequences originating from the labelled fraction from the Synechococcus inoculation experiment. Partial sequences ranging from a minimum of $604 \mathrm{bp}$ up to $827 \mathrm{bp}$ were used in the alignment. Bootstrap values over $50 \%$ are indicated on the internal branches obtained from Bootstrap values $<50 \%$, which have been omitted. The proportion of invariant sites $(I)$ was 0.214 . The scale bar indicates $5 \%$ divergence. Classification is based on Adl and colleagues (2005) and Not and colleagues (2007). All groups correspond to first and second rank according to Adl and colleagues (2005) except *Super-group and ${ }^{* *}$ Phylum (Shalchian-Tabrizi et al., 2006), ${ }^{* * *}$ third-rank taxonomic level and ${ }^{* * * *}$ novel Alveolate groups I and II (NAI and NAII), or the novel MAST following Not and colleagues (2007). 


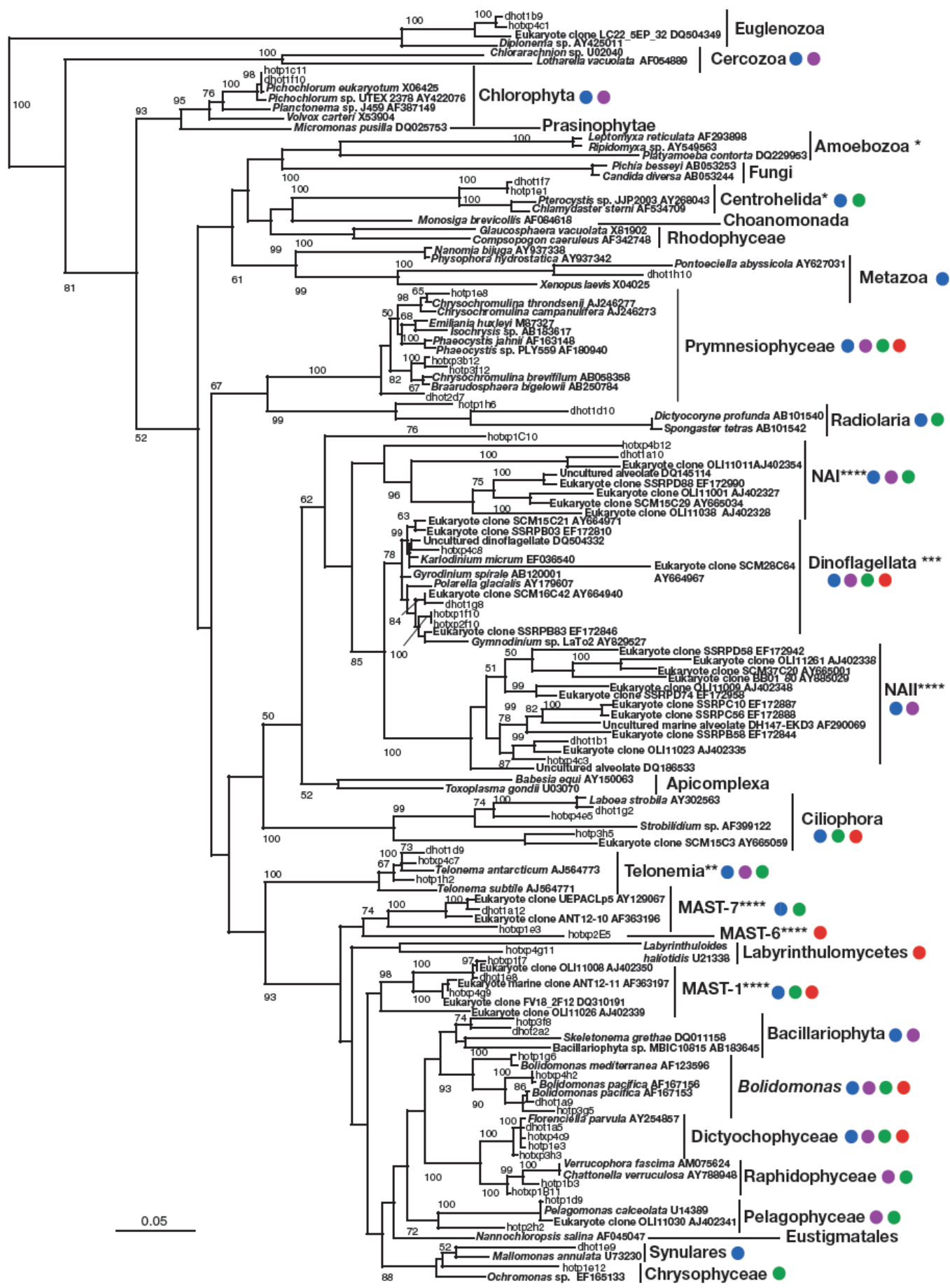

(C) 2008 The Authors

Journal compilation @ 2008 Society for Applied Microbiology and Blackwell Publishing Ltd, Environmental Microbiology, 11, 512-525 
O Unlabelled fractions used in the experiments

O Labelled fractions used in the experiments

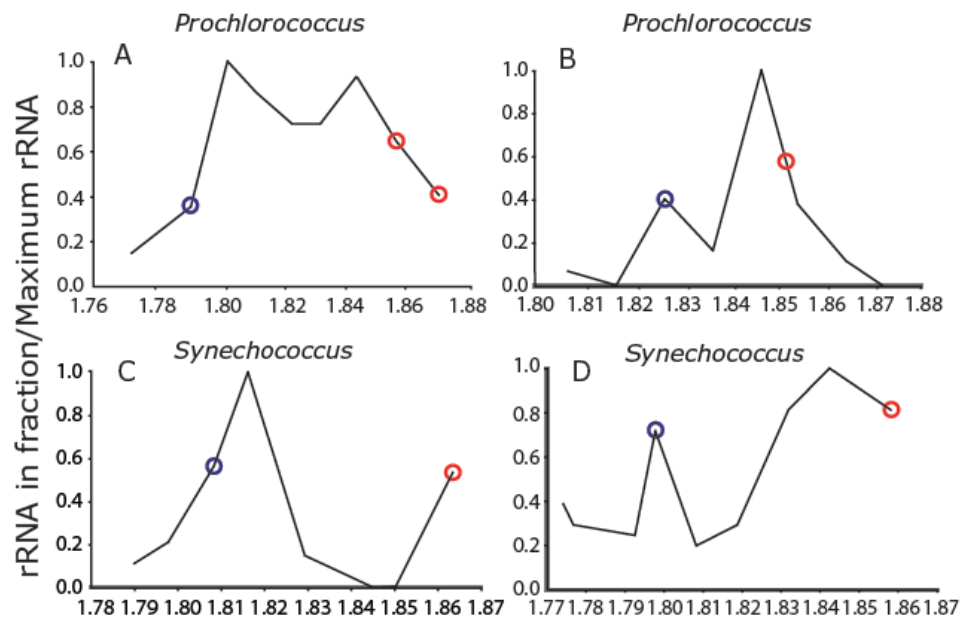

CsTFA buoyant density $\left(\mathrm{g} \mathrm{ml}^{-1}\right)$

Fig. 3. Relative amount of rRNA in different fractions separated by density gradient centrifugation of $18 \mathrm{~S}$ rRNA analysed in this study. Two peaks of RNA were detected in each sample, the lighter containing sequences that did not incorporate the isotopic label $\left({ }^{15} \mathrm{~N}\right.$ and ${ }^{13} \mathrm{C}$ ) during the $24 \mathrm{~h}$ incubation, and the heavier to the RNA greatly enriched in the heavy isotope, i.e. from cells that incorporated label from the Prochlorococcus and Synechococcus that were added to the samples. The particular samples indicated in a blue circle were analysed as the 'unlabelled fraction' from the experimental bottles, and those in a red circle the 'heavily labelled' fraction. These particular samples were

chosen to maximize the sample size while at the same time avoiding cross-contamination of light and heavy RNA in the subsequent analyses. (A) and (B) represent sequences from biological replicates of samples amended with labelled Prochlorococcus (in A two heavy fractions were used to increase the amount of total RNA used for constructing the clone libraries) and (C) and (D) those amended with Synechococcus. Total RNA was detected fluorometrically using Ribogreen (see Experimental procedures).

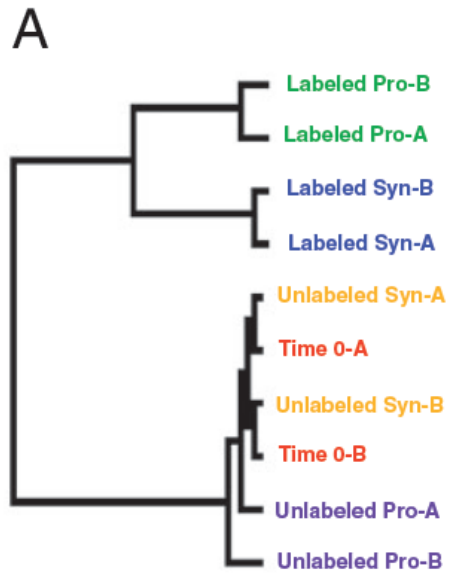

Hhal digestion

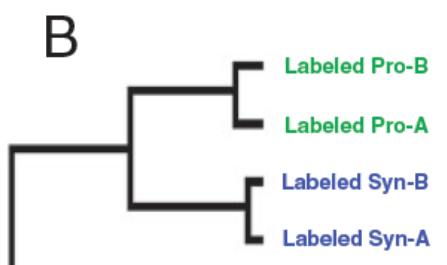

Fig. 4. Self-organizing tree (SOTA) of terminal restriction fragment length

polymorphism (T-RFLP) profiles emerging from the 18S rRNA sequences from the different experimental treatments. Samples digested (A) with Hhal and (B) with Rsal.

Time 0: T-RFLP profile of rRNA from the entire eukaryotic community at the beginning of the experiment. Unlabelled Pro and Unlabelled Syn: T-RFLP profiles of the unlabelled eukaryotic rRNA, collected from the density gradient, from the bottles that were incubated with labelled Prochlorococcus $\begin{array}{ll}\text { Time 0-A } & \text { were incubated with labelled Prochlorococcus } \\ & \text { and Synechococcus respectively. Labelled } \\ & \text { Pro and Labelled Syn: T-RFLP profiles of th }\end{array}$ $\begin{array}{ll}\text { Time 0-A } & \text { were incubated with labelled Prochlorococcus } \\ & \text { and Synechococcus respectively. Labelled } \\ & \text { Pro and Labelled Syn: T-RFLP profiles of the }\end{array}$ Unlabeled Syn-B heavily labelled eukaryotic rRNA that was collected from the same gradient. A and B next to the data points represent the two biological replicates.

\section{Unlabeled Pro-A}

Unlabeled Pro-B

Rsal digestion 
Analysis of the unlabelled and labelled 185 rRNA sequences

We next analysed the identity of the unlabelled and labelled eukaryotes by cloning and sequencing the $18 \mathrm{~S}$ rRNA fragments from the heavily labelled and unlabelled fractions isolated from the density gradient separation (Fig. 3). Heavily labelled fractions represent eukaryotes that have eaten either Prochlorococcus or Synechococcus. The unlabelled fractions represent eukaryotes in the community with relatively high levels of rRNA that did not assimilate label from the cyanobacteria. As has been reported previously (Stoeck et al., 2007) the sequences in the unlabelled rRNA-derived library are substantially different from those in the rDNA library (Figs $1 \mathrm{~A}$ and $\mathrm{B}$ and 2; Figs S1 and S2), showing that there are some members of the community that are much more 'active' (as measured by rRNA levels) than others.

Most sequences obtained in the rRNA-derived library from the time 0 samples and unlabelled fractions represented members of the Chlorophyta, principally close relatives of the genus Picochlorum (Figs 1B and 2 and Fig. S2). Other taxonomic groups identified in these libraries included the Dictyochophyceae (Stramenopiles) and Prymnesiophyceae (Haptophyta), and in smaller numbers relatives of members of Raphidophyceae, Bolidomonas, Bacillariophyta, Pelagophyceae (all Stramenopiles), Euglenozoa and Dinozoa (Alveolota) (Figs 1B and 2 and Fig. S2).

The sequences that appeared in the labelled fractions (Figs $1 \mathrm{C}$ and 2, Figs S3 and S4) - i.e. from cells grazing on Prochlorococcus and Synechococcus - belonged primarily to four groups: the Prymnesiophyceae, Dictyochophyceae, Bolidomonas and Dinoflagellata. Dictyochophyceae dominated the 18S rRNA sequences that had incorporated label from Prochlorococcus, while Bolidomonas dominated those that had incorporated the label from Synechococcus (Fig. 1C), but it appears that the four dominant grazers consume both types of cells. Novel MAST also appeared in both labelled rRNA-derived libraries and they have been identified as non-pigmented heterotrophic flagellates with bacterivory activity (Massana et al., 2002). Some taxonomic groups appear to be specific to either Prochlorococcus or Synechococcus (Figs $1 \mathrm{C}$ and 2, Figs S3 and S4), but this could be simply due to the small library sample size. Certain groups that were present in the labelled rRNA-derived clone libraries but were absent in the unlabelled rRNA-derived clone libraries could have been simply masked by the high dominance of Chlorophyta in the rRNA-derived clone libraries in relation to the rest of identified phylogenetic groups.

Ciliates, which are considered important grazers in some aquatic environments (Sherr and Sherr, 2002; Pem- thaler, 2005), represent a small fraction of the labelled sequences, which is consistent with recent work showing that subtropical marine ciliates exhibit almost no grazing activity on bacterium-sized particles (An-Yi et al., 2007) and with the experimental results by Christaki and colleagues (1999) showing that Prochlorococcus and Synechococcus proved to be poor food sources for ciliate growth.

The most striking observation in these results is that three of the four most abundant sequences in the labelled 18S rRNA fraction belong to the taxa Prymnesiophyceae, Dictyochophyceae and Bolidomonas, whose characterized members are photosynthetic. Two groups present in the labelled 18S rRNA fraction, the Pelagophyceae and Bolidomonas, have not previously been found to consume bacteria. Some Pelagophyceae feed heterotrophically on dissolved organic matter (Lomas et al., 2001) but this group has previously been described as nonphagotrophic (Cavalier-Smith and Chao, 2006). Characterized members of the Bolidomonas, the most frequently detected labelled group in the Synechococcus-fed samples (Figs $1 \mathrm{C}$ and 2 and Fig. S4), are all photosynthetic. While some members of the Dictyochophyceae, which dominate the clone libraries from the labelled Prochlorococcus-fed samples (Figs 1C and 2 and Fig. S3), are heterotrophs, the closest relative to the sequences we have identified is Florenciella parvula, which is photosynthetic. Also among the identified predators of Prochlorococcus and Synechococcus are representatives of groups known to be capable of mixotrophy, including the Chrysophyceae (Nygaard and Tobiesen, 1993), Prymnesiophyceae (Nygaard and Tobiesen, 1993; Hansen and Hjorth, 2002) and Dinoflagellata (Hansen and Nielsen, 1997). Almost all of the sequences from the labelled clone libraries belong to plastid-containing lineages; only sequences identified as relatives of Telonema (phylum Telonemia) (Shalchian-Tabrizi et al., 2006) and Centrohelida come from groups not known to contain autotrophic members.

Previous work had already presented evidence that mixotrophic nanoflagellates are important predators in surface waters and may make up more than $50 \%$ of the bacterivory in them, and that they are more abundant near ocean surface waters than in the deeper euphotic zone (Arenovski et al., 1995; Caron, 2000). Moreover, previous studies have demonstrated that pigmented and nonpigmented nanoflagellates had similar grazing rates on heterotrophic bacteria (Hall et al., 1993).

\section{Detection of label in plastid 16S rRNA}

To further test the mixotrophy hypothesis we examined whether the labelled fraction contained plastid DNA using primers designed specifically for the 16S rRNA sequence 


\section{J. Frias-Lopez, A. Thompson, J. Waldbauer and S. W. Chisholm}

in chloroplast DNA (Table S1). We designed these primers specifically to amplify plastid 16S rRNA genes, but not Prochlorococcus and Synechococcus 16S rRNA, since the latter would have dominated our signal. This meant we did not recover as many plastid sequences as we might have if we had used published plastid 16S rRNA primers (Fuller et al., 2006), but this was an unavoidable limitation, given the experimental design.

Two of the primer sets for plastid 16S rRNA (primers sets 6 and 15, Table S1) yielded PCR products of the expected size, and in the case of primer set 15 the product was long enough (approximately $650 \mathrm{bp}$ ) to be sequenced and analysed. Although it is difficult to determine the exact affiliation of these chloroplast sequences given the short length of the amplified PCR product, and the limited coverage of chloroplast sequences from different plastid-containing phylogenetic groups in the database, the phylogenetic analysis showed that the amplified sequences from the labelled fraction were indeed from chloroplasts (Fig. S5). Furthermore, the phylogenetic analysis showed that the closest relatives of the chloroplasts identified in our labelled fraction were related to Bolidomonas mediterranea and diatom chloroplasts (Fig. S5). As there are a limited number of chloroplast sequences representing other groups of Stramenopiles in the databases, and given the short size of the analysed product, the exact phylogenetic affiliation of these sequences is not entirely clear. The key finding, however, is that all of the sequences obtained cluster with chloroplasts indicating that the heavy label ended up in eukaryotic cells capable of photosynthesis.

\section{Analysis of alternative routes for label incorporation}

In this and other types of labelling experiments with natural populations, the possibility that the isotopic label might have been acquired by protists via a route other than phagotrophic predation must be considered. For example, it is conceivable that the label might have passed through a dissolved phase, either organic or inorganic, and was acquired through non-phagotrophic nutrient uptake. Alternatively, the label could have been initially acquired by bacterial heterotrophs that were subsequently grazed by phagotrophs. Below we consider each of these possibilities in turn, and present evidence that they do not appear to be playing a role in these experiments.

The labelled cyanobacterial biomass could have been transformed to dissolved inorganic carbon (DIC) through respiration, either by the picocyanobacteria themselves or by other heterotrophs. Had a substantial amount of the added biomass been respired, that labelled carbon would have become broadly available for fixation by all of the autotrophs in the sample, which would then appear in the labelled fraction. In fact, the most abundant sequences in the unlabelled rRNA-derived clone libraries - the photoautotrophic Chlorophyta - were not represented in the labelled fraction (Figs $1 \mathrm{~B}$ and $\mathrm{C}$ and 2, Figs S2-S4). This demonstrates that no significant quantity of labelled DIC was available for photosynthetic fixation, and passage of the label through the dissolved carbonate pool can be excluded.

Another possibility would be that the initially supplied, isotopically labelled biomass might have entered the dissolved organic carbon (DOC) pool by exudation, lysis or 'sloppy feeding' by zooplankton. The latter two mechanisms would result in substantial declines in the picocyanobacterial population during the experiment; however, the concentration of picocyanobacteria did not change dramatically over the $24 \mathrm{~h}$ of incubation. In all cases the initial and final concentration, after $24 \mathrm{~h}$ of incubation, of both Prochlorococcus and Synechococcus was of $10^{5}$ cells $\mathrm{ml}^{-1}$, suggesting that mechanisms involving cell death (including lysis and sloppy feeding) did not release large amounts of biomass into the dissolved phase. To consider exudation, we can use the Prochlorococcus addition experiment as an example. Prochlorococcus MED4 cells were added to the seawater sample at a concentration of $1.7 \times 10^{5}$ per $\mathrm{ml}$ and typically contain about $60 \mathrm{fg}$ of carbon per cell (Bertilsson et al., 2003). If we imagine that the added Prochlorococcus could somehow exude all of their initial labelled carbon as DOC - while suffering no great decline in cell numbers - this is equivalent to the addition of $0.9 \mu \mathrm{M}$ of ${ }^{13} \mathrm{C}$-DOC, clearly an upper limit for the potential contribution of the isotopically labelled Prochlorococcus to the DOC pool. Typical surface total DOC concentrations at station ALOHA, where the samples for this study were taken, are around $75 \mu \mathrm{M}$, of which $40 \mu \mathrm{M}$ is likely refractory organic matter that turns over very slowly (Carlson, 2002). Hence there is roughly $35 \mu \mathrm{M}$ of labile DOC available for rapid heterotrophic consumption. Addition of Prochlorococcusderived ${ }^{13} \mathrm{C}$-DOC to this could result in a $36 \mu \mathrm{M}$ pool of labile DOC with maximum ${ }^{13} \mathrm{C}$ content of 3.5 atom\%, which is in tum the upper limit for labelling by DOC consumption. Similar considerations limit the ${ }^{13} \mathrm{C}$ content of DOC in the Synechococcus addition experiments to 10.6 atom\%.

Next, we consider the extent of labelling of the heavy RNA fractions in our incubation experiments. The difference in buoyant density between heavy and light RNA fractions in these experiments ranged from 0.034 to $0.078 \mathrm{~g} \mathrm{ml}^{-1}$ (Fig. 3), equal to or exceeding the buoyancy differences $\left(0.035-0.04 \mathrm{~g} \mathrm{ml}^{-1}\right)$ observed by Lueders and colleagues (2004) for $100 \%{ }^{13} \mathrm{C}$-labelled SSU rRNA. This large difference in buoyant density suggests that the heavy fractions analysed in this experiment were highly labelled, likely in excess of 90 atom $\%{ }^{13} \mathrm{C}$. This is far 
greater than the $3-11 \%$ possible from DOC consumption, even under the assumption of maximally rapid exudation by the added cyanobacteria. The buoyancy differences observed here in excess of the $\sim 0.4 \mathrm{~g} \mathrm{ml}^{-1}$ reported by Lueders and colleagues (2004) may reflect ${ }^{15} \mathrm{~N}$ incorporation and/or differences in centrifugation conditions. In any event, the heavy RNA in these experiments is much too highly labelled to derive from heterotrophic consumption of DOC.

A third, even more mechanistically complicated possibility is the direct and specific consumption of picocyanobacteria by heterotrophic bacteria or the consumption of labelled DOC exuded by, or otherwise released from, the picocyanobacteria by those heterotrophs. Protistan predators can then graze on these labelled heterotrophs. If this occurred, the $18 \mathrm{~S}$ sequences observed in the heavy fraction would reflect grazing activity, though not specifically on Prochlorococcus or Synechococcus. Under this scenario, a subset of heterotrophic bacteria would become highly labelled, and their RNA should be found in the heavy fraction. To address this possibility, we constructed 16S rRNA clone libraries as described in Experimental procedures. If there had been transfer of labelled organic matter through heterotrophic bacteria at the level needed to fractionate differentially in a CsTFA gradient we would expect to find 16S rRNA sequences from heterotrophic bacteria. Forty-three clones from the labelled fractions were sequenced. Seventeen clones came from the fraction obtained from the bottles inoculated with labelled Prochlorococcus MED4 and in all cases the best BLASTN match for those sequences corresponded to Prochlorococcus marinus. Similarly, 26 clones coming from the fraction obtained from the bottles inoculated with labelled Synechococcus WH8102 and in all cases the best BLASTN match corresponded to Synechococcus. Additionally, 11 clones coming from the unlabelled fraction from the Prochlorococcus experiment were sequenced and $18 \%$ of those corresponded to $P$. marinus, while the rest were sequences from heterotrophic bacteria. These results demonstrate that 16S rRNA compositions of the labelled and unlabelled fractions were indeed distinct, and that heterotrophic bacteria did not appear to become highly labelled over the course of the incubation. We thus conclude that the labelled eukaryotes did not obtain their label indirectly via predation of heterotrophic bacteria.

\section{Conclusions and implications}

The reproducibility and intemal consistency of the results obtained in the study indicate that the use of RNA-SIP for studying the marine microbial food-webs in situ has tremendous potential. There are a multitude of variations on this experimental design that could yield many insights into the specific pathways of the flow of carbon and energy in the marine food-web. These particular results also reveal that a significant fraction of the eukaryotes that we identified as grazing specifically on Prochlorococcus and Synechococcus were likely mixotrophs - i.e. cells that utilize both phototrophy and phagotrophic heterotrophy as a way of obtaining nutrients and energy (Raven, 1997; Jones, 2000). While a few studies have provided evidence of the importance of mixotrophy in marine aquatic environments (Arenovski et al., 1995; An-Yi et al., 2007; Unrein et al., 2007), this is the first study to identify marine mixotrophs through their grazing activity on specific prey.

The adoption of mixotrophy as a survival strategy under oligotrophic oceanic conditions might confer a fitness advantage for a number of reasons (Raven, 1997). First, phagotrophy may be a way for relatively large eukaryotic cells to acquire inorganic nutrients such as $\mathrm{N}, \mathrm{P}$ and $\mathrm{Fe}$ in oligotrophic waters. Arenovski and colleagues (1995) presented experimental evidence of a decrease in the abundance of mixotrophic phototrophs under nutrient enrichment conditions, suggesting that phagotrophy is used under low dissolved nutrient concentrations, conditions that are normal in surface oligotrophic water. With their larger surface to volume ratio, picocyanobacteria like Prochlorococcus and Synechococcus likely have an advantage over larger eukaryotic cells in acquiring dissolved nutrients. Consuming cyanobacteria may also be a way for the larger cells to increase their relative fitness by reducing the abundance of their competitors for nutrients. Mixotrophy has been linked to survival of nanoflagellates under nutrient limitation (Unrein et al., 2007) and it has been shown that algal flagellates increase bacterivory under phosphate limitation (Nygaard and Tobiesen, 1993). Moreover, the metabolic costs of adding phagotrophic machinery to an otherwise photosynthetic metabolism may be rather low in comparison with the potential benefits (Raven, 1997).

Predation by mixotrophs also has implications for our understanding of the population dynamics of marine picocyanobacteria. While picocyanobacteria are generally the numerically dominant phytoplankton in stratified oligotrophic open-ocean waters, they usually do not bloom (i.e. increase markedly in cell concentrations) in response to episodic nutrient supplies (Mann and Chisholm, 2000). This behaviour has been explained by concomitant increases in grazing rates, implying that these grazers are able to respond very quickly to shifts in prey growth and quality. Our identification of mixotrophic predators may shed further light on this dynamic: eukaryotic mixotrophs directly exploit the same episodic supplies of dissolved nutrients as their picocyanobacterial prey, and thus could grow faster, through stimulated autotrophy, as nutrients become more abundant. As their populations grow and consume the available nutrients, they may shift towards 
phagotrophy, increasing the mortality rate of cyanobacteria, preventing bloom formation even in the face of rapid growth rates. This hypothesis is directly testable using the approach we have described.

As evidence increasingly points towards the mixotrophic capabilities of both nominally photo- and heterotrophic organisms it is becoming clear that a sharp distinction between photosynthetic and predatory lifestyles is a false dichotomy. It is likely that marine protists utilize a spectrum of trophic strategies, ranging between obligate photoautotrophic and strictly phagotrophic end members and occupying nearly all gradations in between (Sanchez-Puerta et al., 2007). Further investigations regarding other ocean sites and different depths are needed to confirm the potential importance of mixotrophy as a common metabolic strategy for grazes feeding on picocyanobacteria.

\section{Experimental procedures}

\section{Sampling and incubation conditions}

Prochlorococcus MED4 and Synechococcus WH8102 were grown for 4 days at $19^{\circ} \mathrm{C}$ under continuous cool white light

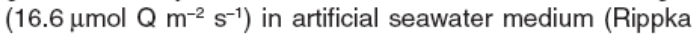
et al., 2000) amended with $6 \mathrm{mM}{ }^{13} \mathrm{C}$-sodium bicarbonate and $800 \mu \mathrm{M}{ }^{15} \mathrm{~N}$-ammonium chloride. Cells were harvested by centrifugation at $8000 \mathrm{~g}$ for $15 \mathrm{~min}$ and washed twice in unlabelled artificial seawater medium and re-suspended in the same medium. Cells were counted by flow cytometry to have an estimate of the volume of inoculum to be used in the experiment, in order to have a final concentration of picocyanobacteria similar to the concentration found in natural samples (approximately $10^{5}$ cells $\mathrm{ml}^{-1}$ ). Final isotopic enrichment of the cultures was measured by mass spectrometry at UC Davis Stable Isotope Facility using on-line combustion (Europa Integra): atom $\%{ }^{13} \mathrm{C}$ for Prochlorococcus MED4 was $98.86 \%$ and for Synechococcus WH8102 $84.20 \%$ and atom $\%{ }^{15} \mathrm{~N}$ for Prochlorococcus MED4 was $61.13 \%$ and for Synechococcus WH8102 39.83\%. These cultures were then transported overnight in the dark to the field site for use in the grazing experiments.

Samples of ocean surface water (3-5 m depth) were collected in $500 \mathrm{ml}$ acid cleaned bottles during the month of March 2006 as a part of HOT cruise 179, and inoculated with either labelled Prochlorococcus MED4 or Synechococcus WH8102 at a final concentration of $10^{5}$ cells $\mathrm{ml}^{-1}$. All shipboard incubations were performed in duplicate and analysed independently. The incubations were set in an on-deck incubator, which was constantly re-circulated with surface seawater to maintain temperature. Two samples of $200 \mathrm{ml}$ were collected at the beginning of the experiment as a control to identify the initial eukaryotic community. Samples of $250 \mathrm{ml}$ were collected from the bottles with added labelled Prochlorococcus and Synechococcus after $24 \mathrm{~h}$ of incubation. The $24 \mathrm{~h}$ period allowed enough time for the labelled isotopes to be incorporated into the nucleic acids of the grazers yet prevented both significant changes in the eukaryotic community, and potential indirect incorporation of labelled isotopes that could occur during an extended incubation. All water samples were filtered through $0.2-\mu \mathrm{m}$-pore-size membranes and preserved in RNAlater at $-80^{\circ} \mathrm{C}$ until analysis.

\section{DNA and RNA extraction, gradient fractionation and CDNA synthesis}

RNAlater was removed by washing the filters with cold $70 \%$ ethanol. DNA was extracted following Coffroth and colleagues (1992) protocol. Filters were placed in $0.5 \mathrm{ml}$ of CTAB (hexadecyltrimethyl ammonium bromide) buffer (1.4 M $\mathrm{NaCl}, 20 \mathrm{mM}$ EDTA, $100 \mathrm{mM}$ Tris-HCl pH 8.0, 0.2\% CTAB and $0.2 \%$ 2-mercapthoethanol) and the tubes were placed in a mini-bead beater (BioSpec Products, Bartlesville, OK, USA) and vortexed for $2 \mathrm{~min}$ at the maximum speed (4800 r.p.m.) to re-suspend the cells. Proteinase $\mathrm{K}$ was added to a final concentration of $0.1 \mathrm{mg} \mathrm{ml}^{-1}$ and samples were incubated at $65^{\circ} \mathrm{C}$ for $1 \mathrm{~h}$. An equal volume of chloroform was added, mixed and spun at $14000 \mathrm{~g}$ for $10 \mathrm{~min}$. The aqueous layer was transferred to a new tube and DNA was extracted with an equal volume of phenol:chloroform: isoamyl alcohol (25:24:1). Finally, DNA was precipitated by addition of 2 vols of cold $95 \%$ ethanol without addition of additional salt. Pellet was washed twice with $70 \%$ cold ethanol dried and re-suspended in water.

For RNA extraction filters were placed in $100 \mu$ l of $10 \mathrm{mM}$ Tris- $\mathrm{HCl} \mathrm{pH} \mathrm{8.0,} 4 \mu \mathrm{l}$ of RNase inhibitor (Ambion, Austin, TX, USA) and $2 \mu \mathrm{l}$ lysozyme $\left(50 \mathrm{mg} \mathrm{ml}^{-1}\right)$. Samples were incubated for $30 \mathrm{~min}$ at $37^{\circ} \mathrm{C}$. An additional $2 \mu \mathrm{l}$ of the $50 \mathrm{mg} \mathrm{ml}^{-1}$ lysozyme solution was added and the samples were incubated again for $30 \mathrm{~min}$ at $37^{\circ} \mathrm{C}$. Total RNA was immediately extracted by a mirVana RNA isolation kit (Ambion, Austin, TX, USA).

Labelled and unlabelled RNA were separated by density gradient centrifugation, performed according to the protocol of Lueders and colleagues (2004). Centrifugation media were prepared by mixing $4.5 \mathrm{ml}$ of a $2 \mathrm{~g} \mathrm{ml}^{-1}$ CsTFA stock solution (Amersham Pharmacia Biotech), up to $1 \mathrm{ml}$ of gradient buffer (GB; $0.1 \mathrm{M}$ Tris-HCl pH 8; $0.1 \mathrm{M} \mathrm{KCl} ; 1 \mathrm{mM}$ EDTA) and RNA extracts (up to $500 \mathrm{ng}$ ). Additionally, $175 \mu \mathrm{l}$ of formamide was added to centrifugation media to guarantee that RNA was denatured. The average density of all prepared gradients was checked with an AR200 digital refractometer (Leica Microsystems), and adjusted by adding small volumes of Cs salt solution or gradient buffer, if necessary. 18S rRNA was resolved in CsTFA gradients with an average density of $1.8316 \mathrm{~g} \mathrm{ml}^{-1}$ at $20^{\circ} \mathrm{C}$. Quick-Seal Polyallomer tubes, $3.9 \mathrm{ml}$ (Beckmann Instruments), were filled up with centrifugation media plus sample, and centrifuged in an Optima TLX ultracentrifuge using a TLN100 vertical rotor (Beckmann Instruments). Centrifugation conditions were $>60 \mathrm{~h}$ at 61000 r.p.m. (131 000 g).

Centrifuged gradients were fractionated from bottom to top into 12 equal fractions $(-400 \mu \mathrm{l})$. A precisely controlled flow rate was achieved by displacing the gradient medium with water at the top of the tube using a syringe pump (Harvard Apparatus). The density of $15 \mu \mathrm{l}$ from each collected fraction was determined using an AR200 digital refractometer (Leica Microsystems). Total RNA was precipitated with 1 vol. of isopropanol. Precipitates from gradient fractions were washed once with $70 \%$ ethanol and re-suspended in $25 \mu \mathrm{l}$ of EB for 
subsequent determination of total RNA using RiboGreen (Molecular Probes, Invitrogen, Carlsbad, CA, USA) assays.

Primers for $18 \mathrm{~S}$ rRNA eukaryotic genes were designed using the Design Probes tool from the ARB software (Ludwig et al., 2004): EukF (5'-GGGTTCGATTCCGGAGAG-3') EukR (5'-CCGTGTTGAGTCAAATT-3') (Integrated DNA Technologies Coralville, IA, USA). The database used contained 27887 complete sequences, all of eukaryotic origin. EukF primer matched 19378 sequences with 0 mismatches and 23459 sequences with one mismatch. EukR primer matched 25739 sequences with 0 mismatches and 27447 sequences with one mismatch. They were tested in two cultures of Cafeteria, two cultures of Paraphysomonas and one culture of Dullaniella, given in all cases the expected-size PCR product of approximately $830 \mathrm{bp}$.

Total RNA (0.5-5 ng) from fractions containing highly labelled and unlabelled RNA was reverse transcribed with the specific primers using the ThermoScript RT-PCR system (Invitrogen, Carlsbad, CA, USA). Reverse transcription was performed for $2 \mathrm{~h}$ at $50^{\circ} \mathrm{C}$.

PCR reactions were performed using Taq DNA polymerase from NEB and primers at $2 \mu \mathrm{M}$ concentration. After $5 \mathrm{~min}$ at $95^{\circ} \mathrm{C}, 35$ cycles of denaturation $\left(95^{\circ} \mathrm{C}, 45 \mathrm{~s}\right)$, annealing $\left(52^{\circ} \mathrm{C}, 1 \mathrm{~min}\right)$, elongation $\left(72^{\circ} \mathrm{C}, 1 \mathrm{~min}\right)$ and a final elongation step $\left(72^{\circ} \mathrm{C}, 10 \mathrm{~min}\right)$ were run in a MJ Research PTC 100 Thermal Cycler. PCR products were cleaned up using a QIAquick PCR purification kit (Qiagen, Valencia, CA, USA) and cloned into either TOPO TA cloning vector (Invitrogen, Carlsbad, CA, USA) or pGEM-T cloning vector (Promega, Madison, WI, USA). Inserts were sequenced either at Genaissance Pharmaceuticals (New Haven, CT; now Cogenics, MA, USA) using primers for the T7 promoter region or in house using the same primer and the BigDye sequencing kit (Applied Biosystems, Foster City, CA, USA) at 1 min denaturation and 25 cycles of $95^{\circ} \mathrm{C}-30 \mathrm{~s}, 50^{\circ} \mathrm{C}-20 \mathrm{~s}, 60^{\circ} \mathrm{C}-4 \mathrm{~min}$, and finally held at $4^{\circ} \mathrm{C}$. The reactions were then purified by ethanol precipitation and run on an ABI PRISM 3730 (Applied Biosystems) capillary DNA sequencer.

16S rRNA genes from bacteria present in the heavy fractions were cloned and sequenced using universal primers $9 \mathrm{~F}$ (5'-GAGTTTGATYMTGGCTC) and 1509R (5'-GYTACCTT GTTACGACTT) (Integrated DNA Technologies Coralville, IA, USA). PCR and cloning were performed as described above but elongation at $72^{\circ} \mathrm{C}$ was extended to $2 \mathrm{~min}$. Fragments were sequenced using the ABIPRISM BigDye Terminator v3.1 Cycle Sequencing Kit (Applied Biosystems, Warrington, UK) and primers for the T7 promoter region.

\section{Taxonomic affiliation and phylogenetic analysis}

Vector contamination was assessed using VecScreen (http:// www.ncbi.nlm.nih.gov/NecScreen/VecScreen.html). On the basis of the evaluation by the CHECK_CHIMERA program of the Ribosomal Database Project (Maidak et al., 2001) only sequences that showed no evidence for potential chimeric gene artefacts were analysed.

Preliminary taxonomic affiliation of the sequences was determined using BLASTN against the GenBank nr database (March 2005). Phylogenetic analysis was based on partial sequences trimmed to the shortest common denominator. A first analysis to confirm the taxonomic affiliation of the sequences, and have a raw picture of the overall phylogenetic tree, was performed using ARB software. Sequences were aligned against the eukaryotic database (SSRef release 90 12.05.2007, SILVA database project http://www.arbsilva.de/ with 27887 pre-aligned sequences) (Pruesse et al., 2007) in the ARB software version 07.02.20 (Ludwig et al., 2004) and performed using the Fast Alignment tool. Alignments were edited manually and sequences were added to the backbone tree using ARB's 'Parsimony insertion' feature.

For maximum likelihood (ML), neighbour joining (NJ)distance and maximum parsimony (MP) analyses, alignments were generated using MAFFT (Katoh et al., 2002; 2005) and edited manually using Sequence Alignment Editor v2.0 (http://tree.bio.ed.ac.uk/software/seal/). Maximum parsimony analysis was performed using the 'fast' stepwiseaddition algorithm in PAUP 4.0b10 (Altivec) with 1000 bootstraps replicates. For each alignment the best DNA substitution model was evaluated using MrModeltest 2.2 (Nylander, 2004), which ranked General Time Reversiblegamma-Proportion invariant $(\mathrm{GTR}+\mathrm{g}+\mathrm{l})$ best model in all cases. Maximum likelihood analysis was performed using the software PHYML_v2.4.4 (Guindon and Gascuel, 2003) and GTR as a substitution model with 100 bootstraps replicates. Neighbour joining-distance analysis was performed using PAUP 4.0b10 (Altivec) using the also GTR as a substitution model, with 1000 bootstraps replicates, and the values of Gamma-shape and proportion of invariable sites estimated by PHYML. Trees were visualized and plotted using NJPlot v2.1 (Perriere and Gouy, 1996).

\section{T-RFLP analysis}

Fluorescently labelled PCR products for the T-RFLP analysis were generated by the PCR protocol described above, using a FAM-labelled forward primer. PCR products were digested with the restriction endonucleases Hhal and Rsal (New England Biolabs, Ipswich, MA, USA). The resulting fluorescent terminal fragments were resolved and analysed at the Roy J. Carver Biotechnology Center (University of Illinois at UrbanaChampaign) using an ABI Prism 3730xl Analyser automated sequencer, and GeneMapper version 3.7 software.

Clustering of the different T-RFLP profiles was performed using the Self-Organizing Tree Algorithm (SOTA) from the GEPAS 4.0 (GEPAS website http://www.gepas.org).

\section{Chloroplast 165 rRNA analysis}

Labelled fractions from both Prochlorococcus and Synechococcus grazers were tested for the presence of 16S rRNA chloroplast sequences. Specific oligonucleotides against chloroplast sequences (SSRef release 90 12.05. 2007, SILVA database project http://www.arb-silva.de/) were design using the Design Probes tool from the ARB software (Ludwig et al., 2004). Although a total of 16 sets of primers were used in the experiment (Table S1), only the set of primers $15 \mathrm{~F}\left(5^{\prime}\right.$ TTAACTCAAGTG GCGGACGG) and 15R (AGTGTTAG TAATAGCCCAGTA) gave a PCR product long enough to be sequenced. PCR reactions were performed using Taq DNA polymerase from NEB and primers at $2 \mu \mathrm{M}$ concentration. After $5 \mathrm{~min}$ at $95^{\circ} \mathrm{C}, 40$ cycles of denaturation $\left(95^{\circ} \mathrm{C}, 45 \mathrm{~s}\right)$, 
annealing $\left(56^{\circ} \mathrm{C}, 1 \mathrm{~min}\right)$, elongation $\left(72^{\circ} \mathrm{C}, 1 \mathrm{~min}\right)$ and a final elongation step $\left(72^{\circ} \mathrm{C}, 10 \mathrm{~min}\right)$ were run in a MJ Research PTC 100 Thermal Cycler. PCR products were clean up using a QIAquick PCR purification kit (Qiagen, Valencia, CA, USA) and cloned into TOPO TA cloning vector (Invitrogen, Carlsbad, CA, USA) and sequenced as described above.

\section{Nucleotide sequence accession numbers}

Ribosomal RNA sequences have been deposited at GenBank/EMBL under Accession Nos EF695076-EF695247 and EU499951-EU500232.

\section{Acknowledgements}

This research was supported by grants from the National Science Foundation Biological Oceanography Program, the Gordon and Betty Moore Foundation Marine Microbiology Program and the Department of Energy GTL Program. Jacob Waldbauer was supported in part by an NSF Graduate Fellowship. We thank Marcia Osborne for helpful discussions and comments, which improved the manuscript. We also thank Edward F. DeLong for access to the ABI PRISM 3730 (Applied Biosystems) capillary DNA sequencer in his laboratory.

\section{References}

Adl, S.M., Simpson, A.G.B., Farmer, M.A., Andersen, R.A., Anderson, O.R., Barta, J.R., et al. (2005) The new higher level classification of eukaryotes with emphasis on the taxonomy of protists. J Eukaryot Microbiol 52: 399-451.

Anderson, M.R., and Rivkin, R.B. (2001) Seasonal patterns in grazing mortality of bacterioplankton in polar oceans: a bipolar comparison. Aquat Microb Ecol 25: 195-206.

An-Yi, T., Chiang, K.-P., Chan, Y.-F., Lin, Y.-C., and Chang, J. (2007) Pigmented nanoflagellates in the coastal western subtropical Pacific are important grazers on Synechococcus populations. J Plankton Res 29: 71-77.

Arenovski, A.L., Lim, E.L., and Caron, D.A. (1995) Mixotrophic nanoplankton in oligotrophic surface waters of the Sargasso Sea may employ phagotrophy to obtain major nutrients. J Plankton Res 17: 801-820.

Bertilsson, S., Berglund, O., Karl, D.M., and Chisholm, S.W. (2003) Elemental composition of marine Prochlorococcus and Synechococcus: implications for the ecological stoichiometry of the sea. Limnol Oceanogr 48: 1721-1731.

Carlson, C.A. (2002) Production and removal processes. In Biogeochemistry of Marine Dissolved Organic Matter. Hansell, D.A., and Carlson, C.A. (eds). San Diego: Academic Press, pp. 91-151.

Caron, D.A. (2000) Symbiosis and Mixotrophic among Pelagic Microorganism. New York, USA: Wiley-Liss.

Cavalier-Smith, T., and Chao, E.E.Y. (2006) Phylogeny and megasystematics of phagotrophic heterokonts (kingdom Chromista). J Mol Evol 62: 388-420.

Chisholm, S.W., Olson, R.J., Zettler, E.R., Waterbury, J.B., Goericke, R., and Welschmeyer, N. (1988) A novel freeliving prochlorophyte occurs at high cell concentrations in the oceanic euphotic zone. Nature 334: 340-343.
Cho, B.C., Na, S.C., and Choi, D.H. (2000) Active ingestion of fluorescently labeled bacteria by mesopelagic heterotrophic nanoflagellates in the East Sea, Korea. Mar Ecol Prog Ser 206: 23-32.

Christaki, U., Jacquet, S., Dolan, J.R., Vaulot, D., and Rassoulzadegan, F. (1999) Growth and grazing on Prochlorococcus and Synechococcus by two marine ciliates. Limnol Oceanogr 44: 52-61.

Christaki, U., Vazquez-Dominguez, E., Courties, C., and Lebaron, P. (2005) Grazing impact of different heterotrophic nanoflagellates on eukaryotic (Ostreococcus tauri) and prokaryotic picoautotrophs (Prochlorococcus and Synechococcus). Environ Microbiol 7: 1200-1210.

Chrzanowski, T.H., and Símek, K. (1990) Prey-size selection by freshwater fagellated protozoa. Limnol Oceanogr 35: 1424-1436.

Coffroth, M.A., Lasker, H.R., Diamond, M.E., Bruenn, J.A., and Bermingham, E. (1992) DNA fingerprints of a gorgonian coral: a method for detecting clonal structure in a vegetative species. Mar Biol 114: 317-325.

Countway, P.D., Gast, R.J., Savai, P., and Caron, D.A. (2005) Protistan diversity estimates based on 18S rDNA from seawater incubations in the western North Atlantic. $J$ Eukaryot Microbiol 52: 95-106.

Countway, P.D., Gast, R.J., Dennett, M.R., Savai, P., Rose, J.M., and Caron, D.A. (2007) Distinct protistan assemblages characerize the euphotic zone and deep sea $(2500$ $\mathrm{m}$ ) of the western North Atlantic (Sargasso Sea and Gulf Stream). Environ Microbiol 9: 1219-1232.

Dollhopf, S.L., Hashsham, S.A., and Tiedje, J.M. (2001) Interpreting 16S rDNA T-RFLP data: application of selforganizing maps and principal component analysis to describe community dynamics and convergence. Microb Ecol 42: 495-505.

Fuhrman, J.A., and Campbell, L. (1998) Marine ecology microbial microdiversity. Nature 393: 410-411.

Fuller, N.J., Campbell, C., Allen, D.J., Pitt, F.D., Zwirglmaierl, K., Le Gall, F., et al. (2006) Analysis of photosynthetic picoeukaryote diversity at open ocean sites in the Arabian Sea using a PCR biased towards marine algal plastids. Aquat Microb Ecol 43: 79-93.

del Giorgio, P.A., Gasol, J.M., Vaque, D., Mura, P., Agusti, S., and Duarte, C.M. (1996) Bacterioplankton community structure: protists control net production and the proportion of active bacteria in a coastal marine community. Limnol Oceanogr 41: 1169-1179.

Gonzalez, J.M., Sherr, E.B., and Sherr, B.F. (1990) Sizeselective grazing on bacteria by natural assemblages of estuarine flagellates and ciliates. App/ Environ Microbiol 56: 583-589.

González, J.M., Iriberri, J., Egea, L., and Barcina, I. (1990) Differential rates of digestion of bacteria by freshwater and marine phagotrophic protozoa. Appl Environ Microbiol 56: 1851-1857.

Guillou, L., Jacquet, S., Chretiennot-Dinet, M.J., and Vaulot, D. (2001) Grazing impact of two small heterotrophic flagellates on Prochlorococcus and Synechococcus. Aquat Microb Ecol 26: 201-207.

Guindon, S., and Gascuel, O. (2003) A simple, fast, and accurate algorithm to estimate large phylogenies by maximum likelihood. Syst Biol 52: 696-704. 
Hall, J.A., Barret, D.P., and James, M.R. (1993) The importance of phytoflagellates, heterotrophic flagellate and ciliate grazing on bacteria and picophytoplankton sized prey in a coastal marine environment. J Plankton Res 15: 1075-1086.

Hansen, P.J., and Hjorth, M. (2002) Growth and grazing responses of Chrysochromulina ericina (Prymnesiophyceae): the role of irradiance, prey concentration and pH. Mar Biol 141: 975-983.

Hansen, P.J., and Nielsen, T.G. (1997) Mixotrophic feeding of Fragilidium subglobosum (Dinophyceae) on three species of Ceratium: effects of prey concentration, prey species and light intensity. Mar Ecol Prog Ser 147: 187196.

Ishii, N., Takeda, H., Doi, M., Fuma, S., Miyamoto, K., Yanagisawa, K., and Kawabata, Z. (2002) A new method using enhanced green fluorescent protein (EGFP) to determine grazing rate on live bacterial cells by protists. Limnology 3 : 47-50.

Jones, R.I. (2000) Mixotrophy in planktonic protists: an overview. Freshw Biol 45: 219-226.

Jurgens, K., and Matz, C. (2002) Predation as a shaping force for the phenotypic and genotypic composition of planktonic bacteria. Antonie Van Leeuwenhoek 81: 413434.

Jürgens, K., and Güde, H. (1994) The potential importance of grazing-resistant bacteria in planktonic systems. Mar Ecol Prog Ser 112: 169-188.

Katoh, K., Misawa, K., Kuma, K., and Miyata, T. (2002) MAFFT: a novel method for rapid multiple sequence alignment based on fast Fourier transform. Nucleic Acids Res 30: 3059-3066.

Katoh, K., Kuma, K., Toh, H., and Miyata, T. (2005) MAFFT version 5: improvement in accuracy of multiple sequence alignment. Nucleic Acids Res 33: 511-518.

Koton-Czaarnecka, M., and Chrost, R.J. (2003) Protozoans prefer large and metabolically active bacteria. Polish $J$ Environ Studies 12: 325-334.

Landry, M.R., Lehner-Fournier, J.M., Sundstrom, J.A., Fagerness, V.L., and Selph, K.E. (1991) Discrimination between living and heat-killed prey by a marine zooflagellate, Paraphysomonas vestita (Stokes). J Exp Mar Biol Ecol 146: 139.

Lomas, M.W., Glibert, P.M., Clougherty, D.A., Huber, D.R., Jones, J., Alexander, J., and Haramoto, E. (2001) Elevated organic nutrient ratios associated with brown tide algal blooms of Aureococcus anophagefferens (Pelagophyceae). J Plankton Res 23: 1339-1344.

Ludwig, W., Strunk, O., Westram, R., Richter, L., Meier, H., Yadhukumar, et al. (2004) ARB: a software environment for sequence data. Nucleic Acids Res 32: 1363-1371.

Lueders, T., Manefield, M., and Friedrich, M.W. (2004) Enhanced sensitivity of DNA- and rRNA-based stable isotope probing by fractionation and quantitative analysis of isopycnic centrifugation gradients. Environ Microbiol 6: 73-78.

Lueders, T., Kindler, R., Miltner, A., Friedrich, M.W., and Kaestner, M. (2006) Identification of bacterial micropredators distinctively active in a soil microbial food web. Appl Environ Microbiol 72: 5342-5348.

Maidak, B.L., Cole, J.R., Lilburn, T.G., Parker, C.T., Jr,
Saxman, P.R., Farris, R.J., et al. (2001) The RDP-II (Ribosomal Database Project). Nucleic Acids Res 29: 173174.

Manefield, M., Whiteley, A.S., Griffiths, R.I., and Bailey, M.J. (2002) RNA stable isotope probing, a novel means of linking microbial community function to phylogeny. Appl Environ Microbiol 68: 5367-5373.

Mann, E.L., and Chisholm, S.W. (2000) Iron limits the cell division rate of Prochlorococcus in the eastern equatorial Pacific. Limnol Oceanogr 45: 1067-1076.

Massana, R., Guillou, L., Diez, B., and Pedros-Alio, C. (2002) Unveiling the organisms behind novel eukaryotic ribosomal DNA sequences from the ocean. Appl Environ Microbiol 68: 4554-4558.

Not, F., Gausling, R., Azam, F., Heidelberg, J.F., and Worden, A.Z. (2007) Vertical distribution of picoeukaryotic diversity in the Sargasso Sea. Environ Microbiol 9: 12331252.

Nylander, J. (2004) MrModeltest v2. Program distributed by the author. Evolutionary Biology Centre, Upssala University.

Nygaard, K., and Tobiesen, A. (1993) Bacterivory in algae a survival strategy during nutrient limitation. Limnol Oceanogr 38: 273-279.

Partensky, F., Hess, W.R., and Vaulot, D. (1999a) Prochlorococcus, a marine photosynthetic prokaryote of global significance. Microbiol Mol Biol Rev 63: 106-127.

Partensky, F., Blanchot, J., and Vaulot, D. (1999b) Differential distribution and ecology of Prochlorococcus and Synechococcus in oceanic waters: a review. Bull Inst Océanogr Monaco Special No. 19: 457-475.

Pernthaler, J. (2005) Predation on prokaryotes in the water column and its ecological implications. Nat Rev Microbiol 3: $537-546$.

Perriere, G., and Gouy, M. (1996) WWW-Query: an on-line retrieval system for biological sequence banks. Biochimie 78: 364-369.

Pruesse, E., Quast, C., Knittel, K., Fuchs, B.M., Ludwig, W., Peplies, J., and Glockner, F.O. (2007) SILVA: a comprehensive online resource for quality checked and aligned ribosomal RNA sequence data compatible with ARB. Nucleic Acids Res 35: 7188-7196.

Radajewski, S., Ineson, P., Parekh, N.R., and Murrell, J.C. (2000) Stable-isotope probing as a tool in microbial ecology. Nature 403: 646-649.

Raven, J.A. (1997) Phagotrophy in phototrophs. Limnol Oceanogr 42: 198-205.

Rippka, R., Coursin, T., Hess, W., Lichtle, C., Scanlan, D.J., et al. (2000) Prochlorococcus marinus Chisholm et al. 1992 subsp. pastoris subsp. nov. strain PCC 9511, the first axenic chlorophyll a2/b2-containing cyanobacterium (Oxyphotobacteria). Int J Syst Evol Microbio/ 50 (Part 5): 18331847.

Sanchez-Puerta, M.V., Lippmeier, J.C., Apt, K.E., and Delwiche, C.F. (2007) Plastid genes in a non-photosynthetic dinoflagellate. Protist 158: 105-117.

Shalchian-Tabrizi, K., Eikrem, W., Klaveness, D., Vaulot, D., Minge, M.A., Le Gall, F., et al. (2006) Telonemia, a new protist phylum with affinity to chromist lineages. Proc Biol Sci 273: 1833-1842.

Sherr, B.F., Sherr, E.B., and Fallon, R.D. (1987) Use of 
monodispersed, fluorescently labeled bacteria to estimate in situ protozoan bacterivory. Appl Environ Microbiol 53: 958-965.

Sherr, E.B., and Sherr, B.F. (2002) Significance of predation by protists in aquatic microbial food webs. Antonie Van Leeuwenhoek (Int J Gen Mol Microbiol) 81: 293-308.

Sherr, E.B., Rassouladegan, F., and Sherr, B.F. (1989) Bacterivory by pelagic choreotrichous ciliates in coastal waters of the NW Mediterranean Sea. Mar Ecol Prog Ser 55: 235-240.

Simek, K., and Chrzanowski, T.H. (1992) Direct and indirect evidence of size-selective grazing on pelagic bacteria by freshwater nanoflagellates. Appl Environ Microbiol 58: 3715-3720.

Stoeck, T., Zuendorf, A., Breiner, H.W., and Behnke, A. (2007) A molecular approach to identify active microbes in environmental eukaryote clone libraries. Microb Ecol 53: 328-339.

Unrein, F., Massana, R., Alonso-Saez, L., and Gasol, J.M. (2007) Significant year-round effect of small mixotrophic flagellates on bacterioplankton in an oligotrophic coastal system. Limnol Oceanogr 52: 456-469.

Worden, A.Z., and Binder, B.J. (2003) Application of dilution experiments for measuring growth and mortality rates among Prochlorococcus and Synechococcus populations in oligotrophic environments. Aquat Microb Ecol 30: 159174.

\section{Supporting information}

Additional Supporting Information may be found in the online version of this article:

Fig. S1. Phylogenetic analysis of the sequences derived from the 18S rDNA sequences in the indigenous microbial community. Unrooted phylogenetic tree inferred by maximum likelihood (ML) analysis. A total of 1090 positions used, including gaps (sequences ranging from a minimum of $585 \mathrm{bp}$ up to $893 \mathrm{bp}$ ), from an alignment of 203 partial sequences were used. Bootstrap values over $50 \%$ are indicated on the internal branches obtained from both $\mathrm{ML}$, neighbour joining-distance methods (NJ-Dis) and using maximum parsimony (MP) (in the order ML/NJ-Dist/MP). Bootstrap values $<50 \%$, or not supported at least in two of the analyses, have been omitted. The gamma distribution parameter $(\alpha)$ was estimated at 0.520 ; and the proportion of invariant sites $(I)$ was 0.015 . The scale bar indicates $10 \%$ divergence. The sequences from the duplicate biological samples are indicated as (dhot1) and (dhot2). Classification is based on Adl and colleagues (2005) and Not and colleagues (2007). All groups correspond to first and second rank according to Adl and colleagues (2005) except *Super-group and ${ }^{* *}$ Phylum (Shalchian-Tabrizi et al., 2006).

Fig. S2. Phylogenetic analysis of the sequences derived from the 18S rDNA sequences in the unlabelled fractions from the experiments (time 0 and blue circles in Fig. 2). Unrooted SSU rRNA-derived library phylogenetic tree of eukaryotes inferred by maximum likelihood (ML) analysis. A total of 1058 positions used, including gaps (sequences ranging from a minimum of $512 \mathrm{bp}$ up to $886 \mathrm{bp}$ ), from an alignment of 200 partial sequences were used. Bootstrap values over $50 \%$ are indicated on the internal branches obtained from both ML, neighbour joining-distance methods (NJ-Dis) and using maximum parsimony (MP) (in the order $\mathrm{ML} / \mathrm{NJ}$-Dist/MP). Bootstrap values $<50 \%$, or not supported at least in two of the analyses, have been omitted. The gamma distribution parameter $(\alpha)$ was estimated at 0.594; and the proportion of invariant sites $(I)$ was 0.000 . The scale bar indicates $10 \%$ divergence. The sequences coming from the duplicate biological samples is indicated as (A) and (B). Clones colour code: dark red: sequences from the time 0 sample, representing the metabolically active initial eukaryotic microbial community; purple: sequences from the unlabelled eukaryotic RNA obtained from the samples incubated with Prochlorococcus; orange: sequences from the unlabelled fraction from the samples incubated with Synechococcus. Classification is based on Adl and colleagues (2005) and Not and colleagues (2007). All groups correspond to first and second rank according to Adl and colleagues (2005) except when noted as follows: *Super-group and **Phylum (Shalchian-Tabrizi et al., 2006). ${ }^{* * *}$ Unidentified chloroplastida, BLAST results gave no clear match and the sequences did not cluster clearly with any of the second-rank groups used in the tree that could indicate the exact affiliation of the sequence.

Fig. S3. Phylogenetic analysis of the sequences derived from the labelled 18S rDNA sequences (red circles in Fig. 2) from the experimental bottles amended with labelled Prochlorococcus cells. Unrooted $18 \mathrm{~S}$ rRNA-derived library phylogenetic tree of eukaryotes inferred by maximum likelihood (ML) analysis. A total of 1145 positions used, including gaps (sequences ranging from a minimum of $545 \mathrm{bp}$ up to $980 \mathrm{bp}$ ), from an alignment of 192 partial sequences were used. Bootstrap values over $50 \%$ are indicated on the internal branches obtained from both $\mathrm{ML}$, neighbour joining-distance methods (NJ-Dis) and using maximum parsimony (MP) (in the order ML/NJ-Dist/MP). Bootstrap values $<50 \%$, or not supported at least in two of the analyses, have been omitted. The gamma distribution parameter $(\alpha)$ was estimated at 0.512 ; and the proportion of invariant sites $(I)$ was 0.000 . The scale bar indicates $10 \%$ divergence. The sequences coming from the duplicate biological samples is indicated as (A) and (B). Classification is based on Adl and colleagues (2005) and Not and colleagues (2007). All groups correspond to first and second rank according to Adl and colleagues (2005) except when noted as follows: *Super-group and ${ }^{* *}$ Phylum (ShalchianTabrizi et al., 2006). "** Unidentified stramenopiles, BLAST results gave no clear match and the sequences did not cluster clearly with any of the second-rank groups used in the tree that could indicate the exact affiliation of the sequence. Fig. S4. Phylogenetic analysis of the sequences derived from the labelled $18 \mathrm{~S}$ rDNA sequences (red circles in Fig. 2) from the experimental bottles amended with labelled Synechococcus cells. Unrooted $18 \mathrm{~S}$ rRNA-derived library phylogenetic tree of eukaryotes inferred by maximum likelihood (ML) analysis. A total of 1156 positions used, including gaps (sequences ranging from a minimum of $507 \mathrm{bp}$ up to $977 \mathrm{bp}$ ), from an alignment of 188 partial sequences were used. Bootstrap values over $50 \%$ are indicated on the internal branches obtained from both $\mathrm{ML}$, neighbour joining-distance methods (NJ-Dis) and using maximum parsimony (MP) (in the order ML/NJ-Dist/MP). Bootstrap values $<50 \%$, or not supported at least in two of the analyses, have been omitted. The gamma 
Identifying grazers of picocyanobacteria in ocean waters 525

distribution parameter $(\alpha)$ was estimated at 0.543 ; and the proportion of invariant sites $(I)$ was 0.036 . The scale bar indicates $10 \%$ divergence. The sequences coming from the duplicate biological samples is indicated as (A) and (B). Classification is based on Adl and colleagues (2005) and Not and colleagues (2007). All groups correspond to first and second rank according to Adl and colleagues (2005) except when noted as follows: *Super-group and **Phylum (ShalchianTabrizi et al., 2006). "***Unidentified stramenopiles, BLAST results gave no clear match and the sequences did not cluster clearly with any of the second-rank groups used in the tree that could indicate the exact affiliation of the sequence. Fig. S5. Phylogenetic tree 16S rRNA sequences from chloroplasts and bacteria inferred by maximum likelihood (ML) analysis. Blue: cyanobacterial 16S rRNA sequences. Green: sequences originating from the labelled fraction of the Prochlorococcus inoculation experiment. Orange: sequences originated from the labelled fraction from the Synechococcus inoculation experiment. (A) and (B) represent the two biological replicates in the experiments. A total of 724 positions were used, including gaps (sequences ranging from a minimum of $358 \mathrm{bp}$ up to $668 \mathrm{bp}$ ), from an alignment of 103 partial sequences. Bootstrap values over $50 \%$ are indicated on the internal branches obtained from $\mathrm{ML}$, neighbour joiningdistance methods (NJ-Dist) and using maximum parsimony (MP) (in the order ML/NJ-Dist/MP). Bootstrap values $<50 \%$, or not supported at least in two of the analyses, have been omitted. The proportion of invariant sites $(I)$ was 0.241 . The scale bar indicates $10 \%$ divergence. An archaeal sequence was used as out-group (Sulfolobus acidocaldarius).

Table S1. Oligonucleotides used for the amplification of 16S rRNA chloroplast genes from different groups defined based on the ARB tree (SSRef release 90 12.05.2007) for these group of sequences. $F$, forward primer. $R$, reverse primer.

Please note: Wiley-Blackwell are not responsible for the content or functionality of any supporting materials supplied by the authors. Any queries (other than missing material) should be directed to the corresponding author for the article.

(C) 2008 The Authors

Journal compilation @ 2008 Society for Applied Microbiology and Blackwell Publishing Ltd, Environmental Microbiology, 11, 512-525 
Table S1. Oligonucleotides used for the amplification of 16S rRNA chloroplast genes from different groups defined based on the ARB tree (SSRef release 90 12.05.2007) for these group of sequences. F, forward primer. R, reverse primer.

\begin{tabular}{|c|c|c|c|}
\hline $\begin{array}{l}\text { Primers' } \\
\text { set } \\
\text { number }\end{array}$ & Group targeted & Sequence & $\begin{array}{l}\text { Expected size } \\
\text { of amplified } \\
\text { product in bp }\end{array}$ \\
\hline \multirow[t]{2}{*}{1} & Zea and rel.-F & GAA GTG GTG TTT CCA GTG GC & 351 \\
\hline & Zea and rel.-R & AAA AGA AGT TCA CGA CCC GT & \\
\hline \multirow[t]{2}{*}{2} & Chlamydomonas and rel.-F & ACA CGT CAA CGC ACG AGC TG & 821 \\
\hline & Chlamydomonas and rel.-R & TAG CTA GTT GGT GGG GGT AA & \\
\hline \multirow[t]{2}{*}{3} & Anthocerus and rel.-F & TAA GGA GGG GCT TGC GTT TG & 218 \\
\hline & Anthocerus and rel.-R & GTC ATT GCT TCT TCT CTA AG & \\
\hline \multirow[t]{2}{*}{4} & Marchantia and rel.-F & CCT TTT CTC AGA GAA GAT GC & 813 \\
\hline & Marchantia and rel.-R & GCG AGG TCG CGA CCC TTT GT & \\
\hline \multirow[t]{2}{*}{5} & Spirogyra and rel.-F & TAG TCT CCA CCG CCT GGC CA & 485 \\
\hline & Spirogyra and rel.-R & GGC GGG GGA CCA CCA CTG GA & \\
\hline \multirow[t]{2}{*}{6} & Palmaria_1 and rel.-F & CGC CTT AGC TAC GAT ACT GC & 89 \\
\hline & Palmaria_- 1 and rel.-R & AGA CGA CAG CTA GGG GAG CA & \\
\hline \multirow[t]{2}{*}{7} & Ochromonas and rel.-F & CCA CCT GTG TAA GAG GCC GT & 581 \\
\hline & Ochromonas and rel.-R & GGA AGA TCT GAC GTT ACT TG & \\
\hline \multirow[t]{2}{*}{8} & Palmaria_2 and rel.-F & CTA CGA TAC TGC ACG GAT CG & 136 \\
\hline & Palmaria_2 and rel.-R & TGG GAA GAA CAC CAG AAG CG & \\
\hline \multirow[t]{2}{*}{9} & Chara and rel.-F & GCA CTG AAC GGA TCA AAT CG & 712 \\
\hline & Chara and rel.-R & AAG GAC TTG CCC TTG GGT GG & \\
\hline \multirow[t]{2}{*}{10} & Bryophyta and rel.-F & GGA GCG AAA GGA GGA ATC CA & 31 \\
\hline & Bryophyta and rel.-R & ACG CAA GCC CCT CCT TGG GT & \\
\hline \multirow[t]{2}{*}{11} & Chlorella and rel.-F & CCC CAG GCG GGA TAC TTC ACG & 160 \\
\hline & Chlorella and rel.-R & GGG AGG AAC ACC AAA GGC GA & \\
\hline \multirow[t]{2}{*}{12} & Chlorarachnion and rel.-F & AGC GAG GGG AGA GAA TGG GA & 436 \\
\hline & Chlorarachnion and rel.-R & GCC CAG AAC TTA AGG GGC AT & \\
\hline \multirow[t]{2}{*}{13} & Osmunda and rel.-F & AGC AAA AGG GAG GGA TCC GC & 398 \\
\hline & Osmunda and rel.-R & TTG ACA GCG GAC TTA AGG AG & \\
\hline \multirow[t]{2}{*}{14} & Chaetosphaeridium and rel.-F & CTT GCG TCT GAT TAT GCT AG & 175 \\
\hline & Chaetosphaeridium and rel.-R & ACC CGT AAG CTT TCT TCC T & \\
\hline \multirow[t]{2}{*}{15} & Skeletonema and rel.-F & TTA ACT CAA GTG GCG GAC GG & 650 \\
\hline & Skeletonema and rel.-R & AGT GTT AGT AAT AGC CCA GTA & \\
\hline \multirow[t]{2}{*}{16} & Euglenales and rel.-F & GGG GAG TAC GCT TGC AAA AG & 153 \\
\hline & Euglenales and rel.-R & CAT GCA CCA CCT GTG TCT AG & \\
\hline
\end{tabular}




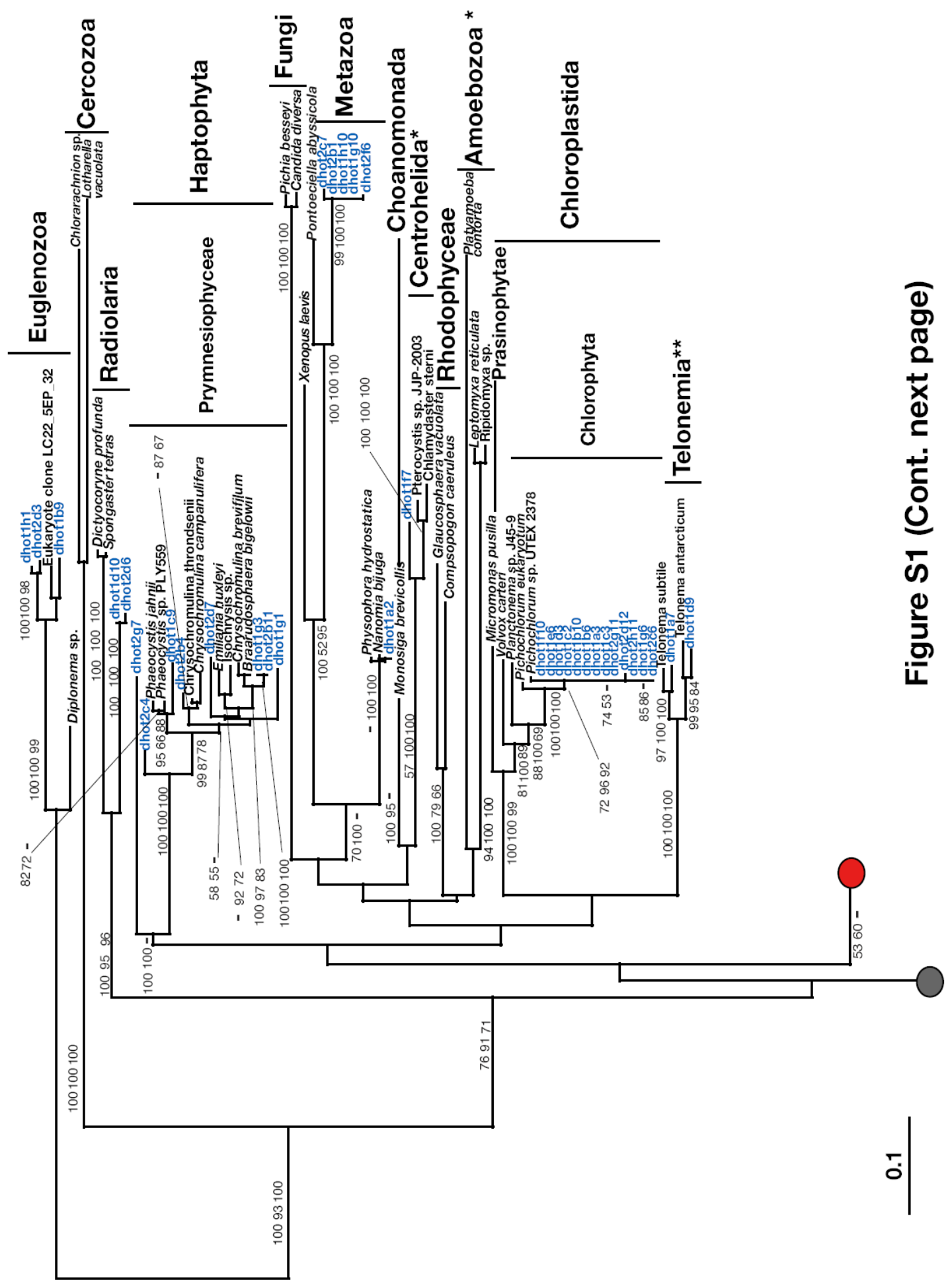




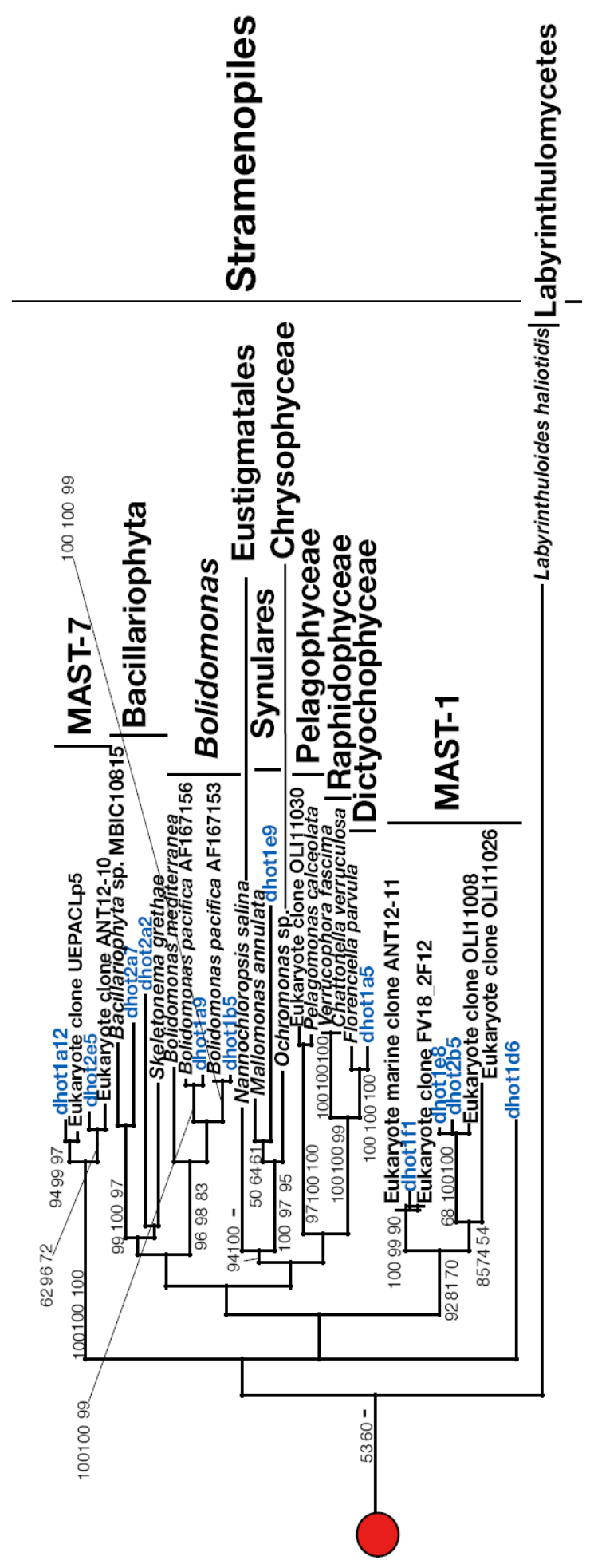

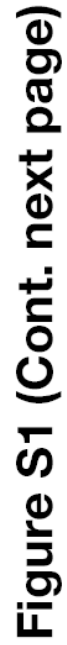

5 


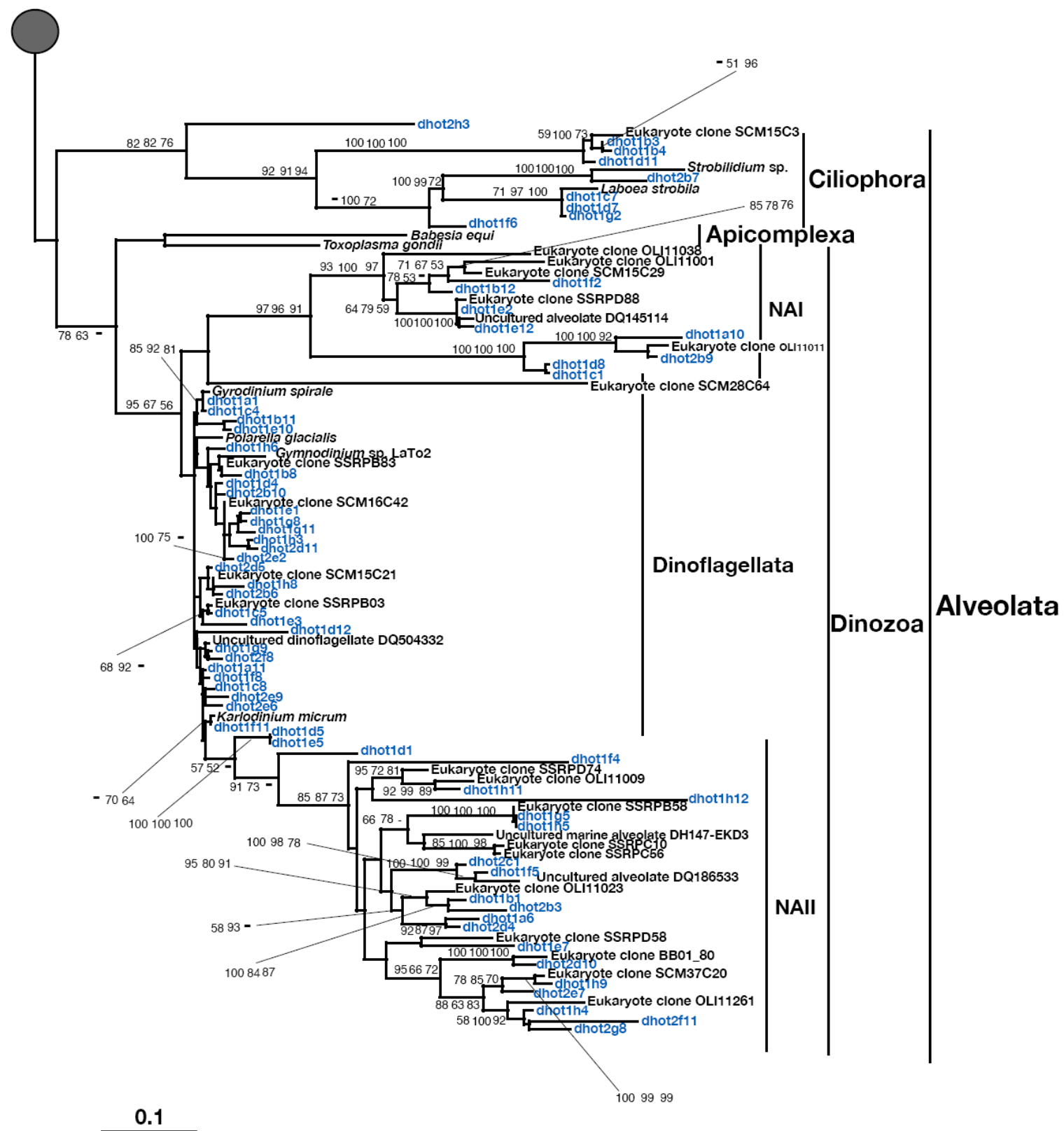

Figure S1 


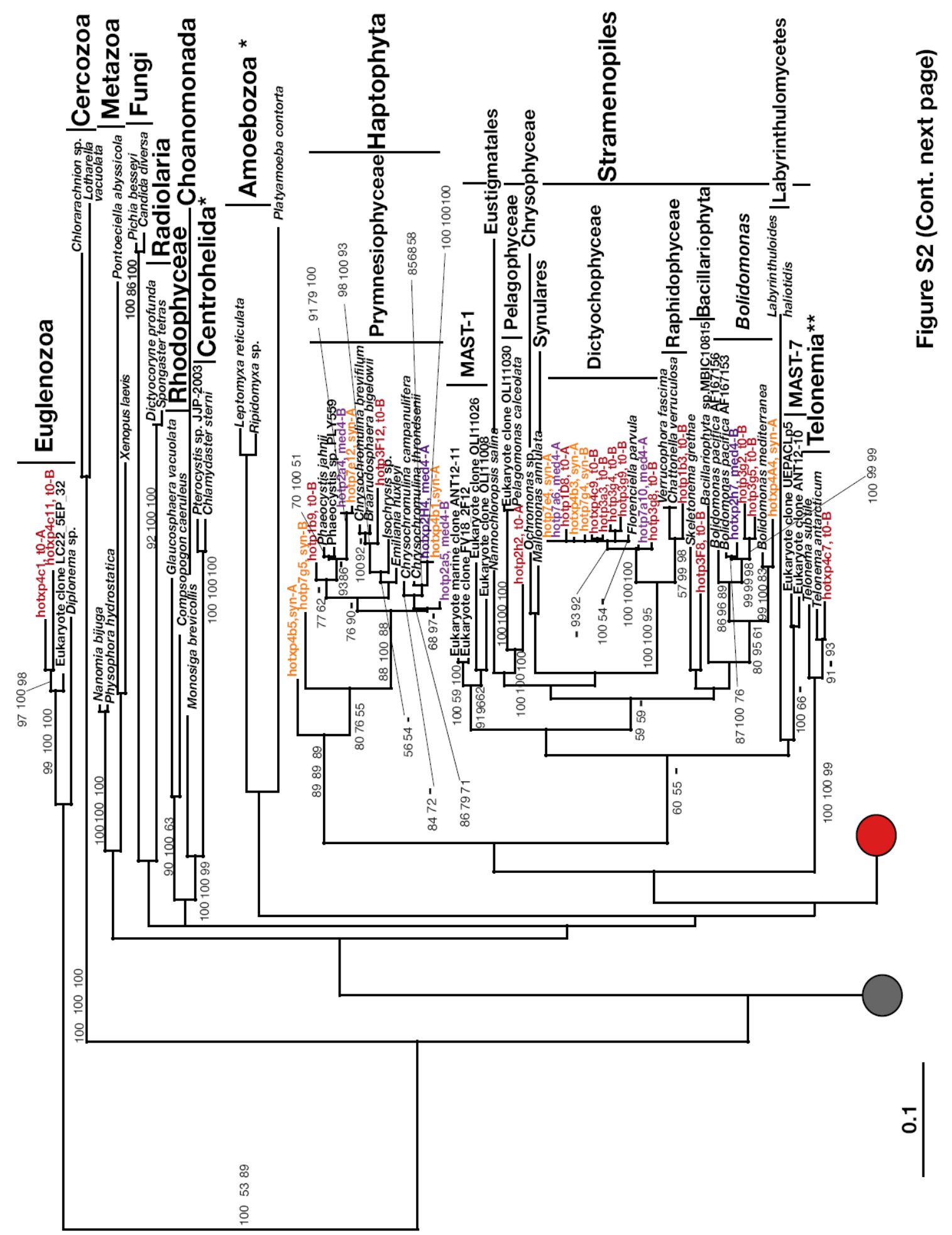




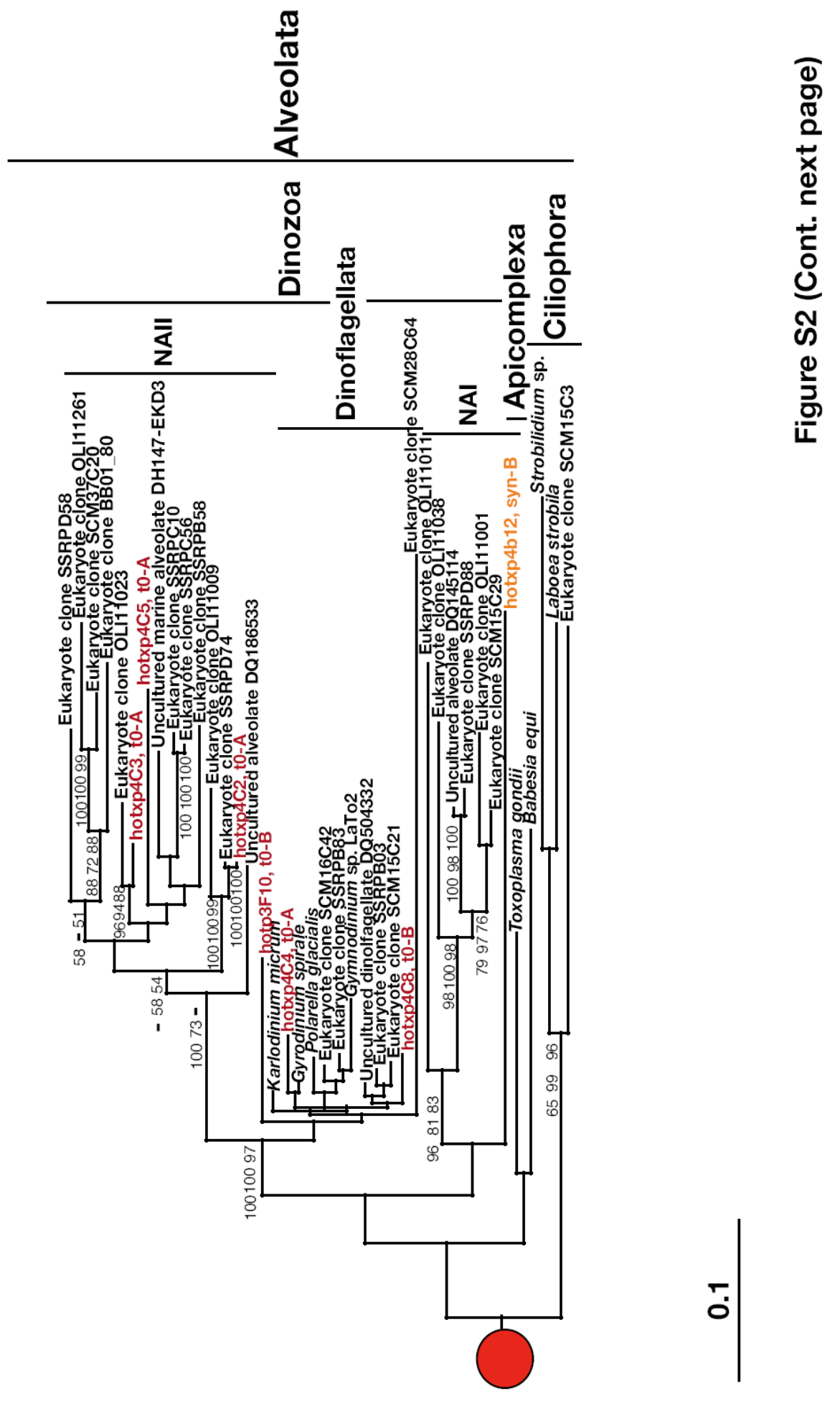




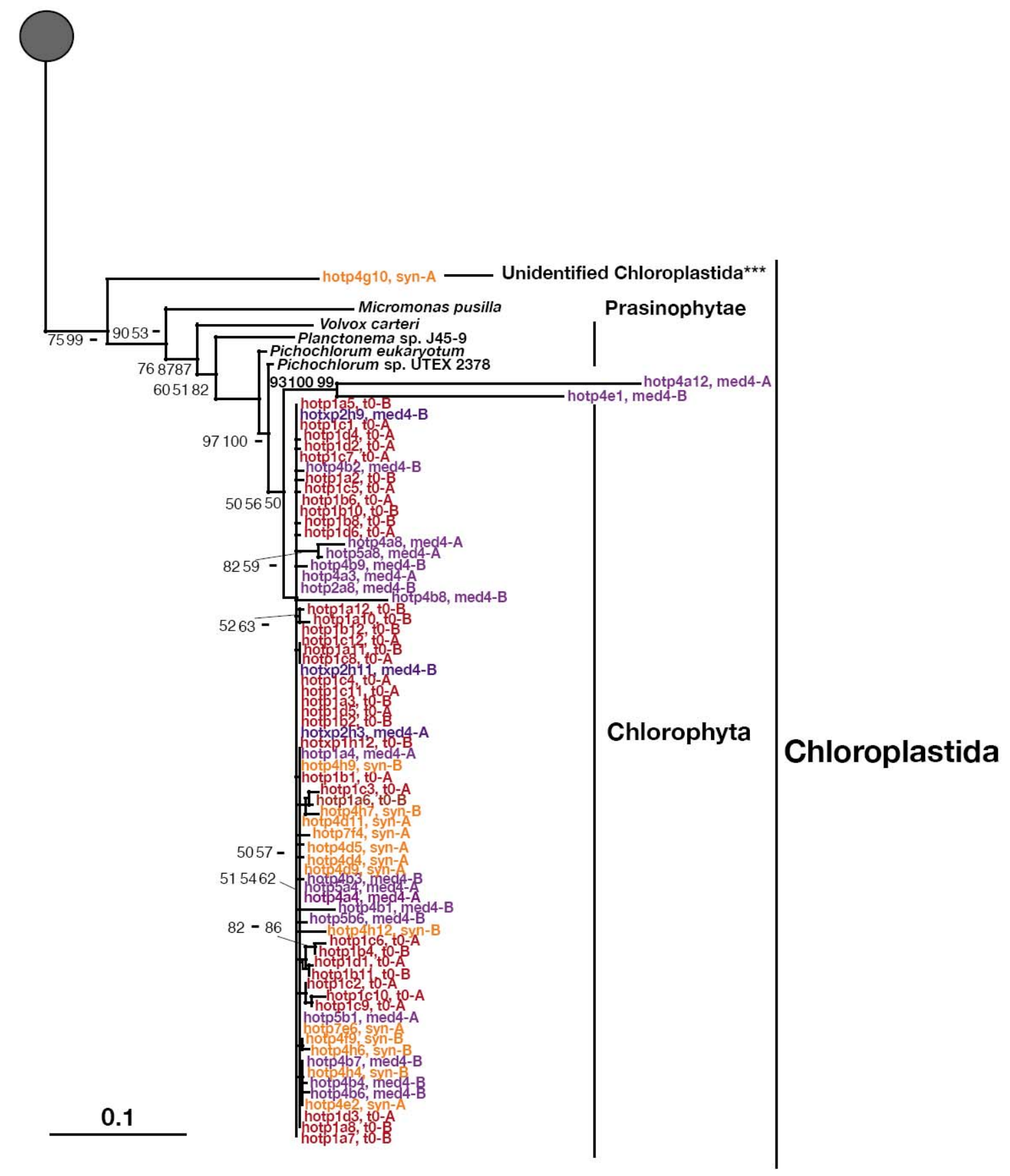

Figure S2 


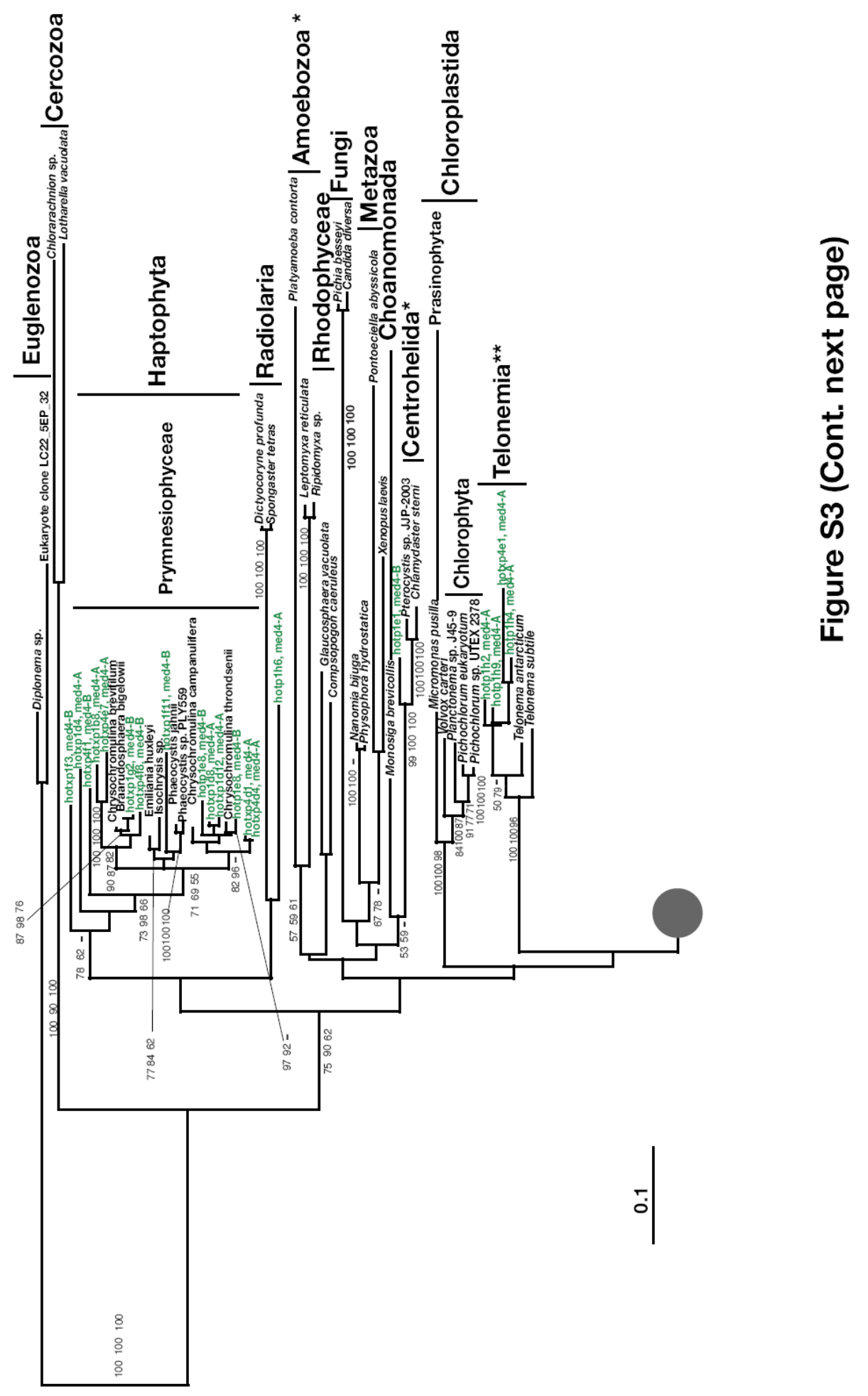




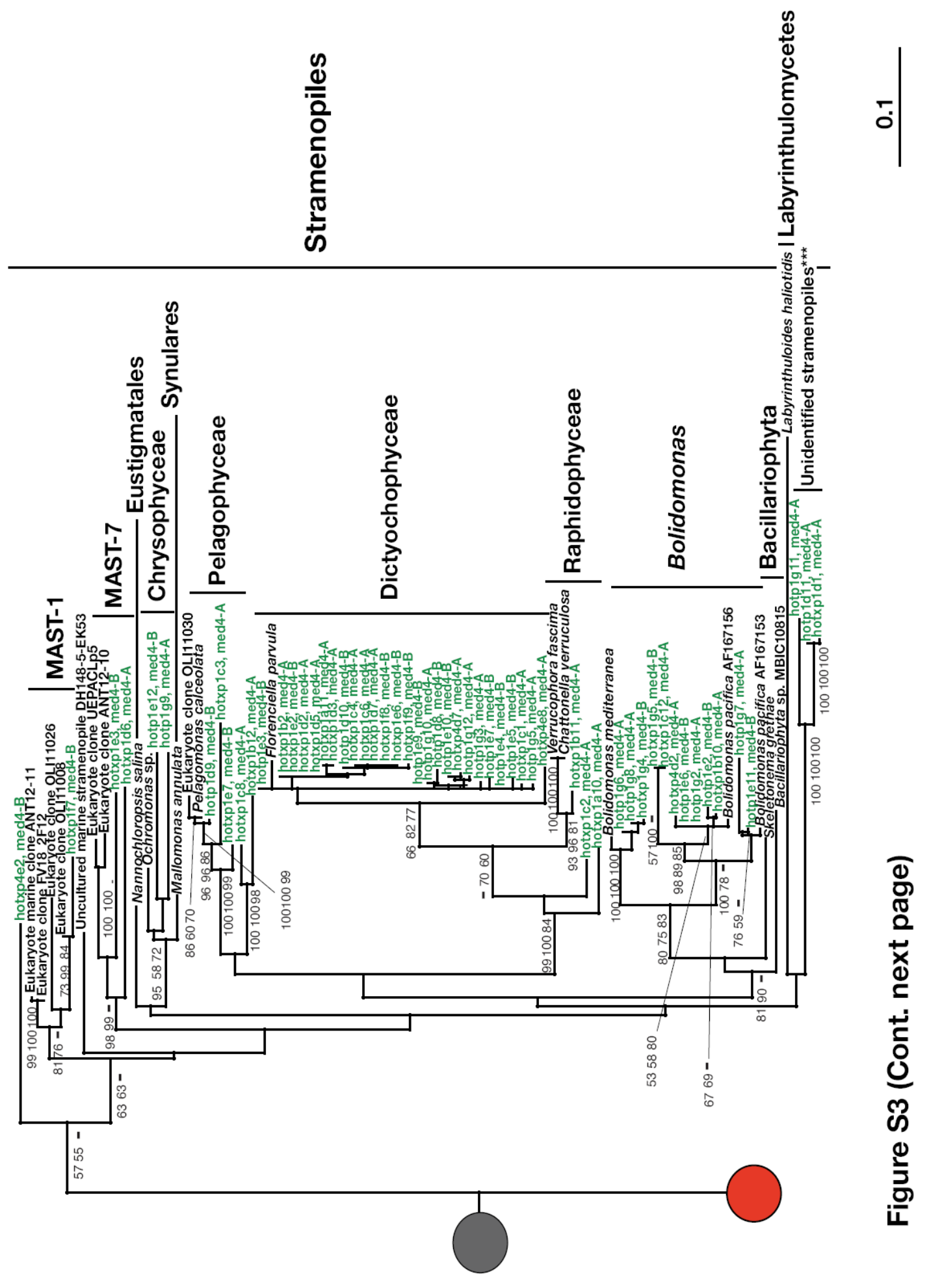




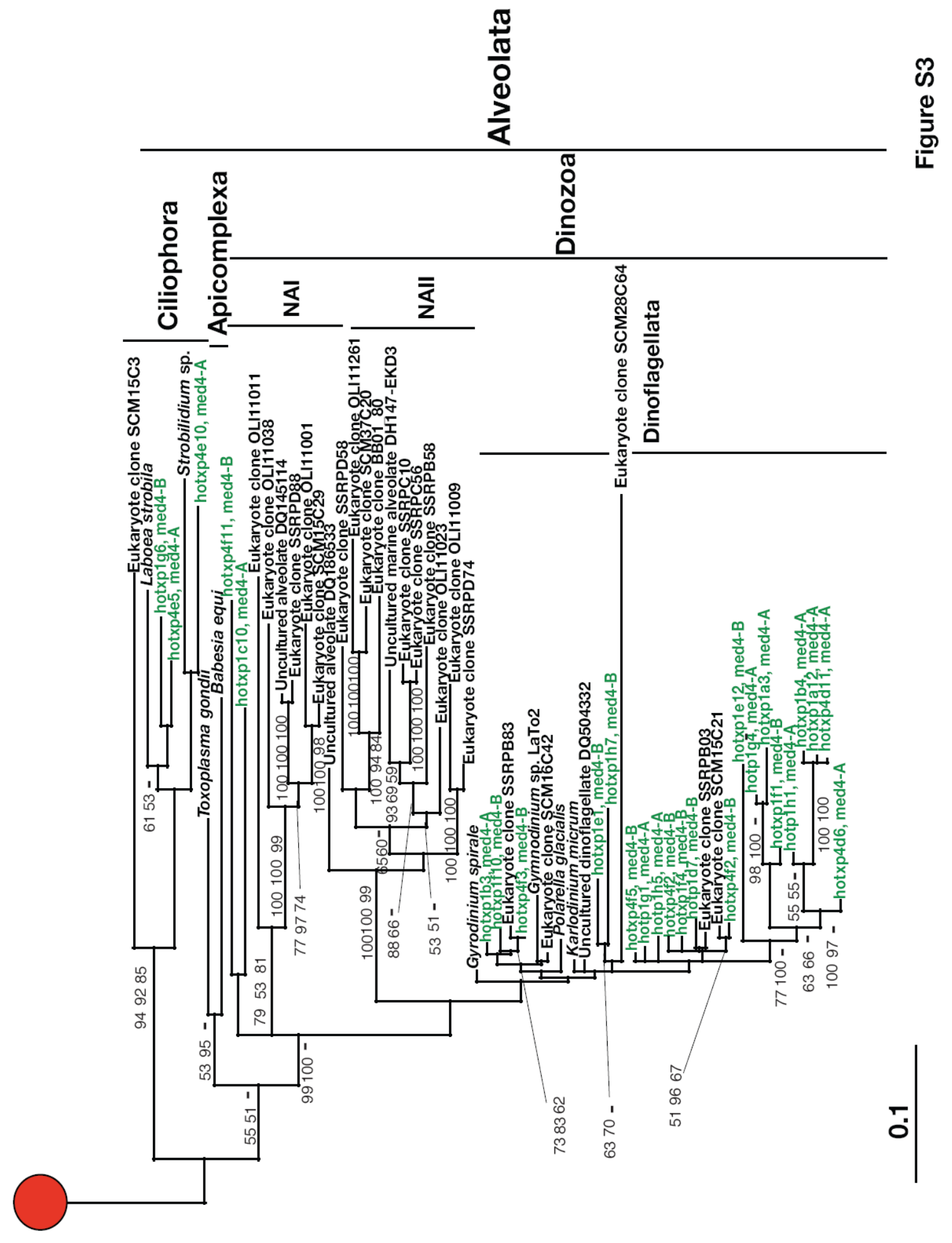




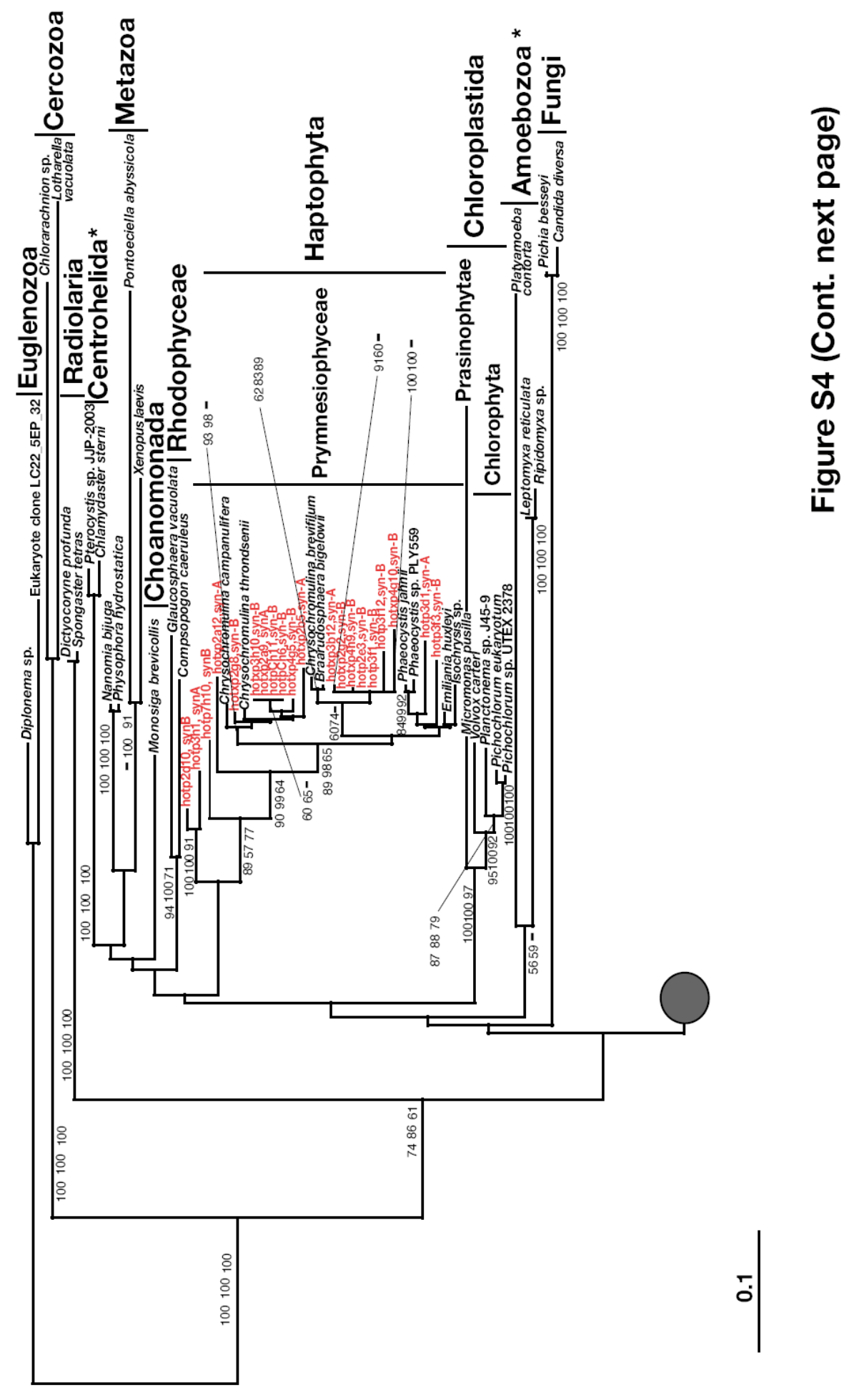




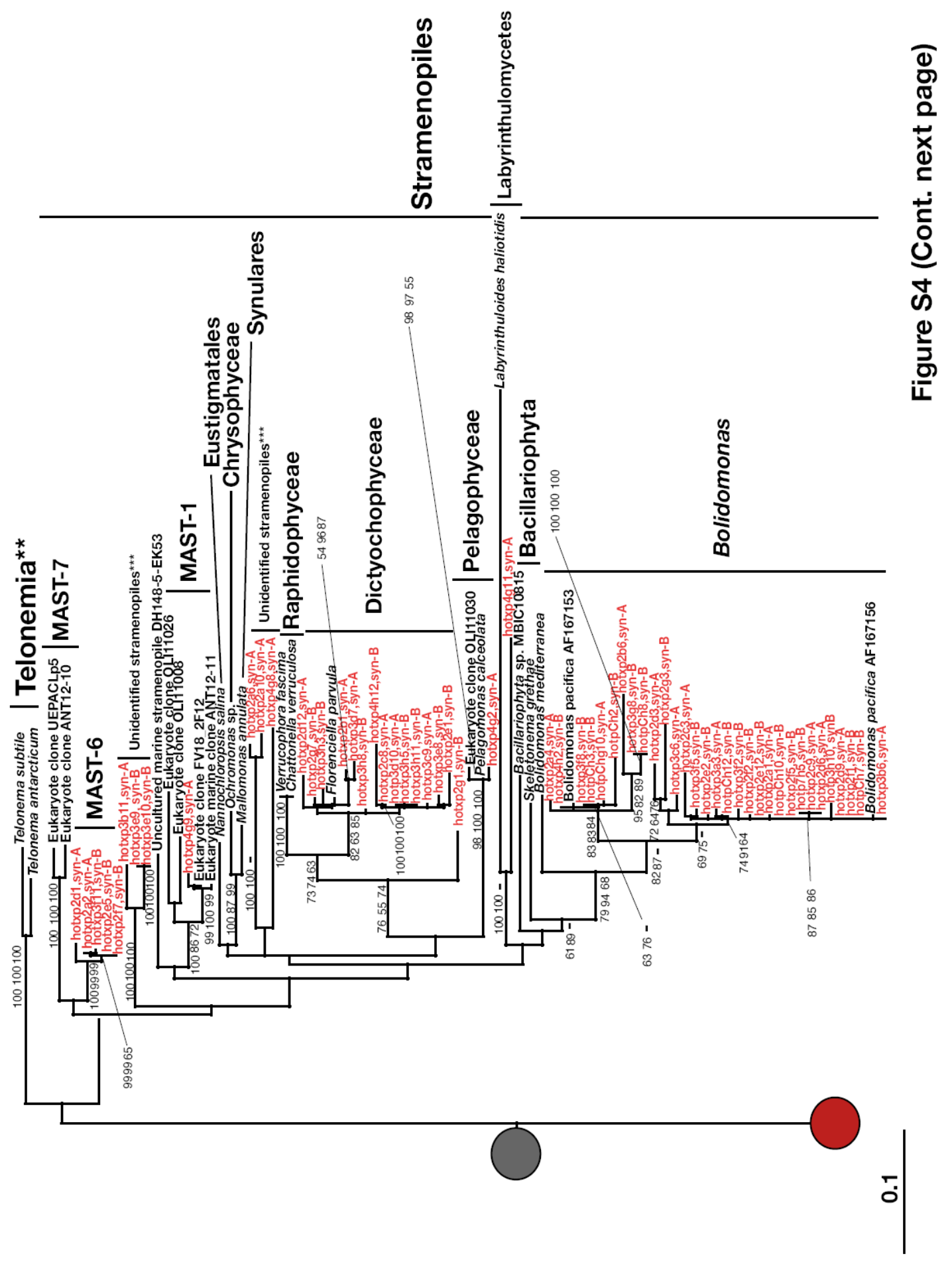




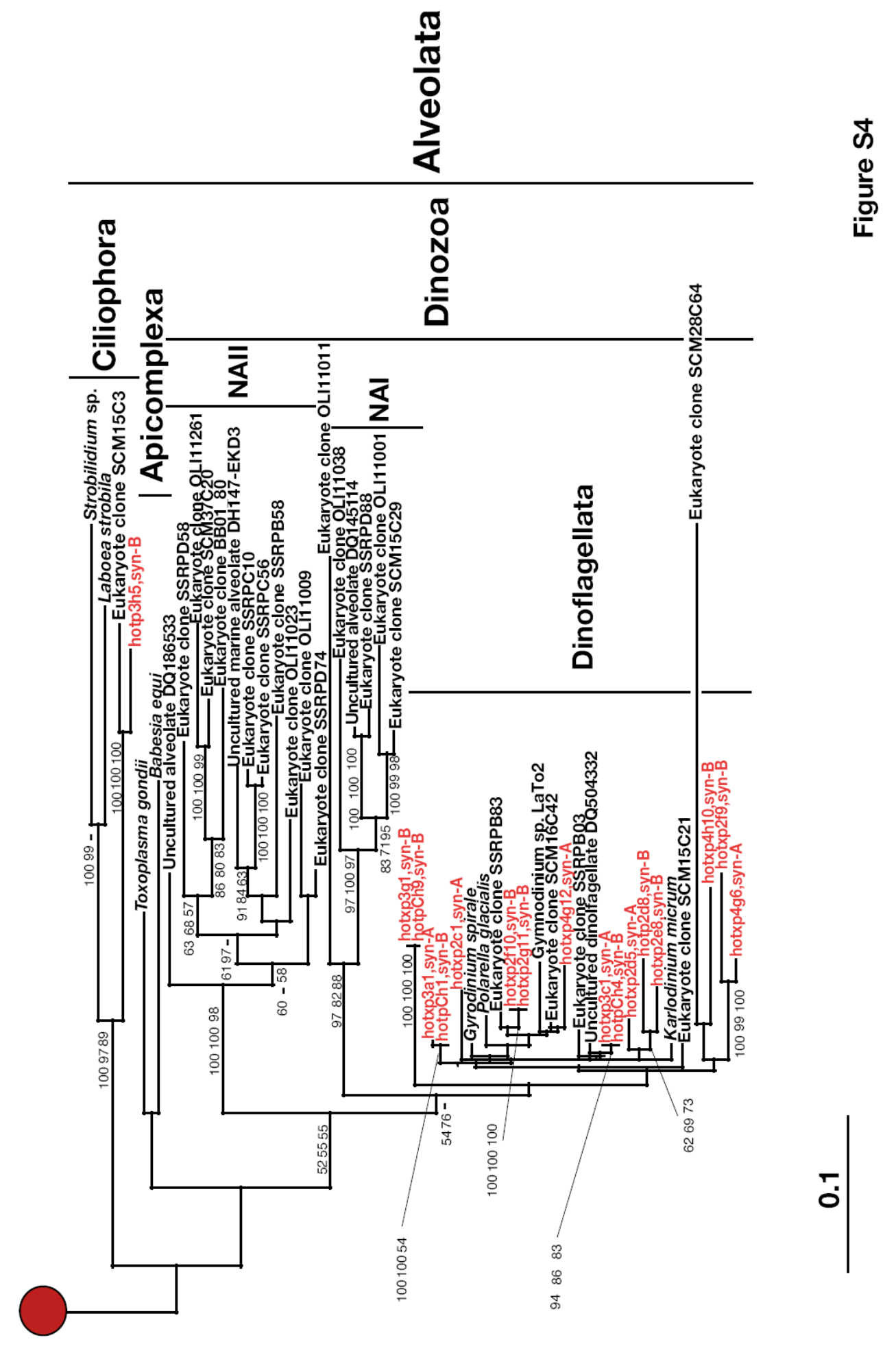




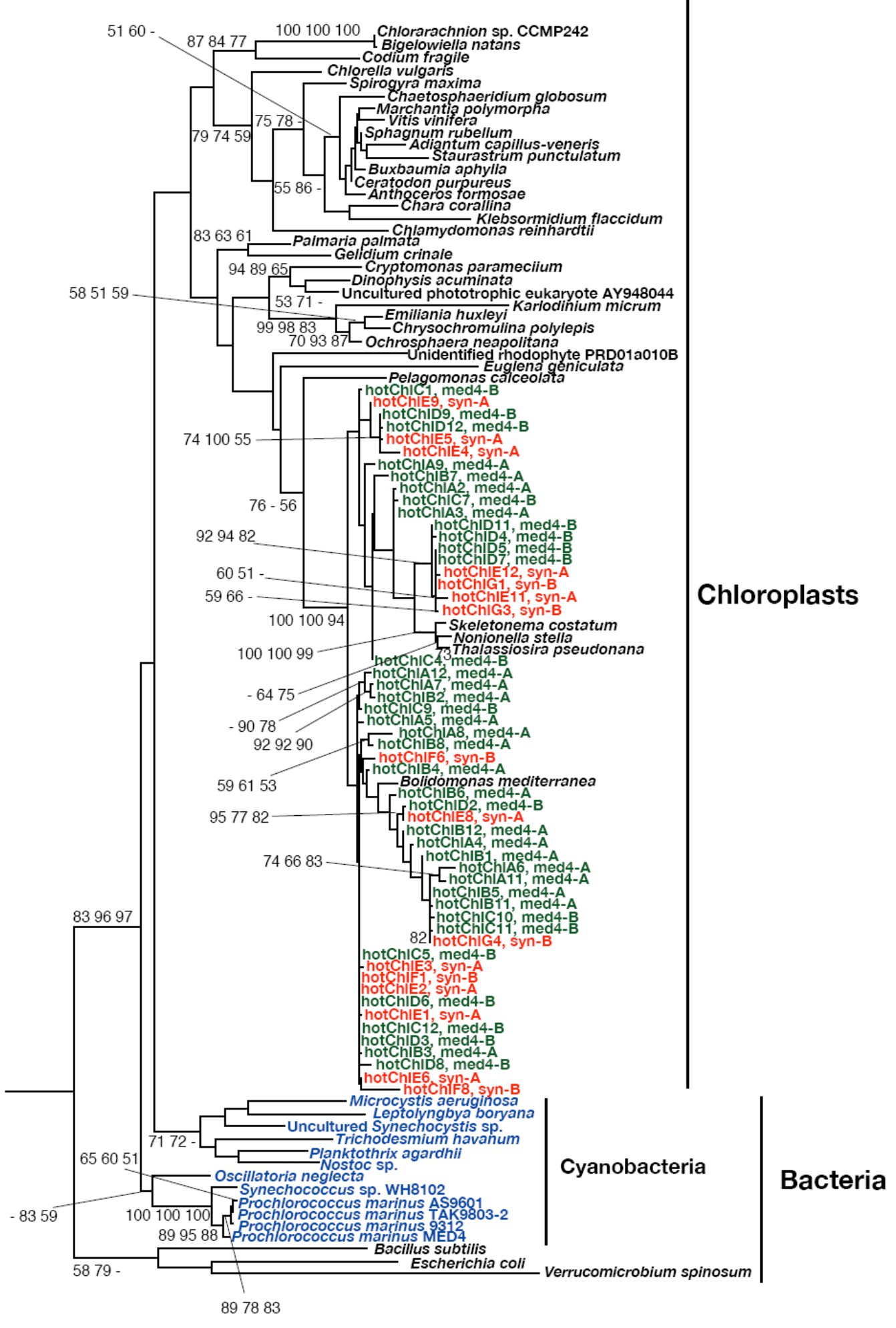

Figure S5 



\begin{abstract}
APPENDIX B
Updated analysis and comparisons of Prochlorococcus whole-genome expression in response to iron starvation, nitrogen starvation, phosphorus starvation, light stress, and phage infection
\end{abstract}

Anne Thompson ${ }^{1}$

Katherine Huang ${ }^{2}$

Sallie W. Chisholm ${ }^{2,3}$

${ }^{1}$ MIT-WHOI Joint Program in Biological Oceanography

${ }^{2}$ MIT Department of Civil and Environmental Engineering

${ }^{3}$ MIT Department of Biology 


\begin{tabular}{|c|c|c|c|c|}
\hline Experiment & Strain & Normalizations & q-value* & $\log _{2}(E / C)^{*}$ \\
\hline \multirow[t]{2}{*}{ Iron $^{1}$} & MED4 & RMA, loess & 0.01 & 1 \\
\hline & MIT9313 & RMA, loess & 0.01 & 1 \\
\hline \multirow[t]{2}{*}{ Nitrogen $^{2}$} & MED4 & RMA, loess & 0.01 & 1.5 \\
\hline & MIT9313 & RMA, loess & 0.01 & 2 \\
\hline \multirow[t]{2}{*}{ Phosphorus $^{3}$} & MED4 & RMA, loess & 0.05 & 0 \\
\hline & MIT9313 & RMA, loess & 0.05 & 0 \\
\hline Light $^{4}$ & MED4 & RMA, loess & 0.01 & 1 \\
\hline Phage $^{5}$ & MED4 & RMA & 0.01 & 1 \\
\hline \multicolumn{5}{|c|}{$\begin{array}{l}{ }^{1} \text { This study. } \\
{ }^{2} \text { Tolonen et al. } 2006 . \\
{ }^{3} \text { Martiny and Coleman et al. } 2006 . \\
{ }^{4} \text { Steglich et al. } 2006 . \\
{ }^{5} \text { Lindell et al. } 2007 \text {. } \\
\text { * Thresholds used to identify differe }\end{array}$} \\
\hline
\end{tabular}




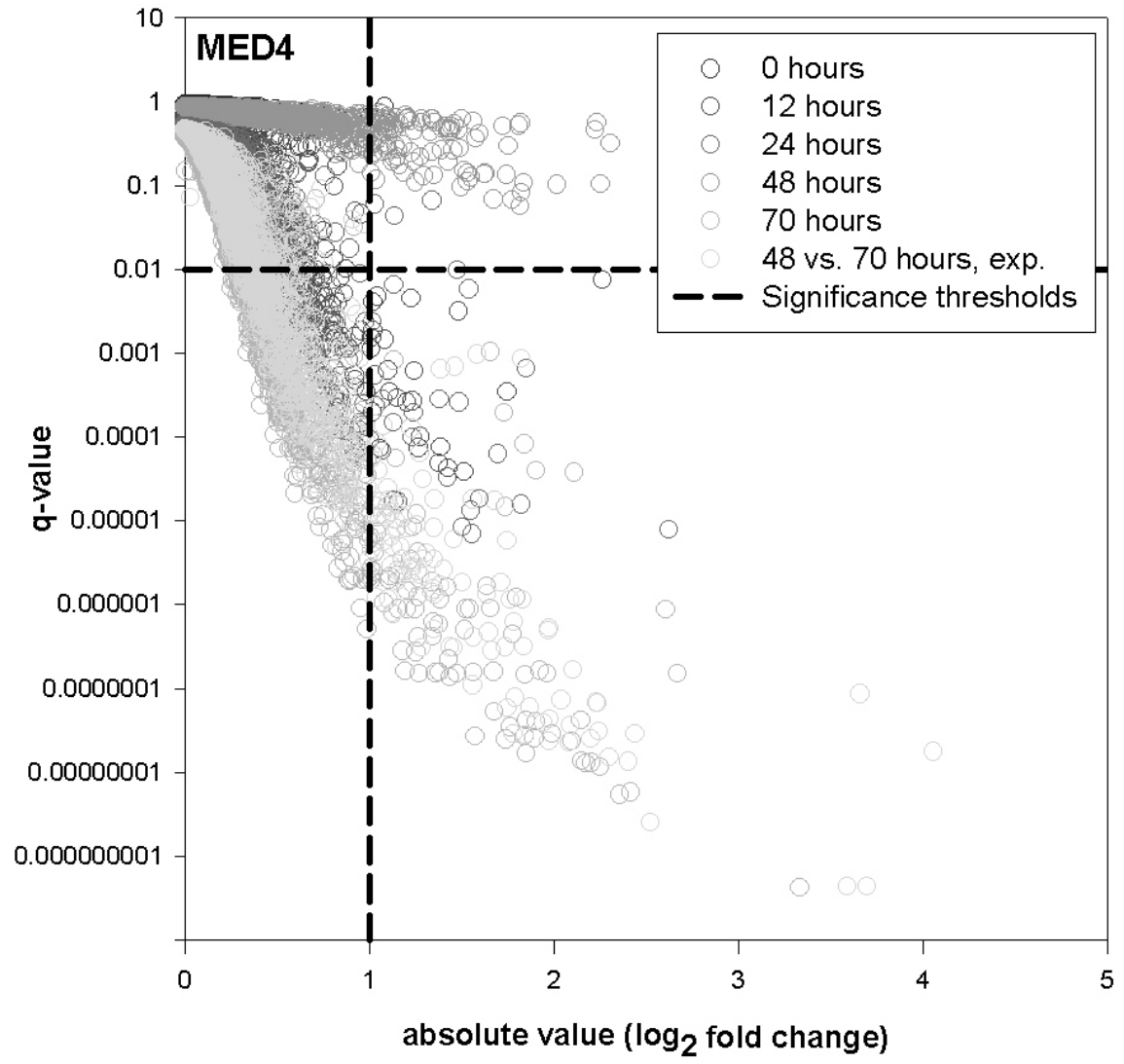

Figure 1: MED4 differentially expressed genes in response to iron. Comparison of the q-values (false discovery rate) and fold change (as absolute value of $\log _{2}$ (fold change) ) for each gene in each comparison of MED4 iron starvation or recovery. Genes that are located in the shaded area are considered differentially expressed genes and have q-values less than 0.01 (horizontal dotted line) and absolute value $\log _{2}$ (fold change) greater than 1 (vertical dotted line). 


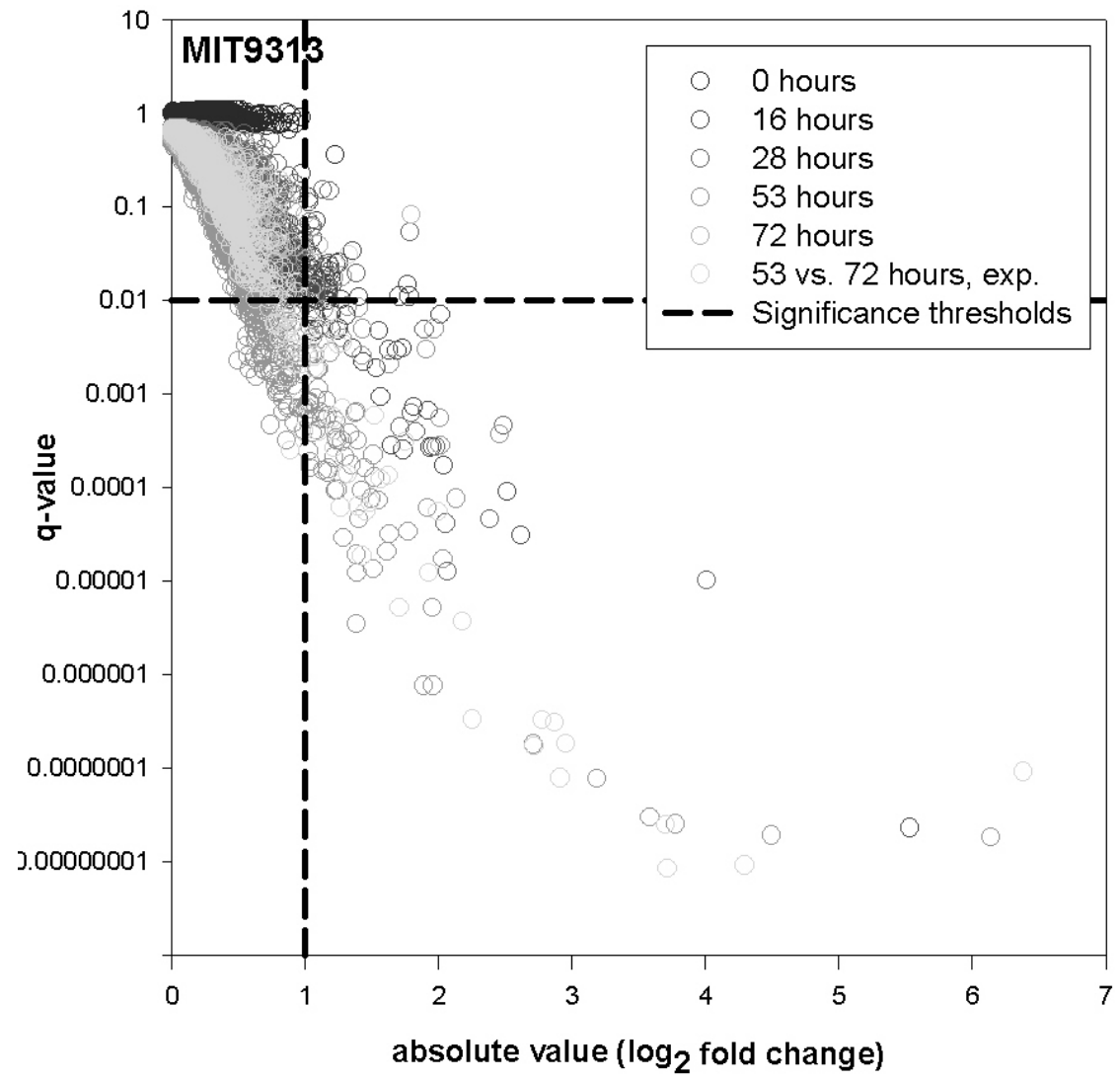

Figure 2: MIT9313 differentially expressed genes in response to iron.

Comparison of the q-values (false discovery rate) and fold change (as absolute value of $\log _{2}$ (fold change)) for each gene in each comparison of MIT9313 iron starvation or recovery. Genes that are located in the shaded area are considered differentially expressed genes and have q-values less than 0.01 (horizontal dotted line) and absolute value $\log _{2}$ (fold change) greater than 1 (vertical dotted line). 


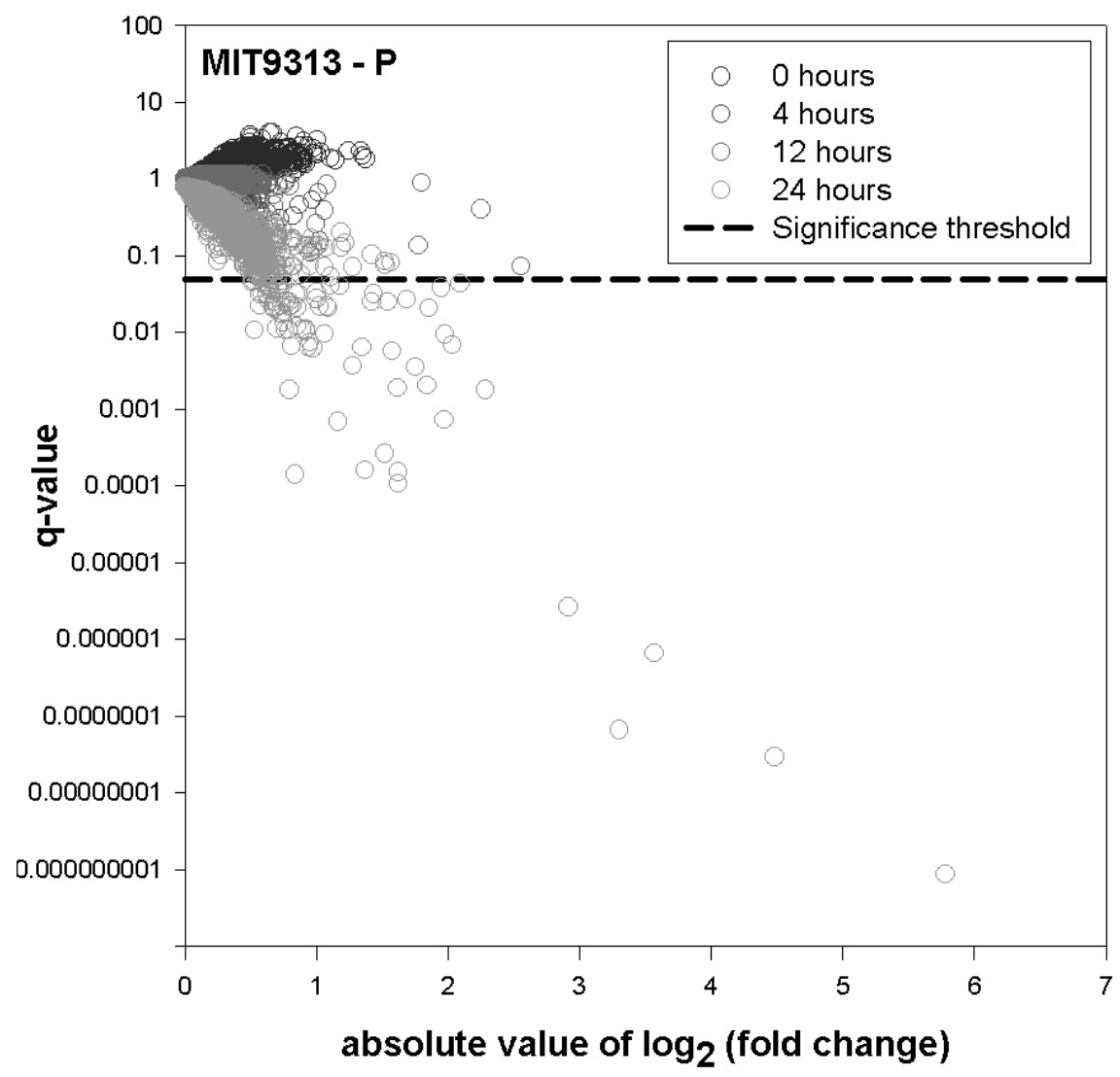

Figure 3: MIT9313 differentially expressed genes in response to phosphate starvation. Comparison of the q-values (false discovery rate) and fold change (as absolute value of $\log _{2}$ (fold change)) for each gene in each comparison of MIT9313 iron starvation or recovery. Genes that are located in the shaded area are considered differentially expressed genes and have q-values less than 0.05 (horizontal dotted line). This significance threshold is the same as for the experiment when it was first published in Martiny and Coleman et al. 2006. 


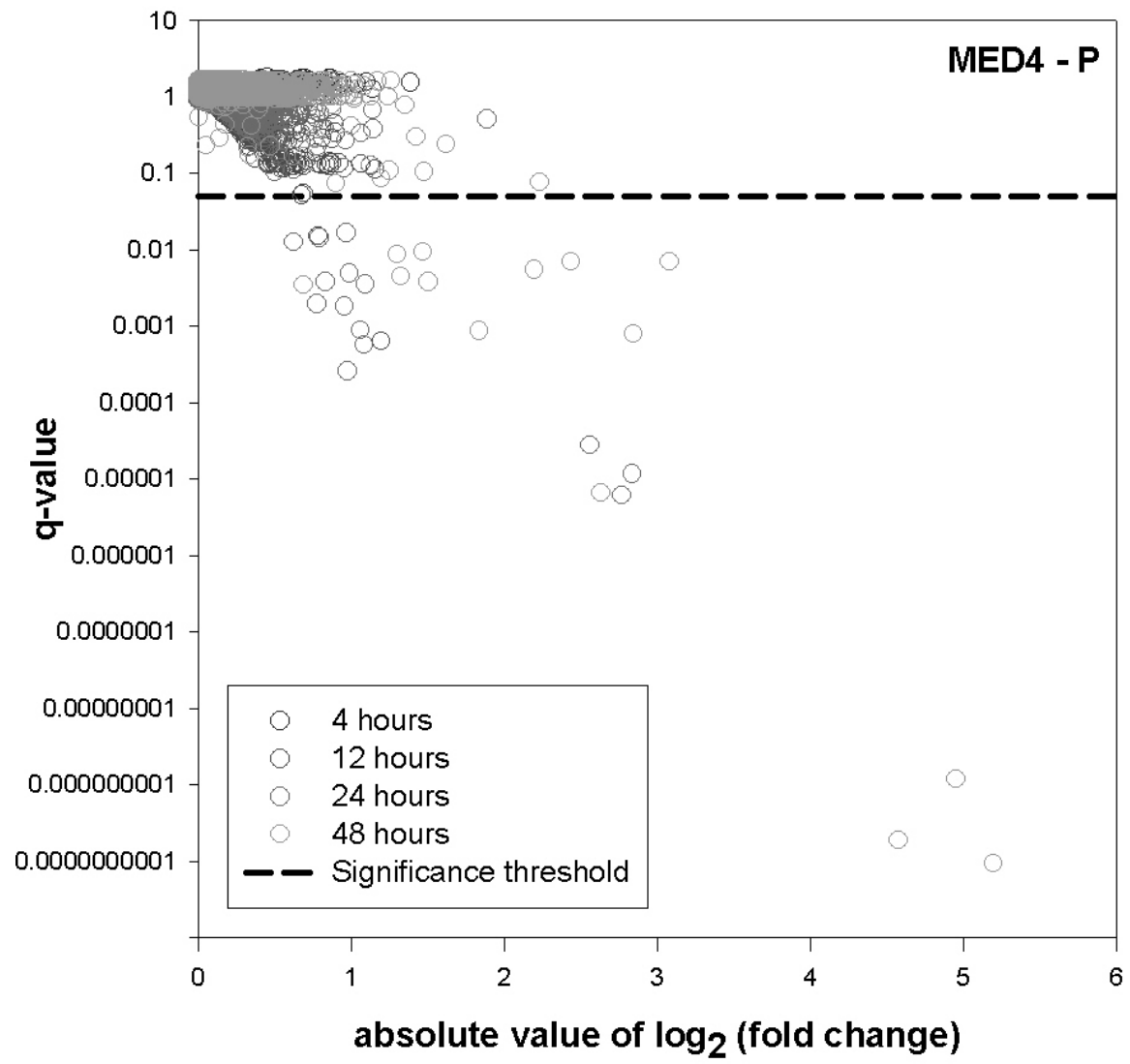

Figure 4: MED4 differentially expressed genes in response to phosphate starvation. Comparison of the q-values (false discovery rate) and fold change (as absolute value of $\log _{2}$ (fold change)) for each gene in each comparison of MED4 iron starvation or recovery. Genes that are located in the shaded area are considered differentially expressed genes and have q-values less than 0.05 (horizontal dotted line). This significance threshold is the same as for the experiment when it was first published in Martiny and Coleman et al. 2006. 


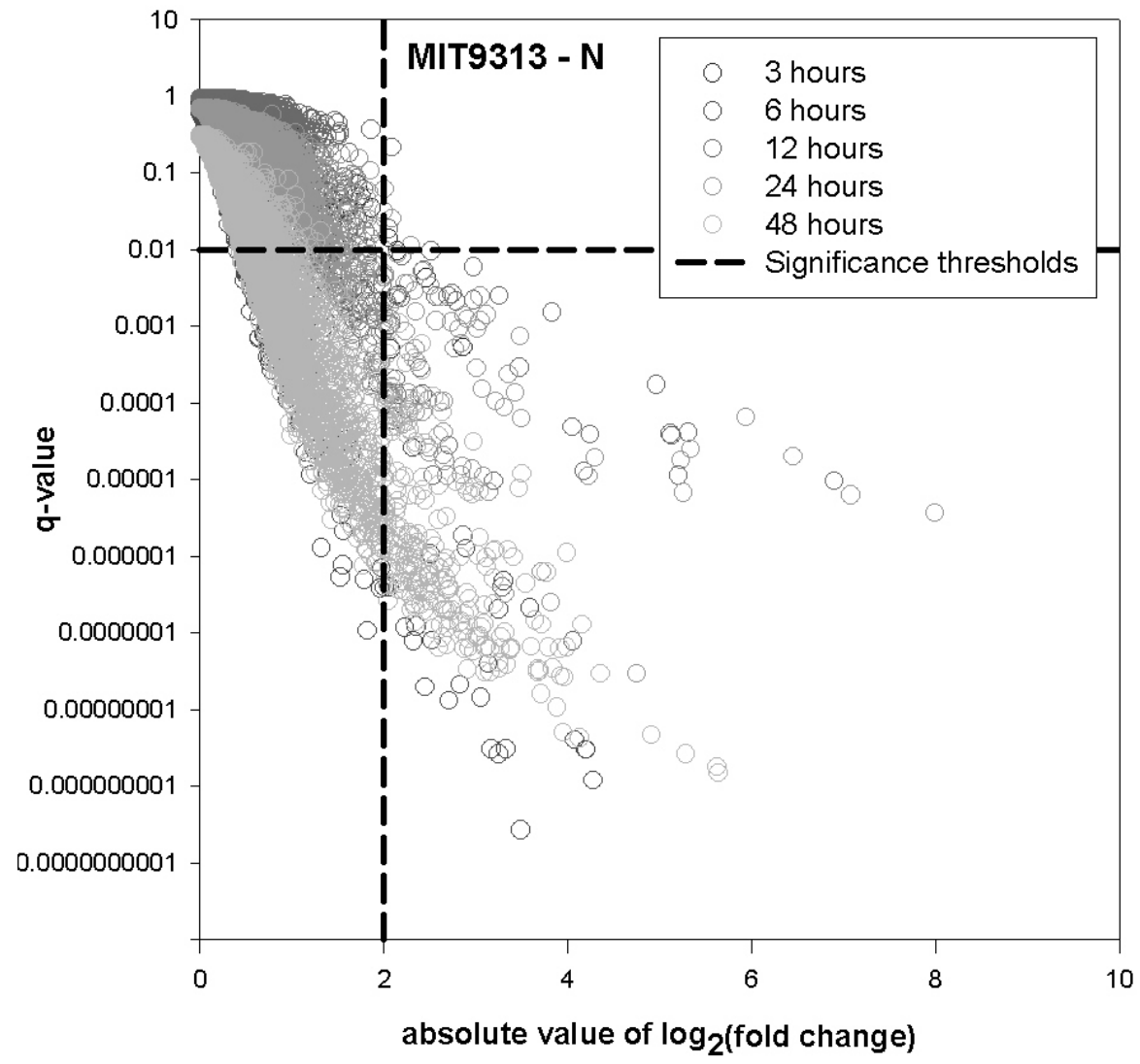

Figure 5: MIT9313 differentially expressed genes in response to nitrogen starvation. Comparison of the q-values (false discovery rate) and fold change (as absolute value of $\log _{2}$ (fold change)) for each gene in each comparison of MIT9313 iron starvation or recovery. Genes that are located in the shaded area are considered differentially expressed genes and have q-values less than 0.01 (horizontal dotted line) and $\log _{2}$ (fold change) greater than 2 or less than -2 . The fold change significance threshold is higher than the what was used in the published study of Tolonen et al. 2006. 


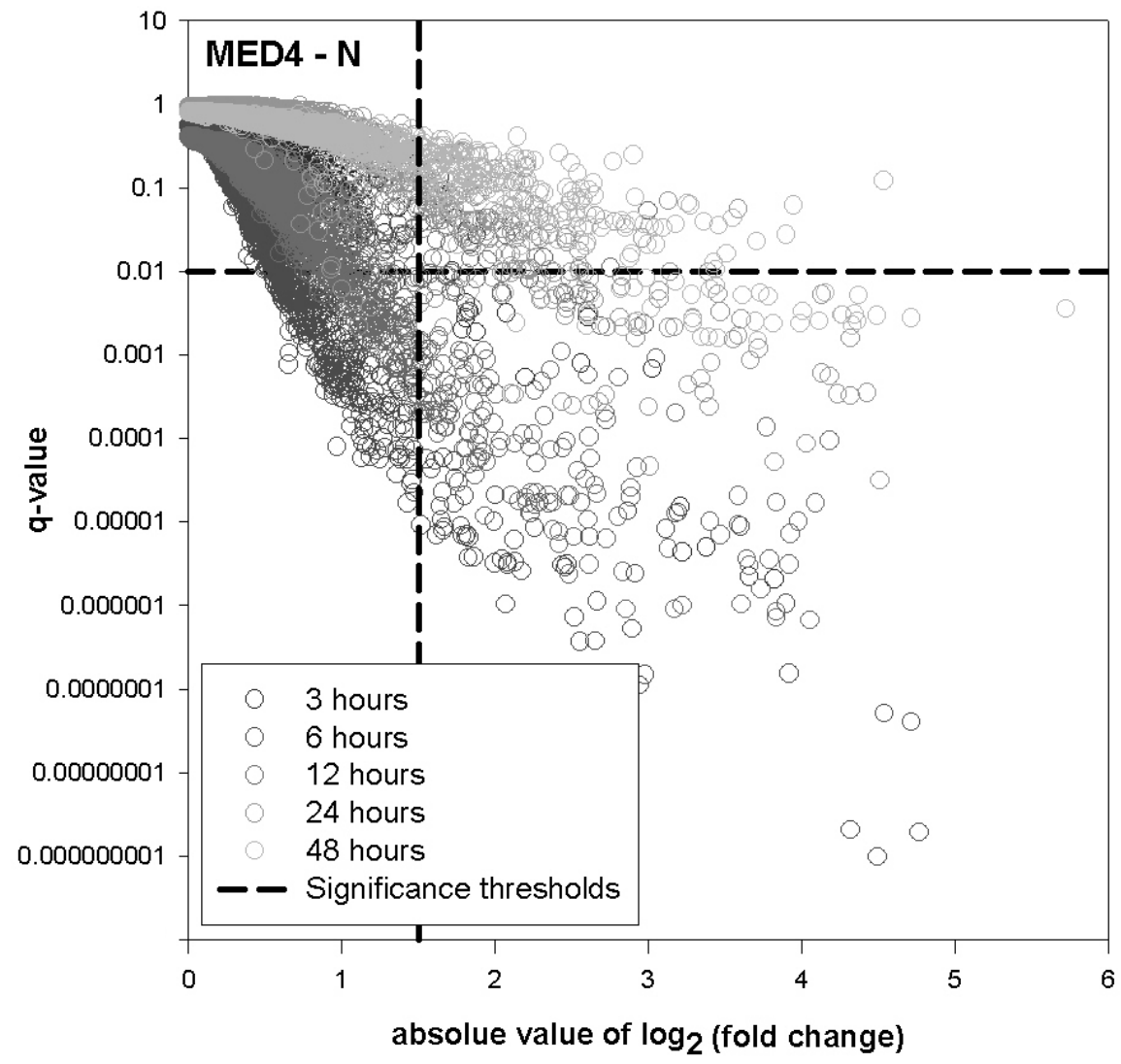

Figure 6: MED4 differentially expressed genes in response to nitrogen starvation. Comparison of the q-values (false discovery rate) and fold change (as absolute value of $\log _{2}$ (fold change)) for each gene in each comparison of MED4 iron starvation or recovery. Genes that are located in the shaded area are considered differentially expressed genes and have q-values less than 0.01 (horizontal dotted line) and $\log _{2}$ (fold change) greater than 1.5 or less than -1.5 . The fold change significance threshold is higher than the what was used in the published study of Tolonen et al. 2006. 


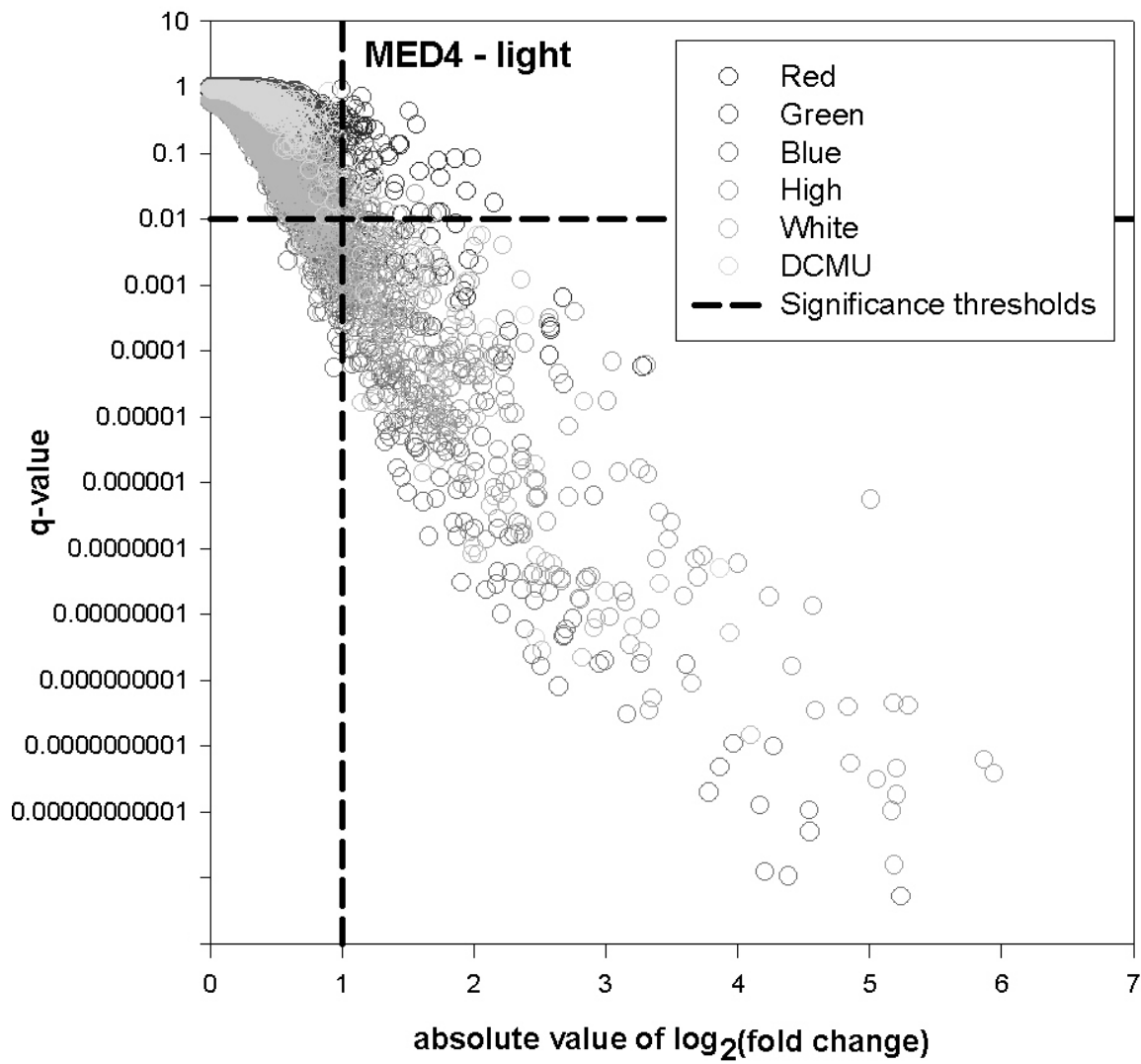

Figure 7: MED4 differentially expressed genes in response to light quality and quantity. Comparison of the q-values (false discovery rate) and fold change (as absolute value of $\log _{2}$ (fold change)) for each gene in each comparison of MED4 iron starvation or recovery. Genes that are located in the shaded area are considered differentially expressed genes and have q-values less than 0.01 (horizontal dotted line) and $\log _{2}$ (fold change) greater than 1 or less than -1 . The fold change significance threshold is higher than the what was used in the published study of Steglich et al. 2006. 


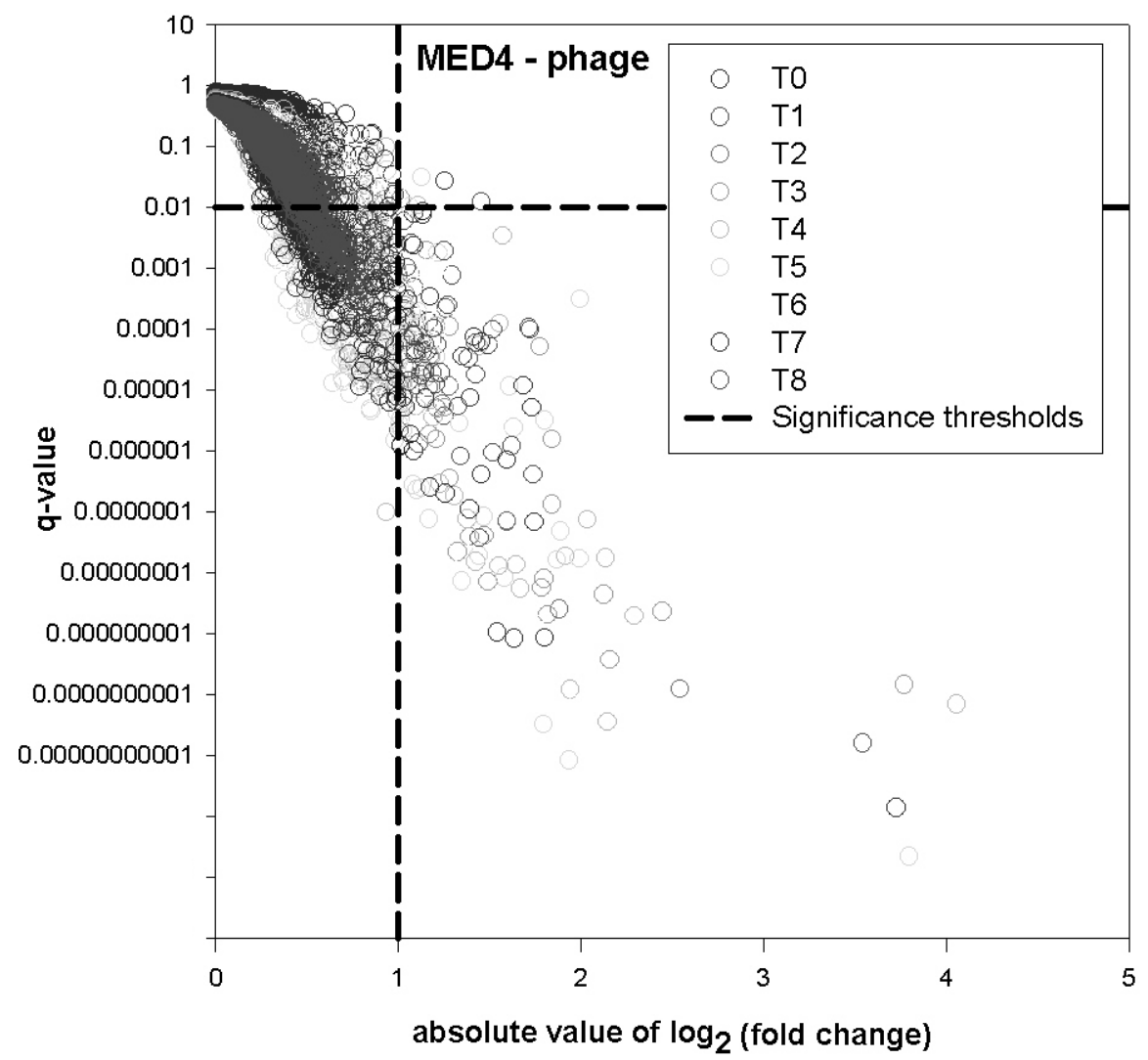

Figure 8: MED4 differentially expressed genes in response to phage infection. Comparison of the q-values (false discovery rate) and fold change (as absolute value of $\log _{2}$ (fold change)) for each gene in each comparison of MED4 iron starvation or recovery. Genes that are located in the shaded area are considered differentially expressed genes and have q-values less than 0.01 (horizontal dotted line) and $\log _{2}$ (fold change) greater than 1 or less than -1 . The fold change significance threshold is higher than the what was used in the published study of Lindell et al. 2007. 


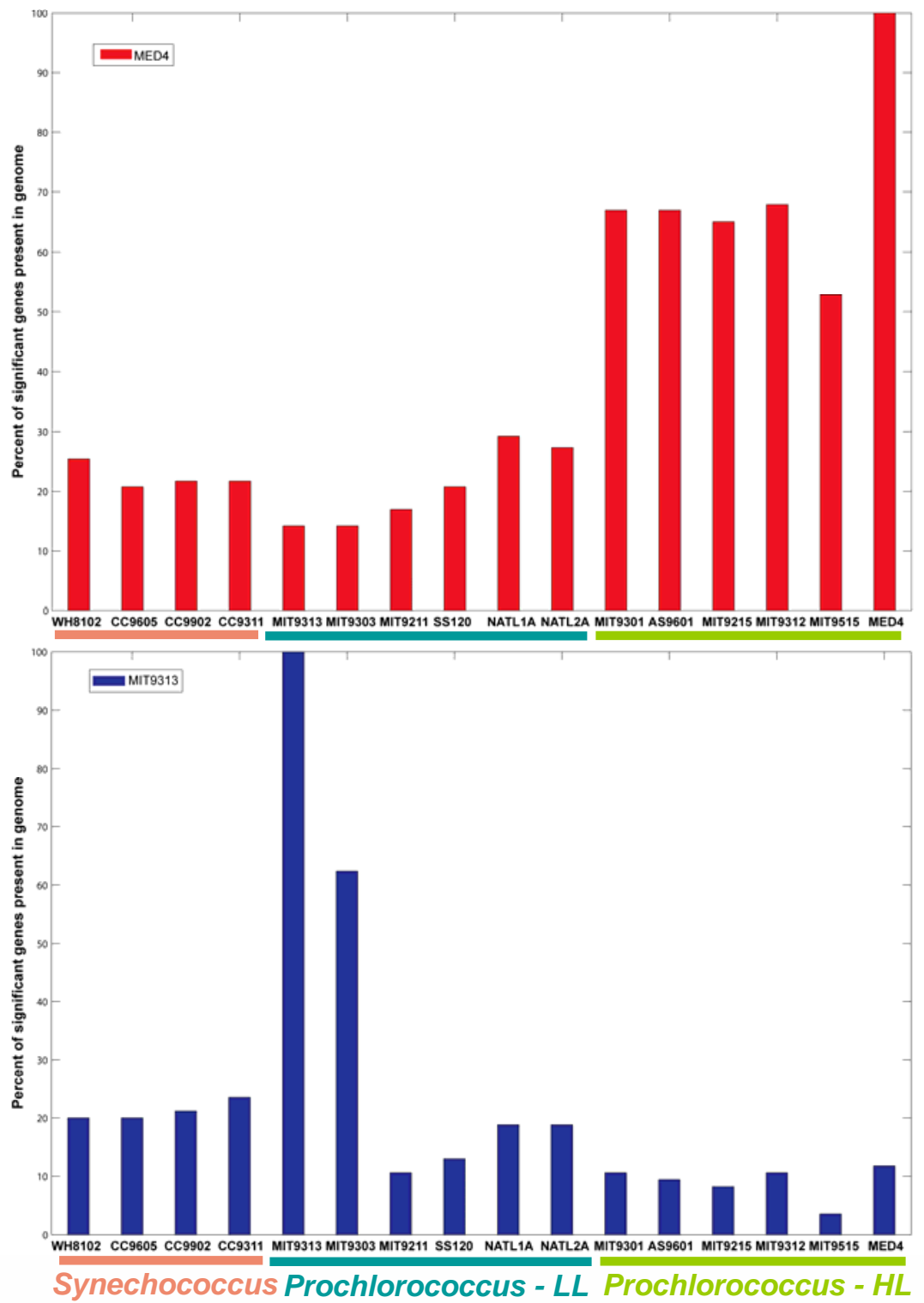

Figure 9: Distribution of MED4 and MIT9313 flexible genes that are significantly differentially expressed in response to $\mathrm{N}$ starvation (Tolonen et al. 2006) among the other sequenced Prochlorococcus isolates and four Synechococcus isolates that were examined in Kettler et al. 2007. Genomes are organized along the $\mathrm{x}$-axis according to phylogeny and the $\mathrm{y}$-axis represents the percentage of either MED4 or MIT9313 iron-responsive genes that are present in each genome. 

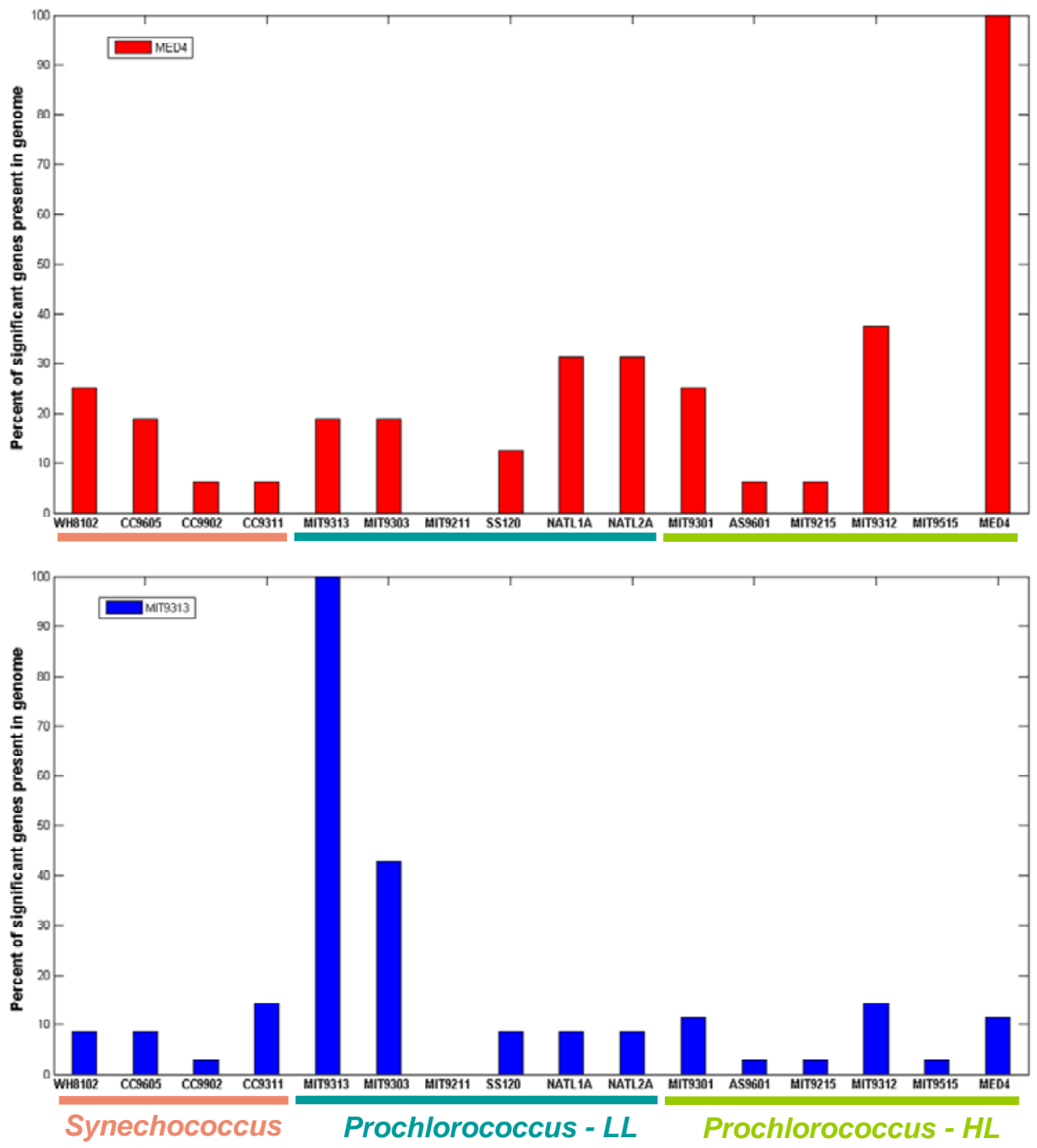

Figure 10: Distribution of MED4 and MIT9313 flexible genes that are significantly differentially expressed in response to P starvation (Martiny and Coleman et al. 2006) among the other sequenced Prochlorococcus isolates and four Synechococcus isolates that were examined in Kettler et al. 2007. Genomes are organized along the $\mathrm{x}$-axis according to phylogeny and the $\mathrm{y}$-axis represents the percentage of either MED4 or MIT9313 iron-responsive genes that are present in each genome. 


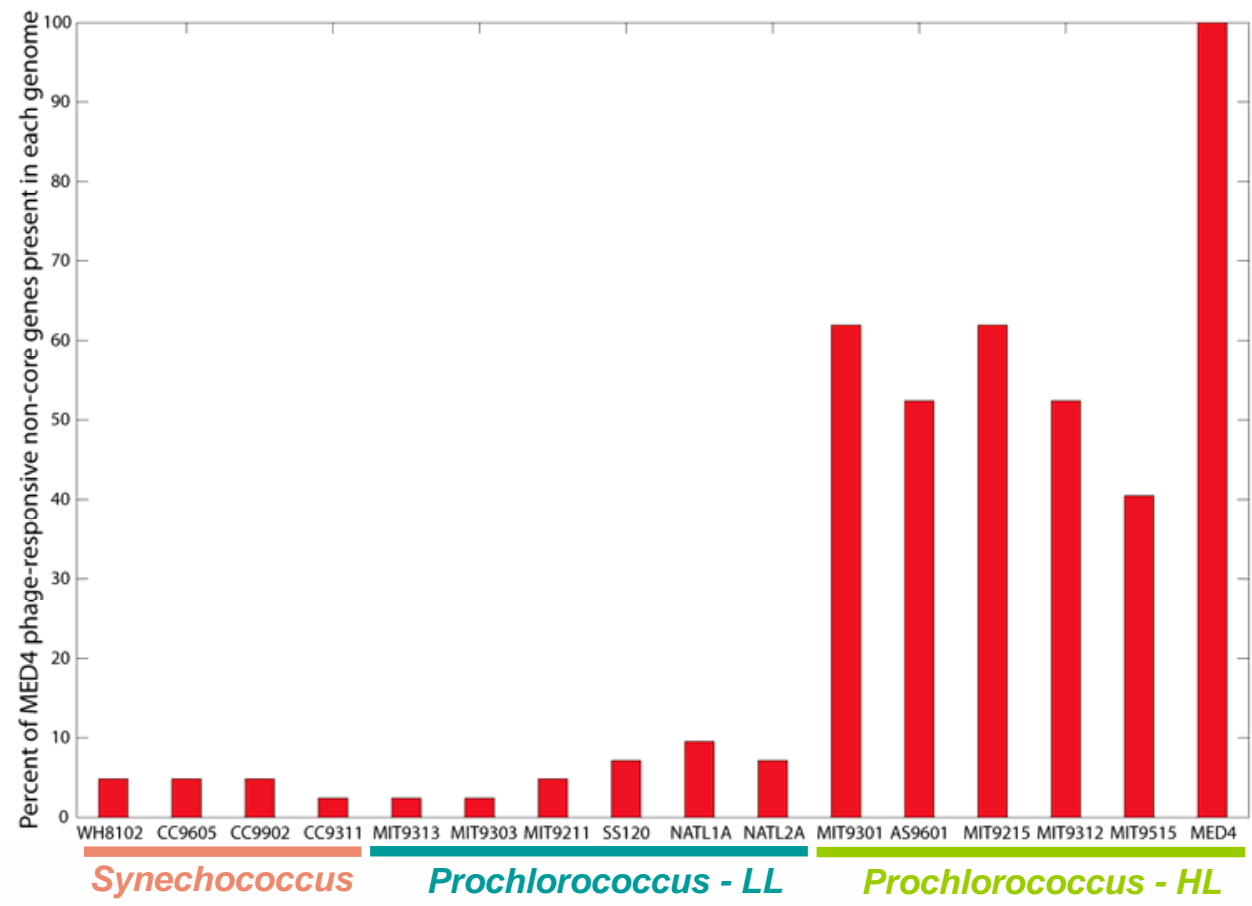

Figure 11: Distribution of MED4 flexible genes that are significantly differentially expressed in response to phage infection (Lindell et al. 2007) among the other sequenced Prochlorococcus isolates and four Synechococcus isolates that were examined in Kettler et al. 2007. Genomes are organized along the $\mathrm{x}$-axis according to phylogeny and the y-axis represents the percentage of MED4 ironresponsive genes that are present in each genome. 


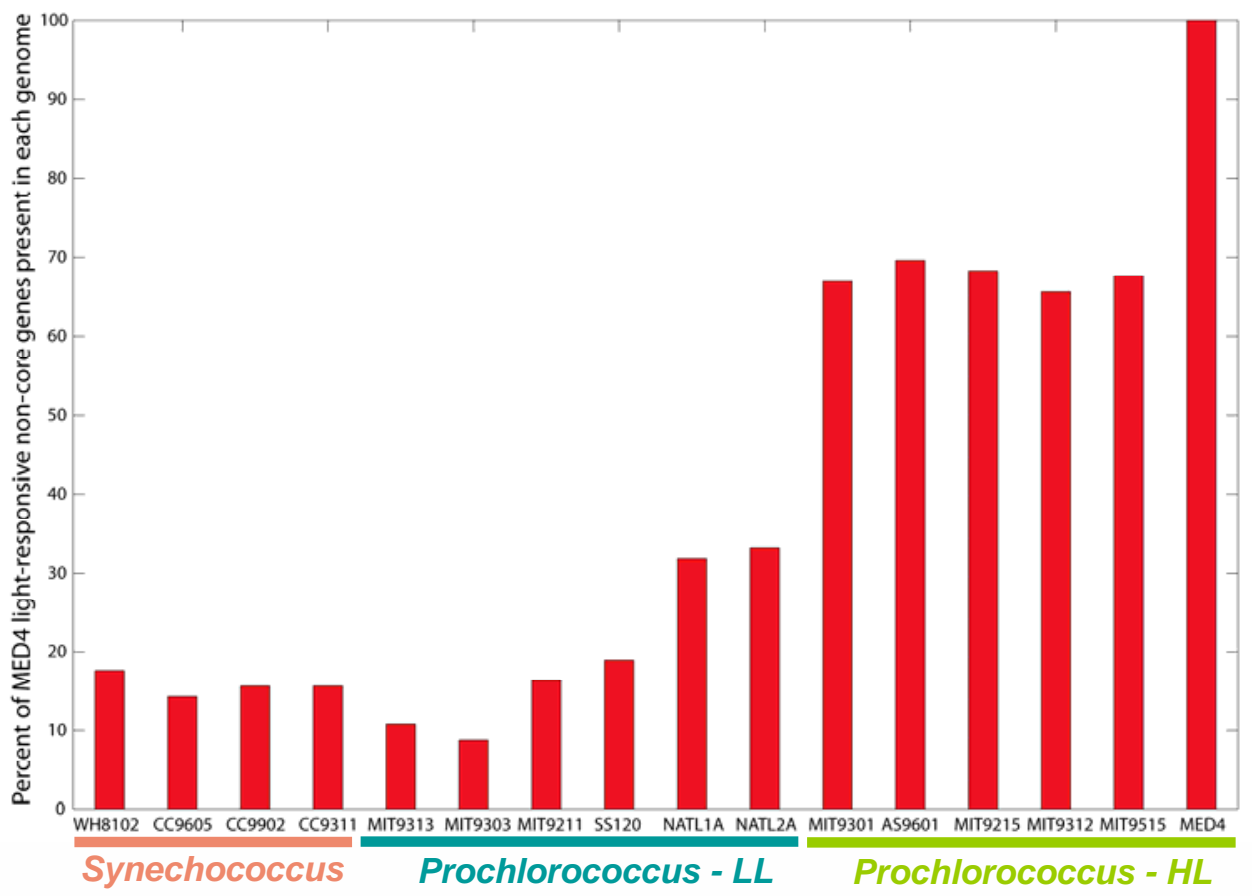

Figure 12: Distribution of MED4 flexible genes that are significantly differentially expressed in response to shifts in light quality and quantity (Steglich et al. 2006) among the other sequenced Prochlorococcus isolates and four Synechococcus isolates that were examined in Kettler et al. 2007. Genomes are organized along the $\mathrm{x}$-axis according to phylogeny and the $\mathrm{y}$-axis represents the percentage of MED4 iron-responsive genes that are present in each genome. 


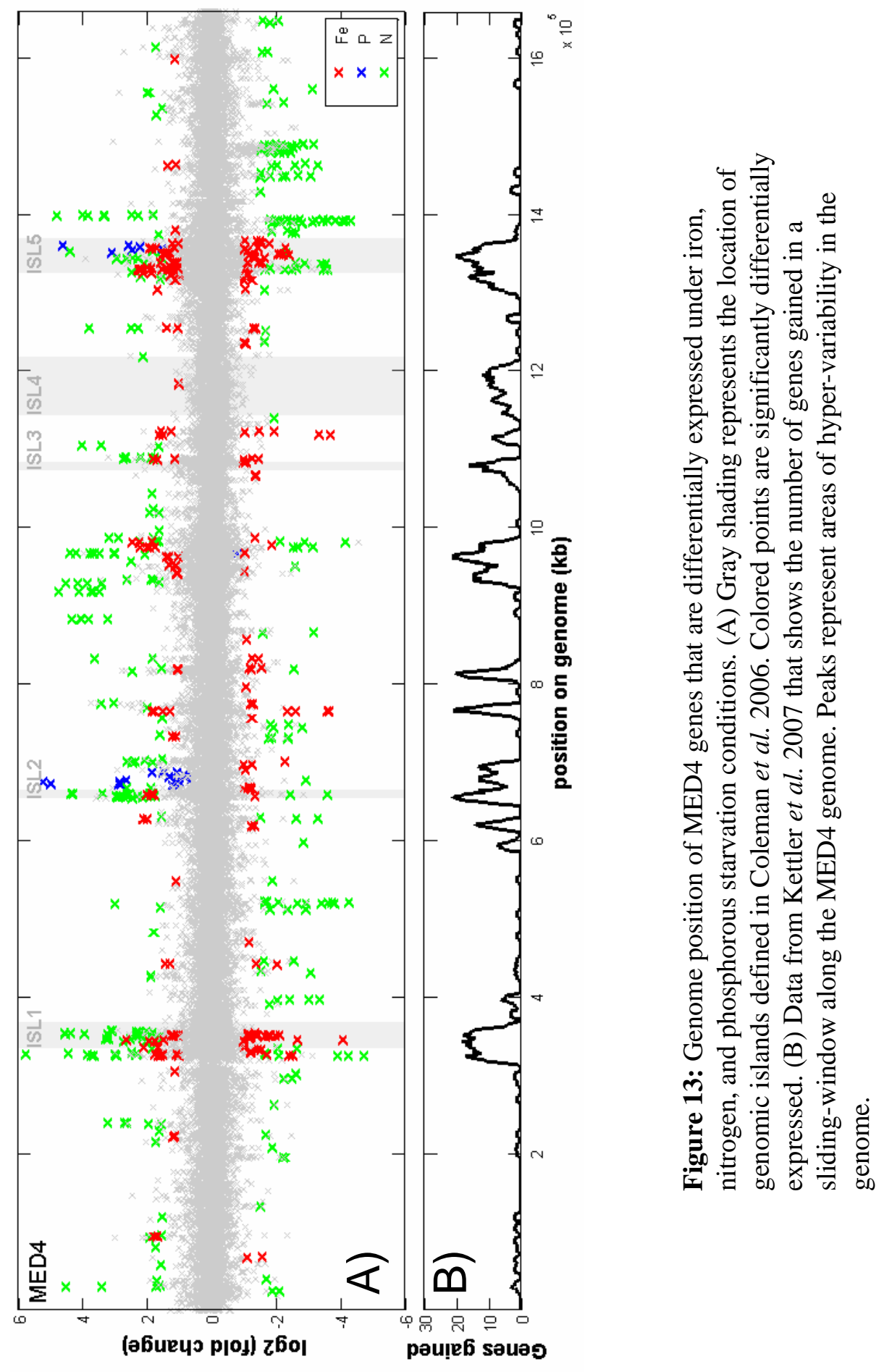




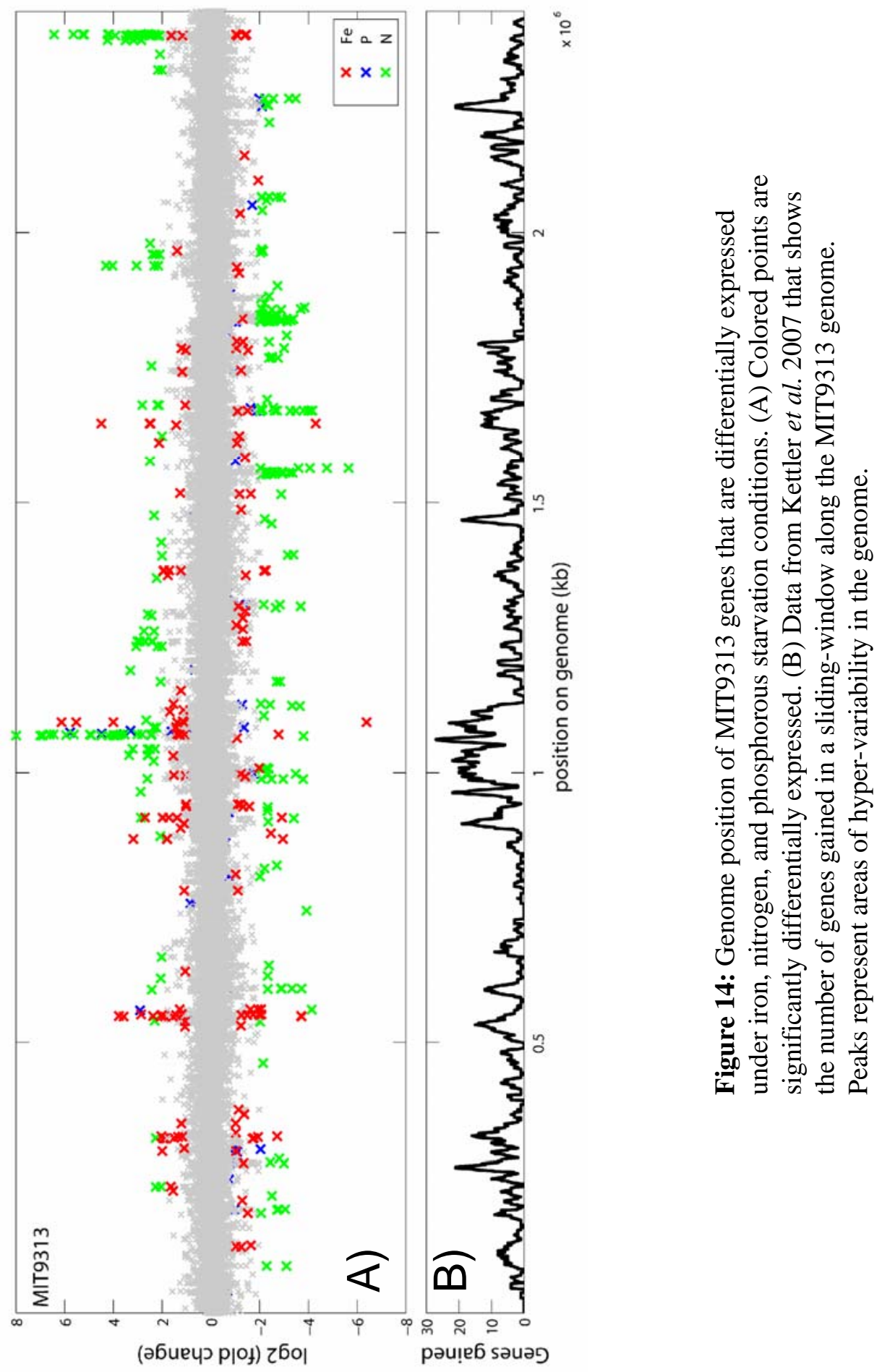




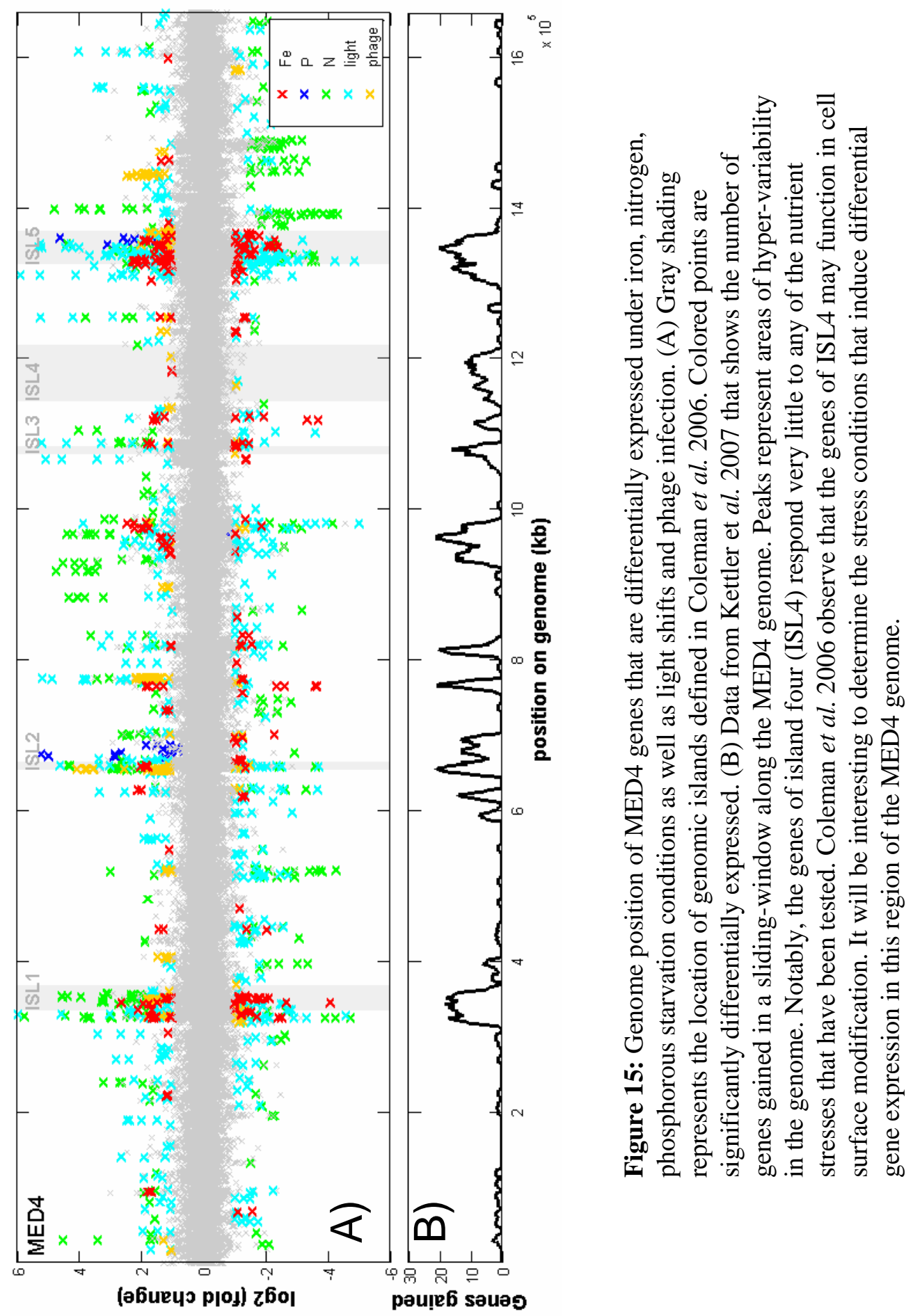



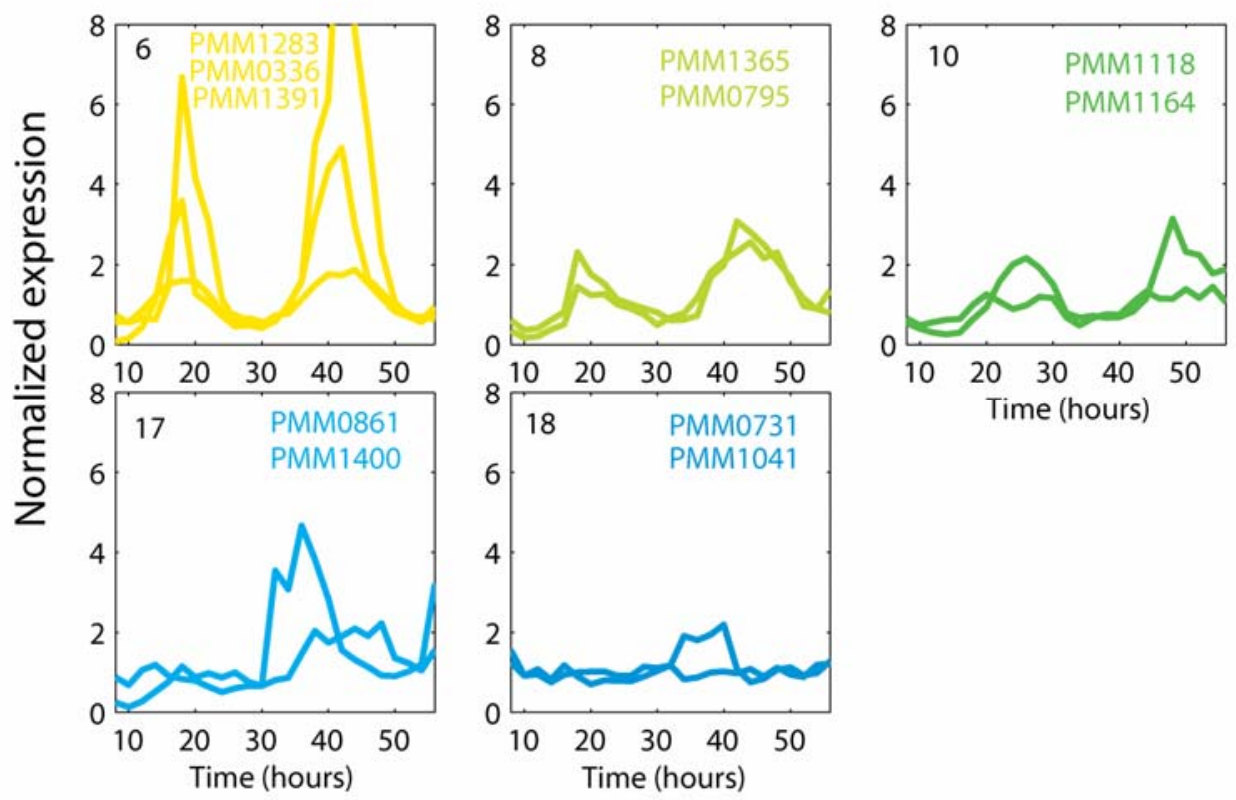

Figure 16: The diel expression and cluster-membership of genes that are significantly differentially expressed with iron starvation and cluster with afuA (or, idiA) that is up-regulated in MED4 during iron starvation. Numbers in the top left corners identify the cluster the genes belong to in the diel experiment (Zinser et al. 2009). Clusters 1-16 are considered to be periodically expressed with light availability. Cluster 17 contains genes that are a-periodic. Cluster 18 contains genes that are not expressed in the experiment. 

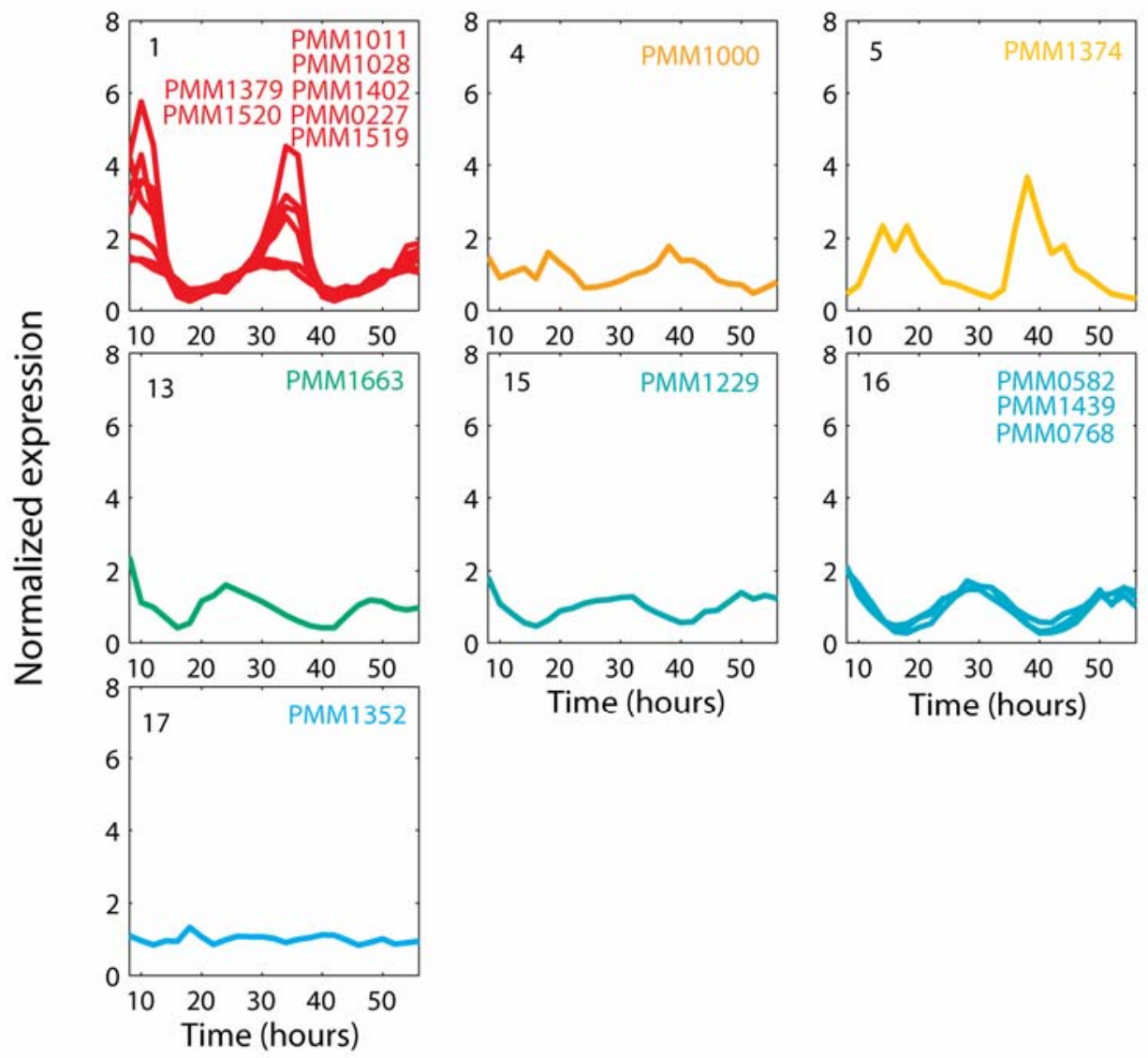

Figure 17: The diel expression and cluster-membership of genes that are significantly differentially expressed with iron starvation and cluster with petF (ferredoxin) that is down-regulated in MED4 during iron starvation. Numbers in the top left corners identify the cluster the genes belong to in the diel experiment (Zinser et al. 2009). Clusters 1-16 are considered to be periodically expressed with light availability. Cluster 17 contains genes that are a-periodic. Cluster 18 contains genes that are not expressed in the experiment. 
Figure 18: The diel expression and cluster-membership of genes that are significantly differentially expressed with iron starvation and cluster with isiB (flavodoxin) that is up-regulated in MED4 during iron starvation. Numbers in the top left corners identify the cluster the genes belong to in the diel experiment (Zinser et al. 2009). Clusters 1-16 are considered to be periodically expressed with light availability. Cluster 17 contains genes that are a-periodic. Cluster 18 contains genes that are not expressed in the experiment. 

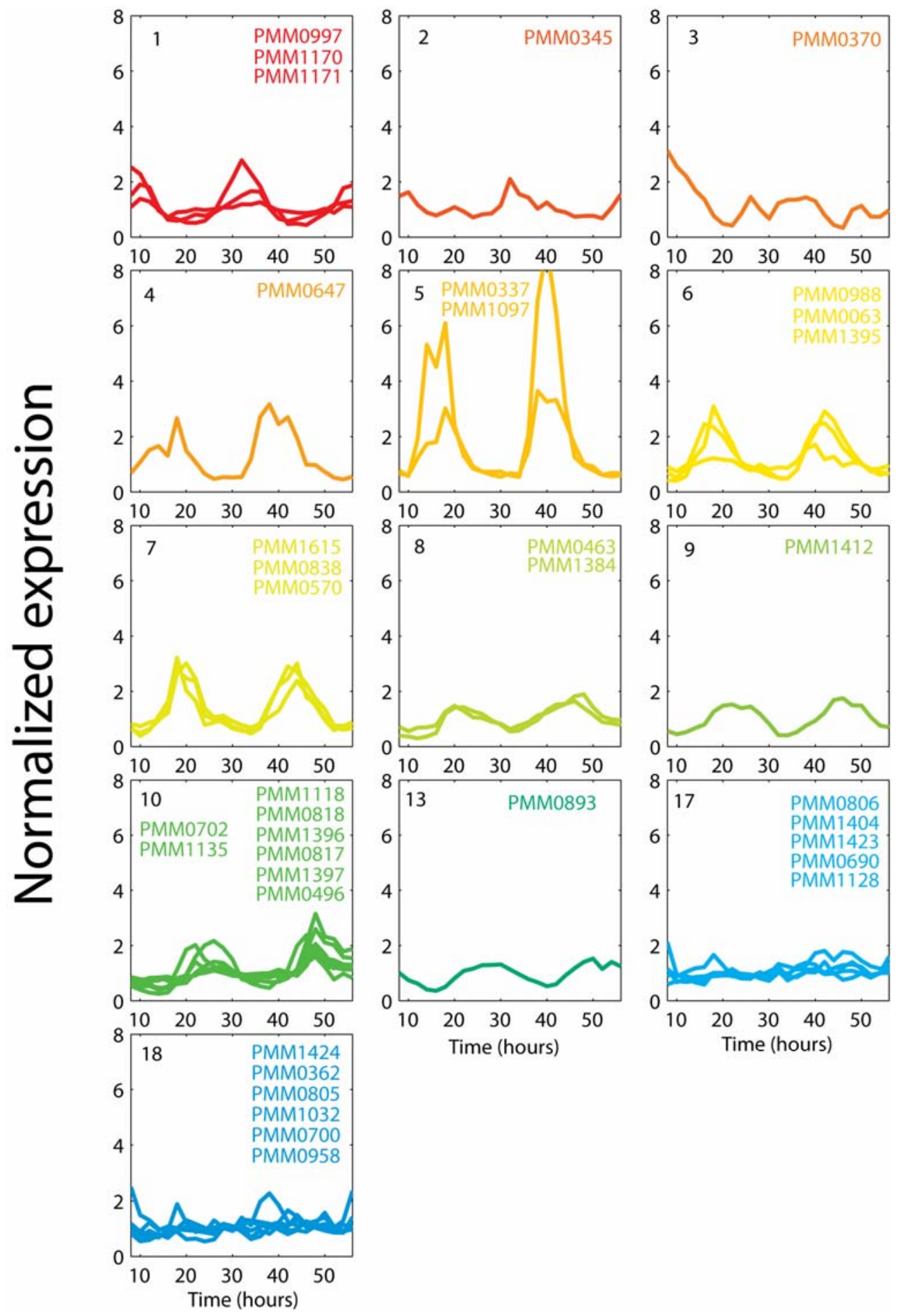

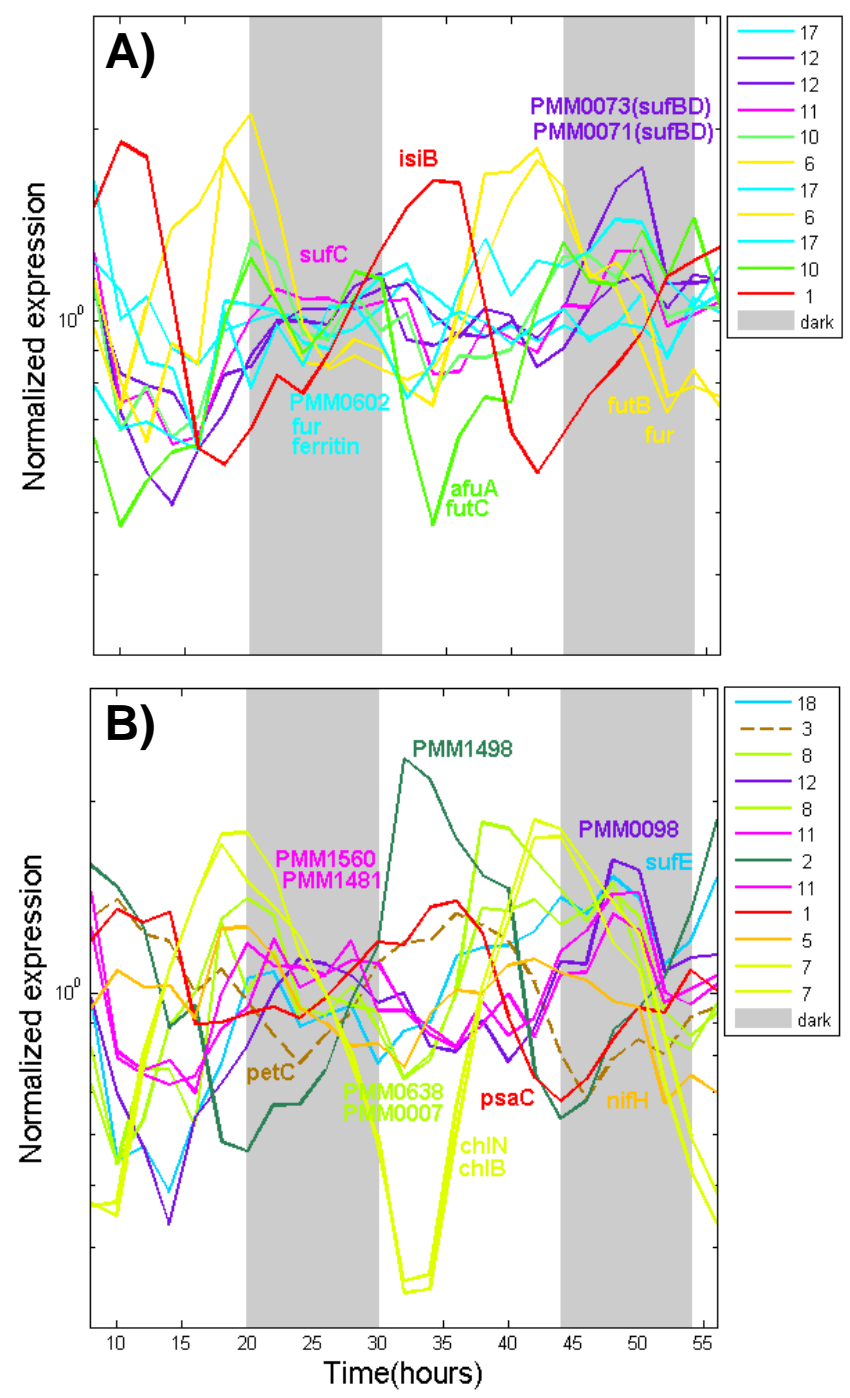

Figure 19: MED4 diel expression of Prochlorococcus core genes that are predicted to be related to iron. Expression data is published in Zinser et al. 2009. (A) Genes predicted to be involved in iron transport, storage, or regulation. The iron stress indicator gene, $i s i B$, is also presented in this panel. (B) Genes that are predicted to contain Fe-S clusters. Genes are colored by their cluster membership in the diel expression experiment. Clusters 1-16 contain genes that are periodic over the diel cycle. Cluster 17 contains a-periodic genes. Cluster 18 contains genes that appear not to be expressed above a background threshold defined in Zinser et al. 2009. Dark shading indicates periods of darkness during the two diel cycles that are presented here. Continued next page. 


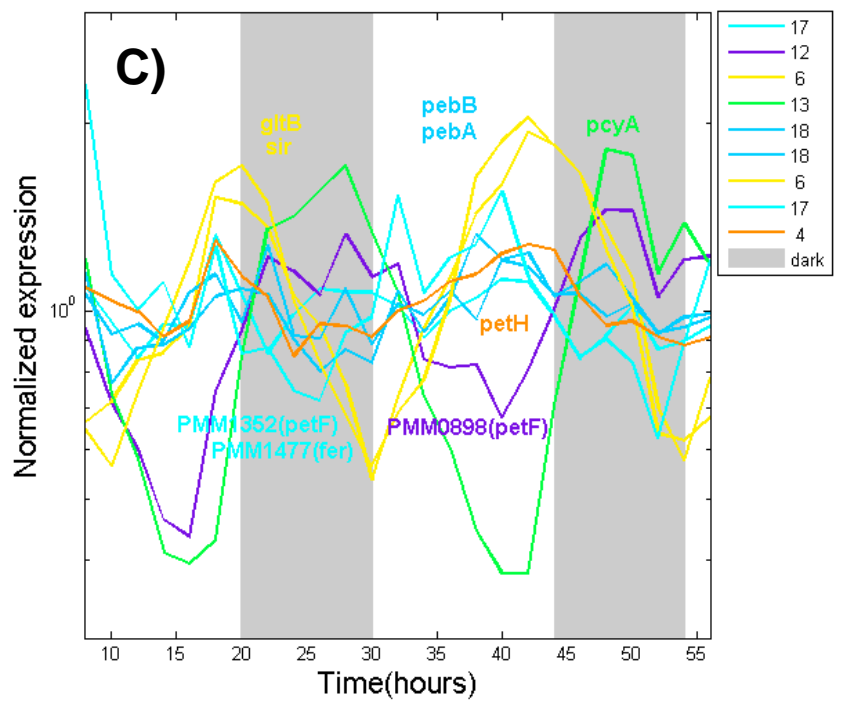

Figure 19, continued: (C) Genes that are predicted to encode ferredoxin proteins or ferredoxin-dependent proteins. Genes are colored by their cluster membership in the diel expression experiment. Clusters 1-16 contain genes that are periodic over the diel cycle. Cluster 17 contains a-periodic genes. Cluster 18 contains genes that appear not to be expressed above a background threshold defined in Zinser et al. 2009. Dark shading indicates periods of darkness during the two diel cycles that are presented here. 


\section{APPENDIX C}

Reliability of expired custom M4-9313 Affymetrix microarray chips 


\section{Introduction and Motivation}

In this experiment, the reliability of expired custom M4-9313 Affymetrix arrays is tested to determine if samples hybridized to expired arrays could be compared to samples hybridized to non-expired arrays from a new batch. The motivation for this comparison stems from the situation in January 2008 when the Chisholm Lab wanted to prevent wasting the many expired arrays that were on hand, if possible.

\section{Methods}

Two samples of MIT9313 RNA (extraction and amplification described in Chapter 3 methods) were submitted to the Harvard Biopolymers Facility (Boston, MA) for hybridization to the custom M4-9313 Affymetrix microarray. Biological replicates were hybridized to two separate expired microarrays. An identical sample of the first biological replicate was hybridized to an array from a fresh batch of non-expired arrays. The raw expression values for each probeset were plotted on an x-equals-y plot to compare the expression pattern of the three hybridized samples. Comparisons were conducted without replication because of the expense of using and processing each microarray.

\section{Results}

Figure 1 displays a comparison of the expression signal for each probeset between the same sample (replicate A) hybridized to the expired array and non-expired array. The black line represents the best fit line to the data. Probesets are most similar between the biological replicates that are both hybridized to expired arrays (Figure 1b). This result suggests that use of this batch of recently expired arrays will yield robust expression data. Yet, comparing the same sample hybridized to both an expired array and a non-expired array (Figure 1a), we detect a poorer fit to a 1:1 line than the biological replicates that were both hybridized to arrays from the same batch. This experiment suggests that comparison of samples between different batches of arrays yields less robust results than the comparison between samples that are hybridized to arrays of the same batch. It is unclear whether the difference in hybridization is a result of degradation of the printed oligonucleotides on the array after their expiration date, or, if different batches of arrays differ slightly independently of the time past their expiration. 


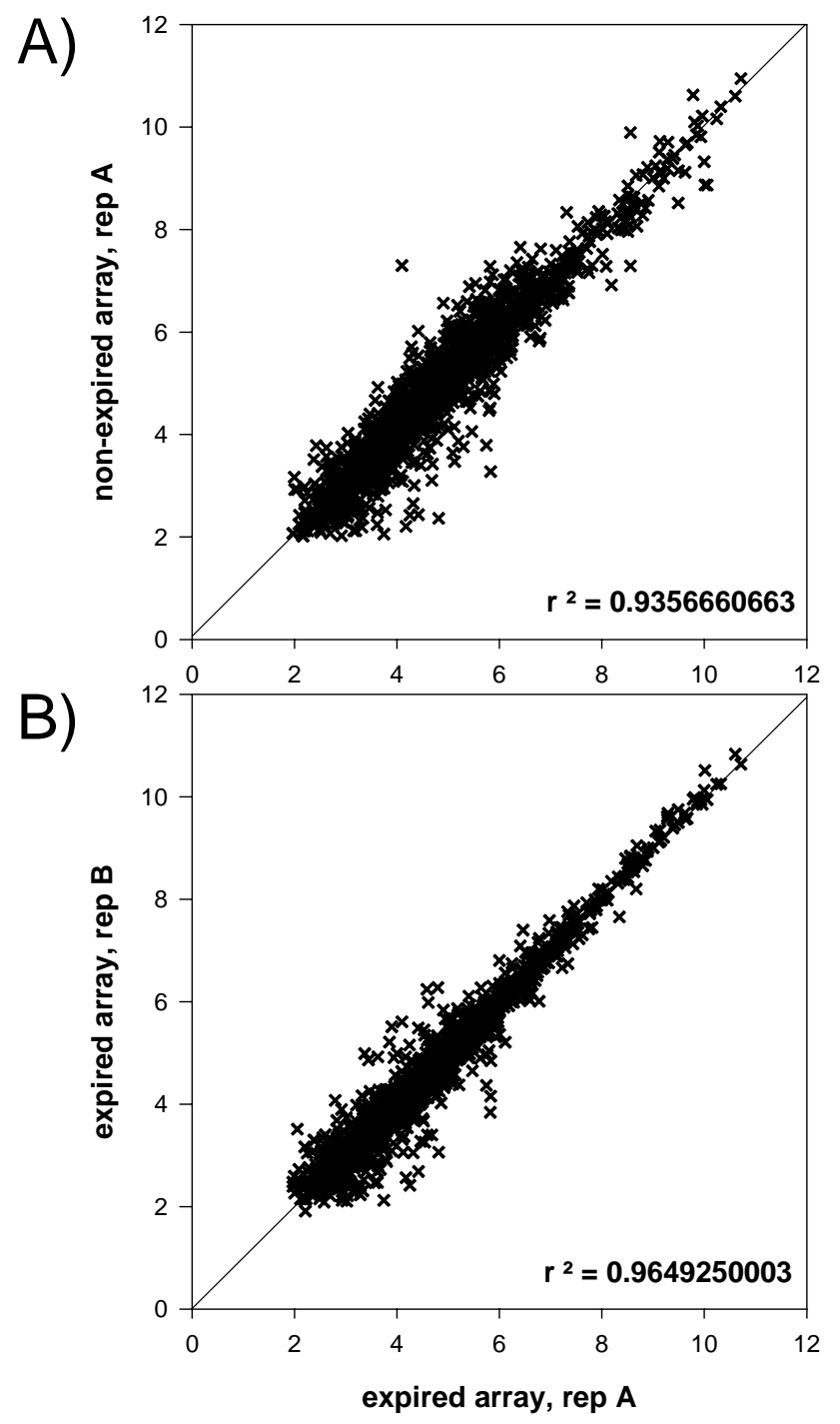

Figure 1: Reliability of expired custom M4-9313 Affymetrix Arrays. (A) Relationship between gene expression of replicate A hybridized to an expired array (x-axis) and a non-expired array (y-axis). (B) Relationship between biological duplicates (replicate A and B) both hybridized to expired arrays. Results indicate that different batches of arrays yield different patterns of expression. It would be most important to make sure samples are hybridized to the same batch of arrays, even if expired, if they are to be directly compared. 


\section{APPENDIX D}

The effect of centrifugation on Prochlorococcus global gene expression 


\section{Introduction and Motivation}

Many of the published Prochlorococcus microarray experiments involve centrifuging cultures in order to initiate nutrient starvation and to sample cultures for RNA (Martiny et al, 2006; Tolonen et al, 2006). Though we presumed that gene expression in response to centrifugation would be the same in experimental and control conditions and thus controlled for, as an additional control we asked: How does the global gene expression of Prochlorococcus respond to centrifugation?

\section{Methods}

MED4 was grown in duplicate to mid-log phase in media with 10-fold more EDTA than PRO99. The duplicate control RNA samples were collected by centrifuging $300 \mathrm{ml}$ of each culture by centrifugation for 10 minutes at $8500 \mathrm{rpm}$, re-suspension in RNA buffer, and flash freezing (Chapter 3). For the experimental samples, the remaining twp cultures were treated as in Chapter 3 - Methods, for the initial set-up of the experiment. These are the steps that would be taken to initiate nutrient stress as in Martiny and Coleman et al. 2006, Tolonen et al. 2006, and the iron deprivation experiments in Chapter 3 of this thesis. RNA samples were collected immediately from the large volumes by centrifugation for 15 minutes at $8500 \mathrm{rpm}$, re-suspension in RNA buffer, and flash frozen. RNA was extracted from samples and hybridized to Affymetrix microarrays and normalized as in Chapter 3. Significantly differentially expressed genes had a qvalue less than 0.05 and fold-change ( $\log _{2}$ experimental treatment/control treatment) greater than 1 or less than -1 . We also tracked the expression of genes with q-value less than 0.05 that did not meet the fold-change threshold (Figure 1).

\section{Results}

Nine genes in MED4 were significantly differentially expressed in response to centrifugation. Figure 1 displays the results of comparing of the mean expression signal for each gene on the array with each treatment. It is important to note that the fold change between experimental and control treatments was very near to the significance threshold for all genes suggesting a minimal response to centrifugation and the other steps taken to collect the samples. The small non-coding RNA, Yfr12, was the only gene down-regulated in response to centrifugation. Among the genes up-regulated in response to centrifugation are: hli5 (and PMM1404), the 30S ribosomal protein S4 (PMM0268), a putative RNA polymerase sigma factor, 
sigA (PMM0496), a Type II alternative RNA polymerase sigma factor (PMM1629), the RNAbinding region RNP-1 (PMM0142), non-coding RNA Y fr6, and one conserved hypothetical protein (PMED4_08021). 


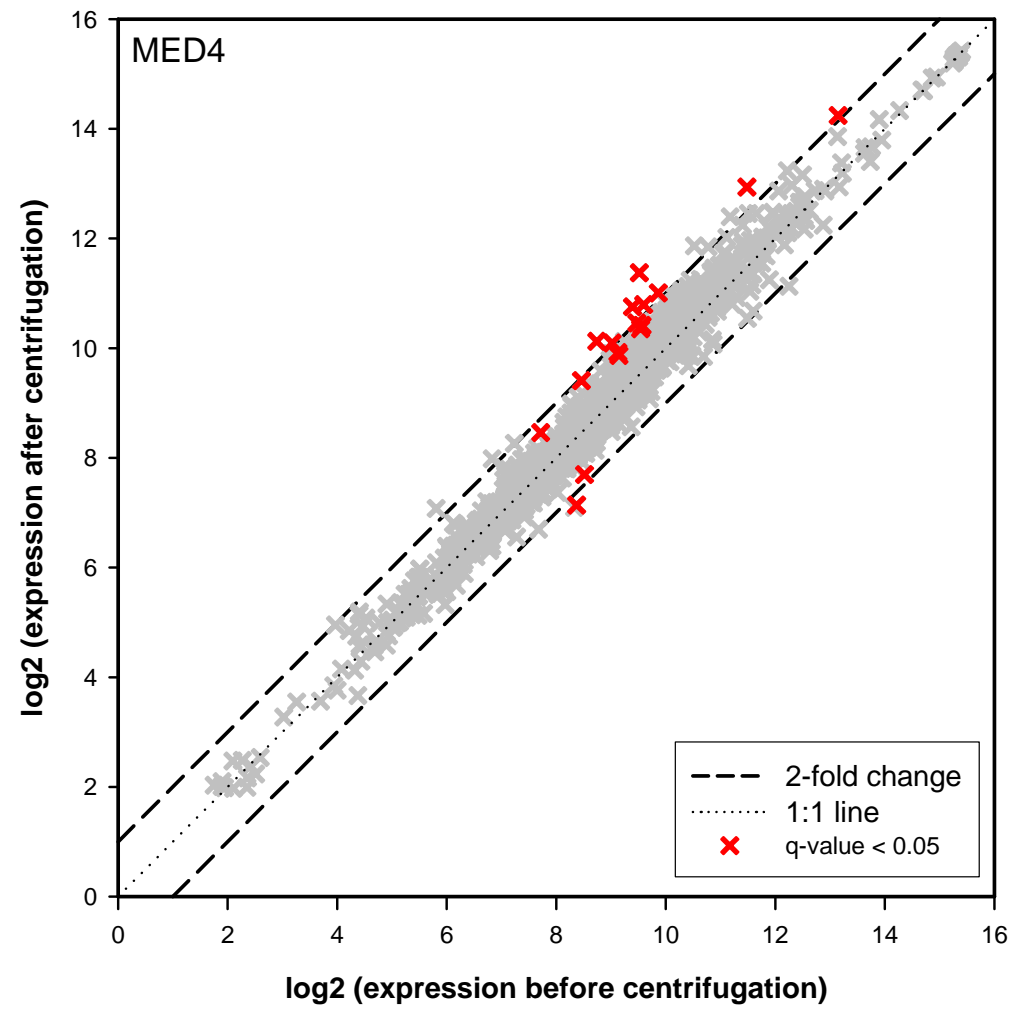

Figure 1: The effect of centrifugation on MED4 gene expression. Wholegenome expression before centrifugation (x-axis) is compared to whole-genome expression after centrifugation (y-axis) for duplicate cultures in each condition. Genes represented in red have q-value less than 0.05 . Genes with fold change greater than 2 and q-value less than 0.05 are defined as differentially expressed and are discussed in the text. 
APPENDiX E

Phylogeny of Prochlorococcus in an oxygen minimum zone off the coast of Mexico

\author{
Anne Thompson ${ }^{1}$ \\ Adam Martiny $^{2}$ \\ James Moffett ${ }^{3}$ \\ Allison $\mathrm{Coe}^{4}$ \\ Sallie W. Chisholm ${ }^{4,5}$
}

\footnotetext{
${ }^{1}$ MIT-WHOI Joint Program in Biological Oceanography

${ }^{2}$ University of California, Irvine - Departments of Earth System Science and Ecology and Evolutionary Biology

${ }^{3}$ University of Southern California - Department of Biological Sciences

${ }^{4}$ MIT Department of Civil and Environmental Engineering

${ }^{5}$ MIT Department of Biology
} 


\section{Introduction and Motivation}

Oxygen minimum zones (OMZ) are a widespread feature of upwelling systems in the oceans worldwide. Generally, OMZs are characterized by low oxygen $(<1 \mu \mathrm{m})$, low light $(0.1-2 \%$ surface irradiance), low temperature, elevated dissolved iron concentrations ( $>1 \mathrm{nM}$ compared to $0.3 \pm 0.1 \mathrm{nM}$ in the secondary chlorophyll maximum), and elevated $\mathrm{Fe}(\mathrm{II})$ concentrations $(0.2 \mathrm{nM})$ (Goericke et al, 2000; Johnson et al, 1999; Moffett et al, 2007). On multiple occasions, in the Eastern Tropical North Pacific (ETNP) and the Arabian Sea, dense and nearly monoalgal populations of Prochlorococcus have been observed within the OMZ (Goericke et al, 2000; Johnson et al, 1999). These populations actively fix carbon, contain high levels of divinyl chlorophyll a and b (characteristic of Prochlorococcus), and exhibit elevated chlb $\mathrm{b}_{2} / \mathrm{a}_{2} \mathrm{ratios}$, indicative of low light acclimatization (Goericke et al, 2000; Johnson et al, 1999). Here, cyanobacterial 16S/23S ITS sequences from an oxygen minimum zone off the coast of Mexico are cloned and sequenced then compared to clones from overlying oxygenated surface water and cultured isolates.

\section{Methods}

Sample collection and DNA extraction - Samples were collected off the coast of Mexico on November 19th of 2003 (Station 16 , CTD cast $19,18.04^{\circ} \mathrm{N}, 106.7^{\circ} \mathrm{W}$ ) on board the R/V New Horizon (MEX0311). Niskin bottles on a 13-bottle rosette were used to collect water from the chlorophyll maximum at 60 meters and the secondary chlorophyll maximum at 130 meters. Temperature, chlorophyll $a$ fluorescence, and dissolved oxygen concentrations were measured via the CTD at the same time of sample collection (Figure 1).

DNA samples were collected by filtering $100 \mathrm{ml}$ of sample water onto $25 \mathrm{~mm} 0.2 \mu \mathrm{m}$ pore size polycarbonate filters, rinsed with $3 \mathrm{ml}$ of preservation solution $(10 \mathrm{mM}$ Tris $\mathrm{pH} 8.0,100 \mathrm{mM}$ EDTA, 0.5M NaCl), then frozen in liquid nitrogen as per (Zinser et al, 2006). Samples were transported to the lab in a liquid nitrogen dry shipper and stored at $-80^{\circ} \mathrm{C}$ until processing. DNA was extracted from samples following the Prochlorococcus PCR template protocol outlined in (Zinser et al, 2006)

16S/23S ITS region cloning and sequencing - The 16S/23S ITS region was amplified using universal cyanobacterial primers. A reconditioning PCR protocol was followed where cycling conditions were: for 20 cycles followed with five replicate 1:10 dilutions of the PCR products 
into fresh reagents up to $50 \mu 1$ per sample (Thompson et al, 2002 ). Three additional PCR cycles were performed using the diluted template at the under the same cycle conditions as the first round of PCR. Reconditioned PCR products were then pooled for each sample and cloned into one-shot competent Escherichia coli (TOP10 cells) using the TOPO TA kit (Invitrogen)

following the manufacturer's protocol. Cloned products were sequenced commercially by the Marine Biological Laboratory (Woods Hole, MA). The Prochlorococcus ARB database of ITS sequences was used to align the sequences and produce phylogenetic trees.

\section{Results}

Clones from within the oxygen minimum zone were most similar to the low-light adapted Prochlorococcus isolates of MIT9313 and MIT9303 (Figure 1). Furthermore, clones from within the OMZ were phylogenetically distinct from cyanobacterial clones in the oxygenated water at $60 \mathrm{~m}$ depth that both formed a separate group of sequences and clustered with the two known lowlight adapted Prochlorococcus ecotypes MIT9313 and MIT9303. Further work is needed to understand if all Prochlorococcus within oxygen minimum zones are phylogenetically similar or if the distinct population observed here is unique to the $\mathrm{OMZ}$ that we sampled. 


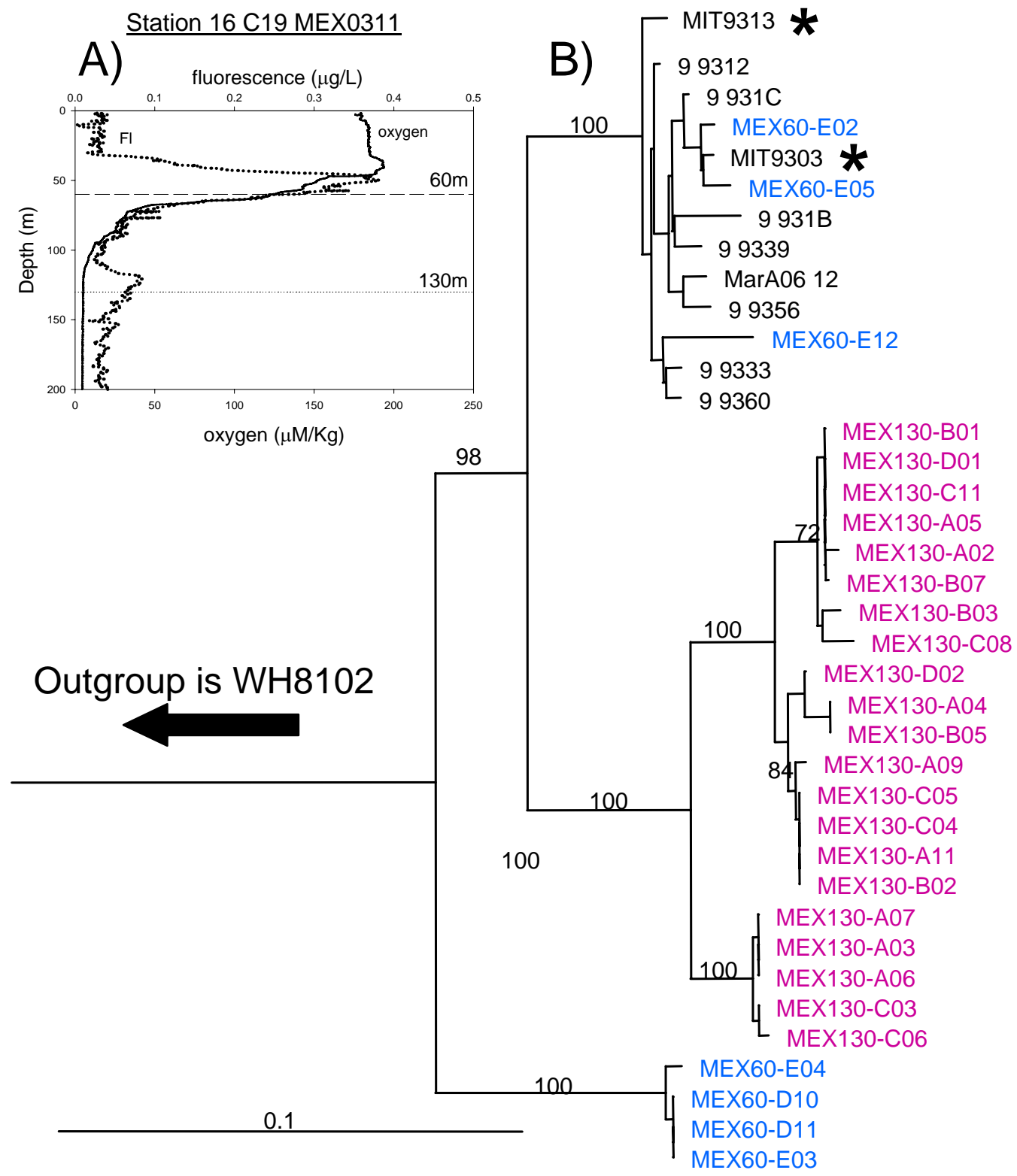

Figure 1: Phylogeny of Prochlorococcus from an oxygen minimum zone (OMZ) off the coast of Mexico in 2003. (A) Hydrographic data collected by CTD showing a peak in fluorescence within the OMZ. (B) Sequences from the OMZ, $130 \mathrm{~m}$, are in pink and are most closely-related to the low-light adapted ecotypes MIT9313 and MIT930 (black with asterisk). Sequences in blue are from $60 \mathrm{~m}$. Note that sequence identified as "9 9312" is not the same as MIT9312. 


\section{APPENDIX F}

Scanning electron microscopy of a non-axenic culture of Prochlorococcus, MIT9215 


\section{Introduction and Motivation}

The motivation for this project was to collect scanning electron microscopy images of Prochlorococcus for general use in the lab. In addition, an image of MIT9215 was required to accompany the genome sequence of this Prochlorococcus strain which is published by the Joint Genome Institute (JGI) on-line (http://genome.jgipsf.org/finished_microbes/prom5/prom5.home.html).

\section{Methods}

Claire Ting (Williams University) was able to get clear images of the exterior/surface of Prochlorococcus using scanning electron microscopy (SEM). SEM sample preparation was carried out as described in the unpublished document, "The fine structure of the Marine Photosynthetic Prokaryote, Prochlorococcus" with a slight modification that extended the times in the alcohol dehydration step from 15 minutes in each 50\%, 75\%, 95\%, and 100\% to 30 minutes in each step as recommended by Erika Batchelder of the Keck Microscopy Facility at the Whitehead Institute.

The services of the Whitehead Institute Keck Microscopy facility were used for consulting on fixation steps and for critical point drying and conductive metal sputter-coating. SEM images were taken at the Whitehead Institute by Erika Batchelder on their JEOL JSM-5600LV. This microscope allowed visualization of the cells but not at very high magnification or resolution.

Strain MIT9215, growing in PRO99 at a light intensity of $16 \mu \mathrm{Em}^{-2} \mathrm{~s}^{-2}$, was sampled for SEM in late log phase growth when cell densities were high. Cells were concentrated by centrifugation at $12,000 \mathrm{xg}$ for 10 minutes prior to fixation.

In order to obtain high resolution images, a JEOL JSM-6320FV Field-Emission Highresolution scanning electron microscope run by Yong Zhang at the Center for Materials Science at MIT (http://materials.mit.edu) was used to obtain the images displayed here. Using this machine, we were able to capture high resolution and high magnification images where even surface texture of the cells could be observed.

\section{Results}

A high resolution/high magnification image of Prochlorococcus MIT9215 (not axenic) was obtained using the JEOL JSM-6320FV. The image in Figure 1A is focused on two cells about to complete division. The high resolution and magnification allows us to begin to see some of the 
cell's surface texture. The culture used for this image was in stationary phase. Some cell debris is apparent in the images in the form of cells that appear deflated or burst. It is unclear if these cells existed in the culture or if their shape and texture is an artifact of sample preparation. The cells in Figure 1A are spherical and about $0.6 \mu \mathrm{m}$ in diameter. The shape of MIT9215 is comparable to MED4 which is also spherical and is expected based on their phylogenetic similarity. The cell size of MIT9215 is slightly larger than the size reported for MED4 by the same method but it is very possible that the difference may be attributed to different growth conditions or sample preparation (Ting et al, unpublished).

We obtained images of larger fields of cells that include heterotrophic contaminants on both the JEOL JSM-5600LV and JEOL JSM-6320FV. Figure 1B shows images from the JEOL JSM$5600 \mathrm{LV}$ with rod-like contaminants (black arrows) and very small (about $1 / 10^{\text {th }}$ the size of Prochlorococcus) spherical objects that often appear to be associated with the Prochlorococcus cells though not in all cases (white arrows). These small spherical objects are also visible in SEM images of axenic MED4 (Ting et al, unpublished). 

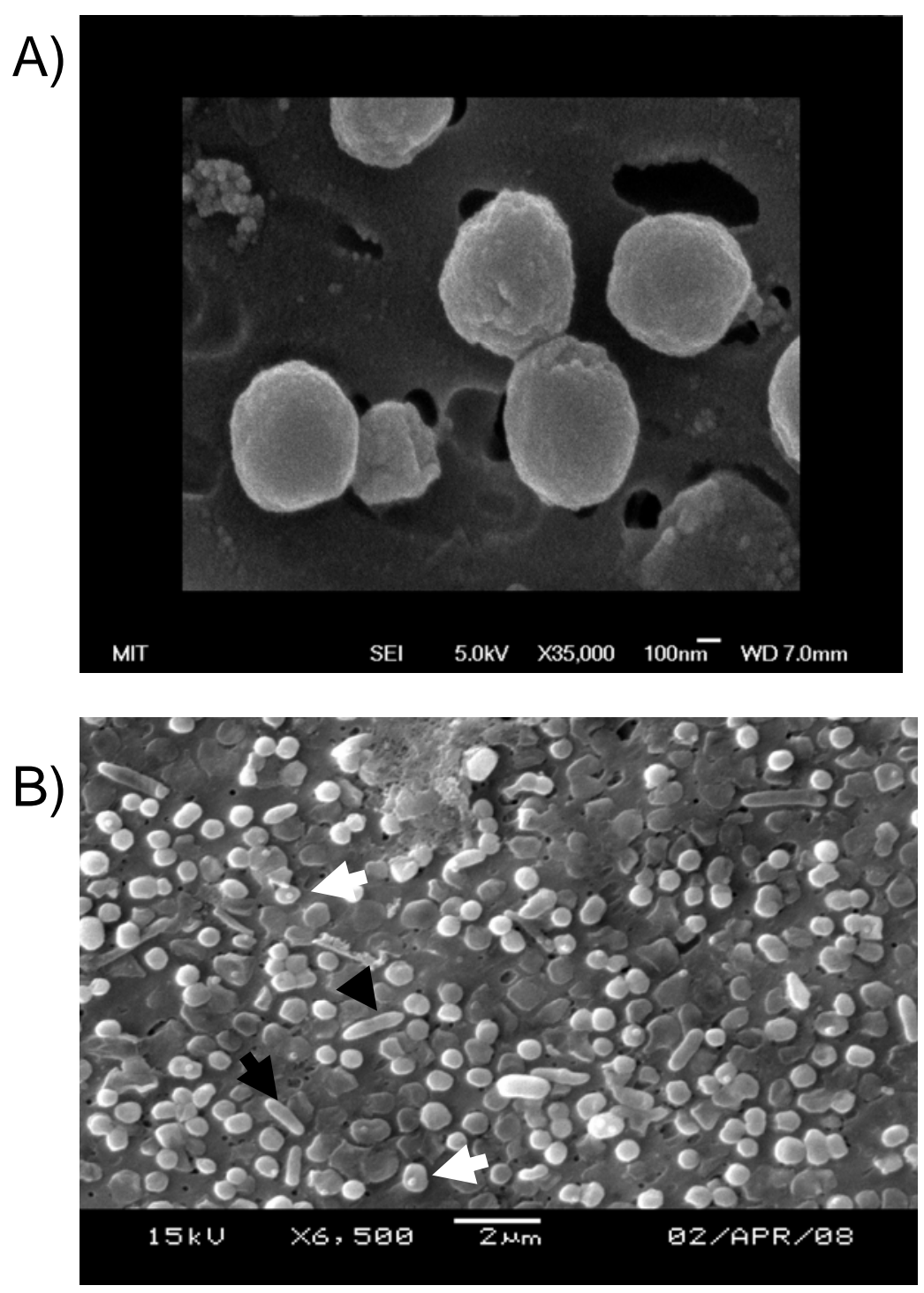

Figure 1: Scanning electron microscopy images of non-axenic MIT9215. (A) A high resolution/high magnification image of Prochlorococcus MIT9215 (not axenic) was obtained using the JEOL JSM-6320FV. (B) Images from the JEOL JSM-5600LV with rod-like contaminants (black arrows) and very small (about 1/10th the size of Prochlorococcus) spherical objects that often appear to be associated with the Prochlorococcus cells though not in all cases (white arrows). These small spherical objects are also visible in SEM images of axenic MED4 taken by Claire S. Ting (data unpublished). 


\title{
APPENDIX G
}

The abundance and distribution of Prochlorococcus and Synechococcus in the Eastern Equatorial Pacific Ocean

\author{
Anne Thompson ${ }^{1}$ \\ Mak A. Saito ${ }^{2}$ \\ James Moffett ${ }^{3}$ \\ Sallie W. Chisholm ${ }^{4}$
}

\footnotetext{
${ }^{1}$ MIT-WHOI Joint Program in Biological Oceanography

${ }^{2}$ Woods Hole Oceanographic Institution - Department of Marine Chemistry and Geochemistry

${ }^{3}$ University of Southern California - Department of Biological Sciences

${ }^{4}$ MIT Departments of Civil and Environmental Engineering and Biology
} 


\section{Introduction and Motivation}

The coast of Peru is known for its high productivity which is due to the upwelling of high concentrations of major nutrients and iron from the continental shelf. At odds with its reputation, parts of the Peru upwelling system can be characterized as high-nitrate low-chlorophyll (HNLC) regimes where low iron availability limits primary production (Bruland et al, 2005; Strickland et al, 1969; Thomas, 1972, 1979). These HNLC regimes of the Peru upwelling regime occur where the continental shelf is narrow as it is off the coast of southern Peru and up-welled water contains low nutrient concentrations. In October and November of 2005, during the season of maximum coastal upwelling, samples were collected in a series of on-shore to off-shore transects that aimed to describe the abundance and distribution of Prochlorococcus and Synechococcus in the Peru upwelling regime. Surface samples were also collected while in transit from the northern coast of Peru to Mexico to expand our understanding of the global distribution and abundance of Prochlorococcus and Synechococcus.

We also visited the Costa Rica Upwelling Dome, which is an area of elevated primary productivity formed by wind driven upwelling of nutrient rich water off the coast of Costa Rica. Once the feature reaches the open ocean, the upwelling is strongest during the summer and diminishes in the late fall (Hofmann et al, 1981). The dome is a unique open ocean feature as its picophytoplankton community is dominated by Synechococcus instead of Prochlorococcus presumably as a result of the elevated nutrient concentrations supplied to surface water during the upwelling period (Fiedler, 2002; Hofmann et al, 1981; Li et al, 1983; Saito et al, 2005). The aim of the research cruise was to further characterize the suite of trace metals that are delivered to the surface during upwelling and further characterize the trace metal chemistry and microbial community of this unusual open ocean feature.

\section{Methods}

Depth profile sample collection - Samples were collected off the coast of Peru during October and November of 2005 on board the R/V Knorr (KN182-9) and during July and August of 2005 on board the R/V Knorr (KN182-5). Samples were collected by several different methods including Niskin bottles on a 24 bottle rosette equipped with a CTD, 10L trace metal clean GoFlo bottles (General Oceanics) deployed individually on a Kevlar line, and 5L trace metal clean Niskin bottles on a 6 bottle mini-rosette and Kevlar line, and 2.5L Go-Flo bottles on a 6-bottle rosette (General Oceanics) on a Kevlar line. Single $1 \mathrm{ml}$ samples for flow cytometry were 
preserved with $5 \mu 125 \%$ glutaraldehyde then flash frozen in liquid nitrogen and stored at $-70^{\circ} \mathrm{C}$. Samples were transported in liquid nitrogen to the lab at the end of the cruise and stored at $-80^{\circ} \mathrm{C}$ until processing.

In-transit surface sample collection - Samples were collected on board the R/V Knorr during transit time between northern coastal Peru and Manzanillo, Mexico in November 2005. Water samples from the ship's flow through water system were collected in 1L volumes in a dark plastic bottle that had been soaked in $10 \%$ bleach for at least one hour prior to use. Sampling was always preceded by running the water for 5 minutes before sampling to clear contamination and carryover from the flow through system. From each $1 \mathrm{~L}$ sample, triplicate $100 \mathrm{ml}$ samples were filtered onto $25 \mathrm{~mm} 0.2 \mu \mathrm{m}$ pore size polycarbonate filters using a $10 \%$ bleach-treated multi-channel peristaltic pump system (Masterflex) and 25mm swinnex filter holders (Millipore). Each sample filter was then rinsed with $3 \mathrm{ml}$ preservation solution (10mM Tris $\mathrm{pH} 8.0,100 \mathrm{mM}$ EDTA, $0.5 \mathrm{M}$ $\mathrm{NaCl}$ ), and frozen in liquid nitrogen as per the protocol outlined in (Zinser et al, 2006). 1ml samples for flow cytometry were fixed with $5 \mu 125 \%$ glutaraldehyde (Tousmis), incubated in the dark for 10 minutes and frozen in liquid nitrogen and stored at $-70^{\circ} \mathrm{C}$. Samples were transported to the lab in liquid nitrogen dry shippers and stored at $-80^{\circ} \mathrm{C}$ until processing.

Flow cytometry - Flow cytometry samples were analyzed using an Influx flow cytometer (Cytopeia, Seattle, WA). Red fluorescence and forward angle light scatter were used to identify populations of Prochlorococcus and Synechococcus (Cavender-Bares et al, 1999). 


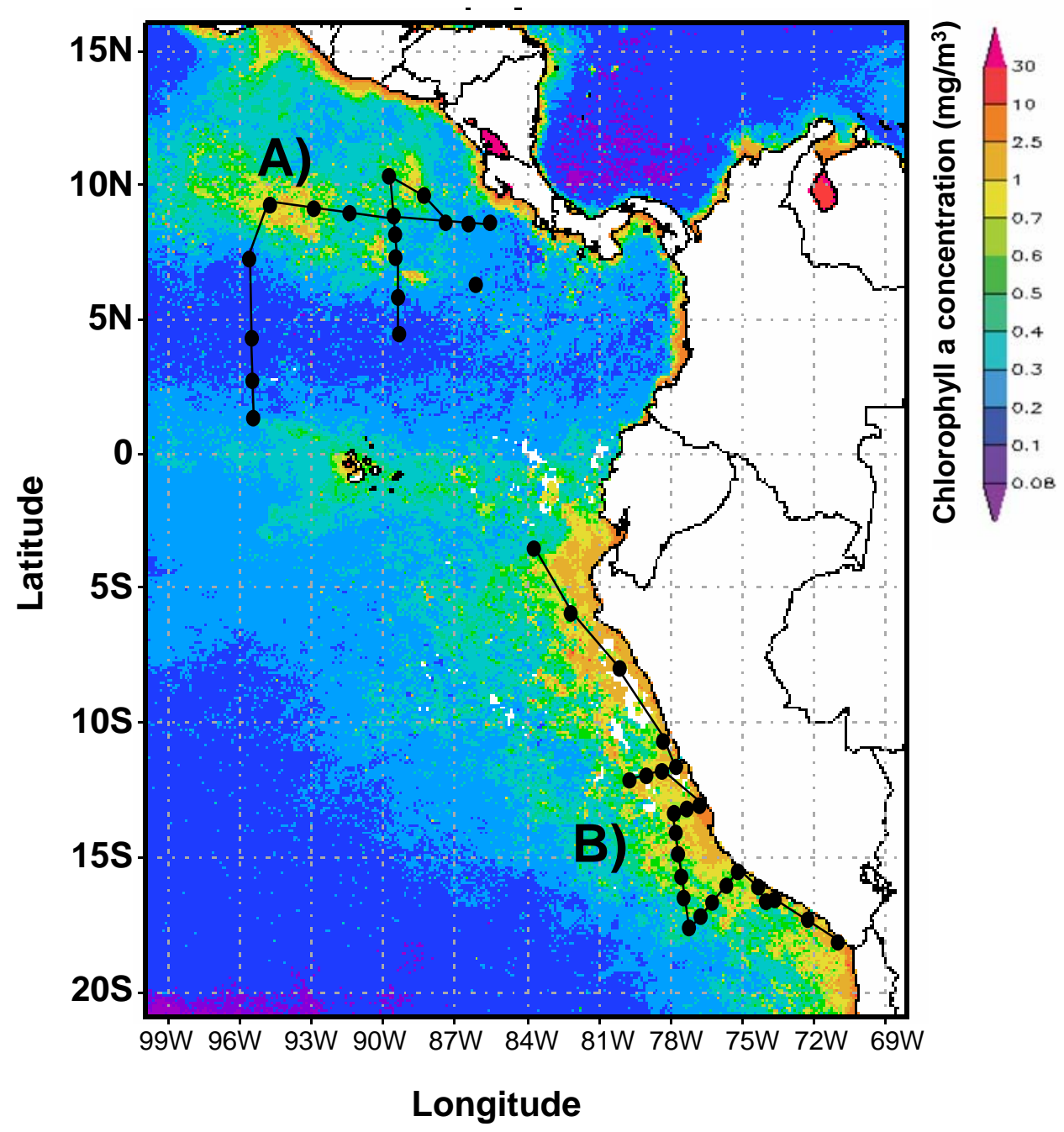

Figure 1: Cruise tracks of KN182-5 (July 2005) (A) and KN182-9 (October 2005) (B). Map displays chlorophyll concentrations and was generated by NASA's Giovanni (giovanni.gsfc.nasa.gov) at 9km-resolution for the period of July to October 2005. 


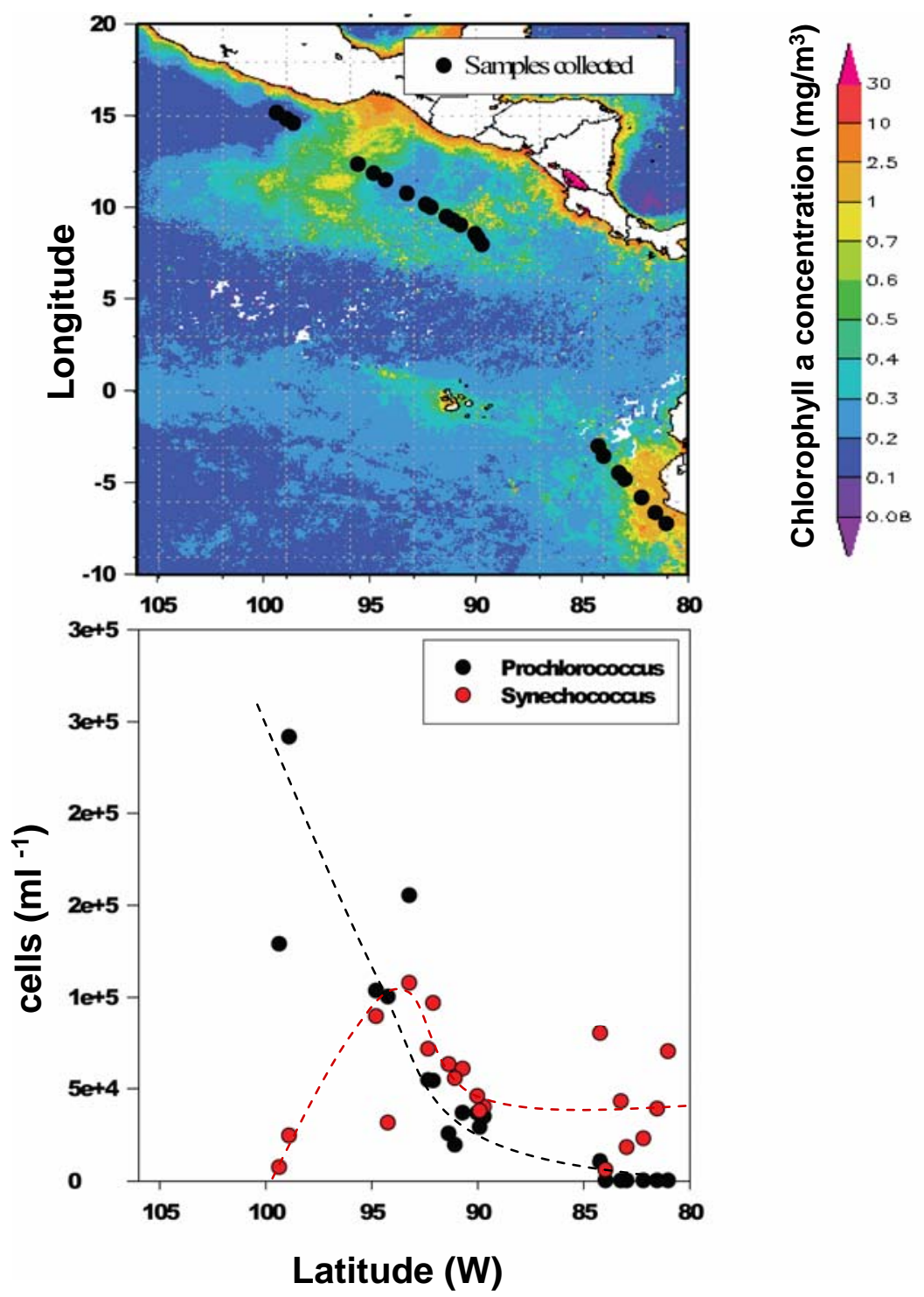

Figure 2: Prochlorococcus and Synechococcus abundances in surface transect through Eastern Equatorial Pacific on cruise KN182-9. Top panel generated by NASA's Giovanni (giovanni.gsfc.nasa.gov) and displays 9km-resolution chlorophyll a concentrations compiled for the months of October and November 2005. 

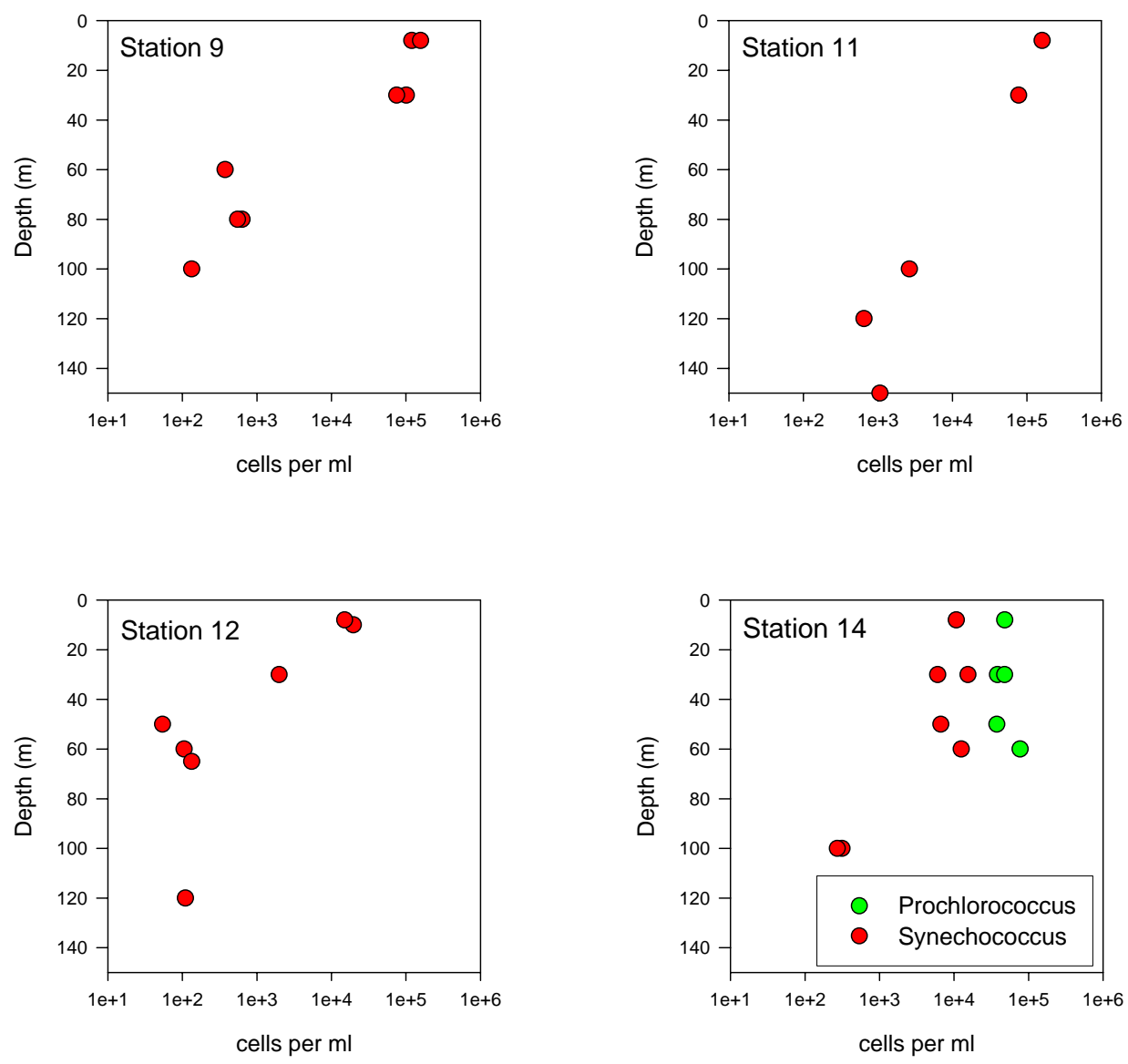

Figure 3: Abundances of Prochlorococcus and Synechococcus in the Peru Upwelling Region on cruise KN182-9. 

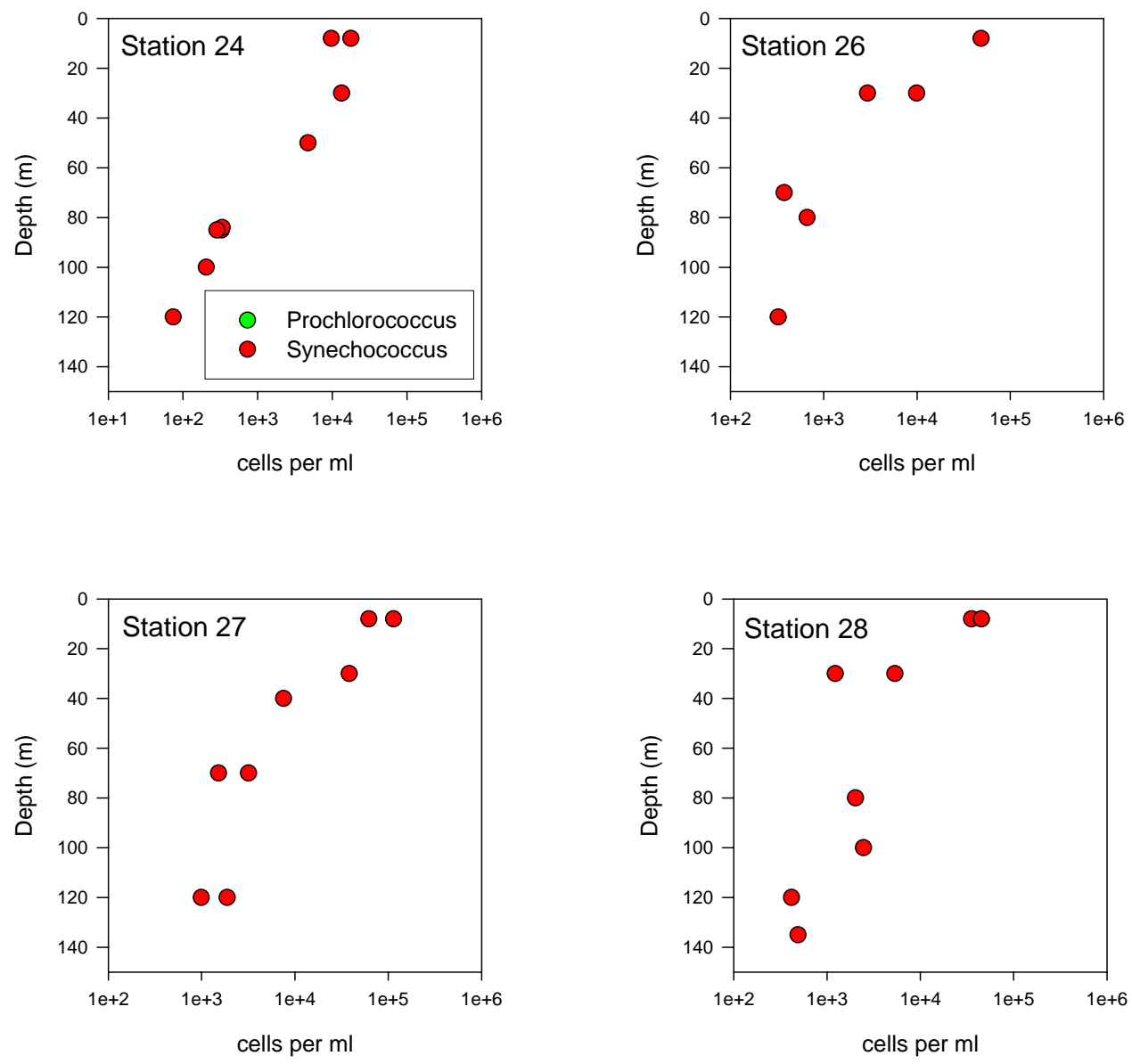

Figure 3, continued: Abundances of Prochlorococcus and Synechococcus in the Peru Upwelling Region on cruise KN182-9. 

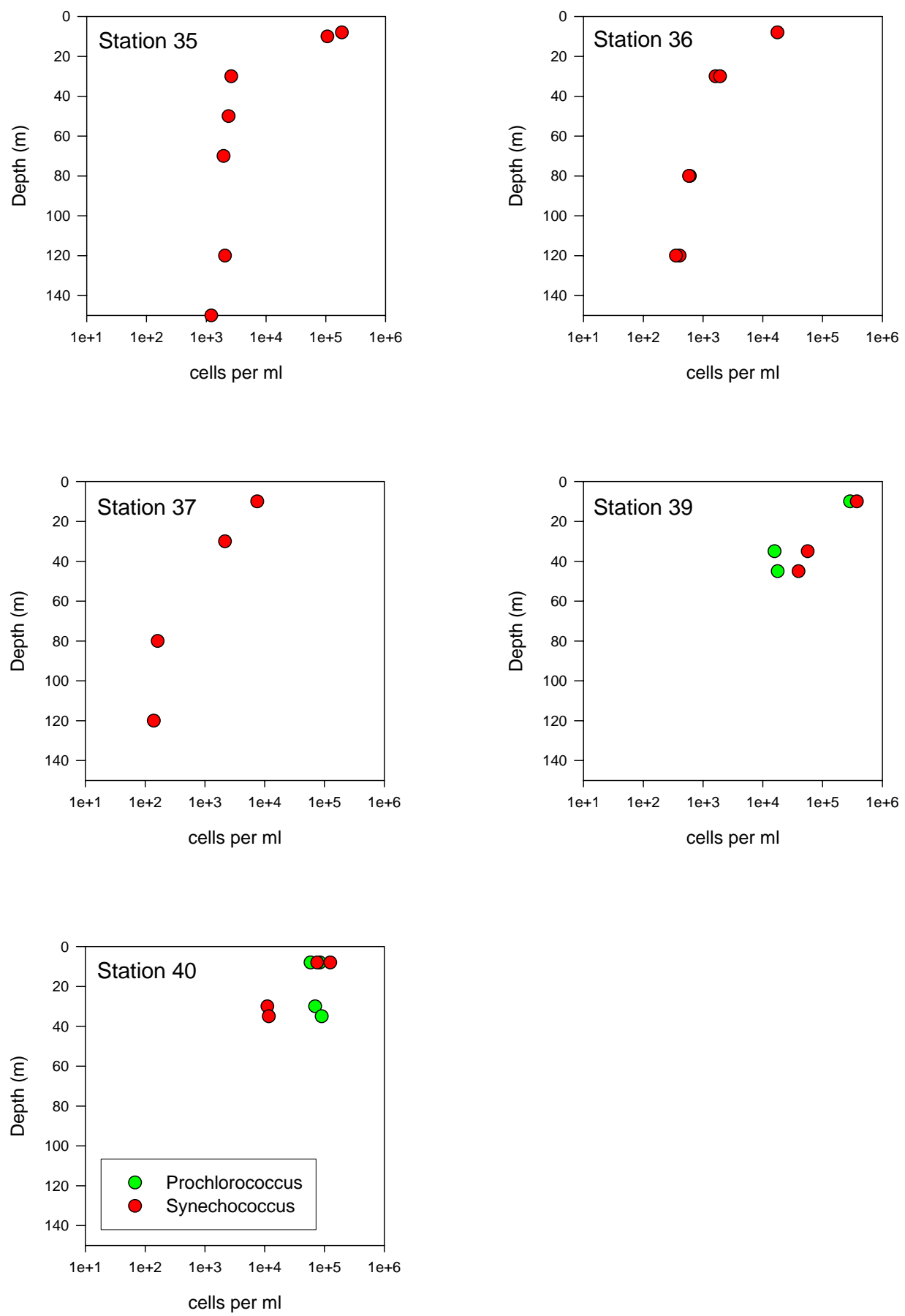

Figure 3, continued: Abundances of Prochlorococcus and Synechococcus in the Peru Upwelling Region on cruise KN182-9. 

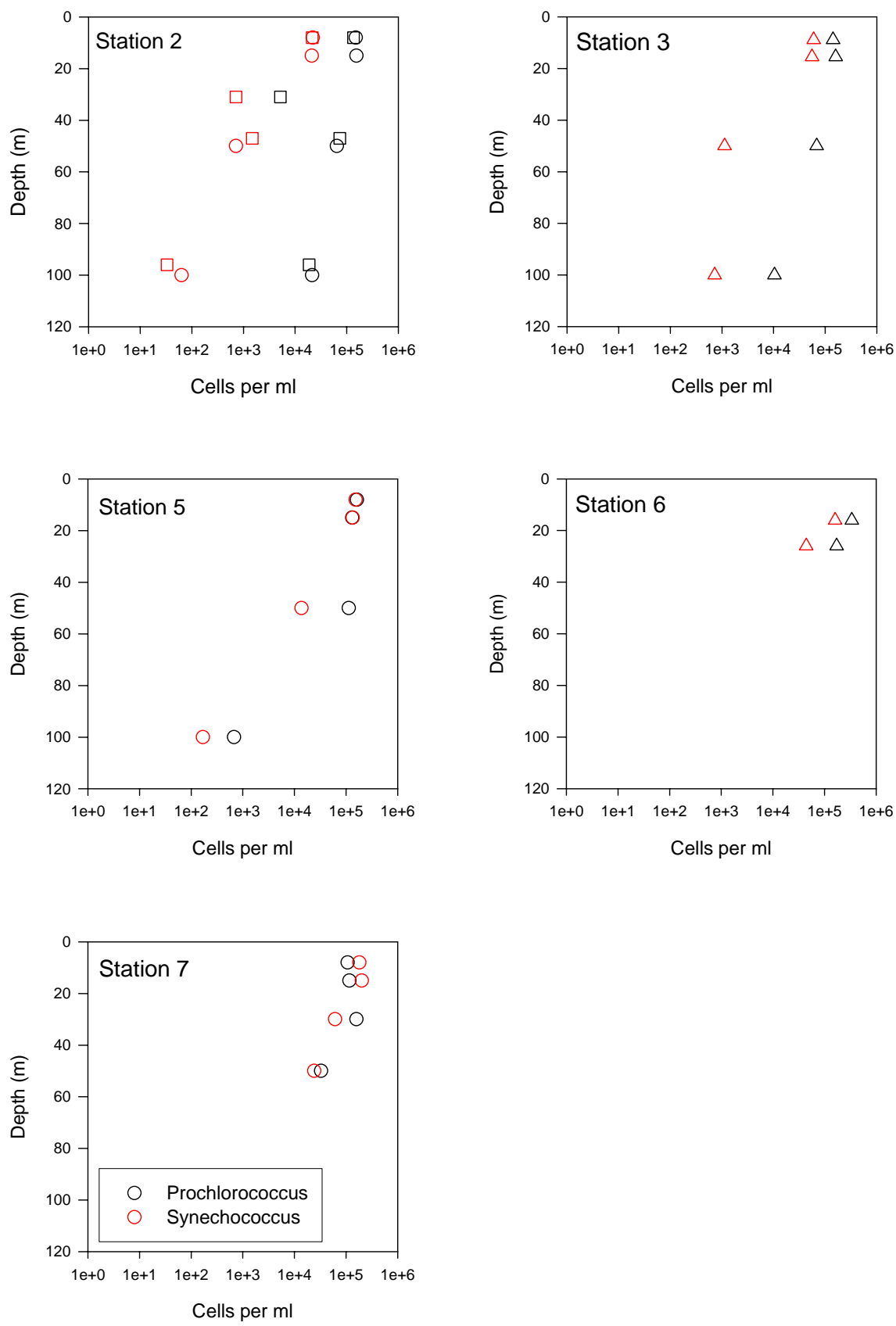

Figure 4: Abundances of Prochlorococcus and Synechococcus in the vicinity of the Costa Rica Upwelling Dome on cruise KN182-5. Circles represent samples collected by 10L Go-Flo, triangles represent samples collected by CTD, squares represent samples collected by the mini trace-metal clean rosette. 

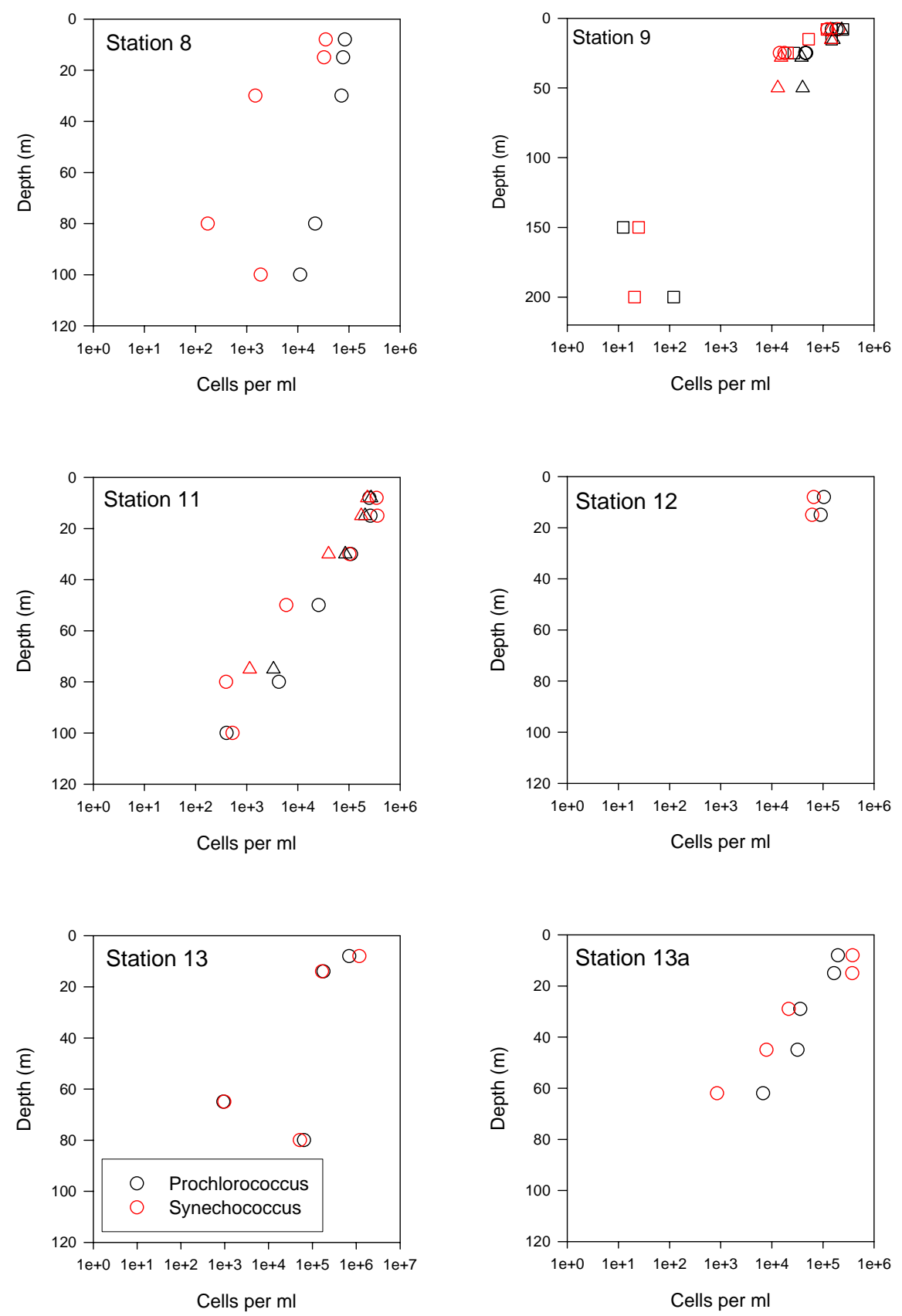

Figure 4, continued: Abundances of Prochlorococcus and Synechococcus in the vicinity of the Costa Rica Upwelling Dome on cruise KN182-5. Circles represent samples collected by 10L Go-Flo, triangles represent samples collected by CTD, squares represent samples collected by the mini trace-metal clean rosette. 

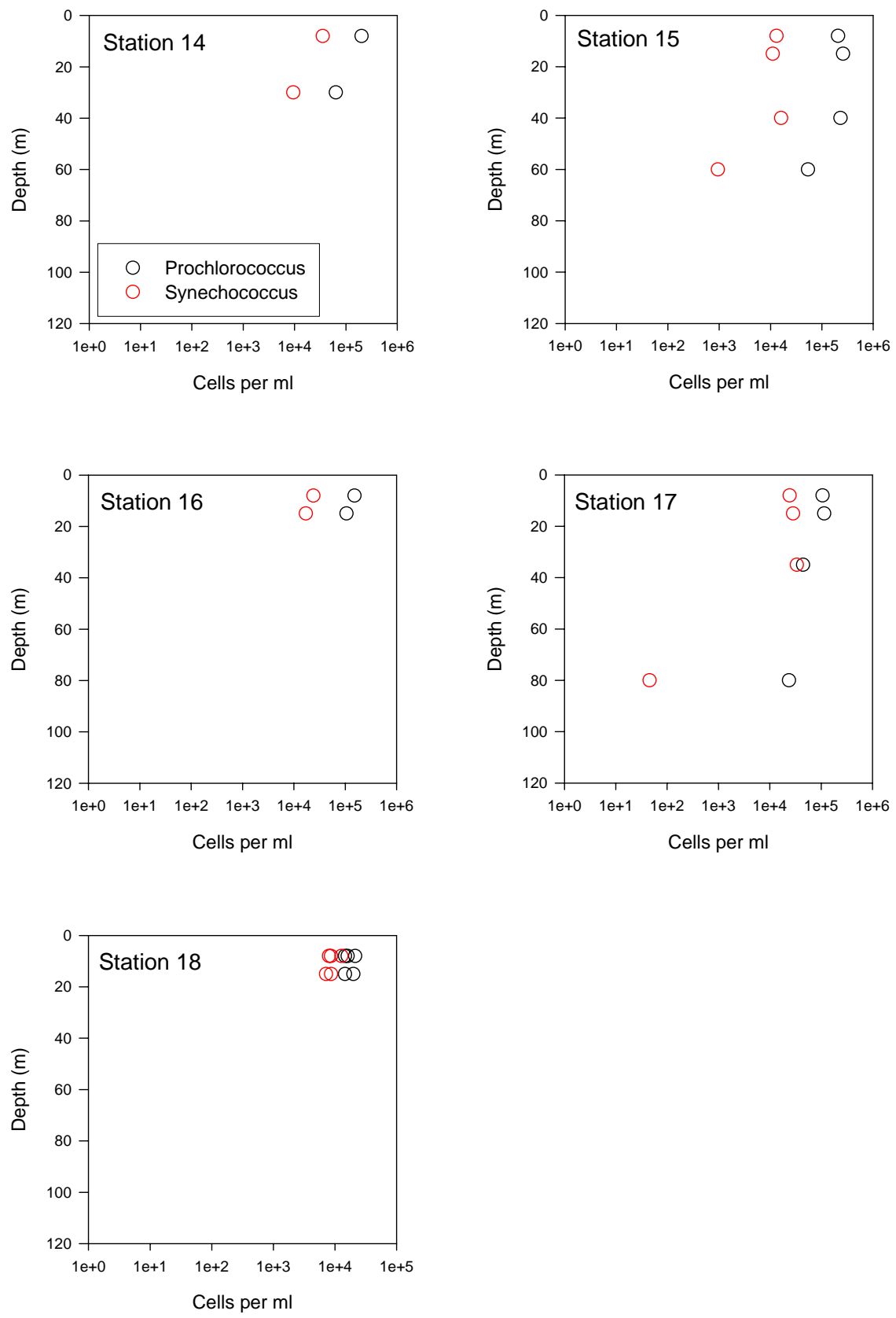

Figure 4, continued: Abundances of Prochlorococcus and Synechococcus in the vicinity of the Costa Rica Upwelling Dome on cruise KN182-5. Circles represent samples collected by 10L Go-Flo, triangles represent samples collected by CTD, squares represent samples collected by the mini trace-metal clean rosette. 

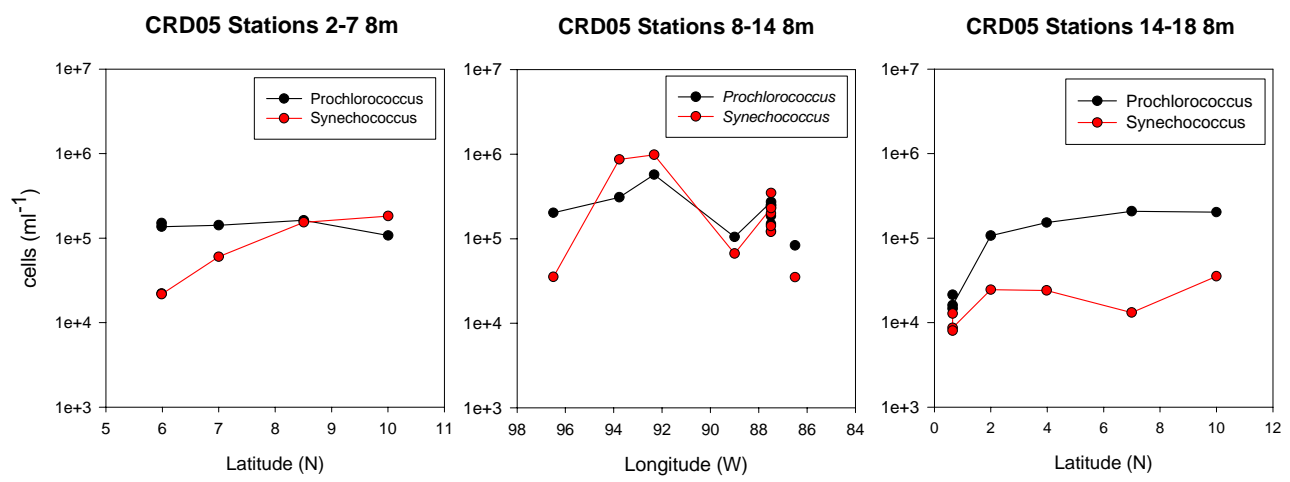

Figure 5: Surface abundances of Prochlorococcus and Synechococcus across the Costa Rica Upwelling Dome on cruise KN182-5. 


\title{
APPENDIX H
}

Prochlorococcus and Synechococcus response to iron and cobalt enrichment in the Costa Rica Upwelling Dome in July 2005

\author{
Anne Thompson ${ }^{1}$ \\ Mak A. Saito ${ }^{2}$ \\ Sallie W. Chisholm ${ }^{3}$
}

\footnotetext{
${ }^{1}$ MIT-WHOI Joint Program in Biological Oceanography

${ }^{2}$ Woods Hole Oceanographic Institution - Department of Marine Chemistry and Geochemistry

${ }^{3}$ MIT Departments of Civil and Environmental Engineering and Biology
} 

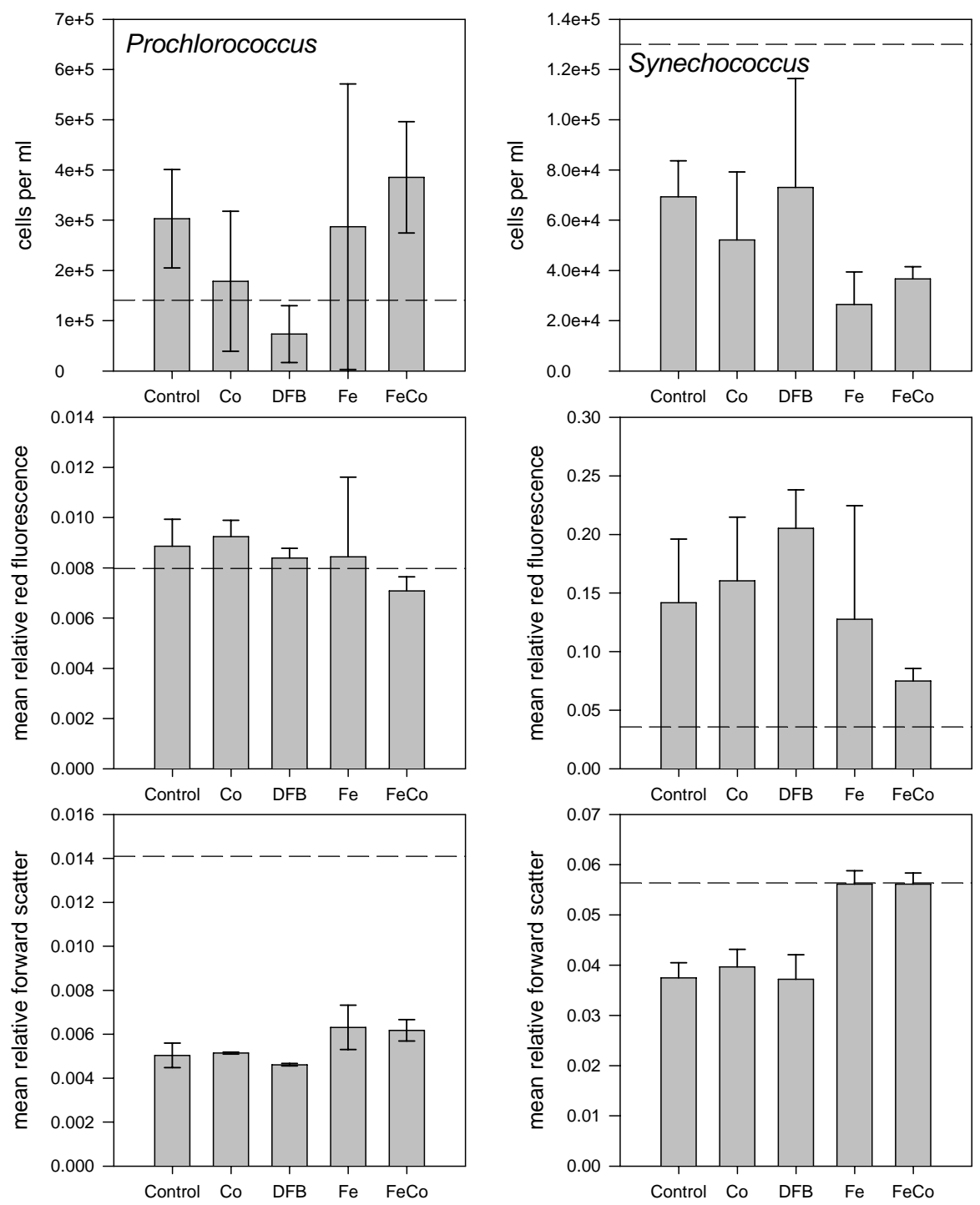

Figure 1: Bottle incubation experiments at Station 5 on cruise KN182-5. Triplicate 1.2L volumes of seawater collected under trace-metal clean conditions and were amended with 20nM DFB, $1 \mathrm{nM} \mathrm{FeSO}_{4}, 0.5 \mathrm{nM} \mathrm{CoCl}_{2}$, or both $\mathrm{Fe}$ and $\mathrm{Co}$. The control treatment was un-amended. Incubations lasted about 72 hours in on-deck incubators cooled with surface seawater. Flow cytometry was used to measure cell concentration, red fluorescence per cell, and forward angle light scatter as described in Chapter 4 (Methods). Dashed line represents the seawater sample at time zero, prior to additions. 

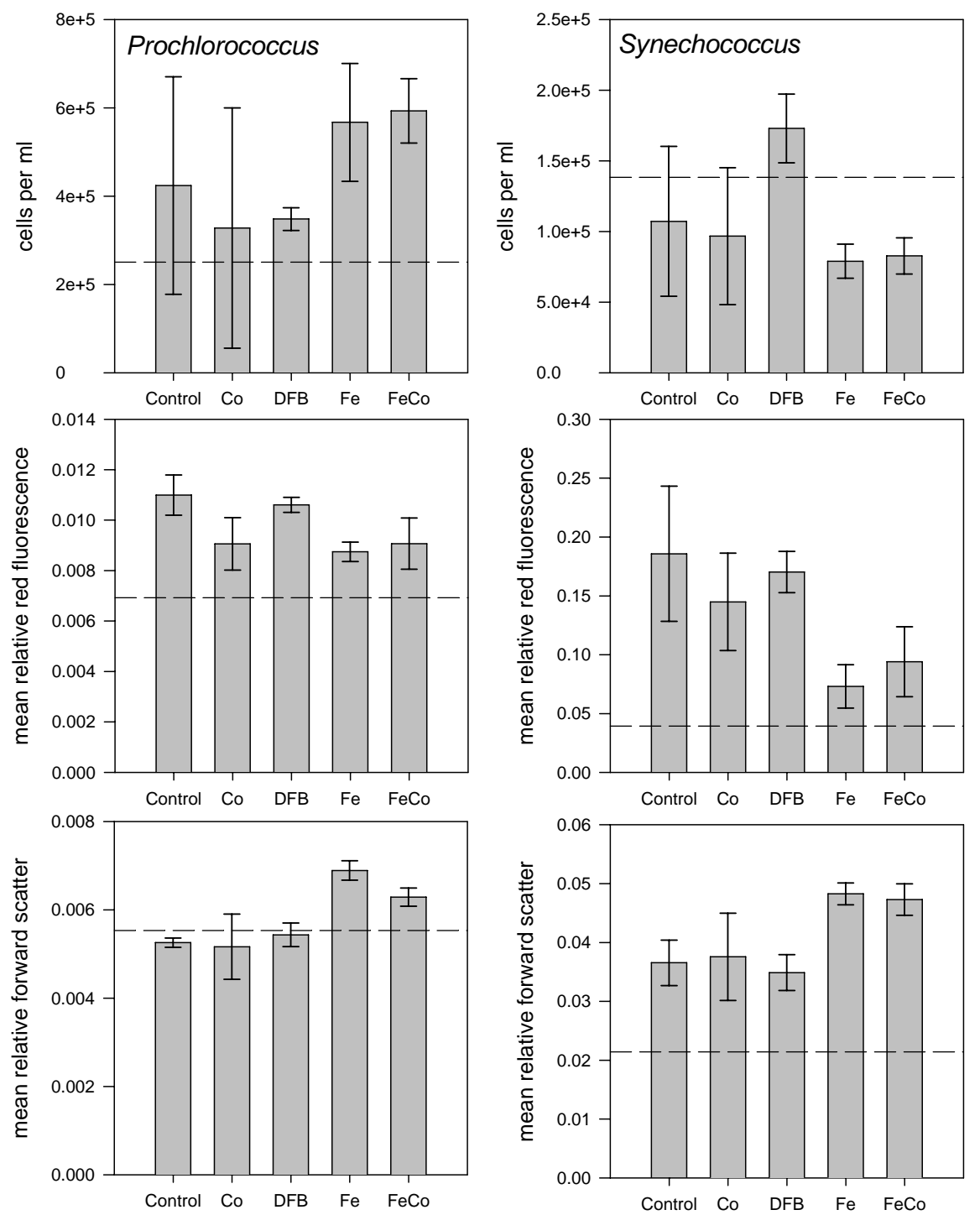

Figure 2: Bottle incubation experiments at Station 11 on cruise KN182-5. Incubation conditions are identical to those described in Figure 1. 

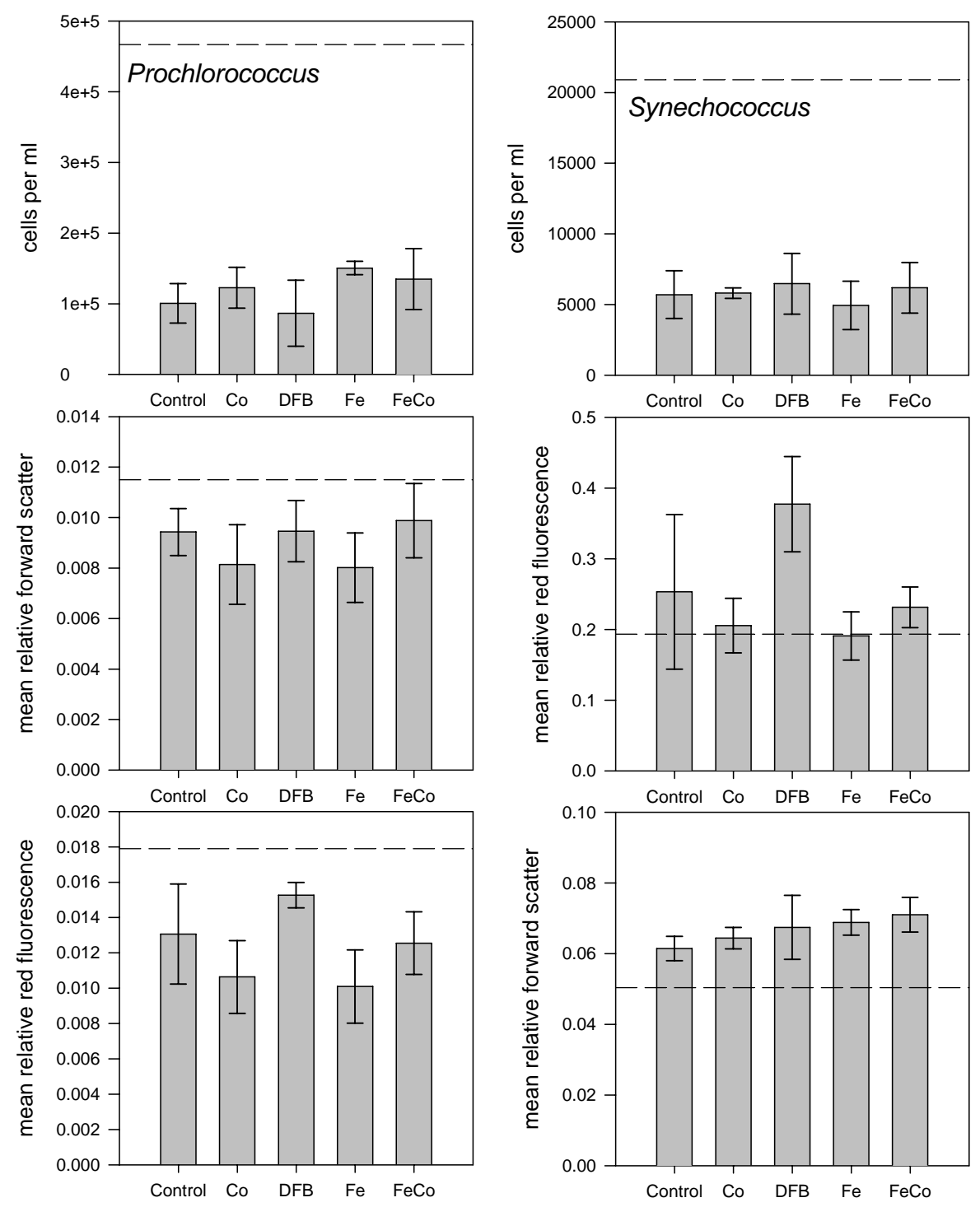

Figure 3: Bottle incubation experiments at Station 15 on cruise KN182-5. Incubation conditions are identical to those described in Figure 1. 


\section{Appendices References}

Bruland KW, Rue EL, Smith GJ, DiTullio GR (2005) Iron, macronutrients and diatom blooms in the Peru puwelling regime: brown and blue waters of Peru. Marine Chemistry 93: 81-103.

Cavender-Bares KK, Mann EL, Chisholm SW, Ondrusek ME, Bidigare RR (1999) Differential response of equatorial Pacific phytoplankton to iron fertilization. Limnology and Oceanography 44: 237-246.

Fiedler PC (2002) The annual cycle and biological effects of the Costa Rica dome. Deep Sea Research Part I: Oceanographic Research Papers 49: 321-338.

Goericke R, Olson RJ, Shalapyonok A (2000) A novel niche for Prochlorococcus sp. in low-light suboxic environments in the Arabian Sea and the Eastern Tropical North Pacific. Deep Sea Research Part I: Oceanographic Research Papers 47: 1183-1205.

Hofmann EE, Busalacchi AJ, O'Brien JJ (1981) Wind generation of the Costa Rica dome. Science 214: 552-554.

Johnson Z, Landry ML, Bidigare RR, Brown SL, Campbell L, Gunderson J, Marra J, Trees C (1999) Energetics and growth kinetics of a deep Prochlorococcus spp. population in the Arabian Sea. Deep Sea Research Part II: Topical Studies in Oceanography 46: 1719-1743.

Li WKW, Subba Rao DV, Harrison WG, Smith JC, Cullen J, Irwin B, Platt T (1983) Autotrophic picoplankton in the tropical ocean. Science 219: 292-295.

Martiny AC, Coleman ML, Chisholm SW (2006) Phosphate acquisition genes in Prochlorococcus ecotypes: Evidence for genome-wide adaptation. Proceedings of the National Academy of Sciences 103: 12552-12557.

Moffett JW, Goepfert TJ, Naqvi SWA (2007) Reduced iron associated with secondary nitrite maxima in the Arabian Sea. Deep Sea Research Part I: Oceanographic Research Papers 54: 1341-1349.

Saito MA, Rocap G, Moffett JW (2005) Production of cobalt binding ligands in a Synechococcus feature at the Costa Rica upwelling dome. Limnology and Oceanography 50: 279-290.

Strickland JDH, Eppley RW, deMendiola BR (1969) Phytoplankton populations, nutrients and photosynthesis in Peruvian coastal waters. Biblioteca, Instituto Del Mar Del Peru 2: 4-45.

Thomas WH (1972) Nutrient inversions in the southeastern tropical Pacific Ocean. Fisheries Bulletin 70: 929-932.

Thomas WH (1979) Anomalous nutrient-chlorophyll interrelationships in the offshore eastern tropical Pacific Ocean. Journal of Marine Research 37: 327-335.

Thompson JA, Marcelino LA, Polz MF (2002) Heteroduplexes in mixed-template amplifications: formation, consequence and elimination by 'reconditioning PCR'. Nucleic Acids Res 30: 20832088. .

Ting CS, Chisholm SW, King J (unpublished) The Fine Structure of the Marine Photosynthetic Prokaryote, Prochlorococcus.

Tolonen AC, Aach J, Lindell D, Johnson ZI, Rector T, Steen R, Church GM, Chisholm SW (2006) Global gene expression of Prochlorococcus ecotypes in response to changes in nitrogen availability. Mol Syst Biol 2.

Zinser ER, Coe A, Johnson ZI, Martiny AC, Fuller NJ, Scanlan DJ, Chisholm SW (2006) Prochlorococcus Ecotype Abundances in the North Atlantic Ocean As Revealed by an Improved Quantitative PCR Method. Appl Environ Microbiol 72: 723-732. 